\title{
Planejamento Probabilístico com Becos sem Saída
}

\author{
Thiago Dias Simão \\ DissERTAÇÃO APRESENTADA \\ $\mathrm{AO}$ \\ Instituto de MatemáticA e EstatísticA \\ DA \\ Universidade DE SÃo PAUlo \\ PARA \\ OBTENÇÃO DO TÍTULO \\ $\mathrm{DE}$ \\ Mestre EM CiÊnCIAS \\ Programa: Ciência da Computação \\ Orientador: Prof ${ }^{a}$. Dr ${ }^{a}$. Leliane Nunes de Barros
}

Durante o desenvolvimento deste trabalho o autor recebeu auxílio financeiro da CAPES

São Paulo, Fevereiro de 2017 


\section{Planejamento Probabilístico com Becos sem Saída}

Esta é a versão original da dissertação elaborada pelo candidato (Thiago Dias Simão), tal como submetida à Comissão Julgadora. 


\section{Planejamento Probabilístico com Becos sem Saída}

Esta versão da dissertação contém as correções e alterações sugeridas pela Comissão Julgadora durante a defesa da versão original do trabalho, realizada em 06/03/2017. Uma cópia da versão original está disponível no Instituto de Matemática e Estatística da Universidade de São Paulo.

Comissão Julgadora:

- Prof $^{\mathrm{a}}$. Dr ${ }^{\mathrm{a}}$. Leliane Nunes de Barros (orientadora) - IME-USP

- Prof. Dr. Denis Deratani Mauá - IME-USP

- Prof. Dr. Silvio do Lago Pereira - FATEC 


\section{Agradecimentos}

Aos meu pais, Eliza e Acácio, pelo apoio e compreensão incondicionais.

À minha tia, Ângela, que me acolheu durante essa fase e sempre me incentivou a buscar meus sonhos.

À minha namorada, Mariane, por todo apoio e carinho.

À minha orientadora, professora Leliane, pelos ensinamentos e as inúmeras contribuições feitas para este trabalho.

Aos professores Silvio e Denis, pelas sugestões feitas.

Aos amigos do LIAMF, pelos incontáveis bons momentos.

Aos familiares e amigos que torceram por mim.

À CAPES, pela concessão da bolsa. 


\section{Resumo}

SIMÃO, T. D. Planejamento Probabilístico com Becos sem Saída. 2017. Dissertação (Mestrado) - Instituto de Matemática e Estatística, Universidade de São Paulo, São Paulo, 2017.

Planejamento probabilístico lida com a tomada de decisão sequencial em ambientes estocásticos e geralmente é modelado por um Processo de Decisão Markoviano (Markovian Decision Process $M D P)$. Um MDP modela a interação entre um agente e o seu ambiente: em cada estágio, o agente decide executar uma ação, com efeitos probabilísticos e um certo custo, que irá produzir um estado futuro. O objetivo do agente MDP é minimizar o custo esperado ao longo de uma sequência de escolhas de ação. O número de estágios que o agente atua no ambiente é chamado de horizonte, o qual pode ser finito, infinito ou indefinido. Um exemplo de MDP com horizonte indefinido é o Stochastic Shortest Path MDP (SSP MDP), que estende a definição de MDP adicionando um conjunto de estados meta (o agente para de agir ao alcançar um estado meta). Num SSP MDP é feita a suposição de que é sempre possível alcançar um estado meta a partir de qualquer estado do mundo. No entanto, essa é uma suposição muito forte e que não pode ser garantida em aplicações práticas. Estados a partir dos quais é impossível atingir a meta são chamados de becos-sem-saída. Um beco-sem-saída pode ser evitável ou inevitável (se nenhuma política leva do estado inicial para a meta com probabilidade um). Em trabalhos recentes foram propostas extensões para SSP MDP que permitem a existência de diferentes tipos de beco-sem-saída, bem como algoritmos para resolvê-los. No entanto, a detecção de becos-sem-saída é feita utilizando: (i) heurísticas que podem falhar para becos-sem-saída implícitos ou (ii) métodos mais confiáveis, mas que demandam alto custo computacional. Neste projeto fazemos uma caracterização formal de modelos de planejamento probabilístico com becos-sem-saída. Além disso, propomos uma nova técnica para detecção de becos-sem-saída baseada nessa caracterização e adaptamos algoritmos de planejamento probabilístico para utilizarem esse novo método de deteç̧ão. Os resultados empíricos mostram que o método proposto é capaz de detectar todos os becos-sem-saída de um dado conjunto de estados e, quando usado com planejadores probabilísticos, pode tornar esses planejadores mais eficientes em domínios com becos-sem-saída difíceis de serem detectados.

Palavras-chave: Planejamento Probabilístico, MDP, SSP MDP, Becos-sem-saída. 


\section{Abstract}

SIMÃO, T. D. Probabilistic planning with dead-ends. 2017. Dissertation (MSc.) - Instituto de Matemática e Estatística, Universidade de São Paulo, São Paulo, 2017.

Probabilistic planning deals with sequential decision making in stochastic environments and is modeled by a Markovian Decision Process (MDP). An MDP models the interaction between an agent and its environment: at each stage, the agent decides to execute an action, with probabilistic effects and a certain cost which produces a future state. The purpose of the MDP agent is to minimize the expected cost along a sequence of choices. The number of stages that the agent acts in the environment is called horizon, which can be finite, infinite or undefined. An example of MDP with undefined horizon is the Stochastic Shortest Path MDP, which extends the definition of MDP by adding a set of goal states (the agent stops acting after reaching a goal state). In an SSP MDP the assumption is made that it is always possible to achieve a goal state from every state of the world. However, this is a very strong assumption and cannot be guaranteed in practical applications. States from which it is impossible to reach the goal are called dead-ends. A dead-end may be avoidable or unavoidable (when no policy leads from the initial state to the goal with probability one). Recent work has proposed extensions to SSP MDP that allow the existence of different types of dead-ends as well as algorithms to solve them. However, the detection of dead-end is done using: (i) heuristics that may fail to detect implicitly dead-ends or (ii) more reliable methods that require a high computational cost. In this project we make a formal characterization of probabilistic planning models with dead-ends. In addition, we propose a new technique for dead-end detection based on this characterization and we adapt probabilistic planning algorithms to use this new detection method. The empirical results show that the proposed method is able to detect all dead-ends of a given set of states and, when used with probabilistic planners, can make these planners more efficient in domains with difficult to detect dead-ends.

Keywords: Probabilistic Planning, MDP, SSP MDP, Dead-ends. 


\section{Sumário}

Lista de Figuras $\quad$ xi

Lista de Tabelas $\quad$ xiii

Lista de Algoritmos $\quad$ xv

1 Introdução $\quad 1$

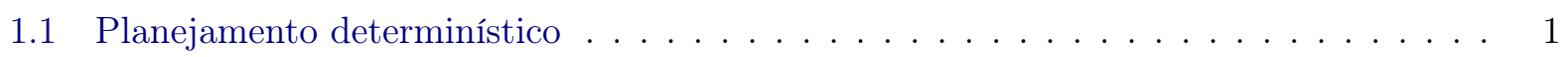

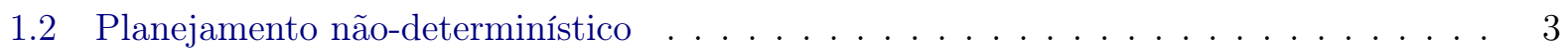

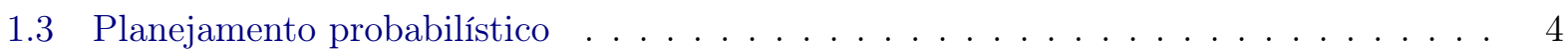

1.4 Planejamento probabilístico com becos-sem-saída . . . . . . . . . . . . . . 5

1.5 Planejando na presença de becos-sem-saída . . . . . . . . . . . . . . . . . 6

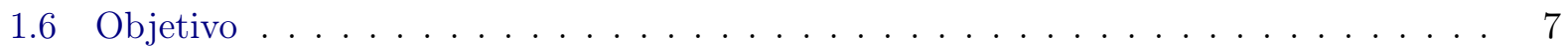

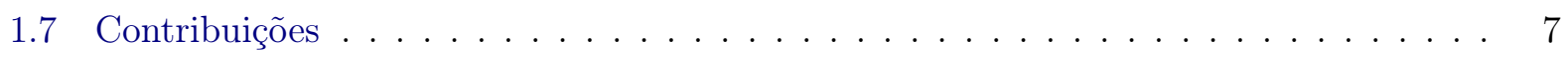

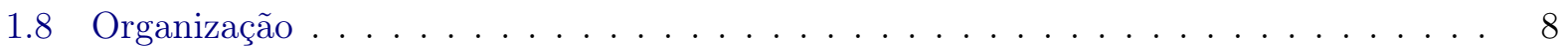

2 Planejamento probabilístico $\quad 9$

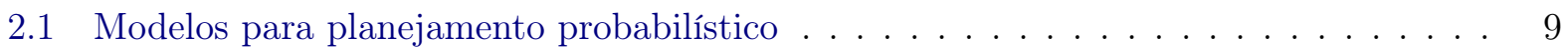

2.1.1 Processos de decisão markovianos . . . . . . . . . . . . . . . . . . . . . . . . 10

2.1 .2 MDP de horizonte infinito . . . . . . . . . . . . . . . . . . 11

2.1 .3 Planejamento orientado à meta . . . . . . . . . . . . . . . . . . . . . . . 12

2.1 .4 MDP de caminho estocástico mínimo . . . . . . . . . . . . . . . 13

2.2 Algoritmos assíncronos de planejamento probabilístico . . . . . . . . . . . . 15

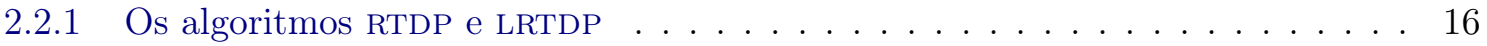

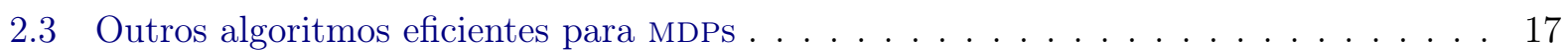

2.4 Representação de um GMDP a partir de uma linguagem de ações . . . . . . . . . . . . 19

2.4.1 Ações determinísticas . . . . . . . . . . . . . . . . . . . . . . . . . . . . . . . . 19

2.4 .2 Ações probabilísticas . . . . . . . . . . . . . . . . . . . . 20

2.4 .3 Ações não-determinísticas . . . . . . . . . . . . . . . . . . . . . . . . . 21

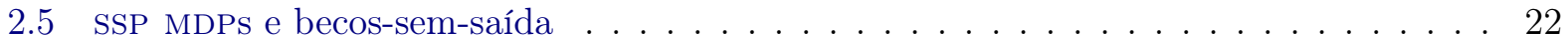

3 Planejamento probabilístico com becos-sem-saída $\quad 23$

3.1 GMDPs com becos-sem-saída . . . . . . . . . . . . . . . . . . . . . 24

3.1.1 GMDPs com becos-sem-saída evitáveis . . . . . . . . . . . . . . . . 24

3.1.2 GMDPs com becos-sem-saída inevitáveis . . . . . . . . . . . . . . . . 25

3.2 Outras classes de GMDPs . . . . . . . . . . . . . . . . . . . . . . 30 
3.2 .1 GMDP com desconto . . . . . . . . . . . . . . . . . . . . . . 30

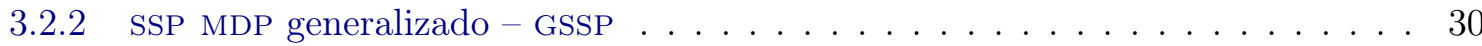

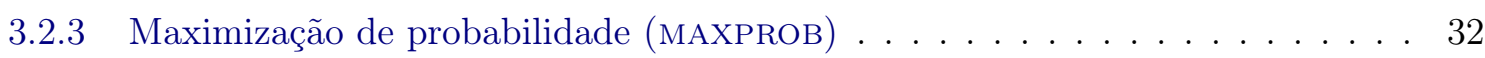

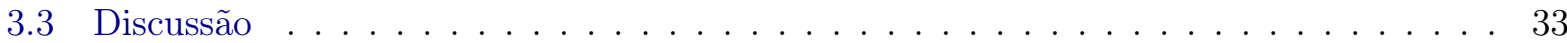

4 Heurísticas baseadas em modelos $\quad 35$

4.1 Heurísticas para planejamento determinístico . . . . . . . . . . . . . . . 37

4.1 .1 Heurísticas $h_{a d d}$ e $h_{\max } \ldots \ldots \ldots \ldots \ldots \ldots \ldots$

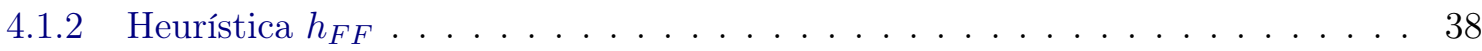

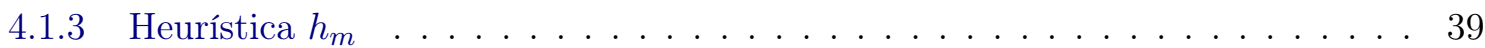

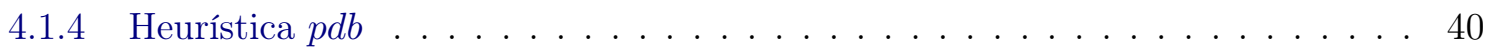

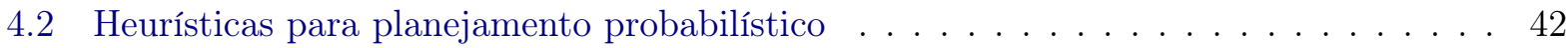

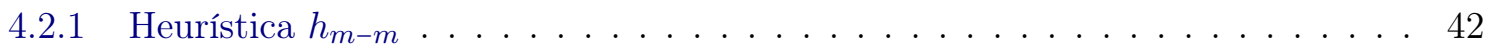

4.2 .2 A heurística do HMdPP . . . . . . . . . . . . . . . . . . . . . 43

4.3 Heurísticas para insolubilidade . . . . . . . . . . . . . . . . . . . 44

4.3 .1 Aprendendo durante a busca . . . . . . . . . . . . . . . . . . 44

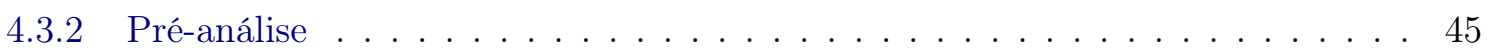

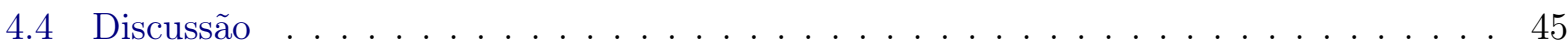

5 Caracterização de GMDPs por verificação de modelos $\quad 47$

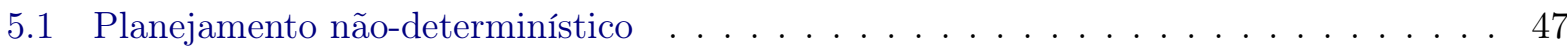

5.2 Verificação de modelos . . . . . . . . . . . . . . . . . . . . . . . . . . . . . 49

5.2 .1 A lógica $\mathrm{CTL} \ldots \ldots \ldots \ldots \ldots \ldots \ldots \ldots$

5.2 .2 Planejamento como verificação de modelos . . . . . . . . . . . . . . . . . 50

5.2.3 $\alpha$-CTL: lógica de tempo ramificado para raciocínio sobre ações $\ldots \ldots \ldots$. . . . . 51

5.3 GMDPs com becos-sem-saída: uma formalização por verificação de modelos . . . . . . 53

5.3.1 Caracterizando o conjunto de becos-sem-saída de um GMDP . . . . . . . . . . . 53

$5.3 .2 \quad$ SSPADE e SSPUDES . . . . . . . . . . . . . . . . . . . . . . 54

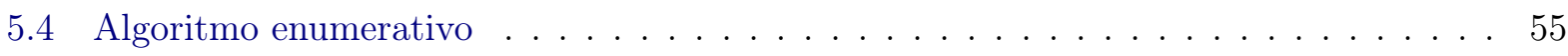

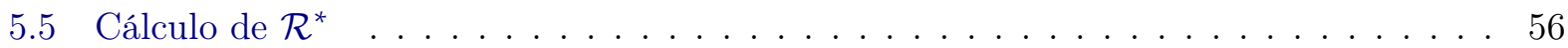

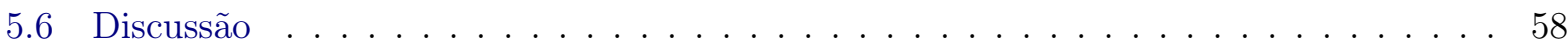

6 Regressão simbólica para detecção de becos-sem-saída $\quad 59$

6.1 Representação simbólica de estados . . . . . . . . . . . . . . . . . . . . . . . . . . 59

6.2 Representação simbólica de ações . . . . . . . . . . . . . . . . . . . . . . 59

6.3 Operações simbólicas: restrição e quantificação existencial . . . . . . . . . . . . . . . 60

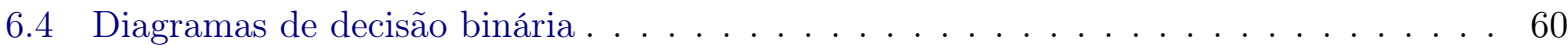

6.5 Regressão simbólica fraca: cálculo do conjunto $\mathcal{R} \ldots \ldots \ldots \ldots \ldots$. . . . . . . . 60

6.6 Um novo detector de becos-sem-saída - SWR-DE . . . . . . . . . . . . . . . 61

7 Análise Empírica $\quad 63$

7.1 Domínios . . . . . . . . . . . . . . . . . . . . . . . 63

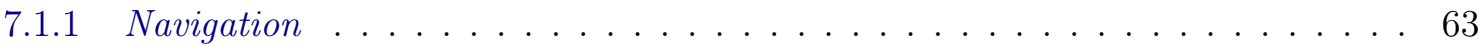

7.1 .2 Triangle TireWorld . . . . . . . . . . . . . . . . . . 65

7.1 .3 Drive ............................ 67 
7.1 .4 Exploding BlocksWorld . . . . . . . . . . . . . . . . . 67

7.2 Completude da deteç̧ão de becos-sem-saída . . . . . . . . . . . . . . . . . . 68

7.2 .1 Detecção de becos-sem-saída no domínio Navigation . . . . . . . . . . . 68

7.2.2 Detecção de becos-sem-saída no domínio Triangle TireWorld . . . . . . . . . . 70

7.2 .3 Detecção de becos-sem-saída no domínio Drive . . . . . . . . . . . . . . . 72

7.2.4 Detecção de becos-sem-saída no domínio Exploding BlocksWorld . . . . . . . . 72

7.2 .5 Detecção de becos-sem-saída: discussão final . . . . . . . . . . . . . . . . 73

7.3 Eficiência do planejamento com detecção de becos-sem-saída . . . . . . . . . . . . . . 74

7.3 .1 Planejamento no domínio Navigation . . . . . . . . . . . . . . 75

7.3.2 Planejamento no domínio Triangle Tireworld . . . . . . . . . . . . . . 77

7.3 .3 Planejamento no domínio Drive . . . . . . . . . . . . . . . 78

7.3.4 Planejamento no domínio Exploding Bolcks World . . . . . . . . . . . . . . . 79

7.3.5 Planejamento com becos-sem-saída: discussão final . . . . . . . . . . . . . 79

8 Conclusão $\quad 81$

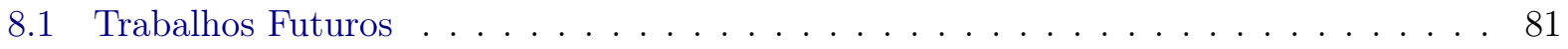

$\begin{array}{ll}\text { A Caminho estocástico mínimo mais seguro } & 83\end{array}$

$\begin{array}{ll}\text { B GMDP com desconto } \times \text { SSP MDP } & 85\end{array}$

$\begin{array}{lll}\text { C } & \text { Algoritmo forte cíclico } & 87\end{array}$

D Especificação de domínios de planejamento probabilístico $\quad \mathbf{8 9}$

D.1 Especificação do domínio Navigation (em RDDL) . . . . . . . . . . . . . . . . 89

D.2 Especificação do domínio Triangle Tire World (em PPDDL) . . . . . . . . . . . . 90

$\begin{array}{ll}\text { Referências Bibliográficas } & 91\end{array}$ 


\section{Lista de Figuras}

1.1 Robô carregador de caixas. . . . . . . . . . . . . . . . . . . . . 1

1.2 Modelo de transição de estados do problema do robô carregador de caixas. . . . . . . 2

1.3 Problema do robô carregador de caixas representado pelos fatos que descrevem o

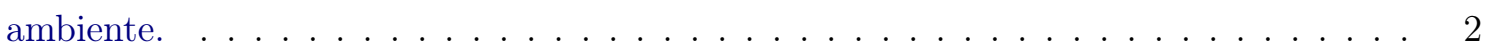

1.4 Representação abstrata do modelo de transição de estados através das ações do robô

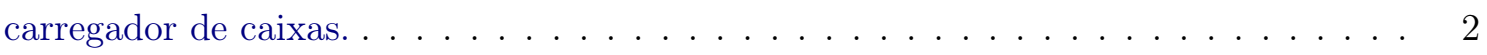

1.5 Representação gráfica das ações move-left e grab (L1) em termos de precondição

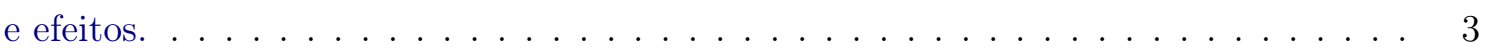

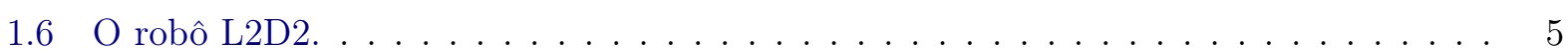

2.1 Interação entre o agente e o ambiente . . . . . . . . . . . . . . . . 9

2.2 Exemplos de GMDPs representados como grafos de transição de estados. . . . . . . . . 13

2.3 Exemplo de grafo guloso $G_{V}$ em um GMdP. . . . . . . . . . . . . . . 13

2.4 Exemplo de GMDP com política própria e políticas impróprias . . . . . . . . . . . . 15

2.5 Exemplo de trial do algoritmo RTDP. . . . . . . . . . . . . . . . . . . . 17

2.6 Especificação da ação de mover de $l o c_{2}$ para $l o c_{g}$ do robô L2D2 (Figura 1.6). . . . . . 20

2.7 Interpretação do problema de planejamento como um modelo de transição de estados. 21

2.8 Exemplos de GMDPs que não satisfazem as suposições do SSP MDP. . . . . . . . . . . . 22

3.1 Exemplo de GMDP com becos-sem-saída . . . . . . . . . . . . . . . . . . . . . 23

3.2 Problemas com becos-sem-saída evitáveis e inevitáveis. . . . . . . . . . . . . . . . 24

3.3 Relação entre ssP MdPs e SSPADE MDPs. . . . . . . . . . . . . . . . . . . 25

3.4 Utilidade das melhores políticas de um problema multiobjetivo. . . . . . . . . . . . 27

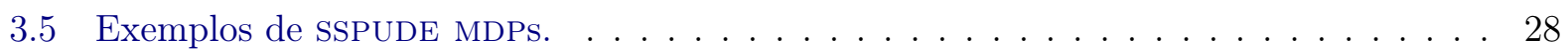

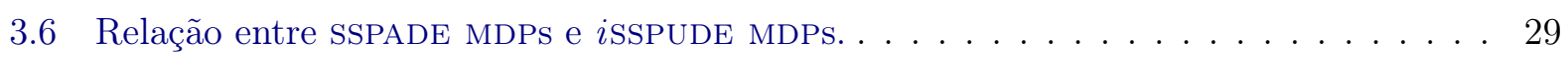

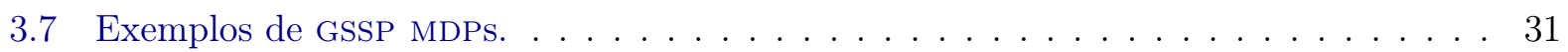

3.8 Exemplos de GMDPs que não satisfazem as suposições dos GSSP MDPs. . . . . . . . . . 31

4.1 Exemplos de determinização. . . . . . . . . . . . . . . . . . 36

4.2 Grafo de planejamento relaxado. . . . . . . . . . . . . . . . . . . . . 39

4.3 Exemplos de projeções. . . . . . . . . . . . . . . . . . . . . . 41

4.4 Determinização de laço próprio . . . . . . . . . . . . . . . . . . . . . . . 43

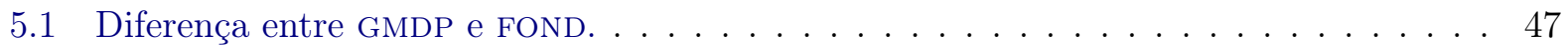

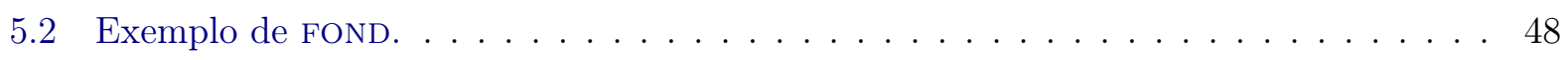

5.3 Semântica dos operadores temporais da lógica CTL [Pereira, 2007]. . . . . . . . . . . 50 
5.4 Estrutura de Kripke rotulada $\mathcal{D}$ e estrutura de Kripke $\mathcal{K}(\mathcal{D}) \ldots \ldots \ldots \ldots$. . . . . . 52

5.5 Semântica dos operadores temporais da lógica $\alpha$-CTL [Menezes, 2014] . . . . . . . . . 53

5.6 Relação entre os tipos de GMDPs . . . . . . . . . . . . . . . . . . . . . . . 55

5.7 Funcionamento do algoritmo weakPReImageFixpoint . . . . . . . . . . . . 56

5.8 Computação do ponto fixo da fórmula $\phi=\forall \bullet \exists \diamond \psi \ldots \ldots \ldots \ldots \ldots \ldots$

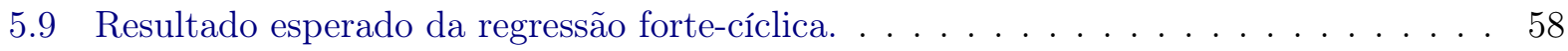

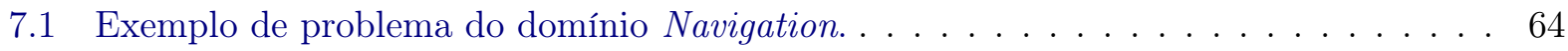

7.2 Duas instâncias do domínio Triangle TireWorld. . . . . . . . . . . . . . 66

7.3 Tempo gasto para avaliar todos os estados de 16 instâncias do domínio Navigation_0. 69

7.4 Tempo gasto para avaliar todos os estados em 16 instâncias do domínio Navigation_1. 69

7.5 Tempo gasto para avaliar todos os estados em 16 instâncias do domínio Navigation_2. 70

7.6 Tempo gasto para avaliar todos os estados no domínio Triangle Tire World_ $0 \ldots \ldots$. . 71

7.7 Tempo gasto para avaliar todos os estados no domínio TriangleTireWorld_ 1 . . . . . 72

7.8 Tempo gasto para avaliar todos os estados no domínio Drive. . . . . . . . . . 73

7.9 Tempo gasto para avaliar todos os estados no domínio ExplodingBlocks World. . . . . 73

7.10 Tempo de planejamento (segundos) no domínio Navigation_0 . . . . . . . . 75

7.11 Tempo de planejamento (segundos) no domínio Navigation_ 1 . . . . . . . . . . 76

7.12 Tempo de planejamento (segundos) no domínio Navigation_2. . . . . . . . . . . 77

7.13 Tempo de planejamento (segundos) no domínio TriangleTireworld_o $\ldots \ldots \ldots$

7.14 Tempo de planejamento (segundos) no domínio TriangleTireWorld_ $\ldots \ldots$. . . . 78

7.15 Tempo de planejamento (segundos) no domínio Drive . . . . . . . . . . . . . . . 79

7.16 Tempo de planejamento (segundos) no domínio ExplodingBlocksWorld . . . . . . . . . 79

B.1 Exemplo de GMDP parametrizado com duas políticas. . . . . . . . . . . . . . . 85

B.2 Impacto do fator de desconto no GMDP da Figura B.1 no valor de $n$ (Equação B.1) . 86 


\section{Lista de Tabelas}

6.1 Correspondência entre operadores lógicos e operações entre BDDs. . . . . . . . . . . . . 60

7.1 Estatísticas para 16 instâncias do domínio Navigation_0 . . . . . . . . . . . . 64

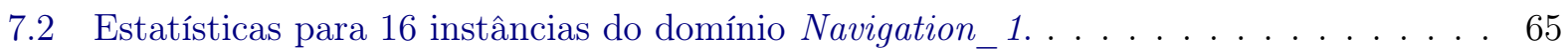

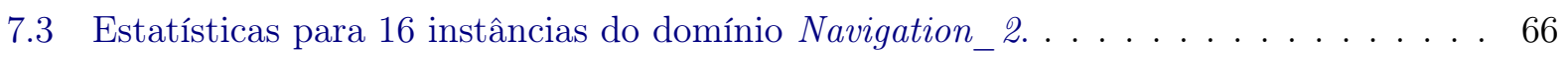

7.4 Estatísticas de 5 instâncias do domínio Triangle TireWorld_ 0 . . . . . . . . . . . 66

7.5 Estatísticas de 5 instâncias do domínio Triangle TireWorld_ 1 . . . . . . . . . . . 67

7.6 Estatísticas de 15 instâncias do domínio Drive. . . . . . . . . . . . . . . . . . 67

7.7 Estatísticas de 4 instâncias do domínio ExplodingBlocks World. . . . . . . . . . . . . . 68

7.8 Número de becos-sem-saída detectados por cada heurística e pelo swR-DE em 16

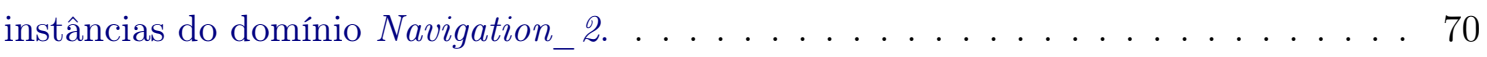

7.9 Número de becos-sem-saída detectados por cada heurística e pelo SWR-DE no domínio

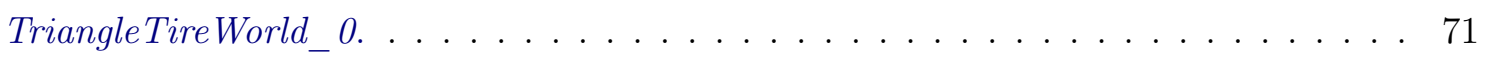

7.10 Número de becos-sem-saída detectados por cada heurística e pelo SWR-DE no domínio Triangle TireWorld_1. . . . . . . . . . . . . . . . . 71

7.11 Número de becos-sem-saída detectados por cada método em 15 instâncias do domínio

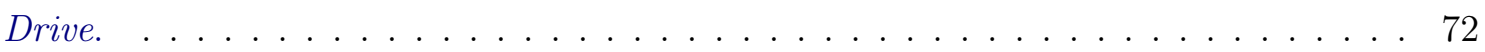

7.12 Número de becos-sem-saída detectados por cada método em 4 instâncias do domínio

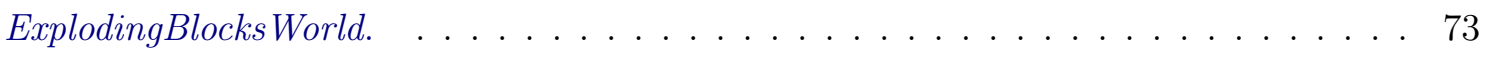

7.13 Como os planejadores analisados resolvem problemas com becos-sem-saída. . . . . . 75 


\section{Lista de Algoritmos}

2.1 Esquema Find and Revise[Geffner e Bonet, 2013] . . . . . . . . . . . . . . . 16

2.2 O algoritmo LRTDP [Bonet e Geffner, 2003b] . . . . . . . . . . . . . . . . 18

2.3 O algoritmo ILAO $^{*}[$ Hansen e Zilberstein, 2001] . . . . . . . . . . . . . . . . . . . 19

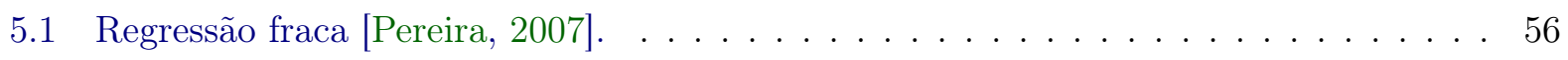

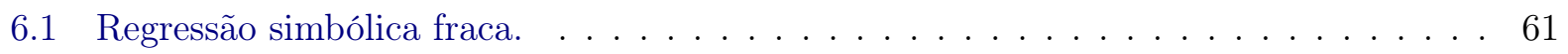

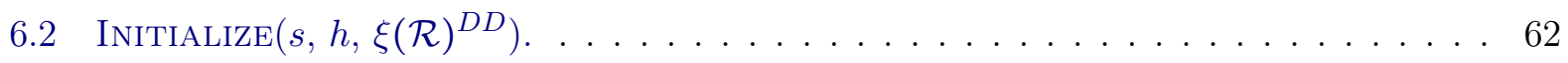

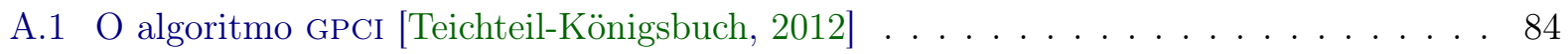

C.1 $\operatorname{StrongCYCLICPlAn}(\mathcal{M}, \mathbb{G}) \rightarrow \pi \ldots \ldots \ldots \ldots$ 


\section{Capítulo 1}

\section{Introdução}

O foco de estudo da área de Inteligência Artificial (IA) é o desenvolvimento de agentes inteligentes, isto é, agentes capazes de perceber o mundo e atuar nele, de forma a maximizar sua função de utitidade. Um dos aspectos fundamentais de um agente inteligente é a capacidade de planejar tarefas de forma autônoma. Planejamento automatizado [Russell e Norvig, 2010], um dos mais antigos ramos da IA, se preocupa em propor técnicas computacionais de raciocínio de ações e algoritmos eficientes para o planejamento.

\subsection{Planejamento determinístico}

Entre as sub-áreas do planejamento automatizado, o planejamento clássico estuda ambientes determinísticos, completamente observáveis e com a presença de um único agente, tendo como objetivo encontrar um plano composto por uma sequência de ações que leva o agente de um estado inicial para um estado meta. A Figura 1.1 mostra um exemplo de problema de planejamento clássico. Neste problema, um robô está em um ambiente com duas salas e uma caixa. Este robô é capaz de se mover entre as salas (move-left e move-right), pegar (grab) e soltar (drop) a caixa. A meta é que a caixa esteja na sala da direita, sendo que no estado inicial o robô e a caixa estão na sala da esquerda e a caixa está no chão. Um plano solução é grab, move-right e drop.

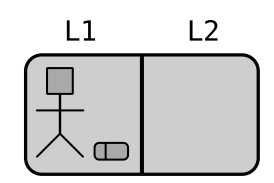

Figura 1.1: Robô carregador de caixas.

A dinâmica do ambiente pode ser descrita por um modelo de transição de estados [Ghallab et al., 2004, 2016; Russell e Norvig, 2010], que pode ser representado de forma explícita por um grafo dirigido, no qual o conjunto de vértices $S$ representa os estados e o conjunto de arestas $A$ representa as possíveis ações que o agente pode aplicar (chamaremos esse grafo de grafo de transição de estados). Dessa forma, um problema de planejamento pode ser visto como um problema de caminho mínimo no grafo de transição de estados: dados dois vértices $s \in S$ (estado inicial) e $s^{\prime} \in S$ (estado meta), deve-se encontrar o menor caminho, isto é, uma sequência de arestas (plano) que liga o vértice $s$ ao vértice $s^{\prime}$. A Figura 1.2 mostra o grafo de transição de estados do problema do robô, em que o vértice $s_{1}$ representa o estado em que o robô está na sala da direita e a caixa está no chão da sala da esquerda, o vértice $s_{2}$ representa o estado em que o robô está na sala da esquerda e a caixa está no chão da sala da esquerda e assim por diante. As arestas indicam uma transição de estado, por exemplo, quando o agente aplica a ação move-left no estado $s_{1}$, o ambiente muda para o estado $s_{2}$.

Os estados $s_{1}, s_{2}, \cdots, s_{n}$ podem ser representados através de um conjunto de fatos que descrevem as propriedades do mundo, denotamos esse cojunto por $\mathbb{P}$. No exemplo do robô, um estado pode 


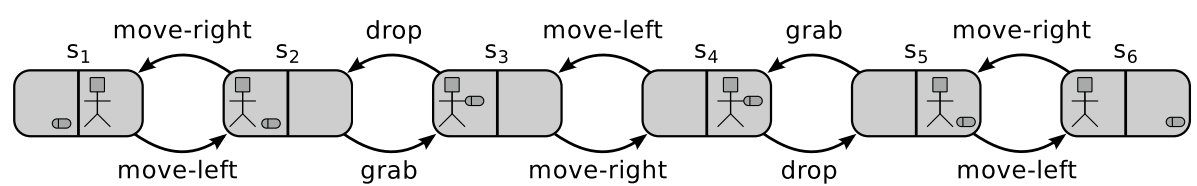

Figura 1.2: Modelo de transição de estados do problema do robô carregador de caixas.

ser descrito apenas pela localização do robô (robot-at (L1) ou robot-at (L2) ) e a posição da caixa, que pode estar em uma das localizações (box-at (L1) ou box-at (L2)) ou sendo segurada pelo robô (holding-box). Assim, um estado pode ser representado apenas pelo conjunto dos fatos que são verdadeiros nele (Closed-world assumption - CWA). A Figura 1.3 mostra como podemos representar os estados do problema do robô através de conjuntos de fatos verdadeiros. Neste exemplo, o estado \{robot-at ( L1), box-at (L1) \} representa um estado em que o robô e a caixa estão na sala L1, vértice $s_{2}$ (Figura 1.2).

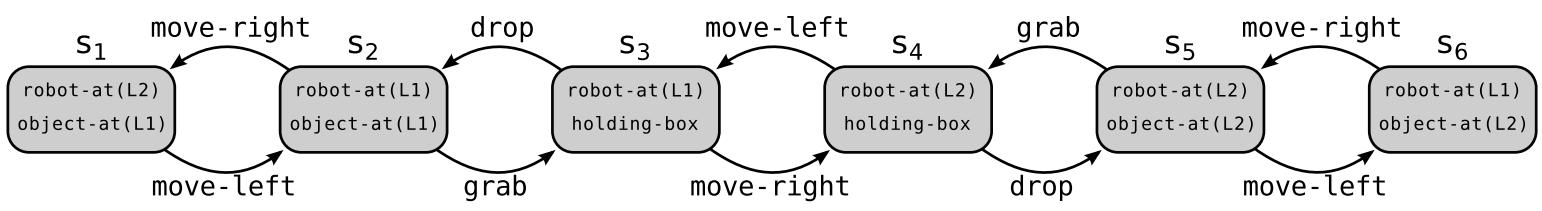

Figura 1.3: Problema do robô carregador de caixas representado pelos fatos que descrevem o ambiente.

O modelo de transição de estados pode ser representado de forma ainda mais compacta. Por exemplo, a Figura 1.4 representa o problema do robô carregador de caixas através de estados abstratos (cada estado abstrato representa um conjunto de estados). Na Figura 1.4(a) percebemos que a posição da caixa é irrelevante para as ações move-right e move-left, uma vez que se o robô estiver segurando a caixa ela se move com o robô, caso contrário, a caixa permanece onde estava. Essas ações representam de forma abstrata as ligações entre os vértice $s_{1}$ e $s_{2}$ do sistema de transição da Figura 1.2, bem como as transições entre $s_{4}$ e $s_{3}$ e as transições entre $s_{5}$ e $s_{6}$. De forma similar, as ações de pegar e soltar a caixa só dependem que o agente esteja na mesma posição que a caixa (Figura 1.4(b)), e elas representam as transições entre os vértices $s_{2}$ e $s_{3}$, bem como $s_{5}$ e $s_{4}$. Note que, a posição do agente e do ambiente é representada em primeira ordem pela variável $X$, podendo ser instanciada pelas localizações L1 e L2. O uso de variáveis de primeira ordem permite uma representação ainda mais compacta dos problemas de planejamento. Por simplicidade, neste trabalho assumiremos que as ações são proposicionais, isto é, sem variáveis de primeira ordem.

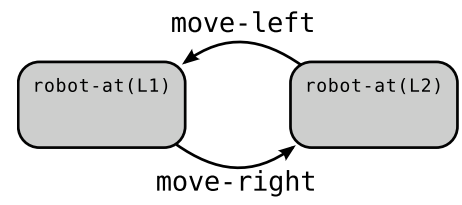

(a)

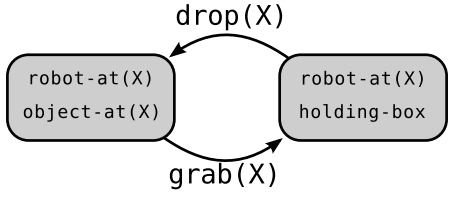

(b)

Figura 1.4: Representação abstrata do modelo de transição de estados através das ações do robô carregador de caixas: (a) move-right e move-left; (b) grab (X) e drop (X).

Dessa forma, o uso de uma linguagem de descrição de ações para descrever um domínio de planejamento permite a especificação de problemas de planejamento de forma mais compacta. A linguagem STRIPS [Fikes e Nilsson, 1971] descreve cada ação através de três conjuntos de fatos: (i) precondição, conjunto de fatos que devem ser verdadeiros em um estado para que uma ação possa ser aplicada; (ii) efeitos de adição, conjunto de fatos que se tornam verdadeiros após a ação ser aplicada; e (iii) efeitos de eliminação, conjunto de fatos que se tornam falsos após a ação ser aplicada. A Figura 1.5 mostra uma representação gráfica das ações move-left e grab (L1). Observe que a ação move-left não diz nada sobre a posição em que a caixa se encontra: se a 
caixa estiver no chão ela permanecerá lá, se o robô estiver segurando a caixa ela se moverá também. Denotamos o conjunto de ações de um problema por $\mathbb{A}$.

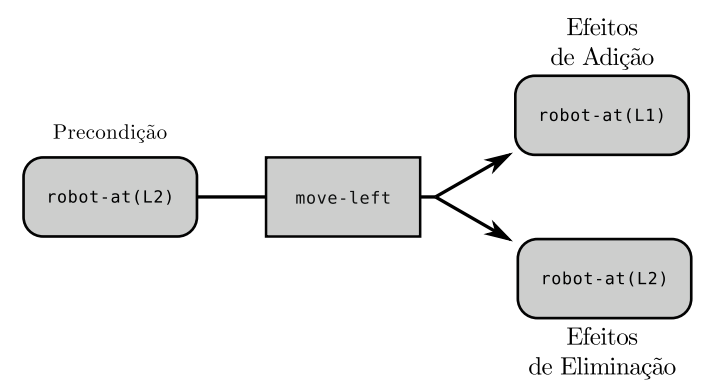

(a)

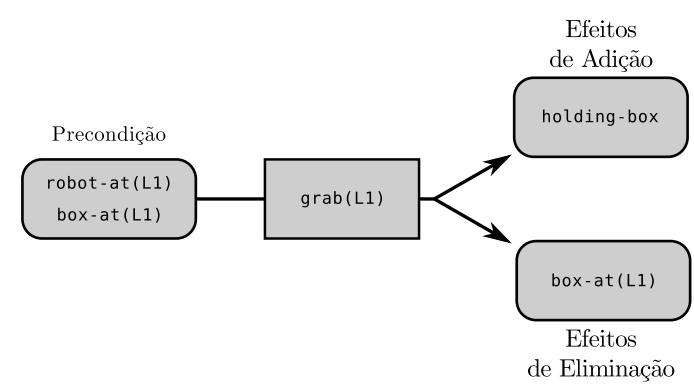

(b)

Figura 1.5: Representação gráfica das ações move-left e grab(L1) em termos de precondição e efeitos.

Neste trabalho, utilizaremos o símbolo $\mathcal{M}$ para denotar um problema descrito por um modelo de transição de estados (grafo direcionado $\langle S, A\rangle$ ) e o símbolo $\mathcal{P}$ para denotar problemas descritos através de uma linguagem de descrição de ações (em função de um conjunto de fatos e um conjunto de ações $\langle\mathbb{P}, \mathbb{A}\rangle)$.

Uma das principais características da área de planejamento é que os planejadores se baseiam em linguagens de especificação de ações para raciocinar sobre o mundo, explorando assim a estrutura do problema para encontrar um plano sem precisar expandir todo o grafo de transição de estados.

De uma forma geral, planejadores clássicos se dividem em duas classes de algoritmos: planejadores que fazem busca no espaço de planos e busca no espaço de estados. O planejador de ordem-parcial (POP) [Chapman, 1987], por exemplo, realiza uma busca no espaço de planos, isto é, uma busca em um grafo em que cada vértice é um plano parcial, representando um conjunto de planos. Planejadores automáticos que fazem busca heurística no espaço de estados são considerados estado da arte em planejamento clássico [Bonet e Geffner, 2001]. Nesse caso, o planejador faz uma busca no espaço de estados e usa uma versão relaxada do problema para estimar a distância para um estado meta. Essas mesmas heurísticas também são usadas em planejamento não-clássico, conforme veremos a seguir.

O planejador FF (Fast-Forward) [Hoffmann, 2001], um dos planejadores mais bem sucedidos nas competições internacionais de planejamento, utiliza busca heurística para encontrar um plano ótimo (caso a heurística seja admissível) para problemas envolvendo até $10^{20}$ estados [Edelkamp, 2000]. Existem outras classes de planejadores automáticos, entre elas o planejador Graphplan [Blum e Furst, 1997], usado no cálculo da heurística do FF. O Graphplan faz uma busca no espaço híbrido de planos e estados (como descreveremos no Capítulo 4).

Apesar do planejamento determinístico possuir soluções eficientes, as suposições restritivas que são feitas podem ser incompatíveis com muitos problemas reais. Em especial, a suposição de que o ambiente é determinístico; e que não existem outros agentes atuando no mundo ou a ocorrência de eventos exógenos.

\subsection{Planejamento não-determinístico}

O planejamento não-determinístico lida com ambientes completamente (totalmente) ou parcialmente observáveis ${ }^{1}$, em que existe incerteza nos efeitos das ações, mas não existem probabilidades associadas a esses efeitos. Esses problemas são modelados através de ações com efeitos não-determinísticos.

A solução para esses problemas é dada por uma política, função que mapeia estados em ações. Uma política define qual ação deverá ser aplicada a cada estado visitado.

\footnotetext{
${ }^{1}$ Nesse trabalho assumimos que o ambiente é totalmente observável.
} 
Em planejamento não-determinístico, a qualidade de uma política pode ser de três tipos:

Fraca: política que alcança um estado meta, mas não tem garantias;

Forte: política que garante alcançar um estado meta em um número finito de passos; e

Forte-cíclica: política que garante em algum momento alcançar um estado meta.

\section{Exemplos de soluções para planejamento não-determinístico.}

- O planejamento como verificação de modelos é um meio de resolver problemas de planejamento não-determinístico. Esse método se baseia em técnicas formais de verificação de modelos para sintetizar uma política.

- Soluções de replanejamento usam um planejador clássico para computar um plano em uma versão relaxada (determinística) do problema, ignorando a incerteza dos efeitos das ações. Se durante a execução desse plano o agente encontrar um estado inesperado, ele replaneja partindo desse novo estado.

\subsection{Planejamento probabilístico}

Planejamento probabilístico envolve problemas em que os efeitos não-determinísticos das ações têm probabilidades associadas, e são geralmente modelados por processos de decisão markovianos (Markovian Descision Processes - MDPs) [Puterman, 1994]. MDPs são definidos por um conjunto de estados $S$, um conjunto de ações $A$, uma função custo $C$ e uma função de transição de estados probabilística $P$. A política $\pi$ que se deseja encontrar mapeia estados em ações, isto é $\pi: S \rightarrow A$. Um MDP modela a interação entre um agente e o seu ambiente: em cada estágio, o agente se encontra em um estado (completamente observável) e decide executar uma ação (com efeitos probabilísticos) que irá produzir um estado futuro e um custo. O número de estágios que o agente atua no ambiente é chamado de horizonte, o qual pode ser finito, infinito ou indefinido. O objetivo do agente é minimizar o custo esperado ao longo de um horizonte.

Outro modelo para planejamento probabilístico é o MDP de Caminho Estocástico Mínimo (Stochastic Shortest Path MDP - SSP MDP) [Bertsekas e Tsitsiklis, 1991]. Um SSP MDP é um MDP em que são conhecidos um conjunto de estados meta $G$ e um conjunto de estados iniciais $S_{0} \subseteq S$. Num SSP MDP o agente para de agir ao alcançar um estado meta e, dessa forma, o horizonte de atuação do agente é indefinido.

Soluções baseadas em programação dinâmica. O algoritmo Iteração de Valor (Value Iteration - VI) é um método clássico para resolver MDPs e SSP MDPs. Essa abordagem computa uma política ótima, que minimiza o custo esperado do agente utilizando técnicas de programação dinâmica [Puterman, 1994]. Isso é feito calculando-se aproximações sucessivas de uma função valor de estado que representa o quão bom para o agente é estar em um determinado estado até a convergência desse valor. A cada iteração, todos os estados são atualizados simultaneamente, por esta razão este algoritmo utiliza programação dinâmica sincrona [Puterman, 1994]. A complexidade do algoritmo vi é $O(|S| \times|A| \times|S|)$. Se considerarmos que estados são descritos por um conjunto $\mathbb{P}$ de variáveis booleanas, temos que $|S|=2^{|\mathbb{P}|}$, ou seja, o número de estados em um MDP cresce exponencialmente em função do número de variáveis de estado. Esse fato é conhecido como maldição da dimensionalidade de Bellman [Bellman, 1957].

A programação dinâmica síncrona, que aproxima o valor de todos os estados possíveis a cada iteração, não é uma estratégia escalável [Bonet e Geffner, 2003b; Hansen e Zilberstein, 2001]. Algoritmos de programação dinâmica assíncrona atualizam apenas um subconjunto do espaço de estados a cada iteração, dando mais prioridade a estados com maior probabilidade de serem alcançados. Dessa forma, estas técnicas são capazes de resolver problemas maiores, porém, ainda assim soluções eficientes dependem do emprego de boas heurísticas para a aproximação inicial do valor de cada 
estado. Como veremos nesse trabalho, essas heurísticas são as mesmas heurísticas utilizadas no planejamento clássico, fazendo-se uma determinização (relaxação) do MDP ou SSP MDP.

\subsection{Planejamento probabilístico com becos-sem-saída}

O modelo de planejamento probabilístico SSP MDP faz a suposição de que é possível alcançar um estado meta a partir de qualquer estado $s \in S$. Portanto, SSP MDPs não modelam problemas envolvendo estados a partir dos quais o agente é incapaz de alcançar a meta, estados chamados de becos-sem-saída. Os algoritmos citados anteriormente assumem que a entrada é um SSP MDP, ignorando a existência de becos-sem-saída, o que faz eles perderem suas garantias de convergência, podendo falhar, ou devolver políticas indesejadas. Para resolver problemas com becos-sem-saída é necessário definir novos modelos que permitam a presença desses estados, bem como desenvolver algoritmos para solucionar tais problemas. Trabalhos recentes [Kolobov et al., 2011, 2012b; Teichteil-Königsbuch, 2012; Teichteil-Königsbuch et al., 2011] têm proposto extensões de SSP MDPs que lidam com essas situações.

Exemplo 1 (Robô L2D2). Na Figura 1.6 o robô L2D2 está em um edifício de dois andares (térreo e subsolo) e deve percorrer os andares e salas para realizar suas tarefas. Existe uma escada que conecta os dois andares. L2D2 pode se mover entre as salas conectadas por portas ou descer as escadas. Note que para L2D2 ir de loc $_{0}$ para loc 3 é necessário que a porta entre estas salas esteja aberta (Figura 1.6(a)), caso contrário (Figura 1.6(b)), não é possível realizar essa ação.

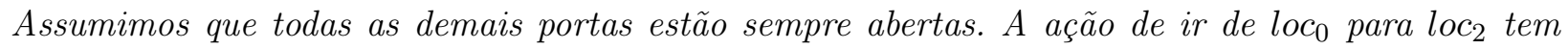
efeitos probabilísticos e pode levar L2D2 para a sala loc $_{2}$ (com probabilidade 0.8) ou para a escada loc 1 (com probabilidade 0.2), o que leva L2D2 para o subsolo (Figura 1.6(c)). Inicialmente, L2D2 está na sala loc $_{0}$ e sua meta é chegar à sala loc . Como L2D2 é incapaz de subir escadas, caso chegue ao subsolo, ele será incapaz de chegar à meta. Portanto, os estados em que L2D2 encontra-se no subsolo são becos-sem-saída.

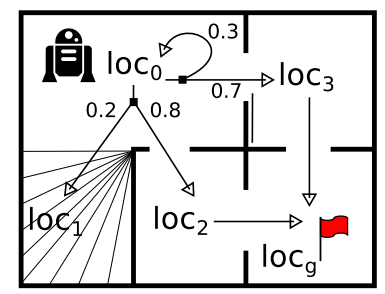

(a)

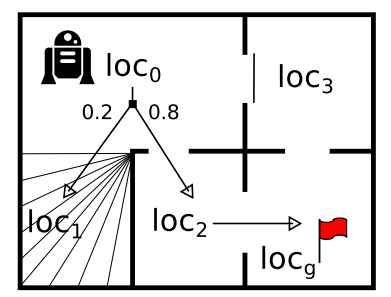

(b)

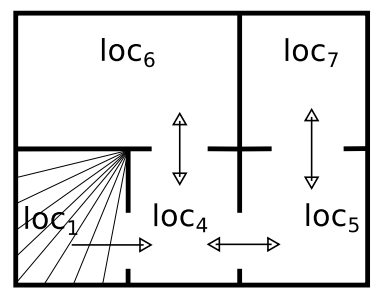

(c)

Figura 1.6: $O$ robô L2D2 está na sala loc $_{0}$ de um edifício de dois andares e precisa chegar à

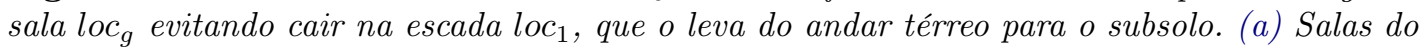

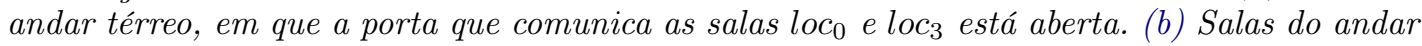
térreo, em que a porta que comunica as salas $\operatorname{loc}_{0}$ e loc está fechada. (c) Salas e escada do subsolo.

Problemas com becos-sem-saída podem ser divididos em duas classes:

Problemas com becos-sem-saída evitáveis: Um problema tem becos-sem-saída evitáveis se, partindo do estado inicial, existe uma forma de alcançar a meta com probabilidade 1, ou seja, sem nenhum risco de alcançar um beco-sem-saída;

Problemas com becos-sem-saída inevitáveis: Um problema tem becos-sem-saída inevitáveis quando todas as trajetórias que levam do estado inicial para algum estado meta têm alguma chance de alcançar um beco-sem-saída, ou seja, toda política tem probabilidade menor que 1 de levar o agente à meta. 
No Exemplo 1, quando a porta encontra-se aberta (Figura 1.6(a)) o agente pode optar por chegar à meta passando pela sala $l o c_{3}$. Essa rota permite que L2D2 chegue à $l o c_{g}$ sem nenhum risco de cair na escada. Assim, notamos que nesse caso $l o c_{1}$ é um beco-sem-saída evitável. Quando a porta encontra-se fechada (Figura 1.6(b)) o agente só tem a opção de tentar chegar à meta passando pela sala $l o c_{2}$. Como a ação ir-para-loc2 pode levar o agente para a escada, esse trajeto oferece uma probabilidade de cair em um beco-sem-saída. Nesse caso, este problema possui becos-sem-saída inevitáveis.

Os becos-sem-saída são divididos em dois tipos:

Becos-sem-saída explícitos: Um beco-sem-saída $d$ é um estado absorvente que não faz parte do conjunto de estados meta. Becos-sem-saída explícitos são facilmente detectáveis.

Becos-sem-saída implícitos: Um beco-sem-saída $d$ é dito implícito se possuir uma ou mais ações aplicáveis que podem levá-lo para outros becos-sem-saída. Em geral, becos-sem-saída implícitos não são facilmente detectáveis.

No Exemplo 1, os estados em que L2D2 está no subsolo e ainda é capaz de se mover tratam-se de becos-sem-saída implícitos. Caso L2D2 quebre depois de descer as escadas, o que implica que todas as ações deixarão o agente no mesmo estado, corresponde a um beco-sem-saída explícito.

\subsection{Planejando na presença de becos-sem-saída}

Alguns algoritmos de programação dinâmica assíncrona, como o (L)RTDP [Barto et al., 1995; Bonet e Geffner, 2003b], se baseiam na execução de trials (simulações que partem do estado inicial e param quando alcançam um estado meta). Na presença de becos-sem-saída, pode ser que um trial nunca chegue a um estado meta, assim o (L)RTDP pode falhar. No Exemplo 1, quando L2D2 chega ao subsolo, ele pode continuar se movimentando infinitamente, logo um trial do (L)RTDP pode ter tamanho infinito, o que resultaria em falha.

O algoritmo ILAO* [Hansen e Zilberstein, 2001] tem uma característica um pouco diferente do LRTDP. Esse algoritmo visita todos os estados que podem ser alcançados a partir de uma dada política e evita a repetição de estados. Assim, o ILAO ${ }^{*}$ não entra em um laço infinito quando alcança um beco-sem-saída e consegue resolver problemas com becos-sem-saída evitáveis sem nenhuma alteração. Uma vez que o valor esperado dos becos-sem-saída continua aumentando, o custo dos outros estados irá convergir para valores finitos, levando o ILAO * a optar por uma política que evite os becos-sem-saída. Por outro lado, o ILAO ${ }^{*}$ não converge no caso de problemas com becos-sem-saída inevitáveis, uma vez que o custo esperado do estado inicial é infinito.

Apesar dos algoritmos de planejamento probabilístico poderem ser adaptados para tratar problemas com becos-sem-saída, o tempo de execução desses algoritmos depende do tempo que eles levam para detectar becos-sem-saída [Kolobov et al., 2010, 2012b]. No Exemplo 1, depois que o agente percebe que os estados do subsolo tratam-se de becos-sem-saída, o agente pode economizar tempo de processamento e memória ignorando estes estados. Portanto, a detecção eficiente de becossem-saída pode reduzir o custo computacional necessário para resolver problemas e incrementar a qualidade das soluções. Além disso, no caso de becos-sem-saída inevitáveis, esses algoritmos podem ser modificados para encontrar soluções que maximizem a probabilidade de alcançar um estado meta, enquanto minimizam o custo esperado das trajetórias que alcançam um estado meta.

A detecção de becos-sem-saída também é relevante para resolver problemas de planejamento determinístico. Em especial, a detecção de becos-sem-saída pode ser utilizada para verificar se um problema determinístico tem solução, analisando se o estado inicial é um beco-sem-saída. Por muito tempo, as competições internacionais de planejamento concentraram-se apenas em problemas que possuíam solução. Com isso, os planejadores assumiam que os problemas de entrada possuíam uma solução, podendo perder muito tempo se não houvesse. Em 2016, surgiu uma nova competição de planejamento, a unsolvability IPC, cuja proposta é testar a habilidade dos planejadores em reconhecer um problema sem solução. 
Observamos portanto, que o problema de deteç̧ão de becos-sem-saída ainda é um assunto pouco explorado e muito relevante para toda a comunidade de planejamento automatizado. Em especial, poucos trabalhos têm se preocupado em estudar tal problema no âmbito de planejamento probabilístico e ainda existem muitas técnicas a serem exploradas nessa área.

Em suma, ao lidar com problemas de planejamento com becos-sem-saída é essencial a utilização de métodos eficientes para detecção de becos-sem-saída que permitam aos planejadores reduzir o consumo de tempo e memória, sem perder a garantia de encontrar a melhor solução.

\subsection{Objetivo}

O objetivo desse trabalho de mestrado é propor a caracterização das diferentes classes de problemas de planejamento probabilístico com becos-sem-saída, bem como o conjunto de becos-sem-saída de um dado problema de planejamento probabilístico, utilizando um arcabouço formal de verificação de modelos. Além disso, propomos o desenvolvimento de um algoritmo eficiente de detecção de becos-sem-saída baseado nessa caracterização e em técnicas de verificação de modelos simbólica. Esse algoritmo é avaliado empiricamente e comparado com heurísticas existentes da área.

\subsection{Contribuições}

As principais contribuições desse trabalho de mestrado são:

- Caracterização de modelos de planejamento probabilístico com becos-sem-saída e do conjunto de becos-sem-saída de um problema de planejamento probabilístico. Esse foi o primeiro trabalho que propôs o uso de um arcabouço formal para caracterização de becos-sem-saída e modelos de planejamento probabilístico com becos-sem-saída.

- Desenvolvimento de uma nova técnica para detecção de becos-sem-saída baseada na caracterização de problemas de planejamento com becos-sem-saída e em técnicas de verificação de modelos simbólica, chamada detector por regressão simbólica fraca (Symbolic Weak Regression Detector - SWR-DE). Os resultados empíricos mostram que o método proposto é capaz de detectar todos os becos-sem-saída de um dado conjunto de estados.

- Adaptação dos algoritmos de planejamento probabilístico para utilização do novo método de deteç̧ão, o SWR-DE.

- Análise comparativa da eficiência de detecção de becos-sem-saída das heurísticas e do método SWR-DE. Os resultados empíricos mostram que, quando usado com planejadores probabilísticos, o método proposto pode tornar esses planejadores mais eficientes em domínios com becos-sem-saída difíceis de serem detectados.

Parte do trabalho apresentado nessa dissertação geraram os seguintes artigos:

- Thiago Dias Simão, Leliane Nunes de Barros e Felipe Leno da Silva. Planejamento Probabilístico com Becos Sem Saída. Em XII Encontro Nacional de Inteligência Artificial (ENIAC 2015), Natal, Brasil.

- Thiago Dias Simão, Ignasi Andres, Viviane Bonadia dos Santos e Leliane Nunes de Barros. Heurísticas para Detecção de Becos sem Saída em Planejamento Probabilístico. Em XIII Encontro Nacional de Inteligência Artificial (ENIAC 2016), Recife, Brasil.

- Thiago Dias Simão, Leliane Nunes de Barros, Silvio do Lago Pereira. Planning with Dead-Ends via Model Checking. (Em elaboração.) 


\subsection{Organização}

Esta dissertação está organizada da seguinte forma:

Capítulo 2: Apresenta os fundamentos para planejamento probabilístico: os modelos MDP e SSP MDP, além dos algoritmos para resolução desses problemas;

Capítulo 3: Descreve modelos para planejamento probabilístico que lidam com becos-sem-saída;

Capítulo 4: Apresenta diversas heurísticas que podem ser utilizadas em planejamento probabilístico, além de heurísticas que podem ser utilizadas para detecção de becos-sem-saída;

Capítulo 5: Caracteriza os modelos para planejamento probabilístico com becos-sem-saída utilizando métodos formais de planejamento (não-determinístico) baseado em verificação de modelos;

Capítulo 6 Apresenta um algoritmo simbólico baseado em métodos de verificação de modelos simbólica para detecção de becos-sem-saída;

Capítulo 7: Realiza uma análise de problemas de planejamento probabilístico com becos-semsaída; analisa o desempenho de heurísticas quando aplicadas ao problema de detecção de becos-sem-saída e mostra uma análise empírica do uso do método de detecção de becos-semsaída proposto (o SWR-DE) nos algoritmos de planejamento probabilístico.

Capítulo 8: Apresenta as conclusões deste trabalho. 


\section{Capítulo 2}

\section{Planejamento probabilístico}

Nesse capítulo são apresentados os modelos comumente utilizados em planejamento probabilístico, com uma atenção especial para modelos orientados à meta e algoritmos clássicos para esses problemas.

\subsection{Modelos para planejamento probabilístico}

O planejamento probabilístico é uma sub-área da inteligência artificial que lida com a tomada de decisão em ambientes estocásticos. Essa área procura definir como um agente deve agir em um ambiente em que suas ações têm efeitos estocásticos.

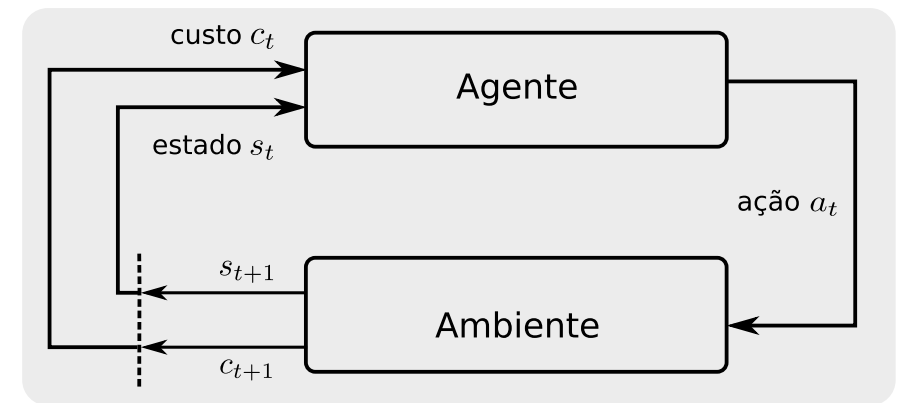

Figura 2.1: Interação entre um agente e seu ambiente. Em um estágio de interação $t$, o agente está no estado $s_{t}$ e aplica a ação $a_{t}$; em seguida ele recebe o novo estado do ambiente $s_{t+1}$ e um custo $c_{t+1}$ [Barto et al., 1995].

Problemas de planejamento probabilístico podem ser modelados como processos de decisão markovianos (Markovian Descision Processes - MDPs) [Puterman, 1994]. Um MDP modela a interação entre um agente e o seu ambiente, a Figura 2.1 ilustra como essa interação ocorre. Note que, em um estágio $t$, o agente está no estado $s_{t}$ e decide que a ação $a_{t}$ será aplicada; após aplicar a ação, ele vai para o próximo estado $s_{t+1}$ e recebe o custo $c_{t+1}$. Num MDP a função de utilidade é dada em função do custo acumulado das ações aplicadas. Assim, um MDP pode ser visto como um problema de otimização, em que o objetivo é minimizar o custo acumulado esperado da interação entre o agente e o ambiente durante um dado número de estágios, denominado horizonte. Em geral, consideramos três tipos de horizontes:

Finito: o agente atua no ambiente por um número finito predefinido de estágios;

Infinito: o agente nunca para de agir no ambiente; e

Indefinido: o agente atua no ambiente por um número indeterminado de estágios, por exemplo, até alcançar um estado meta. 


\subsubsection{Processos de decisão markovianos}

Definição 1 (Processo de Decisão Markoviano - MDP). Um MDP é definido por uma tupla $\mathcal{M}=\langle S, A, P, C, \gamma\rangle$ [Puterman, 1994], em que:

- $S$ é um conjunto de estados totalmente observáveis;

- A é um conjunto de ações; $A(s) \subseteq A$ é o conjunto de ações aplicáveis no estado $s \in S$;

- $P$ é uma função de transição probabilística, sendo que, $P\left(s^{\prime} \mid s, a\right)$ indica a probabilidade do agente ir para o estado $s^{\prime} \in S$ ao aplicar uma ação $a \in A$ no estado $s \in S$, sendo $P\left(s^{\prime} \mid s, a\right)=$ $0, \forall a \notin A(s)$;

- $C: S \times A \rightarrow \mathbb{R}$ é uma função que define o custo imediato para o agente aplicar a ação $a \in A$ em um estado $s \in S$; $e$

- $0<\gamma \leq 1$ é o fator de desconto que pondera entre custo imediato e futuro.

A solução de um MDP é uma política que define o comportamento do agente. A política ótima é aquela que minimiza o custo esperado do agente ao longo de $k$ estágios.

Definição 2 (Política). Uma política (estacionária) é uma função que mapeia estados em ações: $\pi: S \rightarrow A$. Uma política pode ser definida de forma total, isto é $\forall s \in S$, ou parcial, isto é $\forall s \in S^{\prime} \subset S$. $\triangle$

Um histórico $h$ é uma sequência de estados pelos quais o agente passa em $k$ estágios, seguindo uma política $\pi$ qualquer. Denotamos por $\mathcal{H}^{k}$ o conjunto de todos os históricos possíveis de $k$ estágios de um MDP $\mathcal{M}$. Dado um histórico $h=\left(s_{0}, s_{1}, \cdots, s_{k}\right) \in \mathcal{H}^{k}$, a probabilidade do histórico $h$ ocorrer seguindo a política $\pi$ é dada por:

$$
P(h \mid \pi)=\prod_{i=0}^{k-1} P\left(s_{i+1} \mid s_{i}, \pi\left(s_{i}\right)\right),
$$

sendo $s_{i}$ e $s_{i+1}$ os estados de $h$ visitados nos estágios $i$ e $i+1$, respectivamente; e $\pi\left(s_{i}\right)$ a ação executada no estágio $i$.

Definição 3 (Custo acumulado esperado descontado de uma política). Seja o custo acumulado descontado de um histórico $h=\left(s_{0}, s_{1}, \cdots, s_{k}\right)$ induzido por uma política $\pi$ dado por:

$$
V^{\pi}(h)=\sum_{i=0}^{k-1} \gamma^{i} C\left(s_{i}, \pi\left(s_{i}\right)\right)
$$

o custo acumulado esperado descontado de uma política $\pi$ em $k$ estágios é definido como:

$$
J(\pi, k)=\sum_{h \in \mathcal{H}^{k}} V^{\pi}(h) P(h \mid \pi) .
$$

Também podemos definir o custo acumulado esperado descontado de um estado $s$ seguindo uma política $\pi$ por $k$ estágios futuros, em função dos custos acumulados do conjunto de históricos de $k$ estágios que iniciam em $s$, denotado por $\mathcal{H}_{s}^{k}$, ou seja:

$$
V^{\pi}(s, k)=J_{s}(\pi, k)=\sum_{h \in \mathcal{H}_{s}^{k}} V^{\pi}(h) P(h \mid \pi) .
$$

Uma definição recursiva de $V^{\pi}(s, k)$ pode ser dada em função do valor dos estados sucessores $s^{\prime}$, isto é $V^{\pi}\left(s^{\prime}, k-1\right)$, como sendo a soma do custo imediato de se aplicar $\pi$ no estado $s, C(s, \pi(s))$, 
e o custo descontado dos possíveis estados visitados no futuro em $k-1$ estágios futuros, isto é, $\gamma \sum_{s^{\prime} \in S} P\left(s^{\prime} \mid s, \pi(s)\right) V^{\pi}\left(s^{\prime}, k-1\right)$, ou seja:

$$
V^{\pi}(s, k)=C(s, \pi(s))+\gamma \sum_{s^{\prime} \in S} P\left(s^{\prime} \mid s, \pi(s)\right) V^{\pi}\left(s^{\prime}, k-1\right) .
$$

Note que para um MDP de horizonte finito, $V^{\pi}(s, k)$ é um valor finito, mesmo para $\gamma=1$. Num MDP de horizonte infinito $(k=\infty), V^{\pi}(s, \infty)$ pode resultar num valor infinito. Nesse caso, o uso de $\gamma<1$ garante um valor finito.

\subsubsection{MDP de horizonte infinito}

Uma propriedade importante de um MDP de horizonte infinito é que o valor do estado $s$ seguindo uma política $\pi$ não depende do número de estágios futuros, o que faz com que a Equação 2.5 resulte em [Puterman, 1994]:

$$
V^{\pi}(s)=C(s, \pi(s))+\gamma \sum_{s^{\prime} \in S} P\left(s^{\prime} \mid s, \pi(s)\right) V^{\pi}\left(s^{\prime}\right) .
$$

O valor ótimo de um estado $s$, denotado por $V^{*}(s)$, em um horizonte infinito de ações, é aquele que minimiza o custo esperado descontado. A função valor ótima $V^{*}(s)$ para um MDP de horizonte infinito é chamada de Equação de Bellman [Puterman, 1994]:

$$
V^{*}(s)=\min _{a \in A}\left\{C(s, a)+\gamma \sum_{s^{\prime} \in S} P\left(s^{\prime} \mid s, a\right) V^{*}\left(s^{\prime}\right)\right\} .
$$

ou seja $V^{*}(s)$ é a função que minimiza o custo de se aplicar a ação ótima em $s$, somado aos valores ótimos esperados dos possíveis estados futuros.

Analogamente, num MDP de horizonte infinito, o custo acumulado esperado descontado de uma política não depende do número de estágios futuros, logo a Equação 2.3 pode ser simplificada:

$$
J(\pi)=\sum_{h \in \mathcal{H}^{\infty}} V^{\pi}(h) P(h \mid \pi) .
$$

Considerando apenas o conjunto de históricos $\mathcal{H}_{s}^{\infty}$ que iniciam em um estado $s$, o valor de uma política $\pi$ a partir de $s$ é:

$$
J_{s}(\pi)=\sum_{h \in \mathcal{H}_{s}^{\infty}} V^{\pi}(h) P(h \mid \pi) .
$$

Uma solução para um MDP de horizonte infinito pode ser encontrada pelo algoritmo de programação dinâmica Iteração de Valor (Value Iteration - VI), que usa a Equação de Bellman como uma função de atualização [Bellman, 1957]:

$$
V_{t+1}(s) \leftarrow \min _{a \in A}\left\{C(s, a)+\gamma \sum_{s^{\prime} \in S} P\left(s^{\prime} \mid s, a\right) V_{t}\left(s^{\prime}\right)\right\}, \forall s \in S .
$$

O algoritmo VI inicializa $V_{0}(s)$ com um valor qualquer e iterativamente atualiza a função valor de cada estado até a convergência, isto é, até que o erro residual de todos os estados seja menor que um erro mínimo $\epsilon$. O erro residual de um estado $s, \operatorname{Res}(s)$, na iteração $t$ é definido como:

$$
\operatorname{Res}(s)=\left|V_{t}(s)-V_{t-1}(s)\right| .
$$

Assim, o algoritmo vi termina quando $\forall s \in S, \operatorname{Res}(s)<\epsilon$. Conforme demonstrado por Puterman [1994], a função valor $V_{t}$ converge para a função valor ótima, $V^{*}$ (Equação 2.6).

Definição 4 (Política gulosa). Dada uma função valor qualquer $V: S \rightarrow \mathbb{R}$, uma política gulosa 
$\pi^{V}$ atribui para cada estado $s \in S$ uma ação gulosa que minimiza $V(s)$ :

$$
\pi^{V}(s)=\underset{a \in A}{\arg \min }\left\{C(s, a)+\sum_{s^{\prime} \in S} P\left(s^{\prime} \mid s, a\right) V\left(s^{\prime}\right)\right\} .
$$

Dessa forma, com a função valor ótima, $V^{*}$ (Equação 2.6), obtida após a convergência do algoritmo VI, é possível definir a política ótima, denotada por $\pi^{*}$, como a política gulosa associada à $V^{*}(s)$, isto é, $\pi^{*}(s)=\pi^{V^{*}}(s)$.

Definição 5 (Política ótima para um MDP de horizonte infinito). A política ótima, denotada por $\pi^{*}$, é a política que minimiza o custo acumulado esperado descontado a partir de todos os estados, isto é, $\forall \pi: J_{s}\left(\pi^{*}\right) \leq J_{s}(\pi), \forall s \in S$.

\subsubsection{Planejamento orientado à meta}

Em geral, problemas de planejamento envolvem um possível estado inicial $s_{0}$ e uma meta $\mathbb{G}$, dada por um conjunto de proposições que devem ser satisfeitas no estado meta. Assim, o objetivo de um problema de planejamento probabilístico passa a ser a escolha de ações que minimizem o custo acumulado esperado de levar o agente do estado inicial para um estado meta. Tais problemas possuem um horizonte indefinido, uma vez que o agente para de atuar ao alcançar um estado meta. Neste trabalho, especificaremos tais problemas através de MDPs orientados à meta (Goal Oriented MDPs - GMDPs). Um GMDP é um MDP com um possível estado inicial $s_{0}$ (ou um conjunto de estados iniciais) e um conjunto de possíveis estados meta.

Definição 6 (MDP orientado à meta (Goal Oriented MDP - GMDP)). Um GMDP é uma tupla $\mathcal{M}=\left\langle S, A, P, C, s_{0}, G\right\rangle$ [Teichteil-Königsbuch, 2012], em que $S, A, P$ e $C$ são definidos como em um MDP (Definição 1), e:

- $s_{0} \in S$ é o estado inicial ${ }^{1}, e$

- $G \subseteq S$ é o conjunto de estados meta absorventes e sem custo, isto é,

$$
\forall(s, a) \in G \times A: P(s \mid s, a)=1 \text { e } C(s, a)=0 .
$$

Note que, apesar de existirem ações aplicáveis nos estados meta $s \in G$, essas ações são absorventes com custo nulo, o que faz com que o agente permaneça na meta. Modelar estados meta como estados absorventes é apropriado para o uso dos algoritmos de programação dinâmica, baseados em VI, como veremos nas próximas seções.

A solução para um GMDP é uma política (possivelmente parcial) que satisfaz algum critério de otimalidade, por exemplo, a política de menor custo esperado que alcança um estado meta a partir do estado inicial $s_{0}$.

Como discutimos no Capítulo 1, um GMDP, bem como um MDP, pode ser representado por um grafo direcionado em que os nós são rotulados por estados e as arestas por ações. A Figura 2.2 mostra exemplos de grafos que representam GMDPs em que $s_{0}$ é o estado inicial e $s_{g}$ é o estado meta. Note que a única ação aplicável em $s_{g}$ possui um custo nulo e leva somente para $s_{g}$ (estado meta absorvente). Note ainda que um estado absorvente que não é um estado meta é um beco-sem-saída (estado $s_{3}$ da Figura 2.2(b)). (Por simplicidade chamaremos esses grafos de grafos.)

O grafo guloso $G_{V}$ de um GMDP é o grafo dos estados alcançáveis a partir do estado inicial $s_{0}$ executando uma política gulosa $\pi^{V}$ (também denotado por $G_{\pi}$ ). Assim, $s_{0}$ é o nó raiz de $G_{V}$, e os filhos de cada estado $s \in G_{V}$ são os nós que podem ser obtidos ao executar a ação $\pi^{V}(s)$. A Figura 2.3 mostra um exemplo de um grafo guloso de um GMDP (subgrafo com nós em cinza) para a política gulosa $\pi^{V}=\left\{s_{0}: a_{0} ; s_{1}: a_{0} ; s_{2}: a_{0} ; s_{4}: a_{0} ; s_{g}: a_{2}\right\}$ aplicada a partir do estado inicial.

\footnotetext{
${ }^{1}$ Também seria possível definir um conjunto de estados iniciais $S_{0} \subseteq S$. No entanto, definimos GMDPs com apenas
} 


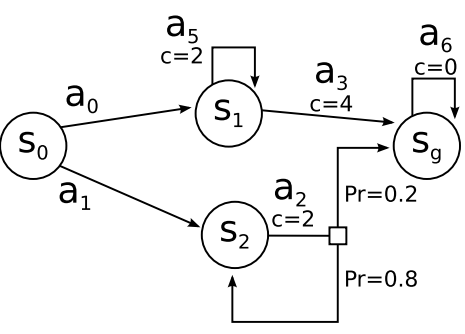

(a)

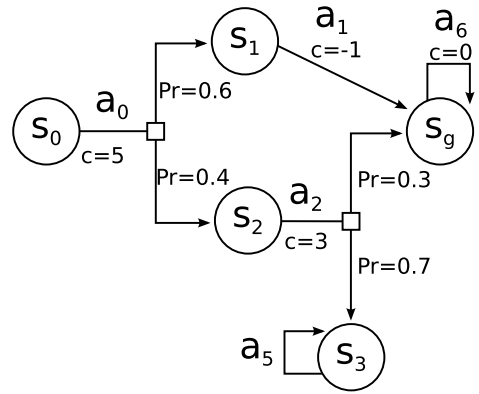

(b)

Figura 2.2: Exemplos de GMDPs representados como grafos de transição de estados. Nós são rotulados por estados $s_{i}$; arestas são rotuladas por ações; transições determinísticas são arestas orientadas com probabilidade 1; transições probabilísticas são indicadas por arestas que se ramificam a partir de uma caixa $\square$, cada ramo com $\mathrm{Pr}<1$. (Omitimos custos unitários de ações.)

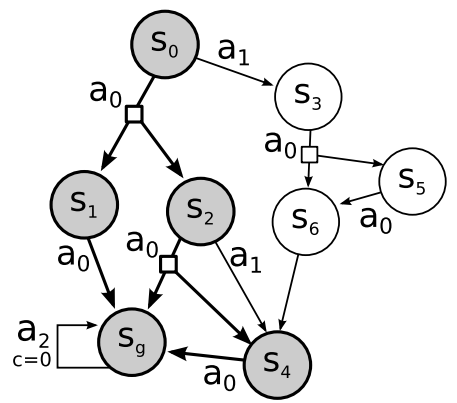

Figura 2.3: Exemplo de grafo guloso $G_{V}$ em um GMDP para a política gulosa $\pi^{V}$, a partir de $s_{0}$. O grafo guloso $G_{V}$ é composto pelos estados em cinza e as arestas escuras. Note que $s_{3}, s_{5}$ e $s_{6}$ não fazem parte de $G_{V}$, pois a política $\pi^{V}$ nunca leva o agente para esses estados.

Note que, um GMDP descreve apenas o modelo do mundo (um sistema de transição de estados). Assim, ainda é necessário definir um critério de otimização para problemas modelados por um GMDP, que definirá qual é a melhor solução para o problema. Para isso, é preciso fazer algumas suposições, por exemplo: é possivel alcançar um estado meta a partir de todos os estados $s \in S$; é possível alcançar um estado meta a partir de $s_{0}$; políticas que não alcançam um estado meta têm um custo esperado infinito; etc. Diferentes suposições e critérios de otimização definem diferentes subclasses de GMDPs, como veremos nos modelos introduzidos na próxima seção e no próximo capítulo.

\subsubsection{MDP de caminho estocástico mínimo}

Definição 7 (MDP de Caminho Estocástico Mínimo (Stochastic Shortest Path MDP SSP MDP)). Um SSP MDP [Bertsekas e Tsitsiklis, 1991] é um GMDP $\mathcal{M}=\left\langle S, A, P, C, s_{0}, G\right\rangle$ que faz as seguintes suposições:

(S1) é possivel alcançar a meta a partir de todos os estados de $S$; e

(S2) toda política que não leva para um estado meta tem um custo esperado infinito.

Num SSP MDP, uma vez que o agente para de agir ao encontrar um estado meta, o horizonte é indefinido e, por isso, o custo não precisa ser descontado para a convergência da função de atualização de Bellman (Equação 2.9). Assim, em um SSP MDP o objetivo é encontrar uma política

um possível estado inicial, sem perda de generalidade. Seja $P_{0}(s)$ a probabilidade de $s \in S_{0}$ ser o estado inicial; poderíamos criar um novo GMDP em que $s_{0}$ é um estado inicial auxiliar com uma única ação aplicável $a_{0}$ que leva para $s \in S_{0}$ com probabilidade $P_{0}(s)$. 
que minimize o custo esperado acumulado (sem desconto) de levar o agente do estado inicial para um estado meta.

Para formalizarmos as suposições do SSP MDP, e o seu critério de otimização, definimos primeiro a probabilidade de alcançar um estado meta a partir de um estado $s$ qualquer e o custo esperado dos históricos que alcançam a meta a partir desse estado.

Seja $S^{\prime} \subseteq S$ um conjunto qualquer de estados e $\mathcal{H}_{s}^{S^{\prime}}$ o conjunto de históricos que partem do estado $s \in S$ e alcançam um estado $s^{\prime} \in S^{\prime}$. Definimos a probabilidade do agente alcançar um estado $s^{\prime} \in S^{\prime}$ a partir de um estado $s \in S$, seguindo uma política $\pi$, como a soma das probabilidades dos históricos de $\mathcal{H}_{s}^{S^{\prime}}$ [Mausam e Kolobov, 2012]:

$$
P_{\pi}^{S^{\prime}}(s)=\sum_{h \in \mathcal{H}_{s}^{S^{\prime}}} P(h \mid \pi)
$$

sendo $P(h \mid \pi)$ a probabilidade do histórico $h$ ocorrer seguindo a política $\pi$ (Equação 2.1). Também podemos definir o custo esperado dos históricos que alcançam um estado em $S^{\prime} \subseteq S$ a partir de $s$ seguindo uma política $\pi$, denotado por $C_{\pi}^{S^{\prime}}(s)$, como a soma dos custos dos históricos de $h \in \mathcal{H}_{s}^{S^{\prime}}$ (Equação 2.2) ponderados por suas probabilidades (Equação 2.1), isto é:

$$
C_{\pi}^{S^{\prime}}(s)=\sum_{h \in \mathcal{H} S_{s}^{\prime}} V^{\pi}(h) P(h \mid \pi) .
$$

Dizemos que uma política $\pi$ é $s$-própria se ela tem probabilidade 1 de finalmente levar o agente para um estado meta partindo do estado $s \in S$, isto é, se $P_{\pi}^{G}(s)=1$ (Equação 2.10).

Proposição 1. Dado um estado $s \in S$, se uma política $\pi$ é s-própria, então, para todo estado $s^{\prime}$ alcançável a partir de s seguindo $\pi$ (conjunto denotado por Alc $\left.(s, \pi)^{2}\right), \pi$ também é $s^{\prime}$-própria. Isto é, se $P_{\pi}^{G}(s)=1$, então $\forall s^{\prime} \in \operatorname{Alc}(s, \pi): P_{\pi}^{G}\left(s^{\prime}\right)=1$.

Política própria. Uma política $\pi$ é própria se para todo estado $s \in S, \pi$ é $s$-própria. Em outras palavras, $\pi$ é própria se ela tem probabilidade 1 de finalmente levar o agente para um estado meta a partir de todos os estados, isto é, $\forall s \in S: P_{\pi}^{G}(s)=1$ (Equação 2.10). Uma política $\pi$ é dita imprópria se não for própria, ou seja, quando existe ao menos um estado com probabilidade menor que 1 de levar o agente para um estado meta, ou seja $\exists s \in S: P_{\pi}^{G}(s)<1$.

A Figura 2.4 mostra um exemplo de GMDP com uma política própria $\pi_{1}=\left\{s_{0}: a_{0} ; s_{1}: a_{2}\right\}$ (omitimos ações atribuídas ao estado meta por simplicidade) e políticas impróprias $\pi_{2}, \pi_{3}$ e $\pi_{4}$. Note que, seguindo a política $\pi_{1}$, o agente sempre atingirá a meta, isto é $P_{\pi_{1}}^{G}\left(s_{0}\right)=1$. Por outro lado, a política $\pi_{2}=\left\{s_{0}: a_{0} ; s_{1}: a_{1}\right\}$ tem uma probabilidade de 0.99 de levar o agente para o estado meta $s_{g}$, isto é $P_{\pi_{2}}^{G}\left(s_{0}\right)=0.99$; e probabilidade 0.01 de levar o agente para o estado $s_{1}$ e permanecer nele. Portanto, $\pi_{2}$ é uma política imprópria. As políticas $\pi_{3}=\left\{s_{0}: a_{1} ; s_{1}: a_{1}\right\}$ e $\pi_{4}=\left\{s_{0}: a_{1} ; s_{1}: a_{2}\right\}$ deixam o agente preso no estado $s_{0}$ aplicando a ação $a_{1}, \operatorname{logo} P_{\pi_{3}}^{G}\left(s_{0}\right)=P_{\pi_{4}}^{G}\left(s_{0}\right)=0$ e assim estas políticas também são impróprias. Observamos aqui que uma política imprópria, pode ser $s$-própria para algum estado $s \in S$. Por exemplo, $\pi_{4}$ é imprópria, pois $P_{\pi_{4}}^{G}\left(s_{0}\right)=0$, e é $s_{1}$-própria, pois $P_{\pi_{4}}^{G}\left(s_{1}\right)=1$.

Políticas próprias em algum momento alcançam um estado meta e, portanto, têm um custo esperado finito. Considerando que a função custo seja positiva, então políticas impróprias terão um custo esperado infinito. Porém, para uma função custo qualquer, o custo esperado de uma política pode ser um valor real finito, ou até mesmo infinito negativo.

Podemos agora redefinir formalmente as suposições S1 e S2 dos SSP MDPs:

(S1) $\exists \pi: P_{\pi}^{G}(s)=1, \forall s \in S$, isto é, existe ao menos uma política própria, e

(S2) $\forall \pi$ : , se $\exists s \in S \mid P_{\pi}^{G}(s)<1$ então $J(\pi)=\infty$, isto é, toda política imprópria tem custo esperado infinito, sendo $J(\pi)$ dado pelas equações 2.7 e $2.2 \operatorname{com} \gamma=1$.

\footnotetext{
${ }^{2}$ Também chamamos o conjunto de estados alcançáveis a partir de um estado $s$ pela política $\pi$ de fecho transitivo de $P \operatorname{com} \pi$ a partir de $s$ [Ghallab et al., 2016].
} 


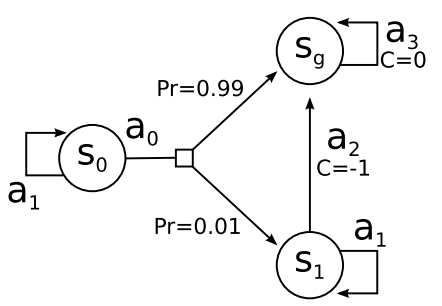

Figura 2.4: Exemplo de GMDP com política própria $\left(\pi_{1}=\left\{s_{0}: a_{0} ; s_{1}: a_{2}\right\}\right)$ e políticas impróprias $\left(\pi_{2}=\left\{s_{0}: a_{0} ; s_{1}: a_{1}\right\}, \pi_{3}=\left\{s_{0}: a_{1} ; s_{1}: a\right\}\right.$ e $\left.\pi_{4}=\left\{s_{0}: a_{1} ; s_{1}: a_{2}\right\}\right)$.

O GMDP da Figura 2.4 é um exemplo de SSP MDP. A política $\pi_{1}=\left\{s_{0}: a_{0} ; s_{1}: a_{2}\right\}$ é uma política própria o que satisfaz a suposição S1. Como todas as ações têm custo positivo, notamos que a suposição S2 também é satisfeita, uma vez que para as políticas impróprias $\pi_{2}, \pi_{3}$ e $\pi_{4}$, o custo esperado é $\infty$. Note que a ação $a_{2}$ tem custo negativo, e mesmo assim as suposições do SSP MDP são satisfeitas.

Política ótima. A política ótima de um SSP MDP $\mathcal{M}$ é uma política própria que minimiza o custo esperado dos históricos que alcançam um estado meta (Equação 2.11), conforme a seguir:

$$
\pi^{*}=\underset{\pi \text { é própria }}{\arg \min } C_{\pi}^{G}(s), \forall s \in S .
$$

Como na solução de um MDP de horizonte infinito, a política ótima $\pi^{*}$ de um SSP MDP é a política gulosa associada à função $V^{*}(s)=\min _{\pi} V^{\pi}(s)$, com $\gamma=1$, que satisfaz a equação de otimalidade de Bellman [Bertsekas e Tsitsiklis, 1991]:

$$
V^{*}(s)= \begin{cases}0 & \text { se } s \in G, \\ \min _{a \in A(s)}\left\{C(s, a)+\sum_{s^{\prime} \in S} P\left(s^{\prime} \mid s, a\right) V^{*}\left(s^{\prime}\right)\right\} & \text { caso contrário. }\end{cases}
$$

Um meio de computar a solução de um SSP MDP é calcular a função $V^{*}$ usando programação dinâmica. O algoritmo VI pode resolver um SSP MDP utilizando a Equação 2.13 como uma função de atribuição para a função valor:

$$
V^{t+1}(s) \leftarrow \min _{a \in A(s)}\left\{C(s, a)+\sum_{s^{\prime} \in S} P\left(s^{\prime} \mid s, a\right) V^{t}\left(s^{\prime}\right)\right\},
$$

sendo $V^{0}(s)$ um valor qualquer $\forall s \notin G$ e $V^{0}(s)=0, \forall s \in G$.

O algoritmo VI atualiza a função valor de todos os estados a cada iteração e por isso é chamado de algoritmo de programação dinâmica síncrono. Após a convergência, esse algoritmo cria uma política total, isto é, uma política definida para todos os estados de $S$. No entanto, dado um GMDP, em que o estado inicial $s_{0}$ é conhecido, só é necessário definir a política para os estados alcançáveis a partir de $s_{0}$. Essa é uma característica importante, uma vez que o tamanho do espaço de estados cresce exponencialmente em função do número de proposições atômicas $\mathbb{P}$, isto é, $|S|=2^{|\mathbb{P}|}$, o que torna inviável manter todo o espaço de estados na memória. Na seção a seguir, são descritos algoritmos assincronos que atualizam apenas um subconjunto de estados a cada iteração.

\subsection{Algoritmos assíncronos de planejamento probabilístico}

Na seção anterior foi descrita uma abordagem síncrona para resolução de SSP MDPs, o algoritmo VI, em que o passo de atualização é realizado para todos os estados a cada iteração. Nessa seção descrevemos um esquema mais eficiente para resolver SSP MDPs, que só atualiza um estado a cada iteração. Por fim, apresentamos dois algoritmos que implementam esse esquema. 
O esquema de encontrar e revisar (Find and Revise - F\&R) define um meio geral de resolver SSP MDPs de forma assíncrona [Bonet e Geffner, 2003a]. Esse esquema se baseia nas suposições S1 e S2 dos SSP MDPs para garantir que uma política ótima seja encontrada. Para isso, o esquema F\&R propõe que apenas um estado seja atualizado a cada iteração e usa uma heurística admissível para inicializar a função valor, reduzindo o tempo de convergência do algoritmo. Esse esquema converge quando todos os estados do grafo guloso enraizado no estado inicial tiverem um erro residual menor que $\epsilon$. Bonet e Geffner [2003a] mostraram que esse esquema converge caso a heurística utilizada seja admissível.

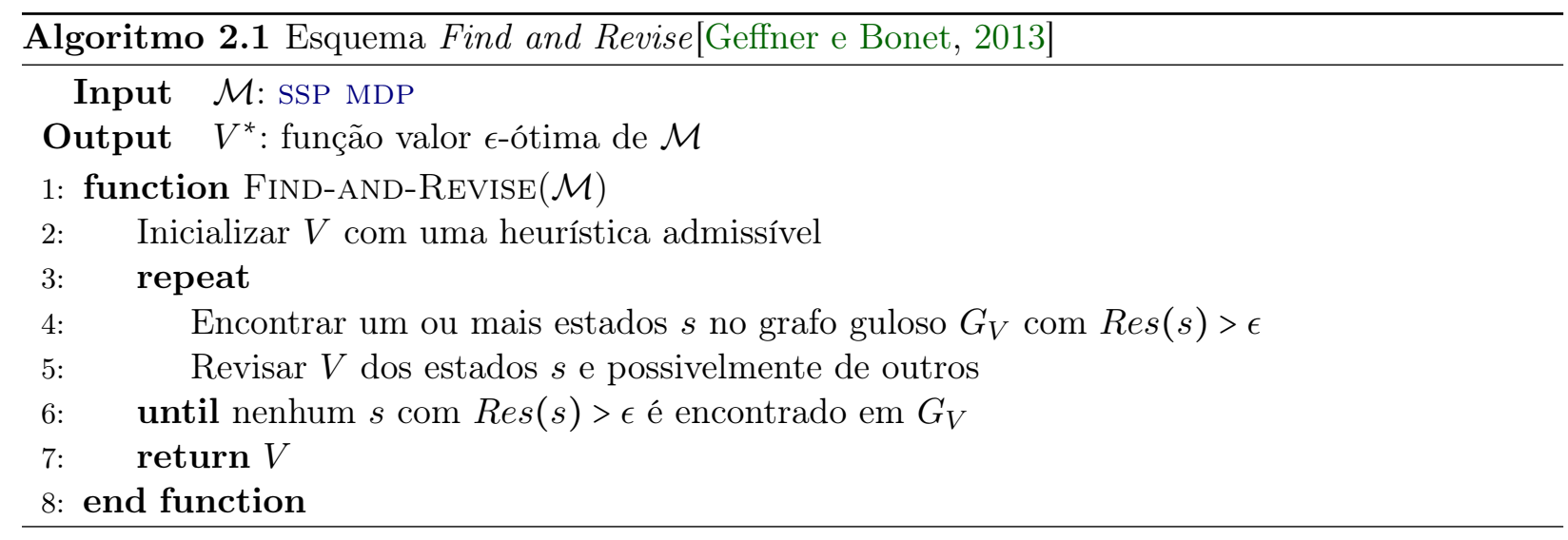

Dado um SSP MDP $\mathcal{M}$, o esquema F\&R usa o grafo guloso $G_{V}$, enraizado em $s_{0}$, para encontrar estados que ainda não convergiram e precisam ser atualizados, computando assim a função valor apenas para os estados alcançáveis a partir de $s_{0}$, gerando uma política parcial definida apenas para um conjunto $S^{\prime} \subseteq S$, com $S^{\prime}=A l c\left(s_{0} . \pi\right)$, sendo $\pi$ a política gulosa.

Assim como o VI, o F\&R calcula a função valor $V^{*}$ com erro residual máximo $\epsilon$. O Algoritmo 2.1 mostra uma descrição de alto nível do esquema $F \& R$. Na linha 2 a função valor é inicializada utilizando uma heurística admissível. O esquema F\&R procura no grafo guloso $G_{V}$ por estados com erro residual maior que $\epsilon$ (Linha 4), revisando-os, isto é, atualizando a função valor desses estados (Linha 5), até que não haja nenhum estado com erro residual maior que $\epsilon$ no grafo guloso $G_{V}$ (Linha $6)$.

A eficiência dos algoritmos que implementam o esquema $F \& R$ depende da ordem em que os estados são atualizados e, principalmente, da heurística que é utilizada para inicializar a função valor. Quanto mais o valor da heurística de um estado $s, V^{0}(s)$, se aproxima do valor ótimo, $V^{*}(s)$, menor o número de atualizações necessárias para que esse estado convirja para o valor ótimo. Os algoritmos apresentados nas próximas seções implementam o esquema F\&R, utilizando estratégias diferentes para a escolha do estado que será atualizado. No Capítulo 4 discutimos diferentes tipos de heurísticas usadas para a inicialização da função valor $V(s)$.

\subsubsection{Os algoritmos RTDP e LRTDP}

O algoritmo de programação dinâmica em tempo real (Real-Time Dynamic Programming RTDP) [Barto et al., 1995], é um algoritmo de planejamento probabilístico que, a partir do estado inicial, seleciona os estados a serem atualizados através de amostragens, chamadas de trials. Um trial simula a execução de uma política partindo do estado inicial. A Figura 2.5 ilustra como essa simulação é feita. Dado um estado $s$, o agente seleciona uma ação gulosa $a$ e sorteia um de seus estados sucessores $s^{\prime}$, de acordo com a probabilidade $P\left(s^{\prime} \mid s, a\right)$. Essa operação é repetida até que um estado meta seja encontrado. O RTDP atualiza, a cada iteração, a função valor de um único estado utilizando a Equação de Bellman sem desconto (Equação 2.14), de forma similar ao VI.

O algoritmo RTDP rotulado (Labeled RTDP - LRTDP) [Bonet e Geffner, 2003b] é uma extensão do RTDP que segue o esquema $\mathrm{F} \& \mathrm{R}$. Esse algoritmo rotula os estados que já convergiram como resolvidos (solved) e para de atualizar a função valor desses estados, acelerando assim a convergência do algoritmo. 


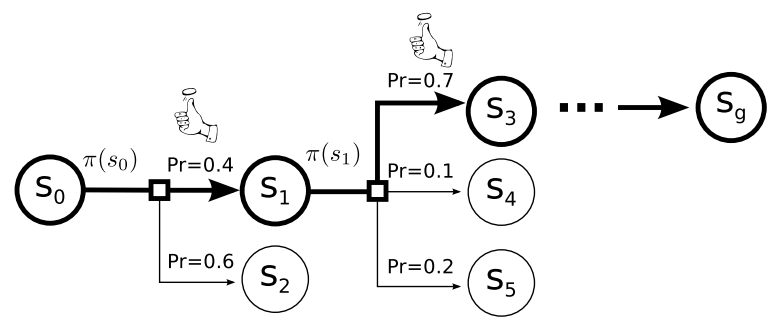

Figura 2.5: Exemplo de trial do algoritmo RTDP. Linhas escuras indicam os estados visitados pela simulação. Uma simulação termina em um estado meta $s_{g} \in G$.

No Algoritmo 2.2, o procedimento LTRDP recebe um estado inicial $s_{0}$ e um erro máximo $\epsilon$ e realiza trials até que $s_{0}$ tenha sido rotulado como solved, o que significa que a função valor de todos os estados do grafo guloso convergiu. O procedimento LTRDP-TRIAL do Algoritmo 2.2 executa um trial: ele recebe um estado e seleciona uma ação gulosa para ser executada (Linha 13); atualiza a função valor do estado visitado (Linha 14); e em seguida sorteia o próximo estado a ser visitado simulando uma interação com o ambiente através do método NEXT-STATE (Linha 15). Um trial continua visitando estados até que um estado meta, ou um estado rotulado como solved, seja visitado. Se um estado visitado $s$ ainda não possui um valor definido, o LRTDP chama o método INITIALIZE, que usa a heurística $h$ para inicializar a função valor de $s$. Note que cada estado visitado é armazenado na pilha visited (Linha 9). Ao final de cada trial (Linhas 17-22) é feita uma chamada ao método CHECK-SOLVED (Linha 19) para cada estado armazenado em visited (em ordem inversa à que foram empilhados) para verificar se esse estado convergiu e, portanto, pode ser rotulado como solved. O método CHECK-SOLVED verifica se todos os estados do grafo guloso enraizado no estado $s_{i}$ já convergiram e, nesse caso, rotula $s_{i}$ como solved. Se o estado $s_{i}$ não for rotulado, o algoritmo não analisa os demais estados do trial (Linha 20). A ideia é que o estado $s_{i}$ faz parte do grafo guloso dos estados visitados anteriormente no trial (os antecessores de $s_{i}$ ) e se $s_{i}$ não convergiu, seus antecessores também não convergiram.

À medida que os estados são rotulados, o tamanho dos trials diminui. Com isso, estados não resolvidos serão visitados com maior frequência, o que reduz o tempo de convergência do algoritmo LRTDP, comparado ao RTDP. A forma como os trials são realizados, ponderando o sorteio do estado sucessor pela probabilidade de transição da ação gulosa faz com que o LRTDP visite mais vezes estados com maior probabilidade de serem alcançados, levando estes estados a convergirem mais rapidamente. Isso reforça a característica anytime desse algoritmo: a qualquer momento é possível extrair uma política útil.

\subsection{Outros algoritmos eficientes para MDPs}

ILAO* . Outro algoritmo assíncrono é o Loop AO* (LAO* ) [Hansen e Zilberstein, 2001], uma generalização do algoritmo de busca heurística $\operatorname{AND} / \mathrm{OR}\left(\mathrm{AO}^{*}\right)$, que garante encontrar soluções em problemas com laços. Algumas alterações no algoritmo LAO* permitem uma implementação mais eficiente, chamada Improved LAO* (ILAO*). No Algoritmo 2.3, vemos que para escolher os nós que serão atualizados, o algoritmo ILAO* expande todos os nós do grafo guloso armazenando-os em uma pilha (Linha 4). Em seguida, atualiza o valor de cada estado visitado na expansão do grafo seguindo a ordem inversa em que foram empilhados (Linhas 5-11). Assim como o LRTDP, o ILAO* usa o método InITIALIZE para inicializar a função valor de estados que nunca foram visitados. Esse procedimento é repetido até que o maior erro residual dos estados do grafo guloso $G_{V}$ seja menor que $\epsilon$ (Linha 12).

Uma das vantagens deste algoritmo sobre o (L)RTDP é que ele analisa todos os estados que podem ser alcançados a partir de uma mesma ação numa mesma iteração. Isso pode reduzir o tempo de convergência do algoritmo, uma vez que, a cada iteração, ele atualiza um número maior de estados. 


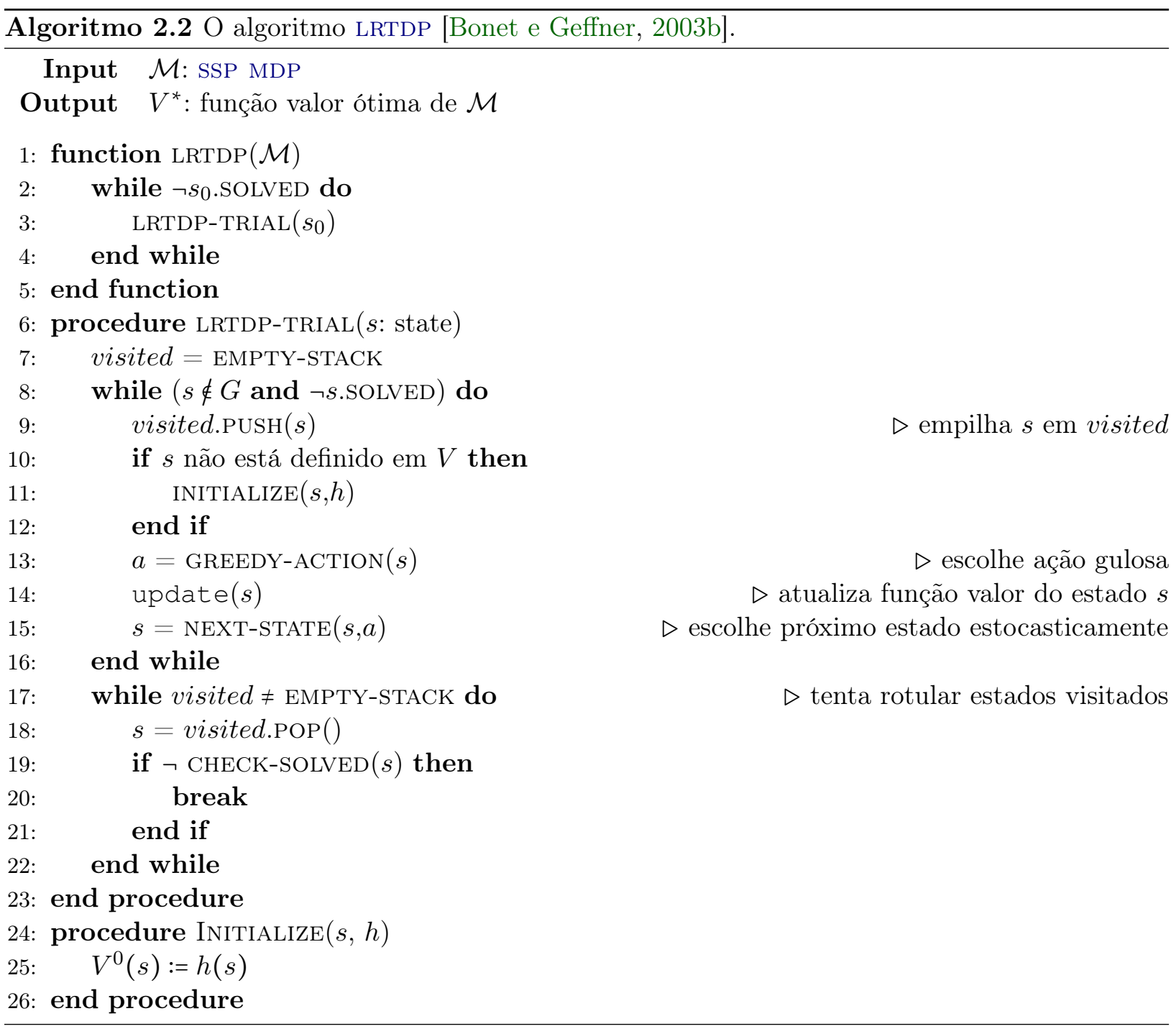

TVI. O algoritmo de iteração de valor topológico (Topological Value Iteration - TVI) [Dai e Goldsmith, 2007] é um algoritmo de programação dinâmica assíncrona que atualiza um subconjunto de estados a cada iteração. TVI particiona o espaço de estados criando uma árvore na qual cada vértice corresponde a uma componente fortemente conexa (Strongly Connected Component - SCC) do grafo de transição de estados. Uma SCC é um sub-grafo conexo máximo, isto é, cada SCC contém apenas pares de estados alcançáveis a partir um do outro. TVI computa a função valor ótima de todos os estados de uma componente de cada vez, seguindo a ordem topológica reversa da árvore de SCCs.

SPUDD. Até o momento, definimos MDPs com uma representação explícita dos estados. Também podemos representar os estados de forma fatorada, através de um conjunto de variáveis de estado $\mathcal{X}$ que caracterizam o mundo [Boutilier et al., 1999], isto é, o estado $s$ é representado por um vetor de valorações, $s=\vec{x}$. As funções custo e de transição também podem ser descritas de maneira fatorada. Assim, ao invés de definirmos a probabilidade de transitar de um estado $s$ para um estado $s^{\prime}$ ao aplicar uma ação $a$, podemos definir qual a probabilidade de cada variável $x^{\prime} \in \mathcal{X}$ assumir um certo valor, dado o estado $\vec{x}$ no instante atual e a ação $a$ aplicada. Diagramas de decisão binária (BDDs) [Bryant, 1986] e algébricos (ADDs) [Bahar et al., 1997] podem ser utilizados para representar essas funções de forma compacta ao aglomerar estados similares e realizar as operações de atualização.

O algoritmo SPUDD [Hoey et al., 1999] é uma versão do algoritmo vi que usa programação dinâmica simbólica. Este algoritmo leva em consideração a entrada fatorada e também representa a função valor de maneira fatorada, computando $V^{*}$ sem enumerar o conjunto completo de estados $S$. Para isso, SPUdD faz a atualização de Bellman para conjuntos de estados, realizando operações 


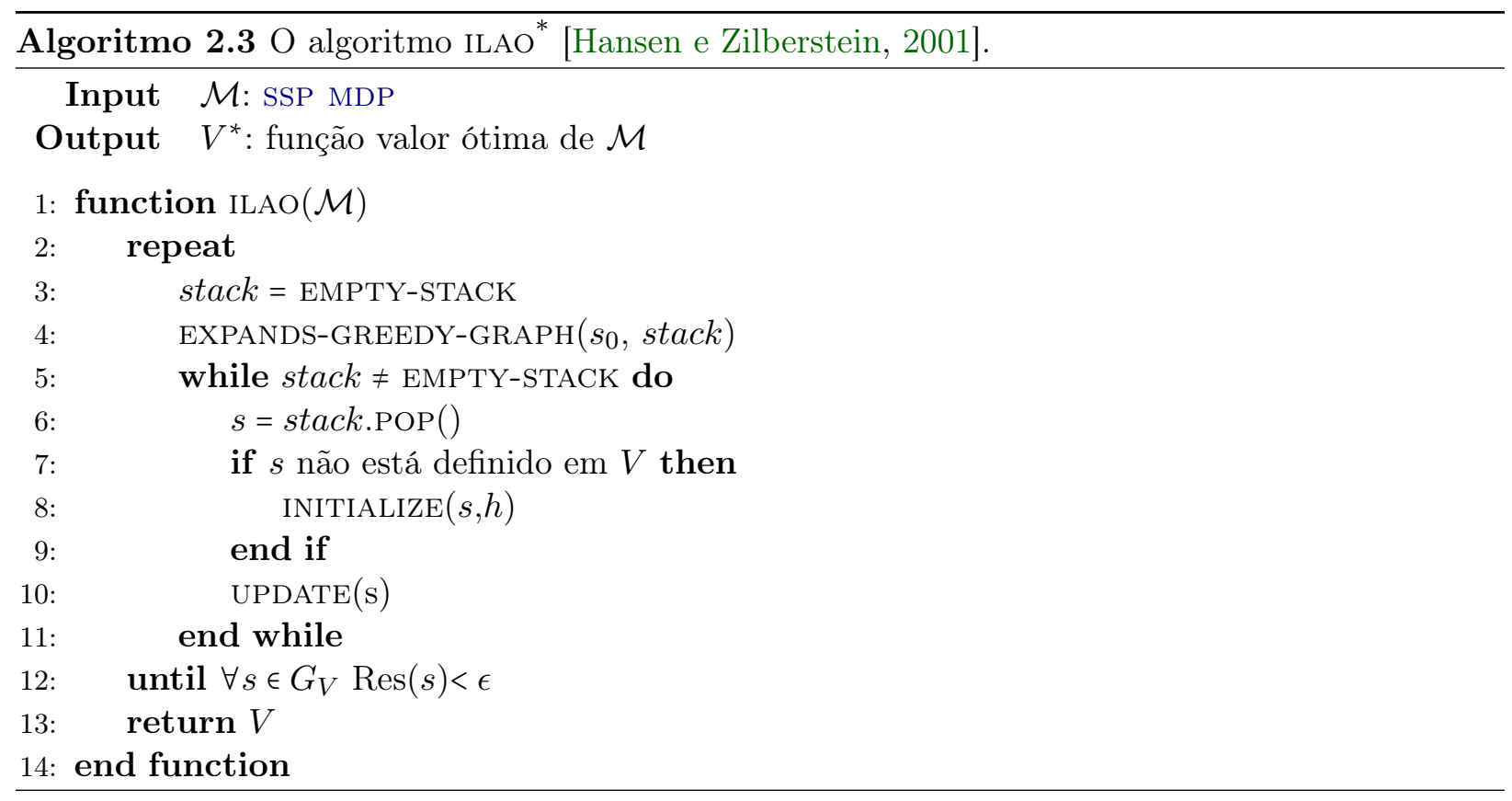

entre ADDs que representam as funções valor, custo e de transição. O sPUDD tem características de convergência iguais ao VI, pois faz a mesma computação, no entanto, ele agrupa estados estruturalmente similares, o que permite uma redução do tempo de convergência e consumo de memória.

\subsection{Representação de um GMDP a partir de uma linguagem de ações}

O uso de uma linguagem de descrição de ações permite representar problemas de planejamento de forma compacta [Fikes e Nilsson, 1971; Fox e Long, 2003; Mcdermott et al., 1998; Sanner, 2010; Younes e Littman, 2004]. Além disso, tais linguagens permitem que um agente raciocine sobre a estrutura de um problema de planejamento sem ter que enumerar todos os estados e a função de transição. Essa é a principal característica dos algoritmos de planejamento automatizado. Nesta seção, mostramos como uma linguagem de ações, baseada nas linguagens STRIPS [Fikes e Nilsson, 1971] e PDDL (Planning Domain Definition Language) [Mcdermott et al., 1998], pode ser utilizada para representar problemas de planejamento determinístico, em que não existe incerteza nos efeitos das ações, e como essa linguagem pode ser estendida para representar GMDPs e problemas de planejamento não-deterministico.

\subsubsection{Ações determinísticas}

A linguagem de ações que adotaremos especifica um problema de planejamento determinístico em função de um conjunto de proposições atômicas $\mathbb{P}$ que descrevem as propriedades do mundo. Essa linguagem define uma ação determinística $a$ pela tripla $\langle$ Precond, Add, Del $\rangle$ em que: $\operatorname{Precond}(a) \subseteq \mathbb{P}$ representa a condição de aplicabilidade de $a$ dada por um conjunto de proposições que devem ser verdadeiras no estado em que a ação $a$ será executada; $A d d(a) \subseteq \mathbb{P}$ representa as propriedades (proposições) que se tornam verdadeiras após a execução da ação $a$ (também chamadas de efeitos positivos de $a)$ e $\operatorname{Del}(a) \subseteq \mathbb{P}$ representa as propriedades que se tornam falsas após a execução da ação $a$ no estado $s$ (efeitos negativos de $a$ ). Por exemplo, a ação move de $l o c_{2}$ para $l o c_{g}$ do robô L2D2 da Figura 1.6, pode ser especificada conforme a Figura 2.6.

Definição 8 (Problema de planejamento determinístico). Um problema de planejamento deterministico é definido por uma tupla $\mathcal{P}^{\text {det }}=\left\langle\mathbb{P}, \mathbb{A}, C, s_{0}, \mathbb{G}\right\rangle$, em que:

- $\mathbb{P}$ é um conjunto de proposições atômicas que descrevem as propriedades do mundo; 


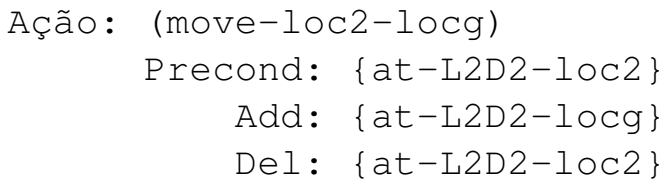

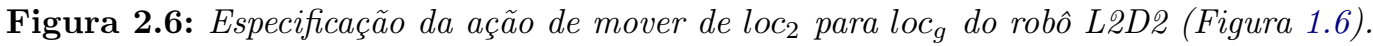

- $\mathbb{A}$ é um conjunto de ações deterministicas, cada uma descrita por uma tripla $\langle$ Precond, Add, Del $\rangle$, com Precond $\subseteq \mathbb{P}, A d d \subseteq \mathbb{P}$ e Del $\subseteq \mathbb{P}$

- $C: \mathbb{A} \rightarrow \mathbb{R}$ é uma função que define o custo de cada ação (quando omitido assumimos custo unitário para todas as ações);

- $s_{0} \subseteq \mathbb{P}$ é um conjunto de proposições verdadeiras no estado inicial; e

- $\mathbb{G} \subseteq \mathbb{P}$ é um conjunto de proposições que descrevem a meta.

Um estado é representado fazendo-se a suposição do mundo fechado, isto é, $s \subseteq \mathbb{P}$ e assumimos que uma proposição que não pertença a um estado é falsa, e que um estado $s \in S$ é representado apenas pelas proposições que são verdadeiras neste estado. Logo, uma proposição $p \in \mathbb{P}$ é verdadeira em um estado $s \in S$ se e somente se, $p \in s$ (na literatura de verificação de modelos é comum utilizar uma função de rotulagem $\mathcal{L}: S \rightarrow 2^{\mathbb{P}}$, que rotula cada estado com um conjunto de proposições, como veremos no Capítulo 5).

Definição 9 (Modelo de transição de estados). Seja $\mathcal{P}^{\text {det }}=\left\langle\mathbb{P}, \mathbb{A}, C, s_{0}, \mathbb{G}\right\rangle$ um problema de planejamento determinístico (Definição 8). O modelo de transição de estados de $\mathcal{P}^{\text {det }}$ é dado por uma tupla $\mathcal{M}\left(\mathcal{P}^{\text {det }}\right)=\left\langle S, A, T, C, s_{0}, G\right\rangle$, em que:

- $S=2^{\mathbb{P}}$ é o conjunto de estados, sendo que $2^{\mathbb{P}}$ representa o conjunto potência de $\mathbb{P}$;

- $A(s)=\{a \mid a \in \mathbb{A}$ e Precond $(a) \subseteq s\}$ é o conjunto das ações aplicáveis em $s \in S$;

- $T: S \times A \rightarrow S$ é a função de transição determinística, sendo $T(s, a)=s \backslash \operatorname{Del}(a) \cup \operatorname{Add}(a), \forall s \in$ $S, \forall a \in A(s)$;

- $s_{0} \in S$ é o estado inicial; e

- $G=\{s \in S \mid \mathbb{G} \subseteq s\}$ é o conjunto de estados meta em que todas as proposições da meta $\mathbb{G}$ são verdadeiras, isto é, $s \in G \Leftrightarrow \mathbb{G} \subseteq s$.

A solução de um problema de planejamento determinístico é um plano: uma sequência de ações, que leva o agente do estado inicial para o estado meta.

A Figura 2.7 mostra um paralelo entre as duas representações. Assim, um estado representado por um conjunto de fatos $s \subseteq \mathbb{P}$ tem um vértice equivalente do sistema de transição $s \in S$. Além disso, cada ação descrita pela linguagem de ações $a \in \mathbb{A}$ possui uma ação relacionada no sistema de estados $a \in A$.

\subsubsection{Ações probabilísticas}

Para representar o efeito probabilístico das ações é possível estender a linguagem de especificação de ações com uma lista de efeitos probabilísticos:

$$
a=\langle\text { Precond, Effects }\rangle,
$$

em que Effects é uma lista de efeitos com probabilidades associadas $\left[p_{1}:\left(A d d_{1}, D_{e} l_{1}\right), \ldots, p_{n}\right.$ : $\left.\left(A d d_{n}, D e l_{n}\right)\right]$, sendo que $\sum_{i=1}^{n} p_{i}=1$. Assim, ao aplicar a ação $a$ em um estado $s$ que satisfaz Precond, existe uma probabilidade $p_{i}$ do efeito $\left(A d d_{i}, D e l_{i}\right) \in$ Effects ocorrer. 


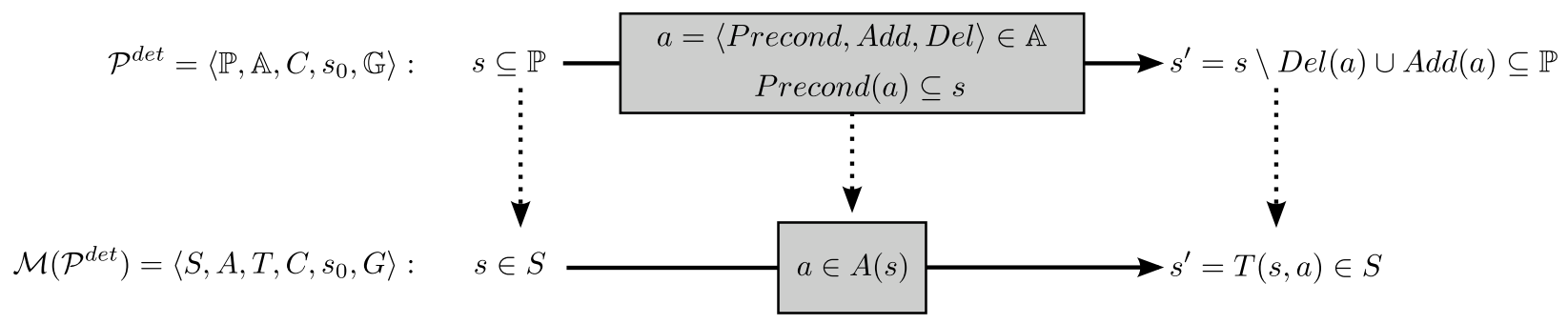

Figura 2.7: Interpretação do problema de planejamento como um modelo de transição de estados.

Definição 10 (Problema de planejamento probabilístico). Um problema de planejamento probabilístico descrito por uma linguagem de ações é definido por uma tupla $\mathcal{P}^{\text {prob }}=\left\langle\mathbb{P}, \mathbb{A}, C, s_{0}, \mathbb{G}\right\rangle$, em que $\mathbb{P}, s_{0}$ e $\mathbb{G}$ são definidos tal como em um problema de planejamento determinístico (Definição 8), e:

- $\mathbb{A}$ é um conjunto de ações probabilísticas, sendo a $\in \mathbb{A}$ especificada pela Equação 2.15.

$\triangle$

Dado um problema de planejamento probabilístico $\mathcal{P}^{\text {prob }}=\left\langle\mathbb{P}, \mathbb{A}, C, s_{0}, \mathbb{G}\right\rangle$ descrito por uma linguagem de ações (Definição 10), podemos inferir o modelo de transição de estados probabilístico (que corresponde a um GMDP da Definição 6) $\mathcal{M}\left(\mathcal{P}^{\text {prob }}\right)=\left\langle S, A, P, C, s_{0}, G\right\rangle$ em que $S, A, C$, so , e $G$ são inferidos como em um problema determinístico (Definição 9) e:

- $P$ é a função de transição probabilística como em um GMDP e $P\left(s_{i} \mid s, a\right)=p_{i}$, em que $s_{i}=$ $\left(s \backslash D e l_{i}\right) \cup A d d_{i}$, para o efeito $p_{i}:\left(A d d_{i}, D e l_{i}\right)$ de $a \in \mathbb{A}$.

\subsubsection{Ações não-determinísticas}

Outro modelo que lida com incerteza nos efeitos das ações é o planejamento não-determinístico, em que não existem probabilidades associadas aos efeitos das ações (no Capítulo 5 discutimos esse problema com mais detalhes). Neste caso, as ações também são dadas por uma lista de efeitos $\left(A d d_{i}, D e l_{i}\right)$, como nas ações probabilísticas, porém, sem especificar as probabilidades $p_{i}$, isto é:

$$
a=\langle\text { Precond, Effects }\rangle \text {. }
$$

Sendo que Effects é uma lista de efeitos na forma Effects $=\left[\left(A d d_{1}\right.\right.$, Del $\left.\left._{1}\right), \ldots,\left(A d d_{n}, D e l_{n}\right)\right]$. Assim, ao aplicar a ação $a$ em um estado $s$ que satisfaz Precond, um dos efeitos $\left(A d d_{i}, D e l_{i}\right) \epsilon$ Effects ocorre, sem nenhuma preferência entre eles.

Definição 11 (Problema de planejamento não-determinístico). Um problema de planejamento não-determinístico totalmente observável (Fully Observable Non-Deterministic Planning Task - FOND) é definido por uma tupla $\mathcal{P}^{\text {nd }}=\left\langle\mathbb{P}, \mathbb{A}, C, s_{0}, \mathbb{G}\right\rangle$, em que $\mathbb{P}, s_{0}$ e $\mathbb{G}$ são definidos tal como em um problema de planejamento deterministico (Definição 8), e:

- $\mathbb{A}$ é um conjunto de ações não-determinísticas, sendo a $\in \mathbb{A}$ especificada pela Equação 2.16. $\triangle$

Dado um problema de planejamento não-determinístico $\mathcal{P}^{n d}=\left\langle\mathbb{P}, \mathbb{A}, C, s_{0}, \mathbb{G}\right\rangle$ descrito por uma linguagem de ações (Definição 11), também podemos inferir o modelo de transição de estados não-determinístico $\mathcal{M}\left(\mathcal{P}^{n d}\right)=\left\langle S, A, T, C, s_{0}, G\right\rangle$ em que $S, A, C, s_{0}$, e $G$ são inferidos como em um problema determinístico (Definição 9 ) e:

- $T: S \times A \rightarrow 2^{S}$ é uma função de transição não-determinística, sendo que $T(s, a)=\left\{s_{i} \mid s_{i}=\right.$ $\left.\left(s \backslash D e l_{i}\right) \cup A d d_{i}, 1 \leq i \leq n\right\}, \forall s \in S, \forall a \in A(s)$; e $n$ é o número de efeitos da ação nãodeterminística $a$.

Assim como em planejamento probabilístico, a solução para um FOND é dada por uma política $\pi: S \rightarrow A$. 
Estados alcançáveis a partir de um estado $s$. Dado um problema de planejamento nãodeterminístico (Definição 11) (ou probabilístico (Definição 10)) e um estado $s \subseteq \mathbb{P}$, o conjunto de estados sucessores de $s$ por uma ação $a \in \mathbb{A}$ é dado por $\operatorname{Suc}(s, a)=\left\{s_{i} \mid s_{i}=\left(s \backslash D e l_{i}\right) \cup A d d_{i}, \forall i \epsilon\right.$ $(1,2, \cdots, n)\}$, sendo $n$ o número de efeitos de $a$. O conjunto dos estados alcançáveis a partir de um estado $s, A l c(s)$, pode ser definido de forma recorrente como:

$$
\operatorname{Alc}(s)=\bigcup_{a \in \mathbb{A}}\left(\operatorname{Suc}(s, a) \cup \bigcup_{s^{\prime} \in \operatorname{Suc}(s, a)} \operatorname{Alc}\left(s^{\prime}\right)\right) .
$$

Podemos definir ainda o conjunto de estados alcançáveis a partir de um estado $s \in S$ por uma política $\pi$ como:

$$
\operatorname{Alc}(s, \pi)=\operatorname{Suc}(s, \pi(s)) \cup \bigcup_{s^{\prime} \in \operatorname{Suc}(s, \pi(s))} \operatorname{Alc}\left(s^{\prime}, \pi\right) .
$$

\subsection{SSP MDPs e becos-sem-saída}

Como vimos no Capítulo 1, quando lidamos com problemas orientados à meta, podemos nos deparar com becos-sem-saída. Na Figura 2.8(a) o estado $d_{2}$ é um exemplo de beco-sem-saída, uma vez que nenhuma política leva o agente de $d_{2}$ para o estado meta $s_{g}$.

Por definição, dado um beco-sem-saída $s$, não existe política $s$-própria, então, um GMDP com pelo menos um beco-sem-saída, não possui política própria e assim não satisfaz a suposição S1 (Definição 7). Isto é, um GMDP com beco-sem-saída não é um SSP MDP.

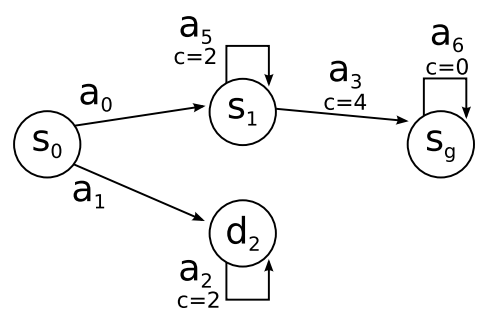

(a)

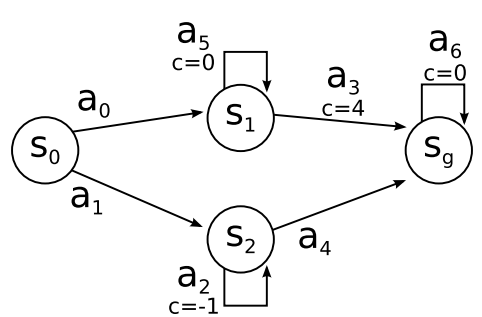

(b)

Figura 2.8: Exemplos de GMDPs que não satisfazem as suposições do SSP MDP.

Uma outra implicação das suposições feitas no SSP MDP é a não existência de ciclos com custo nulo ou negativo, uma vez que isso viola a suposição S2. Por exemplo, no GMDP da Figura 2.8(b), aplicar a ação $a_{5}$ no estado $s_{1}$ cria um ciclo de custo nulo, enquanto aplicar a ação $a_{2}$ no estado $s_{2}$ cria um ciclo cujo custo esperado acumulado é infinito negativo. Assim, esse GMDP não é um SSP MDP, pois não satisfaz a suposição S2, apesar de possuir política própria (i.e., satisfazer a suposição S1).

Os algoritmos apresentados neste capítulo se baseiam nas suposições S1 e S2 de SSP MDPs (Definição 7) ao resolverem problemas com horizonte indefinido sem desconto. Por essa razão, esses algoritmos podem falhar ao tentar resolver um GMDP com becos-sem-saída. Surge assim a necessidade de novos modelos que permitam que o agente de planejamento decida o que fazer nesses casos. Por exemplo, na presença de becos-sem-saída, o agente poderia tentar maximizar a chance de alcançar um estado meta, ignorando o custo das ações. Outra possibilidade seria utilizar um fator de desconto que garanta que o custo esperado seja finito, como no critério de MDPs de horizonte infinito. 


\section{Capítulo 3}

\section{Planejamento probabilístico com becos-sem-saída}

SSP MDPs têm sido amplamente estudados pela comunidade de planejamento, com proposta de soluções eficientes, conforme vimos no Capítulo 2. Essas soluções se baseiam nas suposições S1 e S2 (Definição 7) para garantir que a política encontrada seja ótima. No entanto, essas suposições são muito restritivas e não são satisfeitas por muitos problemas de interesse prático, o que faz com que esses algoritmos falhem ou devolvam uma política sub-ótima. Dessa forma, trabalhos recentes propõem novos modelos e algoritmos que relaxam essas suposições e lidam com classes de problemas mais gerais [Kolobov et al., 2011, 2012b; Teichteil-Königsbuch, 2012; Teichteil-Königsbuch et al., 2011].

A Figura 3.1 ilustra uma situação que não é tratada por um SSP MDP (neste capítulo mantemos o padrão de omitir custos unitários das ações). No problema da Figura 3.1 é impossível alcançar o estado meta $s_{g}$ a partir dos estados $d_{1}, d_{2}$ e $d_{3}$, uma vez que nenhuma ação é capaz de levar o agente para o estado meta $s_{g}$ a partir desses estados e portanto eles são becos-sem-saída. Dessa forma, o problema não possui uma política própria, o que faz com que ele não seja um SSP MDP pois viola a suposição S1 (Definição 7).

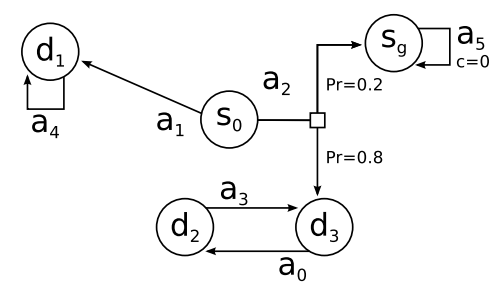

Figura 3.1: Exemplo de GMDP com becos-sem-saída: estados $d_{1}, d_{2}$ e $d_{3}$.

Como dissemos anteriormente, SSP MDPs não modelam problemas que possuem becos-sem-saída. Por outro lado, dado um GMDP, para provar que existe uma política própria basta provar que não existem becos-sem-saída.

SSP MDPs também não modelam problemas com uma função custo qualquer, uma vez que a suposição S2 (Definição 7) diz que toda política imprópria possui um custo esperado infinito em estados com probabilidade menor que um de alcançar um estado meta. Porém, é difícil verificar se essa suposição é satisfeita [Kolobov et al., 2011]. Por exemplo, na Figura 3.1, se $C\left(d_{2}, a_{3}\right)=-1$ (e considerando os demais custos iguais a 1), o valor esperado da política imprópria $\left\{s_{0}: a 2\right\}$ seria 1, pois o laço composto pelos estados $d_{2}$ e $d_{3}$ teria um custo esperado nulo, o que não respeita a suposição S2.

Uma maneira de lidar com problemas que violam as suposições dos SSP MDPs é criar novos modelos e algoritmos que não façam essas suposições restritivas. Neste capítulo, apresentamos classes de GMDPs que relaxam as suposições dos SSP MDPs e podem tratar problemas com becossem-saída. 


\subsection{GMDPs com becos-sem-saída}

Definição 12 (beco-sem-saída). Dado um GMDP $\mathcal{M}=\left\langle S, A, P, C, s_{0}, G\right\rangle$, um estado $s \in S \backslash G$ é um beco-sem-saída se, e somente se, a probabilidade de alcançar um estado meta $s^{\prime} \in G$ a partir de $s$ é zero, isto é, $\forall \pi: P_{\pi}^{G}(s)=0$.

Proposição 2. Um estado $s \in S$ é um beco-sem-saída se o conjunto de estados alcançáveis a partir de $s$ é disjunto do conjunto de estados meta, isto é, $A l c(s) \cap G=\varnothing$.

A Proposição 2 mostra que os becos-sem-saída podem ser definidos de forma independente as probabilidades da função de transição do GMDP. Usaremos essa intuição para caracterizar o conjunto de becos-sem-saída de um GMDP no capítulo 5.

Kolobov et al. [2010] dividem os becos-sem-saída em dois tipos, explícitos e implícitos (como ilustrado no Capítulo 1). Os becos-sem-saída explícitos são estados absorventes (nenhuma ação leva o agente para fora desses estados) e eles não fazem parte do conjunto de estados meta (por exemplo, estado $d_{1}$ da Figura 3.1). Becos-sem-saída implícitos não são estados absorventes, porém não possuem um caminho que os leve para a meta (por exemplo, estados $d_{2}$ e $d_{3}$ da Figura 3.1).

Podemos ainda classificar os problemas de planejamento com becos-sem-saída segundo a sua inevitabilidade, dividindo-os entre evitáveis e inevitáveis. A Figura 3.2 ilustra a diferença entre essas duas classes de problemas. No GMDP da Figura 3.2(a), com beco-sem-saída $d 1$, existe uma política $\pi_{1}=\left\{s_{0}: a_{2}\right\}$ que leva o agente com probabilidade 1 para a meta a partir do estado inicial e dessa forma esse problema possui becos-sem-saída evitáveis. A Figura 3.2(b), mostra um GMDP, com beco-sem-saída $d_{1}$, para o qual nenhuma política leva o agente de $s_{0}$ para $s_{g}$ com probabilidade 1 e nesse caso, o problema possui becos-sem-saída inevitáveis.

Devemos ressaltar que o conceito de evitabilidade não se refere ao estado, mas ao problema. Isto é, um GMDP possui becos-sem-saída evitáveis se existe uma política $s_{0}$-própria, caso contrário, dizemos que o problema possui becos-sem-saída inevitáveis. A Figura 3.2(c) mostra um exemplo de GMDP com dois becos-sem-saída $d_{1}$ e $d_{2}$. Nesse exemplo, é inevitável correr o risco de alcançar um beco-sem-saída a partir de $s_{0}$, no entanto, $d_{1}$ seria evitável pela política $\left\{s_{0}: a_{2}\right\}$ e $d_{2}$ seria evitável pela política $\left\{s_{0}: a_{1}\right\}$. Assim, não definimos que um beco-sem-saída é evitável ou inevitável, mas sim que o GMDP possui becos-sem-saída evitáveis ou inevitáveis.

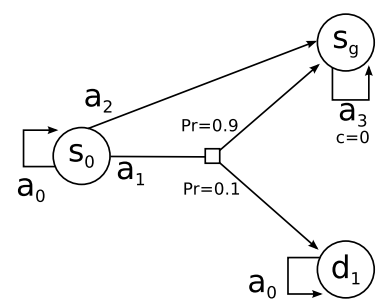

(a)

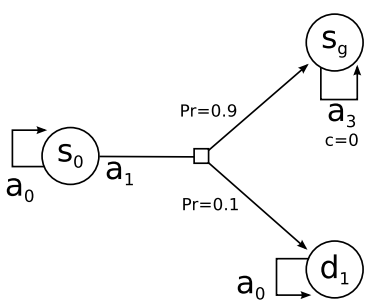

(b)

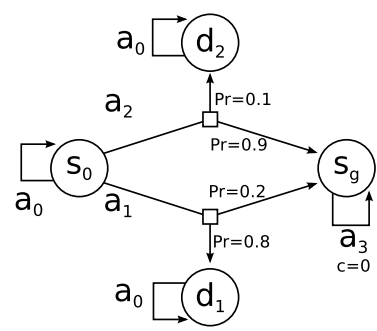

(c)

Figura 3.2: Problemas com becos-sem-saída evitáveis e inevitáveis. (a) GMDP com beco-semsaída evitável; (b) e (c) GMDPs com becos-sem-saída inevitáveis.

Kolobov et al. [2012b] definem duas novas classes de GMDPs que relaxam as suposições dos SSP MDPs permitindo a existência de becos-sem-saída. Além disso, eles definem novos critérios de otimização conforme necessário: o primeiro lida com problemas com becos-sem-saída evitáveis e o segundo lida tanto com becos-sem-saída evitáveis quanto com becos-sem-saída inevitáveis.

\subsubsection{GMDPs com becos-sem-saída evitáveis}

Definição 13 (SSP MDPs com becos-sem-saída evitáveis). Um SSP MDP com Becos-sem-saída Evitáveis (SSP MDP with Avoidable Dead Ends - SSPADE MDP) [Kolobov et al., 2012b] é um GMDP $\mathcal{M}=\left\langle S, A, P, C, s_{0}, G\right\rangle$ que faz as seguintes suposições: 
(A1) $\exists \pi: P_{\pi}^{G}\left(s_{0}\right)=1$, isto é, existe ao menos uma política $s_{0}$-própria; e

(A2) $\forall \pi$ : se $P_{\pi}^{G}\left(s_{0}\right)<1$, então $J_{s_{0}}(\pi)=\infty$, isto é, todas as políticas que não são so-próprias têm custo esperado infinito a partir do estado inicial, sendo $J_{\pi}\left(s_{0}\right)$ dado pela Equação 2.8.

A política ótima $\pi^{*}$ de um SSPADE MDP é uma política $s_{0}$-própria que minimiza o custo esperado dos históricos que alcançam um estado meta partindo do estado inicial $s_{0}$, ou seja:

$$
\pi^{*}=\underset{\pi \text { é } s_{0} \text {-própria }}{\arg \min } C_{\pi}^{G}\left(s_{0}\right) .
$$

Por exemplo, considere três políticas: $\pi_{0}=\left\{s_{0}: a_{0}\right\}, \pi_{1}=\left\{s_{0}: a_{1}, d_{1}: a_{0}\right\}, \pi_{2}=\left\{s_{0}: a_{2}\right\}$ para o GMDP da Figura 3.2(a) (em que todas as ações tem custo igual a 1, exceto a ação $a_{3}$ ). Observamos que $\pi_{2}$ é $s_{0}$-própria $\left(P_{\pi_{2}}^{G}\left(s_{0}\right)=1\right)$, o que satisfaz a suposição A1. Além disso, $\pi_{0}$ e $\pi_{1}$ não são $s_{0}$-próprias $\left(P_{\pi_{0}}^{G}\left(s_{0}\right)=0\right.$ e $\left.P_{\pi_{1}}^{G}\left(s_{0}\right)=0.9\right)$, e como $J_{s_{0}}\left(\pi_{0}\right)=J_{s_{0}}\left(\pi_{1}\right)=\infty$, a suposição A2 também é satisfeita. Logo, esse GMDP é um SSPADE MDP.

Proposição 3. O SSP MDP é uma subclasse do SSPADE MDP.

Demonstração. SSP MDP satisfaz todas as suposições da definição dos SSPADE MDP.

Pela Proposição 3 observamos que todo SSP MDP satisfaz as suposições dos SSPADE MDPs. O diagrama da Figura 3.3 mostra essa relação.

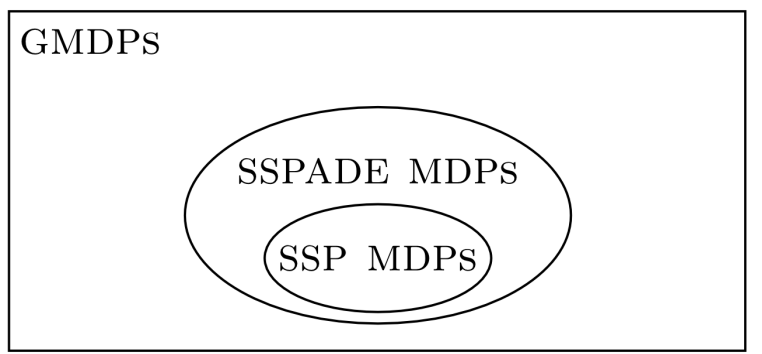

Figura 3.3: Relação entre $\operatorname{SSP} \operatorname{MDP} s$ e SSPADE MDPs.

O algoritmo VI (Seção 2.1.4) não resolve SSPADE MDPs, pois não termina (converge) na presença de becos-sem-saída, uma vez que o valor esperado desses estados $V(s)$ cresce a cada iteração (suposição A2).

O esquema $\mathrm{F} \& \mathrm{R}$ (Seção 2.2) é capaz de resolver SSPADE MDPs. Observe que, à medida que a função valor é atualizada, o custo esperado de becos-sem-saída cresce de forma ilimitada (suposição A2), porém, de acordo com a suposição A1 existe ao menos uma política $s_{0}$-própria, cujo custo esperado a partir de $s_{0}$ é finito. Por exemplo, na Figura 3.2(a) suponha que inicialmente $a_{1}$ é a ação gulosa para $s_{0}$. Como $V\left(d_{1}\right)$ cresce infinitamente, e $V\left(s_{g}\right)=0$ a ação gulosa no estado $s_{0}$ passaria a ser $a_{2}$, evitando assim o beco-sem-saída $d_{1}$.

Apesar de implementar o F\&R, o algoritmo LRTDP (Seção 2.2.1) pode falhar ao resolver um SSPADE MDP uma vez que se um trial alcança um beco-sem-saída, esse trial pode ser infinito. Uma forma simples de contornar esse problema é parar um trial do LRTDP ao visitar um estado pela segunda vez. Note que o ILAO ${ }^{*}$ resolve SSPADE MDPs, pois a expansão do grafo guloso não visita estados repetidos.

\subsubsection{GMDPs com becos-sem-saída inevitáveis}

Outra classe proposta por Kolobov et al. [2012b] trata de GMDPs com becos-sem-saída inevitáveis. Mesmo considerando que toda política que não é $s_{0}$-própria tem um custo esperado infinito a partir do estado inicial, o critério de minimização do custo esperado é inadequado em problemas com becos-sem-saída inevitáveis, pois esse critério não fará distinção entre diferentes políticas 
que não são $s_{0}$-próprias. Por exemplo, dadas duas políticas $\pi_{1}$ e $\pi_{2}, \operatorname{com} P_{\pi_{1}}^{G}\left(s_{0}\right)<P_{\pi_{2}}^{G}\left(s_{0}\right)<1$ e $J_{s_{0}}\left(\pi_{1}\right)=J_{s_{0}}\left(\pi_{2}\right)=\infty$, essas políticas são indistinguíveis pelo critério de minimização do custo esperado a partir de $s_{0}$. No entanto, o agente ainda precisa tomar uma decisão. Observando que $\pi_{2}$ tem maior probabilidade de alcançar um estado meta podemos dizer que essa política é melhor que $\pi_{1}$. Assim, precisamos de um critério de otimização que permita distinguir entre essas duas situações. Kolobov et al. [2012b] propõem dois novos critérios baseados em uma penalidade $D \in \mathbb{R}^{+} \cup\{\infty\}$ atribuída ao agente, caso ele visite um beco-sem-saída. O primeiro trata de problemas em que essa penalidade é finita e o segundo lida com problemas em que ela é infinita.

\subsubsection{Penalidade finita}

No caso de GMDPs com becos-sem-saída inevitáveis e penalidade com valor finito $D$, o agente pode desistir de tentar alcançar um estado meta e pagar esse valor. Assim, o custo esperado é limitado superiormente por $D$. Dada uma penalidade $D \in \mathbb{R}^{+}$atribuída ao agente quando ele não consegue alcançar um estado meta, o valor esperado limitado de uma política $\pi$ a partir de um estado $s$, denotado por $J_{s}^{D}(\pi)$, é dado por:

$$
J_{s}^{D}(\pi)=\min \left[D, J_{s}(\pi)\right] .
$$

Um exemplo prático de problema com penalidade finita por não alcançar a meta é uma usina termoelétrica que precisa alcançar uma meta de produção de energia elétrica produzida por queima de carvão. Para isso, a usina tem a ação produzir, que reduz o estoque de carvão e aumenta a quantidade de energia produzida. Supondo um estoque limitado de carvão, essa usina só poderá produzir enquanto houver combustível. A ação de produzir pode levar o agente para três estados possíveis: meta-alcançada; sem-combustível e com-combustível. Caso a usina não alcance sua meta ela será penalizada com uma multa.

\subsubsection{Penalidade infinita}

No caso de GMDPs com becos-sem-saída inevitáveis e penalidade infinita $D=\infty$, temos que $J_{s}^{D}(\pi)=J_{s}(\pi)$, e voltamos ao problema original de GMDPs com becos-sem-saída inevitáveis em que não podemos distinguir políticas que não são $s_{0}$-próprias. Há duas possibilidades: podemos desconsiderar o custo das ações, nesse caso, utilizamos a probabilidade de alcançar um estado meta a partir de s seguindo uma politica $\pi, P_{\pi}^{G}\left(s_{0}\right)$ (Equação 2.10 ), como uma função objetivo a ser maximizada; ou podemos ignorar o custo de históricos que não alcançam um estado meta, nesse caso, utilizamos o custo esperado dos históricos que alcançam um estado meta a partir de s seguindo uma política $\pi, C_{\pi}^{G}(s)$ (Equação 2.11), como uma função objetivo a ser minimizada. Isto é, em GMDPs que não possuem uma política $s_{0}$-própria, há dois critérios de otimização possíveis: $(i)$ maximização de $P_{\pi}^{G}\left(s_{0}\right)$ (Equação 2.10) e (ii) minimização de $C_{\pi}^{G}\left(s_{0}\right)$ (Equação 2.11).

Esse problema poderia ser visto como um problema de decisão sequencial multiobjetivo, cuja solução é o conjunto de políticas do pareto front ${ }^{1}$ [Roijers et al., 2013]. A Figura 3.4 mostra a probabilidade de alcançar um estado meta a partir do estado $s_{0}$ e o custo esperado dos caminhos que terminam em um estado meta das políticas do pareto front de um dado GMDP. Por exemplo, a política $\pi_{1}$ tem probabilidade de alcançar a meta a partir do estado inicial de 0.4 com custo dos esperado dos históricos que alcançam a meta de 1.46. Note que, nesse exemplo, $\pi_{1}$ domina todas as políticas com $C_{\pi}^{G}\left(s_{0}\right)=1.46$, isto é, entre as políticas com $C_{\pi}^{G}\left(s_{0}\right)=1.46, \pi_{1}$ é a que tem maior probabilidade. De forma semelhante, $\pi_{1}$ domina todas as políticas com $P_{\pi}^{G}\left(s_{0}\right)=0.4$, isto é, entre as políticas com $P_{\pi}^{G}\left(s_{0}\right)=0.4, \pi_{1}$ é a que tem menor custo esperado dos históricos que alcançam a meta a partir de $s_{0}$. Note que um critério mais razoável é maximizar a probabilidade de alcançar a meta antes de minimizar o custo dos históricos que levam para a meta, uma vez que probabilidades

\footnotetext{
${ }^{1}$ Pareto front é o conjunto de políticas que não são dominadas por nenhuma outra política. Uma política $\pi$ domina uma política $\pi^{\prime}$ se para todos os objetivos, $\pi$ é ao menos tão boa quanto $\pi^{\prime}$ e estritamente melhor que $\pi^{\prime}$ em pelo menos um objetivo.
} 


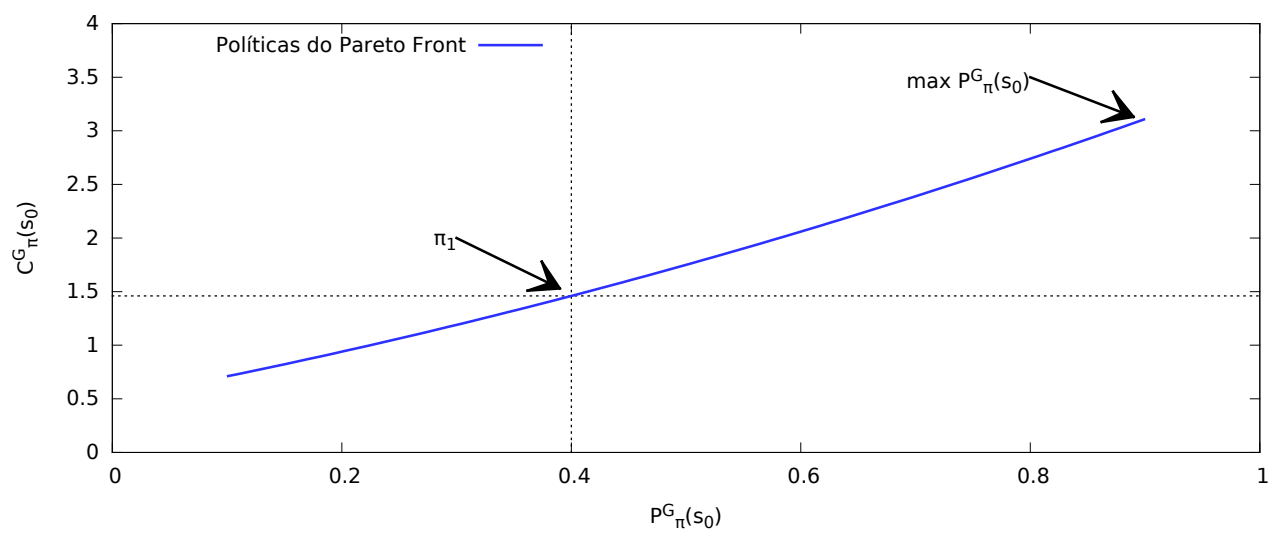

Figura 3.4: Utilidade das melhores políticas de um problema multiobjetivo. O gráfico mostra a probabilidade de alcançar um estado meta e o custo dos históricos que alcançam um estado meta das politicas do pareto front. A seta da direita indica a melhor política quando consideramos que a probabilidade de alcançar um estado meta tem uma preferência maior.

menores aumentam o risco do agente receber uma penalidade infinita. Por isso, maximizar $P_{\pi}^{G}(s)$ tem preferência sobre minimizar $C_{\pi}^{G}(s)$. Isso permite impor uma ordem entre os objetivos (definida a seguir). Assim, na Figura 3.4 a política ótima seria a política do pareto front que maximiza a probabilidade de alcançar um estado meta, isto é, $\pi^{*}=\underset{\pi \in \text { pareto front }}{\arg \max } P_{\pi}^{G}(s)$.

Kolobov et al. [2012b] propõem o uso de um critério de otimização ordenado por preferência através de uma ordem lexográfica sobre os objetivos. Esse critério primeiro maximiza a probabilidade de alcançar a meta, $P_{\pi}^{G}(s)$ (Equação 2.10), e em seguida minimiza o custo esperado entre históricos que alcançam a meta, $C_{\pi}^{G}(s)$ (Equação 2.11). Assim, dado um GMDP com penalidade infinita, o valor esperado de uma política $\pi$ a partir de um estado $s$, denotado por $J_{s}^{\infty}(\pi)$, é dado por um par ordenado:

$$
J_{s}^{\infty}(\pi)=\left(P_{\pi}^{G}(s), C_{\pi}^{G}(s)\right),
$$

que define dois critérios de avaliação de uma política com base numa relação de ordem < entre duas políticas $\pi_{1}$ e $\pi_{2}$, sendo que, $\pi_{1}(s)<\pi_{2}(s)$ denota que $\pi_{2}$ é preferível em relação a $\pi_{1}$ em $s$ : $\pi_{1}(s)<\pi_{2}(s)$ quando $P_{\pi_{2}}^{G}(s)>P_{\pi_{1}}^{G}(s)$ ou, quando $P_{\pi_{2}}^{G}(s)=P_{\pi_{1}}^{G}(s)$ e $C_{\pi_{2}}^{G}(s)<C_{\pi_{1}}^{G}(s)$. Assim, a política ótima $\pi^{*}$ é a de maior preferência segundo $<$, ou seja:

$$
\pi^{*}=\underset{<\pi}{\arg \max } J_{s_{0}}^{\infty}(\pi)=\underset{<\pi}{\arg \max }\left(P_{\pi}^{G}(s), C_{\pi}^{G}(s)\right),
$$

onde $\max _{<\pi}$ é o operador de maximização segundo a relação de ordem <. O cálculo de $\pi^{*}$ também pode ser definido em dois passos consecutivos:

$$
\begin{aligned}
& \text { Passo 1: } \Pi_{\text {maxprob }}=\left\{\pi^{\prime} \mid \pi^{\prime}=\underset{\pi}{\arg \max } P_{\pi}^{G}(s)\right\}, \\
& \text { Passo 2: } \pi^{*}=\underset{\pi \in \Pi_{\text {maxprob }}}{\arg \min } C_{\pi}^{G}(s) .
\end{aligned}
$$

Um exemplo de problema com penalidade infinita por não alcançar a meta é o problema do limpador de janelas de um arranha-céus. O limpador pode decidir entre trabalhar com diferentes equipamentos de segurança envolvendo diferentes custos e, em caso de queda, existe alta probabilidade de morte. Naturalmente, não é possível definir um valor finito para $D$ nesse problema. Assim, espera-se que a pessoa maximize sua probabilidade de alcançar a meta optando por utilizar um dos equipamentos de segurança mais confiáveis (com menor probabilidade de falha) e, entre esses equipamentos, o equipamento de menor custo. 
Definição 14 (SSP MDP com Becos-sem-saída Inevitáveis (SSP MDP with Unavoidable Dead Ends - SSPUDE MDP)). Um SSPUDE MDP [Kolobov et al., 2012b] é uma tupla $\mathcal{M}=$ $\left\langle S, A, P, C, s_{0}, G, D\right\rangle$ em que $S, A, P, C, s_{0}$ e $G$ são definidos como em um GMDP (Definição 6) e:

- $D \in \mathbb{R}^{+} \cup\{\infty\}$ é a penalidade atribuída ao agente quando ele visita um beco-sem-saída.

Se $D<\infty$, então $\mathcal{M}$ é um SSPUde MDP com penalidade finita ( $f$ SSPUDE MDP). A solução ótima de um fSSPUDE MDP é uma política que minimiza o custo esperado limitado a partir do estado inicial $s_{0}$, isto é $\pi^{*}=\arg \min J_{s_{0}}^{D}(\pi)$ (Equação 3.2).

Se $D=\infty$, então $\mathcal{M}$ é um SSPUDE MDP com penalidade infinita (iSSPUDE MDP). A solução ótima de um iSSPUDE MDP é uma política $\pi^{*}=\underset{\pi \in \Pi_{\text {maxprob }}}{\arg \min } C_{\pi}^{G}(s)$, conforme as equações 3.5 e 3.6.

A Figura 3.5 mostra exemplos de SSPUDE MDPs. Cada um desses problemas possui três políticas: $\pi_{0}=\left\{s_{0}: a_{0}\right\}, \pi_{1}=\left\{s_{0}: a_{1}, d_{1}: a_{0}\right\}$ e $\pi_{2}=\left\{s_{0}: a_{2}, d_{2}: a_{0}\right\}$ (exceto o primeiro que só tem as políticas $\pi_{0}$ e $\left.\pi_{1}\right)$. Nota-se que em nenhum destes problemas existe uma política $s_{0}$-própria, portanto, esperase que o agente opte por aplicar uma ação que possa levá-lo para a meta, assumindo o menor risco de cair nos becos-sem-saída $d_{1}$ e $d_{2}$. Analisando cada problema pelo critério do iSSPUDE MDP (Equação 3.3) percebemos que:

- Na Figura 3.5(a) o agente deve optar pela ação $a_{1}$ em $s_{0}$ com uma chance de $90 \%$ de alcançar o estado meta $s_{g}$, ao invés da ação $a_{0}$ que nunca leva o agente para a meta.

- Na Figura 3.5(b) há duas políticas que podem levar o agente para o estado meta $s_{g}$, e ele deve optar pela ação $a_{2}$ em $s_{0}$, que o leva para a meta $90 \%$ das vezes, ao invés da ação $a_{1}$, que só o levaria para a meta $20 \%$ das vezes.

- Na Figura 3.5(c), as duas políticas $\pi_{1}$ e $\pi_{2}$ têm a mesma probabilidade de alcançar o estado meta $s_{g}$, e o agente deve optar pela política $\pi_{2}$ que tem um menor custo esperado dos históricos que alcançam a meta.

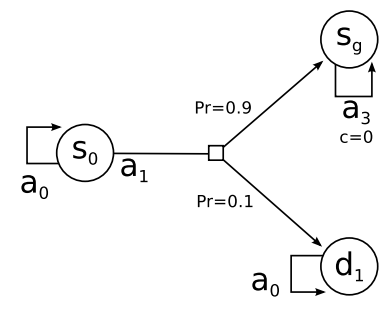

(a)

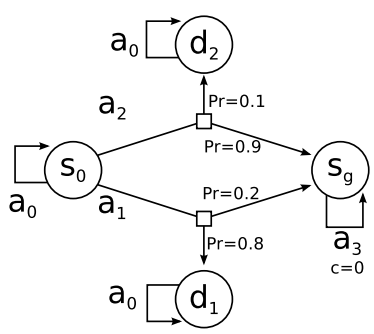

(b)

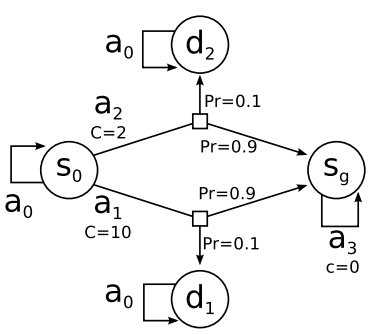

(c)

Figura 3.5: Exemplos de SSPUDE MDP s em que: (a) apenas uma ação leva ao estado meta; (b) ações distintas podem levar ao estado meta com probabilidade diferentes. (c) ações distintas levam ao estado meta com a mesma probabilidade, mas com custos diferentes.

Proposição 4. iSSPUDE MDP é uma generalização do SSPADE MDP (Definição 13)[Kolobov et al., 2012b].

Demonstração. Queremos demonstrar que ao aplicarmos o critério de otimização dos iSSPUDE MDPs (Equação 3.3) em um SSPADE MDP, este devolve a política ótima do SSPADE MDP (Equação 3.1). Dado um iSSPUDE MDP $\mathcal{M}$, se $\mathcal{M}$ possuir políticas $s_{0}$-próprias, estas serão as políticas de maior preferência, uma vez que $P_{\pi}^{G}\left(s_{0}\right)=1$. Em seguida, $\min _{\pi} C_{\pi}^{G}\left(s_{0}\right)$ é usado como critério de desempate entre as políticas $s_{0}$-próprias. Assim, ao resolvermos um SSPADE MDP $\mathcal{M}=\left\langle S, A, P, C, s_{0}, G\right\rangle$ como um $i$ SSPude $\operatorname{mdp} \mathcal{M}^{\prime}=\left\langle S, A, P, C, s_{0}, G, \infty\right\rangle$ obtemos uma política ótima para $\mathcal{M}$. 


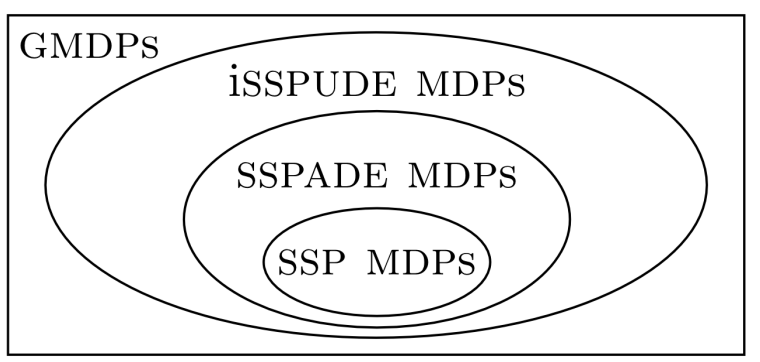

Figura 3.6: Relação entre $\operatorname{SSPADE} \operatorname{MDP} s$ e $i \operatorname{SSPUDE} \operatorname{MDP} s$.

Pela Proposição 4 observamos que o critério de otimização dos iSSPUDE MDPs é capaz de encontrar a política ótima para SSPADE MDPs. O diagrama da Figura 3.6 mostra essa relação, incluindo também a relação entre SSPADE MDPs e SSP MDPs conforme a Figura 3.3.

Proposição 5. A classe de fSSPUDE MDPs é igual à classe de SSP MDPs [Kolobov et al., 2012b].

Demonstração. Ida: Dado um $f$ SSPUde MDPs $\mathcal{M}=\left\langle S, A, P, C, s_{0}, G, D\right\rangle$ podemos criar um GMDP $\mathcal{M}^{\prime}=\left\langle S, A^{\prime}, P, C, s_{0}, G\right\rangle$, em que $A^{\prime}=A \cup a^{\prime}$, sendo $a^{\prime}$ uma nova ação, com custo $D$ e aplicável em todo estado $s \notin G$, que leva para um estado de $G$. A política ótima de $\mathcal{M}^{\prime}$ segundo o critério de otimização do SSP MDP é igual a política ótima de $\mathcal{M}$.

Volta: Dado um SSP mDP $\mathcal{M}=\left\langle S, A, P, C, s_{0}, G\right\rangle$, criamos um $f$ Sspude mdp $\mathcal{M}=\left\langle S, A, P, C, s_{0}, G, D\right\rangle$, sendo $D=\max _{s \in S} V^{*}(s)$. A política ótima de $\mathcal{M}^{\prime}$ é igual a política ótima de $\mathcal{M}$.

Teichteil-Königsbuch [2012] propôs o problema de caminho estocástico mínimo mais seguro (Stochastic Safest Shortest Path $-\mathrm{S}^{3} \mathrm{P} \mathrm{MDP}$ ), definindo um modelo para planejamento probabilístico com becos-sem-saída cujo critério de otimização, segundo Kolobov [2013, p. 215], é análogo ao do iSSPUDE MDP.

\subsubsection{Algoritmos para SSPUDE MDPs}

Para resolver $f$ SSPUDE MDP, Kolobov et al. [2012b] modificam o algoritmo VI com uma atualização que limita o valor esperado, isto é:

$$
V^{t}(s) \leftarrow \min \left[D, \min _{a \in A(s)}\left\{C(s, a)+\sum_{s^{\prime} \in S} P\left(s^{\prime} \mid s, a\right) V^{t-1}\left(s^{\prime}\right)\right\}\right],
$$

com $V^{0}(s)=0$ se $s \in G$. É possível mostrar que a Equação 3.7 converge para $V^{*}(s)=J_{s}^{D}\left(\pi^{*}\right)$ onde $\pi^{*}$ é a política ótima do $f$ SSPUDE MDP (Definição 14), considerando uma função custo positiva ${ }^{2}$.

Para resolver iSSPUDE MDPs, Kolobov et al. [2012b] e Teichteil-Königsbuch [2012] propuseram um método que computa a política ótima $\pi^{*}$ segundo as Equações 3.5 e 3.6: o primeiro passo implementa o algoritmo VI desconsiderando o custo das ações e o segundo passo também implementa o algoritmo VI, mas só considera as políticas de $\Pi_{\text {maxprob }}$. O Apêndice A descreve esse algoritmo com mais detalhes.

Uma característica interessante desses modelos é que, caso a função custo seja positiva, a política ótima de um iSSPUDE MDP $\mathcal{M}=\left\langle S, A, P, C, s_{0}, G, \infty\right\rangle$ é equivalente à política ótima de um $f$ SSPUDE MDP correspondente, isto é, um $f$ SSPUDE MDP $\mathcal{M}^{\prime}=\left\langle S, A, P, C, s_{0}, G, D\right\rangle$, com um valor de penalidade $D$ suficientemente grande. Kolobov et al. [2012b] provaram que, dado um $i$ SSPUDE MDP $\mathcal{M}$, existe um valor $D_{\text {limite }}$ a partir do qual, a política ótima do $f$ SSPUDE MDP correspondente com $D>D_{\text {limite }}$ é a política ótima do $i$ SSPUDE MDP $\mathcal{M}$. Nos experimentos realizados no Capítulo 7 , usaremos essa ideia para resolver $i$ SSPUDE MDPs

\footnotetext{
${ }^{2}$ Apesar de Kolobov et al. [2012b] não fazerem essa suposição, é fácil perceber que o algoritmo não converge caso haja um ciclo com custo negativo
} 


\subsection{Outras classes de GMDPs}

$\mathrm{Na}$ seção anterior foram apresentadas extensões do SSP MDP que permitem a existência de becos-sem-saída. No entanto, existem outros modelos que também podem tratar esse tipo de problema. A seguir, analisamos alguns deles e as principais diferenças para os modelos apresentados anteriormente.

\subsubsection{GMDP com desconto}

Conforme vimos na Seção 2.1.1, num MDP de horizonte infinito, uma forma de evitar que o valor de um estado seja infinito, é utilizar um custo esperado descontado a partir do estado inicial $J_{s_{0}}(\pi)$ (Equação 2.8). Com isso, Teichteil-Königsbuch et al. [2011] definiram uma nova classe de GMDPs que utiliza esse critério para lidar com becos-sem-saída e não faz nenhuma suposição. Chamamos esse modelo de GMDP com desconto.

Definição 15 (GMDP com desconto). Um GMDP com desconto é uma tupla $\mathcal{M}=\left\langle S, A, P, C, s_{0}, G, \gamma\right\rangle$ em que $S, A, T, C, s_{0}$ e $G$ são definidos como em um GMDP (Definição 6) e:

- $0<\gamma<1$ é o fator de desconto que pondera entre custo imediato e futuro.

A política ótima de um GMDP com desconto minimiza o custo esperado descontado a partir do estado inicial $s_{0}$ :

$$
\pi^{*}=\underset{\pi}{\arg \min } J_{s_{0}}(\pi) .
$$

Para resolver um GMDP com desconto basta utilizar algoritmos de programação dinâmica que resolvem MDPs de horizonte infinito, por exemplo o algoritmo VI (Equação 2.9) (Note que para resolver SSP MDPs não descontamos os custos futuros, $\gamma=1$, enquanto para resolver GMDPs com desconto os custos futuros são sempre descontados, $\gamma<1$ ).

Note que algoritmos de programação dinâmica descontada também podem encontrar a solução ótima de GMDPs em geral: SSP, SSPADE e SSPUDE MDPs, dado um valor de $\gamma$ adequado (considerando a função custo positiva). No entanto, encontrar o $\gamma$ adequado é um problema complexo. No Apêndice B demonstramos, para um dado SSP MDP, como a distância para a meta pode tornar políticas próprias e impróprias indistinguíveis, usando o critério de custo esperado descontado dos GMDPs com desconto.

\subsubsection{SSP MDP generalizado - GSSP}

O SSPADE MDP, bem como sua subclasse SSP MDP, assumem que $J_{s_{0}}(\pi)=\infty$ para toda política $\pi$ que não é $s_{0}$-própria. Porém, essa suposição ainda pode ser relaxada. Kolobov et al. [2011] definiram o SSP MDP Generalizado (Generalized SSP MDP - GSSP MDP) que estende o SSPADE MDP para problemas que possuem políticas $\pi$ que não são $s_{0}$-próprias e $J_{s_{0}}(\pi) \neq \infty$.

Seja $h=\left\langle s_{0}, s_{1}, \cdots\right\rangle$ um histórico infinito obtido seguindo uma política $\pi$. O custo negativo acumulado desse histórico, denotado por $C_{\pi}^{-}(h)$, é dado por:

$$
C_{\pi}^{-}(h)=\sum_{i \geq 0} \min \left\{0, C\left(s_{i}, \pi\left(s_{i}\right)\right)\right\}
$$

Seja $\mathcal{H}_{s}$ o conjunto de todos os históricos cujo primeiro estado é $s \in S$. O custo negativo esperado do estado $s$ seguindo uma política $\pi$ é a soma dos custos negativos acumulados dos históricos de $\mathcal{H}_{s}$ ponderados pela probabilidade de cada histórico, isto é:

$$
C_{\pi}^{-}(s)=\sum_{h \in \mathcal{H}_{S}} C_{\pi}^{-}(h) P(h \mid \pi) .
$$

Definição 16 (SSP MDP Generalizado (Generalized SSP MDP - GSSP MDP)). Um GSSP MDP é um GMDP (Definição 6) $\mathcal{M}=\left\langle S, A, P, C, s_{0}, G\right\rangle$ que faz as seguintes suposições: 
(G1) $\exists \pi: P_{\pi}^{G}\left(s_{0}\right)=1$, isto é, existe pelo menos uma política $s_{0}$-própria; e

(G2) $\forall \pi: C_{\pi}^{-}\left(s_{0}\right)>-\infty$, isto é, a soma dos custos negativos gerados por uma política a partir do estado inicial $s_{0}$ é maior que $-\infty$.

Intuitivamente, a suposição G2 diz que o custo negativo acumulado por uma política $\pi$ a partir de $s_{0}$ é sempre finito. Para esse modelo o critério de otimização das políticas é descrito apenas sobre as políticas próprias a partir de $s_{0}$ e busca minimizar o valor esperado a partir do estado inicial:

$$
\pi^{*}=\underset{\pi \text { é } s_{0} \text {-própria }}{\arg \min } J_{s_{0}}(\pi) .
$$

A Figura 3.7 mostra dois exemplos de GMDPs para os quais existem três políticas possíveis: $\pi_{1}=\left\{s_{0}: a_{0}, s_{1}: a_{3}\right\}, \pi_{2}=\left\{s_{0}: a_{0}, s_{1}: a_{2}\right\}$ e $\pi_{3}=\left\{s_{0}: a_{1}, s_{2}: a_{2}\right\}$. Nos dois exemplos $P_{\pi_{1}}^{G}\left(s_{0}\right)=1$, $\pi_{1}$ é um política $s_{0}$-própria ela satisfaz G1. As demais políticas não são $s_{0}$-próprias. Entretanto, como $C_{s_{0}}^{-}\left(\pi_{2}\right)=C_{s_{0}}^{-}\left(\pi_{3}\right)>-\infty$, esses GMDPs satisfazem G2. Logo, ambos são GSSP MDPs. Notamos assim que GSSP MDPs permitem a presença de ciclos com custo nulo (por exemplo, os estado $s_{1}$ e $s_{2}$ aplicando a ação $a_{2}$ da Figura 3.7(a)), ou custo esperado finito a partir do estado inicial: $J_{\pi_{2}}\left(s_{0}\right)=1$ (Figura 3.7(a)) e $J_{\pi_{2}}\left(s_{0}\right)=-6$ (Figura $3.7(\mathrm{~b})$ ).

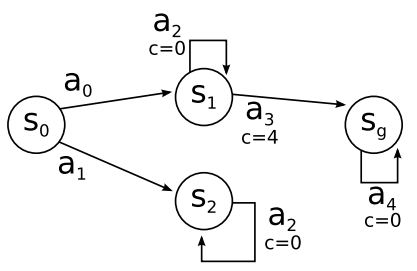

(a)

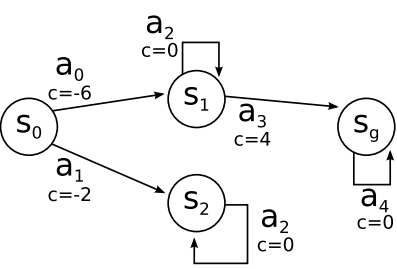

(b)

Figura 3.7: Exemplos de GSSP MDPs.

A Figura 3.8 mostra dois exemplos de GMDPs que não são GSSP MDPs. Os dois exemplos possuem duas políticas: $\pi_{1}=\left\{s_{0}: a_{0}, s_{1}: a_{1}\right\}$ e $\pi_{2}=\left\{s_{0}: a_{0}, s_{1}: a_{2}\right\}$. Apesar de ambos os problemas satisfazerem a suposição $\mathrm{G} 1$, pois possuem política própria $\left(\pi_{1}\right)$, eles não satisfazem a suposição G2, pois $C_{s_{0}}^{-}\left(\pi_{2}\right)=-\infty$.

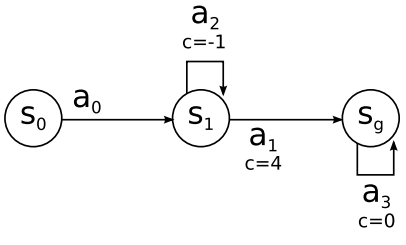

(a)

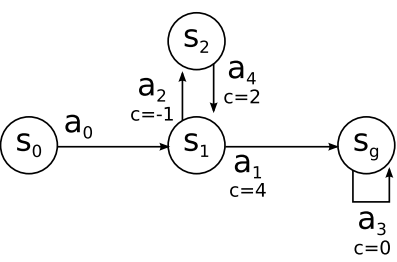

(b)

Figura 3.8: Exemplos de GMDPs que não satisfazem as suposições dos GSSP MDPs.

Ciclos com custo nulo, por exemplo os estado $s_{1}$ e $s_{2}$ aplicando a ação $a_{2}$ da Figura 3.7(a), têm o mesmo comportamento de um estado meta, esse tipo de ciclo é chamado trap. Algoritmos de programação dinâmica, como o LRTDP, ao serem aplicados em GMDPs com esse tipo de ciclo, podem obter políticas que optam por ir para esses estados ao invés de ir para um estado meta. Para que esses algoritmos devolvam uma política $s_{0}$-própria (que sempre alcança um estado meta), é necessário adaptá-los para identificar esses estados e evitá-los.

Uma trap é uma SCC $F=\left\{S_{F}, A_{F}\right\}$ de um grafo guloso $G_{\pi}$ com as seguintes propriedades: $S_{F} \subseteq A l c\left(s_{0}, \pi\right) ; S_{F} \cap G=\varnothing ;$ e $\forall\left(s_{i}, s_{j}\right) \in A_{F} \rightarrow s_{i}$ e $s_{j} \in S_{F}$. Isto é, uma trap é uma SCC $F$ de um grafo guloso de uma política $\pi$ a partir do estado inicial $s_{0}$ que não possui nenhum estado meta, no qual todos os estados são alcançáveis a partir do estado inicial e todas as arestas levam para algum estado dentro da componente. Há dois tipos de traps: 
Traps transientes: se considerarmos ações que não fazem parte da trap (ações que não pertencem ao grafo guloso $G_{\pi}$ ) é possível alcançar estados fora da trap; e

Traps permanentes: mesmo considerando ações que não fazem parte da trap, não é possível alcançar estados que não pertencem a essa trap. Assim, mesmo considerando ações que não fazem parte de $\pi$ não é possível alcançar um estado meta.

O exemplo da Figura 3.7(a) mostra um GMDP que pode ter duas traps, dependendo da política escolhida: a primeira $F_{1}=\left\{\left\{s_{1}\right\},\left\{\left(s_{1}, s_{1}\right)\right\}\right\}$ e a segunda $F_{2}=\left\{\left\{s_{2}\right\},\left\{\left(s_{2}, s_{2}\right)\right\}\right\}$. Nesse exemplo, $F_{1}$ é uma trap transiente pois no estado $s_{1}$ é possível aplicar a ação $a_{3}$ que leva para $s_{g}$ que não pertence a $F_{1}$. Por outro lado, $F_{2}$ é uma trap permanente, pois não há outras ações que levem para estados fora dessa trap.

Relacionando traps e becos-sem-saída, observamos que traps permanentes são compostas exclusivamente de becos-sem-saída e que nem todo estado de uma trap transiente é um beco-sem-saída.

Para resolver GSSP MDPs, [Kolobov et al., 2011] propôs o esquema FRET (Find, Revise and Eliminate Traps). Esse esquema é dividido em duas etapas:

1. Encontrar e revisar: nesta etapa é computada uma função valor ótima $V^{*}$ seguindo o esquema F\&R (Seção 2.2), utilizando um algoritmo como o LRTDP;

2. Eliminar traps: nesta etapa são detectadas as traps permanentes e transientes. Para eliminálas, altera-se a função valor dos estados das traps permanentes para infinito, e o valor dos estados das traps transientes é incrementado com o menor custo de sair dessa trap, considerando as ações que não fazem parte da política $\pi$.

Essas duas etapas são repetidas até que nenhuma trap seja encontrada no grafo guloso.

\section{Relação entre GSSPs e outros modelos.}

- A suposição G1 dos GSSP MDPs diz que GSSP MDPs tratam somente problemas com becossem-saída evitáveis, logo os GSSP MDPs não lidam com SSPUDE MDPs.

- Os SSPUDE MDPs também tratam problemas com becos-sem-saída evitáveis, incluindo tanto problemas com traps permanentes quanto problemas com traps transientes, pois esse modelo primeiramente maximiza a probabilidade de alcançar um estado meta, ignorando ações que possam gerar uma trap.

- SSPADE MDPs não tratam problemas com ciclos de custo nulo, logo, incluem apenas GMDPs com traps permanentes nas quais todos os estados possuem um custo esperado infinito.

- Nem todo SSP MDP satisfaz as suposições do GSSP MDP, a Figura 3.8(b) mostra um GMDP que satisfaz as suposições do SSP MDP, mas não satisfaz a suposição G2, pois $C_{s_{0}}^{-}\left(\pi_{2}\right)=-\infty$.

- Note que em um SSP MDP, cuja função custo é positiva $\left(C \rightarrow \mathbb{R}^{+}\right)$, o custo acumulado negativo (Equação 3.8) de todos os históricos é sempre 0, o que garante que a suposição G2 seja satisfeita. Além disso, como um SSP MDP tem uma política própria (suposição S1), ele também tem uma política $s_{0}$-própria (suposição G1). Portanto, todo SSP MDP com uma função custo positiva é também um GSSP MDP [Kolobov, 2013].

\subsubsection{Maximização de probabilidade (MAXPROB)}

Outra forma de lidar com GMDPs é considerar apenas a probabilidade de alcançar um estado meta. O MDP de Probabilidade Máxima (Maximum Probability MDP - MAXPROB MDP) procura por uma política que maximiza a probabilidade de alcançar um estado meta (Equação 2.10) a partir do estado inicial $s_{0}$ :

$$
\pi^{*}=\arg \max _{\pi} P_{\pi}^{G}\left(s_{0}\right)
$$


Assim, esse critério pode lidar com problemas que possuem becos-sem-saída.

Recentemente, o MAXPROB MDP tem recebido muita atenção [Camacho et al., 2016; Kolobov et al., 2011; Steinmetz et al., 2016]. Uma forma de resolver esse problema é adaptar algoritmos de programação dinâmica para computar $\max _{\pi} P_{\pi}^{G}\left(s_{0}\right)$. Kolobov et al. [2011] mostraram que é possível reduzir um MAXPROB MDP $\mathcal{M}$ para um GSSP MDP $\mathcal{M}^{\prime}$, sendo que a política ótima de $\mathcal{M}^{\prime}$ é igual a política ótima de $\mathcal{M}$. Steinmetz et al. [2016] propuseram adaptações eficientes para algoritmos de programação dinâmica assíncronos como LRTDP e ILAO* (que seguem o esquema FRET) para resolver esse problema computando a função $\max _{\pi} P^{G}$ e em seguida extraindo a política ótima.

Apesar dessa classe de problemas possuir uma interseção com os SSP MDPs, ela só resolve SSP MDPs em que todas as políticas próprias têm o mesmo custo esperado. Como o critério do MAXPROB MDP não distingue duas políticas que possuem a mesma probabilidade de alcançar um estado meta, esse modelo não resolve todos os SSP MDPs.

Encontrar todas as políticas que maximizam a probabilidade de alcançar um estado meta é um subproblema de outros modelos, tanto dos que assumem a existência de uma política própria, como o SSP MDP, o SSPADE MDP e o GSSP MDP, quanto dos que não assumem, como o iSSPUDE MDP. Assim, uma possibilidade para resolver esses problemas seria identificar todas as políticas que maximizam a probabilidade de alcançar um estado meta e restringir a solução desses problemas para esse subconjunto de políticas. Como mostramos anteriormente, os critérios de otimização do $f$ SSPUDE MDP e do GMDP com desconto podem optar por uma política que não maximiza a probabilidade de alcançar um estado meta, logo, o MAXPROB MDP não é um subproblema desses modelos.

\subsection{Discussão}

Neste capítulo observamos que os modelos para planejamento probabilístico orientados à meta que lidam com becos-sem-saída podem ser divididos em três categorias: $(i)$ modelos que utilizam dois critérios de otimização ordenados; (ii) modelos que ignoram o custo das ações; e (iii) modelos que garantem que o custo esperado é limitado utilizando um hiper-parâmetro $(D$ e $\gamma)$.

A primeira categoria utiliza dois critérios de avaliação de política ordenados: maximizar a probabilidade de alcançar um estado meta e minimizar o custo esperado dos históricos que alcançam a meta, representada pelo modelo iSSPUDE MDP. Esses critérios representam a postura de um agente que coloca em primeiro plano o objetivo de alcançar um estado meta, colocando em segundo plano o custo esperado. Os algoritmos propostos para esses modelos envolvem a aproximação de duas funções separadas, o que pode ser um processo computacionalmente custoso [Kolobov et al., 2012b].

A segunda categoria, representada pelo MAXPROB MDP, também se preocupa em alcançar um estado meta. No entanto, esse modelo ignora os custos. Em geral, esse modelo se aplica quando alcançar a meta é a prioridade, independentemente do custo.

A terceira categoria lida com critérios de otimização baseados em hiper-parâmetros predeterminados $(D$ e $\gamma$ ), representado pelos modelos $f$ SSPUDE MDP e GMDP com desconto. Nesses modelos, a política ótima é definida por uma única função valor, logo só é necessário computar uma função valor ótima. No entanto, conforme vimos nesse capítulo, esses parâmetros não garantem que a política ótima seja aquela que maximiza a probabilidade de alcançar um estado meta. Isto é, se os parâmetros não forem bem definidos, a política devolvida não tem garantia de otimalidade. Por exemplo, se $D=0$, é possível que um beco-sem-saída passe a ser mais atrativo para o agente do que um estado meta, nesse caso a política que minimiza $J_{\pi}^{D}\left(s_{0}\right)$ não seria a que tem maior probabilidade de alcançar um estado meta. No caso dos GMDPs com desconto, se $\gamma$ for muito próximo de zero, o agente não saberá distinguir entre políticas próprias e impróprias, nesse caso, assim como no exemplo anterior, a política que minimiza $J_{\pi}\left(s_{0}\right)$ pode não ser a de maior probabilidade de alcançar um estado meta.

Kolobov et al. [2012b] demonstraram que é possível resolver um $i$ SSPUDE MDP como um $f$ SSPUDE MDP com uma penalidade adequada $\left(D \geq D_{\text {thres }}\right)$. De um lado, a eficiência dessa abordagem de- 
pende do valor de $D$, isto é, se utilizarmos um valor muito alto o algoritmo pode tomar muito tempo, fazendo muitas atualizações em um beco-sem-saída. Por outro lado, se utilizarmos um valor de $D$ muito baixo esse algoritmo pode devolver uma política diferente da ótima. Assim, para evitar a necessidade de que um projetista escolha um bom valor para esses parâmetros, pode-se utilizar parâmetros extremos ( $D$ muito alto, ou $\gamma$ muito próximo de um) e utilizar métodos para detecção de becos-sem-saída, que permitam que o valor de becos-sem-saída seja inicializado com o valor $D$, evitando a necessidade de fazer muitas atualizações nesses estados, conforme propomos nesse trabalho de mestrado.

Em geral, todos os algoritmos que lidam com becos-sem-saída podem, de alguma forma, ser beneficiados por um detector de becos-sem-saída. Por exemplo, os algoritmos que computam a probabilidade máxima de alcançar um estado meta poderiam ignorar becos-sem-saída. O próprio MAXPROB MDP poderia ser utilizado para detectar becos-sem-saída (Definição 12). Isto é, basta verificar quais estados tem probabilidade máxima igual a zero de alcançar uma meta.

No próximo capítulo, apresentamos heurística para planejamento e mostramos como elas podem ser utilizadas para detectar certos tipos de becos-sem-saída. No Capítulo 5 apresentamos uma caraterização formal de alguns dos modelos vistos neste capítulo, e, no Capítulo 6 mostramos como essa caracterização pode ser adaptada para criar um método capaz de detectar todos os becos-semsaída de um problema de forma eficiente, e como este método pode ser utilizado por um planejador. 


\section{Capítulo 4}

\section{Heurísticas baseadas em modelos}

Na Seção 2.2, vimos que heurísticas são utilizadas em soluções de planejamento probabilístico para inicializar a função valor dos estados, o que pode reduzir a quantidade de atualizações necessária para a convergência dos algoritmos de programação dinâmica.

Uma heurística é uma função $h: S \rightarrow \mathbb{R}$ que estima o menor custo, ou a menor distância (considerando custos unitários), para alcançar um estado meta $s^{\prime} \in G$ a partir de um estado $s \in S$. Heurísticas são usadas por algoritmos de busca para definir quais estados serão explorados primeiro.

Nos últimos anos pudemos observar um grande avanço na área de planejamento automatizado devido às diversas melhorias nas heurísticas usadas pelos algoritmos de busca dos planejadores determinísticos. Certas heurísticas permitem que algoritmos visitem um número menor de estados durante a busca, sem perderem a garantia de que o problema seja resolvido de forma ótima. Heurísticas também podem ser utilizadas para resolver problemas muito grandes de forma sub-ótima.

Uma heurística pode ser computada a partir de uma versão simplificada do problema, em que algumas características do problema original são relaxadas. Por exemplo, para estimar a menor rota entre dois pontos de um mapa podemos usar a distância euclidiana entre esses pontos, ignorando as restrições que as estradas impõem sobre as possíveis rotas.

Uma heurística $h$ é dita admissivel se ela não superestima o custo ótimo de nenhum estado, isto é, $\forall s \in S: h(s) \leq h^{*}(s)$, sendo $h^{*}(s)$ o menor custo para alcançar a meta a partir do estado $s$. Por exemplo, a distância euclidiana é uma heurística admissível para o problema de encontrar a menor rota entre duas cidades. Dizemos que a heurística $h(s)$ é mais informativa que outra heurística $h^{\prime}(s)$ se $h(s)$ se aproxima mais de $h^{*}(s)$ do que $h^{\prime}(s)$. Em geral, algoritmos de busca heurística, como A*, têm garantia de encontrar uma solução ótima se a heurística utilizada for admissível, no entanto, heurísticas admissíveis podem ser pouco informativas. Abrindo mão de garantias de otimalidade, a busca pode ser mais eficiente com o uso de heurísticas não admissíveis porém mais informativas [Russell e Norvig, 2010].

As heurísticas podem ser projetadas de duas formas: considerando informações específicas do problema, ou, utilizando informações específicas da linguagem em que os problemas são descritos. Portanto, dividimos as heurísticas segundo o tipo de conhecimento do domínio utilizado da seguinte forma:

Heurísticas dependentes de domínio: utilizam informações específicas do problema para estimar a distância de um dado estado $s$ para um estado meta. Geralmente, uma heurística dependente de domínio só é aplicável nos problemas do domínio para o qual ela foi desenvolvida.

Heurísticas independentes de domínio: utilizam informações da linguagem de descrição do problema, por exemplo, a linguagem de ações definida na Seção 2.4 (Capítulo 2), e podem ser aplicadas em diferentes tipos de problema descritos nessa linguagem [Bonet e Geffner, 2001].

Uma forma de definir heurísticas para planejamento probabilístico é estimar o custo para atingir um estado meta em uma versão relaxada determinística do problema [Bonet e Geffner, 2005]. Isto é, dado um problema de planejamento probabilístico $\mathcal{P}_{i}^{\text {prob }}$ e sua versão determinizada $\mathcal{P}_{i}^{\text {det }}$, uma 
estimava do custo esperado de um estado $s$ é dada pelo custo de levar o agente do estado $s$ para um estado meta em $\mathcal{P}_{i}^{\text {det }}$. Em geral, para computar o custo no problema relaxado são utilizadas heurísticas de planejamento clássico.

Seja $\mathcal{P}_{i}^{\text {prob }}=\left\langle\mathbb{P}, \mathbb{A}, s_{0}, \mathbb{G}\right\rangle$ um problema de planejamento probabilístico (Seção 2.4) e $\mathcal{P}_{i}^{\text {det }}=$ $\left\langle\mathbb{P}, \mathbb{A}^{\prime}, s_{0}, \mathbb{G}\right\rangle$ a versão relaxada determinística de $\mathcal{P}_{i}^{\text {prob }}$. O conjunto de ações determinísticas $\mathbb{A}^{\prime}$ pode ser definido de duas formas [Yoon et al., 2007] (há outras formas de determinizar o conjunto de ações, conforme veremos na Seção 4.2.2):

Determinização parcial: para cada ação probabilística cria-se uma ação determinística. Dada uma ação probabilística $a_{i} \in \mathbb{A}$, em que $a_{i}=\left\langle\right.$ Precond, $\left.\left[p_{1}:\left(A d d_{1}, \operatorname{Del}_{1}\right), \cdots, p_{n}:\left(A d d_{n}, D_{e l}\right)\right]\right\rangle$, sua versão determinizada é dada por $\operatorname{det}\left(a_{1}\right)=\left\langle\right.$ Precond, $\left.A d d_{j}, \operatorname{Del}_{j}\right\rangle$ sendo que $\left(A d d_{j}, D_{e l}\right)$ é o efeito mais provável da ação $a_{i}$. Assim, $\mathbb{A}^{\prime}=\left\{a_{i}^{\prime} \mid a_{i}^{\prime}=\operatorname{det}\left(a_{i}\right), \forall a_{i} \in \mathbb{A}\right\}$.

Determinização total: considera todos os efeitos de uma ação, criando uma ação diferente para cada efeito probabilístico. Dada uma ação probabilística $a_{i} \in \mathbb{A}$, em que $a_{i}=\left\langle\right.$ Precond, $\left[p_{1}\right.$ : $\left.\left.\left(A d d_{1}, D e l_{1}\right), \cdots, p_{n}:\left(A d d_{n}, D_{e l}\right)\right]\right\rangle$, a determinização total de $a_{i}$ cria um conjunto de ações determinísticas $\operatorname{det}\left(a_{i}^{\prime}\right)=\left\{a_{i j}=\left\langle\right.\right.$ Precond Add $d_{j}$, Del $\left.\left._{j}\right\rangle \mid 1 \leq j \leq n\right\}$. Assim, o conjunto de ações relaxadas de $\mathbb{A}$ é dado por $\mathbb{A}^{\prime}=\bigcup \operatorname{det}\left(a_{i}\right), \forall a_{i} \in \mathbb{A}$.

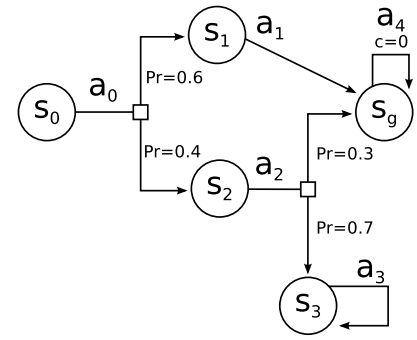

(a)

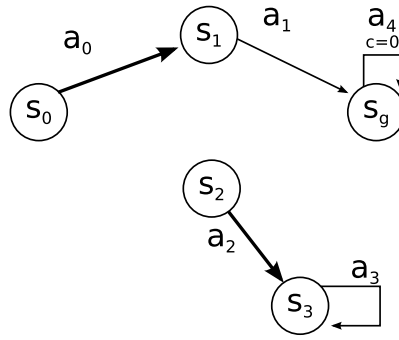

(b)

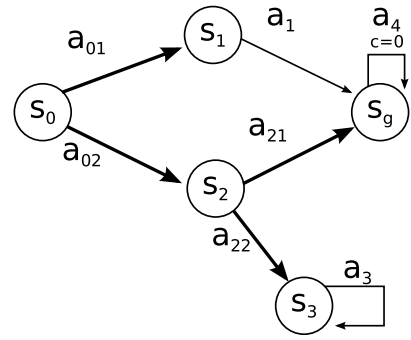

(c)

Figura 4.1: Exemplos de determinização. (a) GMDP $\mathcal{M}\left(\mathcal{P}_{i}^{\text {prob }}\right)$; (b) determinização parcial $\mathcal{M}\left(\mathcal{P}_{i}^{\text {det-p }}\right)$ (escolhendo apenas efeitos mais prováveis); e (c) determinização total $\mathcal{M}\left(\mathcal{P}_{i}^{\text {det-t }}\right)$. (Linhas escuras indicam ações que foram relaxadas.)

A Figura 4.1(a) mostra um GMDP $\mathcal{M}\left(\mathcal{P}_{i}^{\text {prob }}\right)=\left\langle S, A, P, C, s_{0}, G\right\rangle . \mathcal{M}\left(\mathcal{P}_{i}^{\text {det-p }}\right)=\left\langle S, A^{\prime}, T^{\prime}, C^{\prime}, s_{0}, G\right\rangle$ (Figura 4.1(b)) foi gerado pela determinização parcial de $\mathcal{M}(\mathcal{P})_{i}^{\text {prob }}$ obtendo um problema de planejamento determinístico, criando uma ação determinística para a ação $a_{0}$ que leva o agente do estado $s_{0}$ para o estado $s_{1}$, ignorando o efeito que o levaria para $s_{2}$. Note que a determinização parcial pode criar novos becos-sem-saída, por exemplo, o estado $s_{2}$ tornou-se um beco-sem-saída ao descartarmos o efeito que tinha probabilidade 0.3 de alcançar o estado meta $s_{g}$. A Figura 4.1(c) mostra a determinização total de $\mathcal{M}\left(\mathcal{P}_{i}^{\text {prob }}\right), \mathcal{M}\left(\mathcal{P}_{i}^{\text {det-t }}\right)=\left\langle S, A^{\prime \prime}, T^{\prime \prime}, C^{\prime \prime}, s_{0}, G\right\rangle$, em que foram criadas duas ações determinísticas para $a_{0}$ : a ação $a_{01}$ e a ação $a_{02}$.

Proposição 6. Seja $\mathcal{M}\left(\mathcal{P}_{i}^{\text {prob }}\right)=\left\langle S, A, P, C, G, s_{0}\right\rangle$ um GMDP, e $\mathcal{M}\left(\mathcal{P}_{i}^{\text {det-t }}\right)=\left\langle S, A^{\prime}, T^{\prime}, C^{\prime}, s_{0}, G\right\rangle$, a determinização total de $\mathcal{M}\left(\mathcal{P}_{i}^{\text {prob }}\right)$. O estado $s \in S$ é um beco-sem-saída em $\mathcal{M}\left(\mathcal{P}_{i}^{\text {prob }}\right)$, se e somente se, s é um beco-sem-saída em $\mathcal{M}\left(\mathcal{P}_{i}^{\text {det-t }}\right)$.

A Proposição 6 mostra que podemos usar a versão determinizada total de um problema de planejamento probabilístico para identificar becos-sem-saída. A seguir, apresentamos uma descrição de algumas das heurísticas criadas para planejamento determinístico que também são utilizadas em planejamento probabilístico para inicializar a função valor e recochecer becos-semsaída. Em seguida, apresentamos algumas heurísticas especificamente projetadas para planejamento probabilístico [Bonet e Geffner, 2005; Keyder e Geffner, 2008]. Finalmente, descrevemos heurísticas propostas recentemente [Bäckström et al., 2013; Hoffmann et al., 2014; Kolobov et al., 2010; Lipovetzky et al., 2016; Steinmetz et al., 2016], especialmente projetadas para detecção de becossem-saída. 


\subsection{Heurísticas para planejamento determinístico}

Como vimos na introdução desse capítulo, heurísticas podem ser computadas em uma versão relaxada do problema, sendo que, cada versão relaxada explora uma característica específica do problema. Heurísticas para planejamento determinístico também relaxam o problema de várias formas, porém, com base na linguagem de descrição de ações (Seção 2.4). Por exemplo, dado um problema de planejamento determinístico $\mathcal{P}^{\text {det }}=\left\langle\mathbb{P}, \mathbb{A}, s_{0}, \mathbb{G}\right\rangle$ (Definição 8) uma relaxação comum é feita ignorando os efeitos negativos das ações, obtendo assim, um problema $\mathcal{P}^{+}=\left\langle\mathbb{P}, \mathbb{A}^{+}, s_{0}, \mathbb{G}\right\rangle$ em que, $\mathbb{A}^{+}=\{\langle$Precond, Add, $\varnothing\rangle \mid\langle$ Precond, Add,Del $\rangle \in \mathbb{A}\}$. Nesse caso, assumimos que, se uma proposição se torna verdadeira, esta permanecerá verdadeira até o fim da execução do plano, uma vez que nenhuma ação poderá negá-la. Isso garante que, caso uma solução para $\mathcal{P}^{d e t}$ exista, ela será encontrada em no máximo $\left|\mathbb{A}^{+}\right|$passos na versão relaxada $\mathcal{P}^{+}$. Há outras formas de relaxar um problema de planejamento, por exemplo, podemos considerar apenas o custo de alcançar um subconjunto de proposições, ou podemos ignorar o custo de certas proposições.

\subsubsection{Heurísticas $h_{a d d}$ e $h_{\max }$}

As heurísticas propostas pelo planejador de busca heurística HSP (Heuristic Search Planner) [Bonet e Geffner, 2001], são computadas a partir da versão relaxada de um problema que ignora os efeitos negativos das ações, $\mathcal{P}^{+}=\left\langle\mathbb{P}, \mathbb{A}^{+}, s_{0}, \mathbb{G}\right\rangle$. Essas heurísticas estimam o custo para tornar um conjunto de proposições $\Delta \subseteq \mathbb{P}$ verdadeiras partindo de um estado $s \in S$, em função do custo de se alcançar cada proposição $p \in \Delta$ de maneira independente. $\mathrm{O}$ conjunto $\Delta$ pode representar a meta ou a precondição de uma ação.

O custo de tornar uma proposição $p \in \mathbb{P}$ verdadeira a partir do estado $s$, denotado por $c_{s}(p)$, é definido recursivamente como:

$$
c_{s}(p)= \begin{cases}0 & \text { se } p \in s \\ \infty & \text { se } \forall a \in \mathbb{A}^{+}: p \notin \operatorname{Add}(a) \\ \min _{a \in \mathbb{A}^{+} \mid p \in \operatorname{Add}(a)} C(a)+c_{s}(\operatorname{Precond}(a)) & \text { se } \exists a \in \mathbb{A}^{+}: p \in \operatorname{Add}(a)\end{cases}
$$

Um método para calcular $c_{s}(p)$ é o encadeamento para frente (forward chaining). Sendo o valor inicial de $c_{s}(p)$ zero, se $p \in s$, e infinito, caso contrário, $c_{s}(p)$ é atualizado considerando todas as ações que adicionam $p$ da seguinte forma:

$$
c_{s}(p) \leftarrow \min \left[c_{s}(p), C(a)+c_{s}(\operatorname{Precond}(a))\right] .
$$

Isto é, para uma dada ação $a, c_{s}(p)$ é atualizado com o mínimo entre o seu valor atual e a soma do custo da ação $a, C(a)$, com o custo de alcançar as precondições de $a$ a partir do estado $s$, $(c(\operatorname{Precond}(a)))$. Observamos que essa atualização depende da computação do custo de um conjunto de átomos $\Delta=\operatorname{Precond}(a) \subseteq \mathbb{P}$. Há duas formas de fazer esse cálculo e cada uma cria uma heurística diferente.

Podemos computar o custo para alcançar um conjunto de átomos $\Delta \subseteq \mathbb{P}$ utilizando a soma entre os custos de cada átomo $p \in \Delta$, isto é:

$$
c_{s}(\Delta)=\sum_{p \in \Delta} c_{s}(p)
$$

Nesse caso, obtemos a heurística $h_{a d d}(s)=c_{s}(\mathbb{G})=\sum_{p \in \mathbb{G}} c_{s}(p)$.

Também podemos computar o custo de se alcançar o conjunto de átomos $\Delta \subseteq \mathbb{P}$ considerando o valor máximo dos custos de se alcançar cada átomo $p \in \Delta$, isto é:

$$
c_{s}(\Delta)=\max _{p \in \Delta} c_{s}(p)
$$

Nesse caso, obtemos a heurística $h_{\max }(s)=c_{s}(\mathbb{G})=\max _{p \in \mathbb{G}} c_{s}(p)$. 
Note que a heurística $h_{a d d}$ é muito pessimista por considerar que cada átomo será alcançado por planos independentes, tornando-se uma heurística não admissível [Bylander, 1994]. Por exemplo, considere um problema com uma ação $a_{1}=\langle\{p\},\{q, r\}, \varnothing\rangle$ com custo unitário, cujo estado inicial é $s_{0}=\{p\}$ e a meta é $\mathbb{G}=\{p, q, r\}$. Nesse problema $h^{*}\left(s_{0}\right)=1$, já que só é necessário aplicar a ação $a_{1}$ uma vez para adicionar as duas submetas $q$ e $r$. Nesse exemplo, temos $h_{\text {add }}\left(s_{0}\right)=c_{s_{0}}(\mathbb{G})=$ $c_{s}(p)+c_{s}(q)+c_{s}(r)=0+1+1=2$. Observamos que a heurística $h_{\text {add }}$ não leva em conta que ao adicionar uma das submetas a outra também pode ser adicionada indiretamente e, portanto, ela computa um custo maior que o necessário, o que a torna não admissível.

A heurística $h_{\max }$ é admissível, no entanto, é uma heurística pouco informativa por ser muito otimista. Observe que essa heurística assume que ao adicionar o átomo mais caro do conjunto de submetas $\Delta \subseteq \mathbb{P}$, os outros átomos também são adicionados indiretamente. Isto é, $h_{\max }$ assume que o custo para atingir um conjunto de proposições é dado apenas pelo custo da proposição mais cara, desprezando o custo das demais proposições.

\subsubsection{Heurística $h_{F F}$}

A heurística $h_{F F}$ é usada pelo planejador clássico FF (Fast Forward) [Hoffmann, 2001]. Assim como as heurísticas do HSP, essa heurística também é computada a partir de um versão relaxada do problema $\mathcal{P}^{+}$, que desconsidera o conjunto de efeitos negativos das ações. Porém, a heurística $h_{F F}$ não assume a independência total das proposições, sendo capaz de reconhecer quando uma ação pode adicionar mais de uma proposição. O seu cálculo é baseado no planejador determinístico GraphPlan [Blum e Furst, 1997], descrito brevemente a seguir.

Graphplan. O Graphplan [Blum e Furst, 1997] se divide em duas fases. Na primeira, é construído um grafo de planejamento e na segunda, o grafo é usado para a extração de um plano solução, se existir um. A construção do grafo de planejamento é feita intercalando camadas de literais (proposições atômicas positivas e negativas) e de ações: a primeira camada contém todos os literais do estado inicial $s_{0}$; a segunda camada contém todas as ações aplicáveis em $s_{0}$ sendo que cada ação é ligada aos literais da sua precondição na camada anterior; em seguida, uma nova camada é criada contendo todos os literais da primeira camada mais os literais dos efeitos das ações da camada anterior, adicionando-se as ligações entre ações e seus respectivos efeitos. Em cada camada, ações em conflito (que não poderiam ser executadas em nenhuma ordem total sem que uma interaja com a outra) são marcadas como mutex (mutual exclusion). Literais de uma mesma camada também podem ser marcados como mutexes. O grafo é expandido até atingir uma camada em que todos os literais da meta $(\mathbb{G})$ sejam verdadeiros sem marcação de mutex, ou até que duas camadas de literais se repitam (ponto fixo), caso em que não existe uma solução. Finalmente, na segunda fase do GraphPlan, um plano solução é extraído fazendo-se uma busca regressiva a partir da última camada do grafo, escolhendo-se uma ação para cada literal (ou conjunto de literais) em $\mathbb{G}$ e em Precond(a), que não estejam em mutex. Como exemplo de mutex entre ações de uma mesma camada, considere uma ação $a_{1}$ com precondição $p$ e outra ação $a_{2}$ com efeito negativo $\neg p$, isto é, $p \in \operatorname{Precond}\left(a_{1}\right) \mathrm{e}$ $p \in \operatorname{Del}\left(a_{2}\right)$. Ao se selecionar as ações $a_{1}$ e $a_{2}$ em um plano, elas conflitam entre si. Um exemplo trivial de mutex entre literais de mesma camada é $p$ e $\neg p$; ou dois literais $p$ e $q$ que só poderiam ser verdadeiros escolhendo-se duas ações da camada anterior que estão em mutex.

A heurística $h_{F F}$ [Hoffmann, 2001] constrói um grafo de planejamento relaxado a partir de um estado, ignorando os efeitos negativos das ações e devolve como estimativa o custo do plano extraído desse grafo. Se, durante a construção do grafo, a meta não for alcançada, a heurística retorna o valor infinito.

Formalmente, seja $\mathcal{P}^{\text {det }}=\left\langle\mathbb{P}, \mathbb{A}, C, s_{0}, \mathbb{G}\right\rangle$ um problema de planejamento determinístico e $\mathcal{M}\left(\mathcal{P}^{\text {det }}\right)=$ $\left\langle S, A, T, C, s_{0}, G\right\rangle$ o respectivo modelo de estados. Dado um estado $s \in S$, temos que o valor de $h_{F F}(s)$ é o tamanho do plano solução (quantidade de ações do plano) obtido pelo Graphplan a partir do problema relaxado $\mathcal{P}^{+}=\left\langle\mathbb{P}, \mathbb{A}^{+}, C, s, \mathbb{G}\right\rangle$, em que $\mathbb{A}^{+}$é o conjunto de ações sem efeitos negativos e $s$ é o novo estado inicial. Observe que, sem efeitos negativos, não há conflitos entre ações ou literais 
positivos, logo não é necessário computar o conjunto de mutexes, o que facilita a construção do grafo e a extração de um plano.

Como mencionado anteriormente, apesar de desconsiderar os efeitos negativos das ações, a heurística $h_{F F}$ não assume a independência entre as proposições de uma camada, uma vez que, durante a extração do plano, uma mesma ação pode ser selecionada para um ou mais efeitos. Seja $\mathcal{P}^{\text {det }}=\left\langle\mathbb{P}, \mathbb{A}, C, s_{0}, \mathbb{G}\right\rangle$ um problema de planejamento determinístico com duas ações com custo unitário, $a_{p}=\langle\{p\},\{r\},\{p\}\rangle$ e $a_{q}=\langle\{q\},\{s, t\}, \varnothing\rangle$, cujo estado inicial é $s_{0}=\{p, q\}$ e a meta é $\mathbb{G}=\{r, s, t\}$. A solução seria um dos planos de tamanho dois: $\left\langle a_{p}, a_{q}\right\rangle$ ou $\left\langle a_{q}, a_{p}\right\rangle, \operatorname{logo} h^{*}\left(s_{0}\right)=2$. A Figura 4.2 mostra como seria o grafo de planejamento relaxado utilizado para computar $h_{F F}\left(s_{0}\right)$ nesse problema. A primeira camada de literais $L_{0}$ só contém as proposições verdadeiras no estado inicial $s_{0}$. A camada de ações $A_{0}$ contém ambas as ações $a_{p}$ e $a_{q}$, uma vez que a precondição de ambas é satisfeita em $L_{0}$. A camada $L_{1}$ contém os literais da camada anterior $p$ e $q$ (adicionados por uma ação trivial de persistência) e os literais $r, s$ e $t$, adicionados pelas ações $a_{p}$ e $a_{q}$. Note que apesar da ação $a_{p}$ remover o literal $p$ o grafo relaxado desconsidera isso e não cria um mutex entre os literais $p$ e $r$ na camada $L_{1}$. Finalmente, a camada $L_{1}$ contém todos os literais da meta $\mathbb{G}$, e assim, a expansão do grafo termina. Durante a extração do plano, as ações $a_{p}$ e $a_{q}$ são selecionadas, obtendo $h_{F F}\left(s_{0}\right)=C\left(a_{p}\right)+C\left(a_{q}\right)=2=h^{*}\left(s_{0}\right)$.

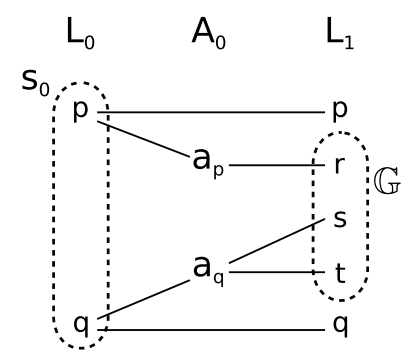

Figura 4.2: Grafo de planejamento relaxado. $L_{i}$ é a camada de literais i e $A_{i}$ é a camada de ações $i$. As linhas pontilhadas na camada inicial $L_{0}$ indicam as proposições do estado inicial $s_{0}$ e na camada $L_{1}$, indicam as proposições da meta $\mathbb{G}$. O plano solução é $\left\langle a_{p}, a_{q}\right\rangle$ ou $\left\langle a_{q}, a_{p}\right\rangle$.

No exemplo da Figura 4.2, podemos observar algumas diferenças entre a heurística $h_{F F}$ e as heurísticas $h_{a d d}$ e $h_{\max }$. Como $h_{\max }$ só considera o custo de uma proposição da meta, nesse problema temos que $h_{\max }\left(s_{0}\right)=1$, mostrando que essa heurística é pouco informativa quando comparada a $h_{F F}$ para esse exemplo. Por outro lado, $h_{a d d}$ não percebe que $a_{q}$ adiciona duas proposições da meta e soma o custo dessa ação duas vezes, $\operatorname{assim} h_{a d d}\left(s_{0}\right)=3$, o que a torna não admissível para esse problema.

Por ignorar os efeitos negativos das ações, as heurísticas computadas sobre $\mathcal{P}^{+}$podem ser pouco informativas. Por exemplo, seja $\mathcal{P}^{\text {det }}=\left\langle\mathbb{P}, \mathbb{A}, C, s_{0}, \mathbb{G}\right\rangle$ em que $\mathbb{A}$ tem duas ações $a_{p}=\langle\varnothing,\{p\}, \varnothing\rangle$ e $a_{q}=\langle\{p\},\{q\},\{p\}\rangle$; o estado inicial é $s_{0}=\varnothing$; e a meta $\mathbb{G}=\{p, q\}$. Observe que a ação original $a_{q}$ torna a proposição $p$ falsa e dessa forma, seria necessário aplicar duas vezes a ação $a_{p}$ para alcançar a meta $\mathbb{G}$, assim, $h^{*}\left(s_{0}\right)=3$. A versão relaxada é $\mathcal{P}^{+}=\left\langle\mathbb{P}, \mathbb{A}^{+}, C, s_{0}, \mathbb{G}\right\rangle$ sendo $\mathbb{A}^{+}$composto pelas ações relaxadas $a_{p}^{\prime}=\langle\varnothing,\{p\}, \varnothing\rangle$ e $a_{q}^{\prime}=\langle\{p\},\{q\}, \varnothing\rangle$. Nesse problema, como a versão relaxada ignora os efeitos negativos, temos que $h_{\text {add }}\left(s_{0}\right)=c_{s_{0}}(\mathbb{G})=c_{s}(p)+c_{s}(q)=1+1=2$. Nas próximas seções apresentamos heurísticas que relaxam outros aspectos do problema de planejamento.

\subsubsection{Heurística $h_{m}$}

A heurística $h_{m}$ [Haslum e Geffner, 2000] é admissível e, assim como as heurísticas anteriores, ela é computada no espaço de átomos, isto é, ela é uma função dos custos dos átomos do problema, no entanto, ela não ignora os efeitos negativos das ações. Ao invés de computar o custo para alcançar os átomos de um conjunto de proposições de forma independente, a heurística $h_{m}$ computa o custo para alcançar subconjuntos de proposições com uma cardinalidade limitada $m \geq 1$. Seja $\mathcal{P}^{\text {det }}=$ $\left\langle\mathbb{P}, \mathbb{A}, C, s_{0}, \mathbb{G}\right\rangle$ um problema de planejamento determinístico. Dado um conjunto de proposições 
$\Delta \subseteq \mathbb{P}$ a serem alcançados a partir do estado $s \in S$, a heurística $h_{m}$ é definida recursivamente:

$$
h_{m}(s, \Delta)= \begin{cases}0 & \text { se } \Delta \subseteq s, \\ \infty & \text { se } \forall a \in \mathbb{A}: R(\Delta, a)=\perp, \\ \min _{a \in \mathbb{A}}\left[h_{m}(s, R(\Delta, a))+C(a)\right] & \text { se }|\Delta| \leq m, \\ \max _{\Delta^{\prime} \subseteq \Delta,\left|\Delta^{\prime}\right| \leq m} h_{m}\left(s, \Delta^{\prime}\right) & \text { se }|\Delta|>m,\end{cases}
$$

sendo $R(\Delta, a)$, a operação de regressão de um conjunto de proposições $\Delta \subseteq \mathbb{P}$ por uma ação $a \in \mathbb{A}$, definida como:

$$
R(\Delta, a)= \begin{cases}(\Delta \backslash \operatorname{Add}(a)) \cup \operatorname{Precond}(a) & \text { se } \operatorname{Add}(a) \subseteq \Delta \text { e } \operatorname{Del}(a) \cap \Delta=\varnothing \\ \perp & \text { caso contrário. }\end{cases}
$$

Intuitivamente, a Equação 4.1 diz que se todas as proposições de $\Delta$ já forem verdadeiras no estado $s$ então o custo de $\Delta$ é nulo; se nenhuma ação puder adicionar algum átomo de $\Delta$, então é impossível alcançar $\Delta$ a partir de $s$, e o custo de $\Delta$ é infinito; se $\Delta$ tiver até $m$ proposições então o custo é definido recursivamente pela ação de menor custo relevante para $\Delta$ segundo a regressão de $\Delta$ (Equação 4.2); e se $\Delta$ tiver mais que $m$ proposições o custo é definido recursivamente em função do conjunto de proposições de maior custo com até $m$ átomos.

Assim, o custo para alcançar a meta $\mathbb{G}$ a partir do estado $s \subseteq \mathbb{P}$, segundo a heurística $h_{m}$, é dado por:

$$
h_{m}(s)=h_{m}(s, \mathbb{G}) \text {. }
$$

Observamos então que $h_{m}$ define uma família de heurísticas, sendo que para cada $m>0$ obtémse uma heurística diferente. Assim, para $m=1$, obtemos a heurística $h_{1}$ que é igual à heurística $h_{\max }$, pois o custo de atingir um conjunto de átomos é estimado a partir de conjuntos de tamanho unitário. Para $m=2$, obtemos a heurística $h_{2}$ que é equivalente ao tamanho do plano gerado pelo planejador GraphPlan, uma vez que computar $h_{2}$ (que considera pares de proposições) está diretamente relacionado à ideia de levar em consideração os mutexes entre literais.

Uma generalização da heurística $h_{m}$ é a $h_{\mathcal{C}}$, em que $\mathcal{C} \subseteq 2^{\mathbb{P}}$ é um conjunto de conjuntos arbitrários de proposições [Keyder et al., 2014]. A heurística $h_{\mathcal{C}}$ só considera o custo máximo de atingir os conjuntos de $\mathcal{C}$ :

$$
h_{\mathcal{C}}(s, \Delta)= \begin{cases}0 & \text { se } \Delta \subseteq s, \\ \infty & \text { se } \forall a \in A: R(\Delta, a)=\perp, \\ \min _{a \in \mathbb{A}}\left[h_{\mathcal{C}}(s, R(\Delta, a))+C(a)\right] & \text { se } \Delta \in \mathcal{C}, \\ \max _{\Delta^{\prime} \subseteq \Delta, \Delta^{\prime} \in \mathcal{C}} h_{\mathcal{C}}\left(s, \Delta^{\prime}\right) & \text { se } \Delta \notin \mathcal{C} .\end{cases}
$$

$\mathrm{O}$ conjunto $\mathcal{C}$ pode ser escolhido livremente. Note que, quando $\mathcal{C}$ possui todos os conjuntos com $m$ ou menos proposições, então $h_{\mathcal{C}}$ é igual a $h_{m}$.

\subsubsection{Heurística $p d b$}

A heurística pattern database $(p d b)$ é baseada no conceito de padrão. Dado um padrão $\mathcal{Y} \subseteq \mathbb{P}$, um subconjunto das proposições atômicas do problema, a heurística pdb cria uma projeção do espaço de estados que ignora as proposições que não fazem parte de $\mathcal{Y}$ [Haslum et al., 2007]. Assim, a heurística $p d b$ relaxa o problema ignorando parte das proposições, criando assim um espaço de estados abstrato pequeno o bastante para ser resolvido por uma busca não informada (exaustiva).

Seja $\mathcal{P}^{\text {det }}=\left\langle\mathbb{P}, \mathbb{A}, C, s_{0}, \mathbb{G}\right\rangle$ um problema de planejamento determinístico e $\mathcal{Y} \subseteq \mathbb{P}$ um padrão. A versão relaxada de $\mathcal{P}^{\text {det }}$, que só considera as proposições de $\mathcal{Y}$, é dada por $\mathcal{P}^{\mathcal{Y}}=\left\langle\mathcal{Y}, \mathbb{A}^{\mathcal{Y}}, C, s_{0}^{\mathcal{Y}}, \mathbb{G}^{\mathcal{Y}}\right\rangle$, em que:

- $\mathbb{A}^{\mathcal{Y}}=\left\{a^{\prime} \mid a^{\prime}=\langle\operatorname{Precond}(a) \cap \mathcal{Y}, \operatorname{Add}(a) \cap \mathcal{Y}, \operatorname{Del}(a) \cap \mathcal{Y}\rangle, \forall a \in \mathbb{A}\right\}$ 
- $s_{0}^{\mathcal{Y}}=s_{0} \cap \mathcal{Y} ; \mathrm{e}$

- $\mathbb{G}^{\mathcal{Y}}=\mathbb{G} \cap \mathcal{Y}$.

Assim, seja $\mathcal{P}^{\mathcal{Y}}=\left\langle\mathbb{P}, \mathbb{A}, C, s_{0}, \mathbb{G}\right\rangle$ um problema de planejamento determinístico, denotamos por $\mathcal{M}\left(\mathcal{P}^{\mathcal{Y}}\right)=\left\langle S^{\mathcal{Y}}, A^{\mathcal{Y}}, T^{\mathcal{Y}}, s_{0}^{\mathcal{Y}}, G^{\mathcal{Y}}\right\rangle$ o respectivo modelo de transição de estados (a Seção 2.4 mostra como extrair o modelo de transição de estados $\mathcal{M}\left(\mathcal{P}^{\text {det }}\right)$ de um problema de planejamento determinístico $\left.\mathcal{P}^{\text {det }}\right)$.

Geralmente, a heurística pattern database é definida para problemas com um conjunto de variáveis multi-valoradas $\mathcal{V}=\left\{v_{1}, v_{2}, \cdots, v_{n}\right\}$, uma extensão dos problemas de planejamento determinístico (Definição 9), em que cada variável $v_{i}$ pode assumir como valor um elemento de um conjunto discreto de valores, definido pelo domínio de $v_{i}: \operatorname{dom}\left(v_{i}\right)=\left\{d_{1}, d_{2}, \cdots, d_{m}\right\}$. Nesse caso, um estado é representado por uma valoração de todas as variáveis, a meta é descrita por uma valoração parcial e as ações são definidas por valorações parciais que alteram o valor das variáveis de domínio. Dado um problema de planejamento determinístico, definido sobre variáveis proposicionais, é possível extrair automaticamente uma versão multi-valorada deste problema [Edelkamp, 2001]. Por exemplo, em um domínio em que um robô carrega objetos de uma sala para outra, uma variável multi-valorada poderia ser a localização do objeto, que pode estar em uma das salas ou nas mãos do robô, observe que em todo estado uma dessas proposições é verdadeira, mas nunca duas ao mesmo tempo.

Em um problema com variáveis multi-valoradas um padrão é um subconjunto das variáveis de domínio, isto é, $\mathcal{Y} \subseteq \mathcal{V}$. Assim, dado um padrão $\mathcal{Y} \subseteq \mathcal{V}$ é criado um espaço de estados abstratos $S^{\mathcal{Y}}$, em que cada estado agrupa todos os estados originais nos quais os valores das variáveis contidas em $\mathcal{Y}$ são iguais. Dado um estado abstrato $s^{\prime} \in S^{\mathcal{Y}}$ e um estado $s \in S$, se $s$ e $s^{\prime}$ atribuem os mesmos valores para todas as variáveis de $\mathcal{Y}$ dizemos que $s$ pertence a $s^{\prime}\left(s \in s^{\prime}\right)$ e que $s^{\prime}$ agrega $s$.

A Figura 4.3 mostra um exemplo de como podemos fazer essa projeção. Seja $\mathcal{V}=\left\{v_{1}, v_{2}\right\}$, $\operatorname{dom}\left(v_{1}\right)=(1,3)$ e $\operatorname{dom}\left(d_{2}\right)=(1,4)$ temos que $S=\left\{s_{11}=\left\{v_{1}=1, v_{2}=1\right\}, s_{12}=\left\{v_{1}=1, v_{2}=\right.\right.$ $\left.2\}, \cdots, s_{34}=\left\{v_{1}=3, v_{2}=4\right\}\right\}$. O padrão $\mathcal{Y}_{1}=\left\{v_{1}\right\}$ cria um espaço de estados com apenas três estados abstratos $S^{\mathcal{Y}_{1}}=\left\{s_{1}^{\prime}=\left\{v_{1}=1\right\}, s_{2}^{\prime}=\left\{v_{1}=2\right\}, s_{3}^{\prime}=\left\{v_{1}=3\right\}\right\}$. De forma análoga, o padrão $\mathcal{Y}_{2}=\left\{v_{2}\right\}$ cria o espaço de estados abstratos $S^{\mathcal{Y}_{2}}=\left\{s_{1}^{\prime}=\left\{v_{2}=1\right\}, s_{2}^{\prime}=\left\{v_{2}=2\right\}, s_{3}^{\prime}=\left\{v_{2}=3\right\}, s_{4}^{\prime}=\left\{v_{2}=4\right\}\right\}$. Assim, observamos que os estados do problema original $s_{11}=\left\{v_{1}=1, v_{2}=1\right\}, s_{12}=\left\{v_{1}=1, v_{2}=\right.$ $2\}, \cdots, s_{14}=\left\{v_{1}=1, v_{2}=4\right\}$ são aglomerados no estado $s_{1}^{\prime}$ do espaço abstrato $S^{\mathcal{Y}_{1}}$.

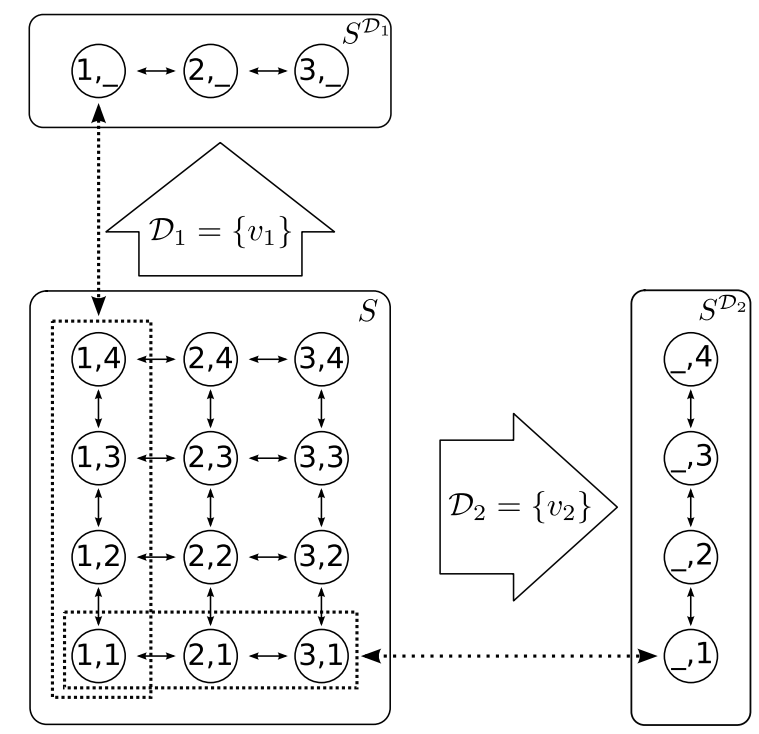

Figura 4.3: Exemplos de projeções sobre os padrões $\mathcal{Y}_{1}$ e $\mathcal{Y}_{2}$. Cada estado é dado por um par que representa a valoração das variáveis multi-valoradas $v_{1}$ e $v_{2}$. O símbolo _ indica que a variável não é definida no estado abstrato. Os retângulos pontilhados indicam os estados que foram agregados em uma projeção. As setas duplamente direcionadas indicam a relação de um estado abstrato e seus respectivos estados concretos. 
Para cada padrão $\mathcal{Y} \subseteq \mathbb{P}$, cria-se uma heurística pattern database diferente denotada por $h \mathcal{Y}$. A computação de uma heurística $h \mathcal{Y}$ é realizada antes da fase de planejamento, para isso, o custo ótimo $h^{*}\left(s^{\prime}\right)$ de cada estado de $s^{\prime} \in S^{\mathcal{Y}}$ é calculado e armazenado. Assim, durante a fase de planejamento, a heurística para os estados contidos em um estado real $s \in S$ é definida de acordo com o custo ótimo do respectivo estado abstrato, isto é, $\forall s \in s^{\prime}=h_{\mathcal{Y}}(s)=h^{*}\left(s^{\prime}\right)$. No exemplo da Figura 4.3 supondo que a meta seja $\mathbb{G}=\left\{v_{1}=3, v_{2}=4\right\}$, dado o padrão $\mathcal{Y}_{1}=\left\{v_{1}\right\}$ temos que a meta no espaço abstrato é $\mathbb{G}^{\prime}=\{v 1=3\}$ satisfeita pelo estado abstrato $s_{3}^{\prime}=\{v 1=3\}$, logo, $h_{\mathcal{Y}_{1}}\left(\left\{v_{1}=1, v_{2}=1\right\}\right)=$ $h_{\mathcal{Y}_{1}}\left(\left\{v_{1}=1, v_{2}=2\right\}\right)=h_{\mathcal{Y}_{1}}\left(\left\{v_{1}=1, v_{2}=3\right\}\right)=h_{\mathcal{Y}_{1}}\left(\left\{v_{1}=1, v_{2}=4\right\}\right)=h_{\mathcal{Y}_{1}}^{*}\left(\left\{v_{1}=1\right\}\right)=2$. De forma semelhante, se usarmos o padrão $\mathcal{Y}_{2}=\left\{v_{2}\right\}$, então temos $h_{\mathcal{Y}_{2}}\left(\left\{v_{1}=1, v_{2}=1\right\}\right)=h_{\mathcal{Y}_{2}}\left(\left\{v_{1}=2, v_{2}=\right.\right.$ $1\})=h_{\mathcal{Y}_{2}}\left(\left\{v_{1}=3, v_{2}=1\right\}\right)=h_{\mathcal{Y}_{2}}^{*}\left(\left\{v_{2}=1\right\}\right)=3$.

Duas heurísticas definidas em função de padrões diferentes $h_{\mathcal{Y}_{1}}(s)$ e $h_{\mathcal{Y}_{2}}(s)$, podem ser combinadas utilizando o máximo ou a soma das mesmas. O máximo entre duas heurísticas admissíveis cria uma nova heurística admissível. A combinação de heurísticas pela soma também pode gerar uma nova heurística admissível se certas condições forem satisfeitas, por exemplo, se nenhuma ação altera os valores das variáveis dos dois padrões [Haslum et al., 2005]. Utilizando ainda o exemplo da Figura 4.3 observamos que $h_{\mathcal{Y}_{1}+\mathcal{Y}_{2}}(s)=h_{\mathcal{Y}_{1}}(s)+h_{\mathcal{Y}_{2}}(s)$ é admissível pois nenhuma ação altera o valor de $v_{1}$ e $v_{2}$, por exemplo, $h_{\mathcal{Y}_{1}+\mathcal{Y}_{2}}\left(\left\{v_{1}=1, v_{2}=1\right\}\right)=5$, o que coincide com o custo ótimo.

Haslum et al. [2005] observaram que o meio de extrair as variáveis multi-valoradas definidas por Edelkamp [2001] desconsiderava as precondições das ações, o que podia criar heurísticas muito otimistas em certos casos. Assim, propuseram novas formas de extrair as variáveis multi-valoradas utilizando informações do estado inicial e das ações, obtendo heurísticas mais informativas.

\subsection{Heurísticas para planejamento probabilístico}

A seguir, apresentamos algumas heurísticas que foram desenvolvidas especificamente para lidar com problemas de planejamento probabilístico, estimando o custo esperado ao invés do custo para atingir um estado meta.

\subsubsection{Heurística $h_{m-m}$}

A heurística min-min $\left(h_{m-m}\right)$, diferentemente das anteriores, é computada no espaço de estados. Ela calcula o menor custo acumulado para atingir um estado considerando o efeito de menor custo. A heurística $h_{m-m}$ é definida recursivamente pela seguinte equação [Bonet e Geffner, 2005]:

$$
h_{m-m}(s)=\min _{a \in A(s)}\left\{C(a)+\min _{s^{\prime}: P\left(s^{\prime} \mid s, a\right)>0} h_{m-m}\left(s^{\prime}\right)\right\} .
$$

Sendo que $h_{m-m}(s)=0, \forall s \in G$. Note que essa heurística é muito otimista, pois assume que uma transição probabilística irá para o estado de menor custo. Considerando ações com custo unitário, essa heurística devolve o número mínimo de passos que leva o agente para um estado meta.

A heurística $h_{m-m}$ pode ser computada por um método de encadeamento para frente, utilizando a Equação 4.4 como uma função de atribuição e inicializando $h_{m-m}(s)$ com 0 se $s \in G$ ou $\infty$, caso contrário. No entanto, esse método pode expandir o mesmo número de estados que algoritmos de busca exaustiva. Para evitar esse problema, podemos encontrar um valor aproximado para $h_{m-m}(s)$ fazendo uma busca heurística numa versão relaxada do problema probabilístico com determinização total, partindo do estado inicial $s$. Tal busca, pode ser realizada com algoritmos clássicos de busca heurística, como o A* ou IDA* (Iterative-Deepening $A^{*}$ ) [Russell e Norvig, 2010], utilizando qualquer heurística de planejamento determinístico, como as heurísticas $h_{F F}, h_{a d d}, h_{m}$ e $p d b$ vistas na seção anterior. 


\subsubsection{A heurística do HMDPP}

Outra forma de relaxar um problema probabilístico é a relaxação self-loop, que considera que os efeitos das ações são independentes, sendo que cada efeito ocorre com probabilidade $p_{i}$ e com probabilidade $1-p_{i}$ ele falha e deixa o agente no mesmo estado. O GMDP da Figura 4.4(b) mostra a relaxação self-loop do GMDP da Figura 4.4(a).

Para resolver essa versão relaxada do problema, Keyder e Geffner [2008] observaram que a incerteza poderia ser removida do problema, compilando as probabilidades nos custos de uma ação determinística. Esse processo é chamado determinização self-loop. Dado um problema de planejamento probabilístico $\mathcal{P}_{i}^{\text {prob }}$, a determinização self-loop cria uma versão determinística $\mathcal{P}_{i}^{\text {det }}$ do problema relaxado, cuja solução é igual a da relaxação self-loop. O GMDP da Figura 4.4(c) mostra a determinização self-loop do GMDP da Figura 4.4(a). Assim como a determinização total, a determinização de laço próprio cria uma nova ação determinística para cada efeito da ação, no entanto, o custo da nova ação determinística é definido pelo custo dividido pela probabilidade do efeito original ocorrer:

$$
C\left(a_{i}\right)=\frac{C(a)}{p_{i}} .
$$

Observe que dessa forma quanto menor a probabilidade de um efeito ocorrer maior será o custo da respectiva ação determinística, assim a solução do problema determinizado dá maior preferência aos efeitos de maior probabilidade.

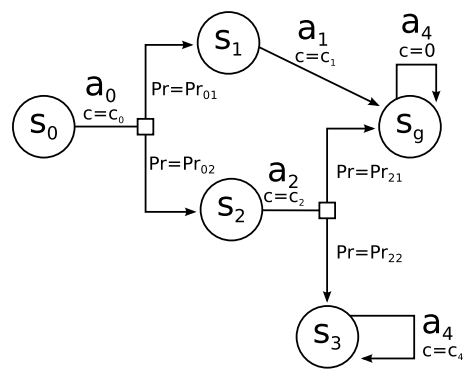

(a)

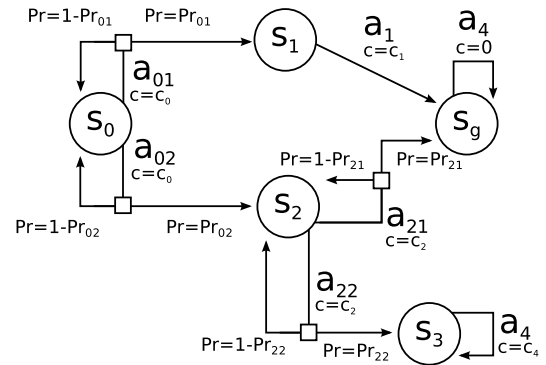

(b)

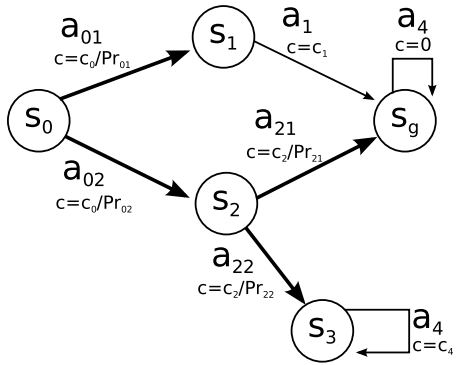

(c)

Figura 4.4: Determinização de laço próprio: (a) GMDP $\mathcal{M}$; (b) relaxação de laço próprio de $\mathcal{M}$; e (c) determinização de laço próprio de $\mathcal{M}$. Linhas escuras indicam ações determinizadas.

O planejador HMDP (HMDPP) [Keyder e Geffner, 2008] é um planejador probabilístico que se baseia em um processo de determinização, assim como o FF-Replan [Yoon et al., 2007]. Esses planejadores criam um plano para a versão relaxada (determinística) e segue esse plano, replanejando caso algum efeito inesperado aconteça. O HMDPP trabalha com a versão relaxada pela determinização de laço próprio, enquanto o FF-Replan usa um planejador clássico para obter um plano sobre o problema relaxado.

O HMDPP computa a heurística $h_{a d d}$ na determinazação self-loop do problema. Apesar da heurística ser muito informativa, ela não é eficiente para a detecção de becos-sem-saída. Assim, o HMDPP também utiliza a heurística $p d b$ para avaliar um estado $s$, que o ajuda a detectar becos-sem-saída, podendo assim criar um plano que evite esses estados. Para combinar as duas heurísticas o HMDPP ranqueia as ações aplicadas em um estado segundo a função

$$
Q_{h}(s, a)=C(a)+\sum_{s^{\prime} \in S} P\left(s^{\prime} \mid s, a\right) h\left(s^{\prime}\right),
$$

em que $h$ é uma função heurística. Assim, o valor esperado de aplicar uma ação $a$ em um estado $s$ é o máximo entre as heurísticas consideradas, isto é, $Q(s, a)=\max \left[Q_{h_{\text {self-loop }}}(s, a), Q_{p d b}(s, a)\right]$.

Entre os problemas com becos-sem-saída da competição de planejamento probabilístico de 2008, o HMDPP foi o planejador que obteve os melhores resultados em relação ao tempo de planejamento probabilístico e qualidade da solução. 


\subsection{Heurísticas para insolubilidade}

As competições internacionais de planejamento focam em problemas que possuem uma solução. Porém, recentemente foi criada uma nova trilha na competição internacional de planejamento que lida com problemas sem solução ${ }^{1}$. Nesta competição, os planejadores recebem um problema e devolvem falha caso não exista uma solução a partir do estado inicial, isto é, caso o estado inicial seja um beco-sem-saída. Tal competição incentiva que os planejadores se tornem mais robustos, permitindo que eles lidem com uma gama maior de problemas.

Considerando que heurísticas são projetadas com intuito de serem mais informativas, o uso destas para lidar com o problema de insolubilidade pode ser ineficiente. Por muito tempo, a tarefa de verificar se um estado pode alcançar um estado meta foi vista como um bônus do problema de estimar a distância para um estado meta, por exemplo, na inicialização da função $V(s)$ por algoritmos de programação dinâmica. Isto é, espera-se que as heurísticas usadas para estimar o custo para alcançar um estado meta devolvam um valor infinito para os estados becos-sem-saída. Assim, trabalhos recentes têm se preocupado em desenvolver métodos dedicados para esta tarefa [Bäckström et al., 2013; Hoffmann et al., 2014; Kolobov et al., 2010; Lipovetzky et al., 2016; Steinmetz e Hoffmann, 2016]. Tais métodos podem ser incorporados aos planejadores para evitar que eles percam muito tempo tentando resolver um problema sem solução, ou, podem ser utilizados diretamente como detectores de becos-sem-saída. Um detector de becos-sem-saída checa se um estado é ou não um beco-sem-saída, e permite que o planejador só calcule a heurística para inicializar a função $V(s)$ de estados que podem alcançar um estado meta. Kolobov et al. [2012b] mostrou que para resolver problemas com becos-sem-saída de forma eficiente, é necessário equipar o planejador com meios para detectá-los.

Os métodos de deteç̧ão de becos-sem-saída trabalham de duas formas: (i) um conjunto de becos-sem-saída são detectados durante a busca e são aplicadas técnicas de aprendizado de máquina para reconhecer outros becos-sem-saída, isto é, estes métodos tentam generalizar o conhecimento de um beco-sem-saída; (ii) é feita uma pré-análise do problema, identificando quais estados são becos-sem-saída antes da busca por uma solução. Essas técnicas exploram alguma estrutura implícita do problema. A seguir apresentamos alguns métodos de cada um desses tipos.

\subsubsection{Aprendendo durante a busca}

Nogoods. Um nogood é uma conjunção de proposições que quando verdadeira em um estado $s$ indicam que $s$ é um beco-sem-saída. O SixthSense [Kolobov et al., 2010] é um meio para aprender nogoods. Este foi o primeiro método dedicado para deteç̧ão de becos-sem-saída aplicado no contexto de problemas de planejamento probabilístico. Esse algoritmo armazena os becos-sem-saída identificados pela busca (estados que não satisfazem a meta e cujos sucessores já foram explorados), depois tenta encontrar os nogoods desses estados utilizando o grafo de planejamento (Seção 4.1.2) e funções base (conjunções que com certeza podem levar para um estado meta). O uso de funções base mostra que essa abordagem está altamente acoplada ao planejador ReTrASE [Kolobov et al., 2012a], por isso, o SixthSense ainda não foi explorado no contexto de outros planejadores ${ }^{2}$.

Cláusulas. Steinmetz e Hoffmann [2016] adaptaram a heurística $h_{\mathcal{C}}$ (Seção 4.1.3) para ser utilizada como um detector de becos-sem-saída. Assim como o SixthSense, utilizam os becos-sem-saída identificados durante a busca para aprender a razão que os impedem de atingir um estado meta. Esse método, inicializa $\mathcal{C}$ com conjuntos de proposição de tamanho um. Ao encontrar um conjunto de becos-sem-saída $\hat{S}$ essa abordagem refina $\mathcal{C}$ adicionando conjuntos de proposições que são inalcançáveis a partir de $\hat{S}$. Finalmente, Steinmetz e Hoffmann [2016] observam que testar se um estado satisfaz cada conjunção em $\mathcal{C}$ pode ser muito custoso computacionalmente, assim desenvolveram um método que aprende disjunções que são testadas de forma mais eficiente.

\footnotetext{
${ }^{1}$ http://unsolve-ipc.eng.unimelb.edu.au/

${ }^{2} \mathrm{O}$ SixthSense não está disponível para testes [Steinmetz et al., 2016].
} 


\title{
4.3.2 Pré-análise
}

Consistência local. Dedicada à análise de insolubilidade, a análise de consistência local [Bäckström et al., 2013] se baseia no fato de que se um estado abstrato $s^{\prime}$ em uma projeção $S^{\mathcal{Y}}$ (espaço de estados abstratos que só considerada as variáveis do padrão $\mathcal{Y} \subseteq \mathcal{V}$, Seção 4.1.4) não pode alcançar a meta então os estados que satisfazem $s^{\prime}$ também não alcançam um estado meta. Assim, foi proposto um algoritmo que, baseado em um parâmetro $k$, verifica se o estado inicial possui solução nas projeções $S^{\mathcal{Y}}$, com $0<|\mathcal{Y}| \leq k$. Se o estado inicial não possuir solução em alguma dessas projeções então o problema original não possui solução. Observamos que esse método pode ser adaptado para detectar becos-sem-saída de forma semelhante à heurística $p d b$. Estados abstratos sem solução podem ser armazenados em uma tabela e, durante a fase de planejamento, estados concretos que pertencem a algum dos estados abstratos sem solução são considerados becos-sem-saída. No entanto, essa técnica é incompleta, sendo limitada pelo tamanho das projeções, isto é, se um estado é um beco sem saída apenas em projeções com tamanho maior que $k$, eles não serão reconhecidos como becos-sem-saída.

\begin{abstract}
Abstrações M\&s [Hoffmann et al., 2014]. Uma abstração é uma versão do espaço de estado, na qual estados semelhantes são agregados fazendo-se um número menor de distinções entre eles. Um exemplo são as abstrações criadas por projeções da heurística pdb (Seção 4.1.4). Abstrações M\&s (Merge and Shrink) são criadas a partir de um processo iterativo de união de variáveis multi-valoradas (duas variáveis se tornam uma cujo domínio é o produto cartesiano dos domínios originais) e encolhimento, que reduz o espaço de estados abstratos [Helmert et al., 2007]. O principal desafio dessas heurísticas é encontrar formas de encolher o espaço de estados abstrato sem perder informação. Hoffmann et al. [2014] propuseram adaptações das abstrações M\&s que só preservam informações de alcançabilidade da meta, explorando o fato de que essas informações de alcançabilidade não têm relação com o custo das ações.
\end{abstract}

Traps [Lipovetzky et al., 2016]. Uma trap é uma fórmula proposicional na forma normal disjuntiva que quando satisfeita em um estado $s$, também é satisfeita em todos os estados alcançáveis a partir de $s$ Lipovetzky et al. $[2016]^{3}$. Uma trap de beco-sem-saída é uma trap na forma norma disjuntiva em que todos os termos são mutuamente exclusivos com a meta. Para identificar uma $k$-trap, trap cujos termos têm tamanho máximo $k$, esse método analisa um grafo causal estendido em que os vértices têm conjunções com no máximo $k$ proposições. A trap de becos-sem-saída pode ser computada antes de inicializar a busca, considerando apenas os nós desse grafo que estiverem em exclusão mútua com a meta. Assim, durante a busca estados que satisfazem a trap são considerados becos-sem-saída. Assim como os métodos de consistência local, este método está limitado a um parâmetro $k$. Se forem necessárias mais de $k$ proposições para tornar um estado um beco-sem-saída, esse método não irá identificá-lo como beco-sem-saída.

\subsection{Discussão}

Nesse capítulo, apresentamos as principais heurísticas usadas na área de planejamento para estimar a distância (ou o custo) de um estado para alcançar um estado meta. Essas heurísticas são importantes em planejamento probabilístico para o cálculo de $V^{0}(s)$ e também podem detectar becos-sem-saída. No Capítulo 7, usaremos as heurísticas apresentadas nas seções 4.1 e 4.2 para avaliarmos o método proposto para a detecção de becos-sem-saída no Capítulo 6, o SWR-DE. As heurísticas da Seção 4.3 não serão avaliadas pois não estão disponíveis dentro do arcabouço de planejamento utilizado.

\footnotetext{
${ }^{3}$ Apesar do mesmo nome esse conceito é diferente do conceito trap definido por Kolobov et al. [2011] (Seção 3.2.2), que denotada um conjunto de estados.
} 


\section{Capítulo 5}

\section{Caracterização de GMDPs por verificação de modelos}

No Capítulo 3 fizemos uma análise de modelos que tratam problemas com becos-sem-saída. Neste capítulo, mostramos como os modelos SSP MDP, SSPADE MDP e SSPUDE MDP podem ser caracterizados. Para isso, utilizamos o formalismo da lógica temporal $\alpha$-CTL cuja semântica inclui ações.

\subsection{Planejamento não-determinístico}

Conforme vimos na Seção 2.4, em um problema de planejamento não-determinístico totalmente observável (Fully Observable Non-Deterministic Planning Task - FOND) (Definição 11), o agente conhece o estado atual do mundo, mas existe incerteza nos efeitos das ações. Diferente dos problemas de planejamento probabilístico a distribuição de probabilidade dos efeitos das ações não é conhecida. Assim, ao aplicar uma ação $a$ em um estado $s$, o agente pode ir para um conjunto de estados $S^{\prime} \subseteq S$, isto é, o agente sabe quais estados ele pode alcançar, mas não tem nenhuma informação adicional sobre qual dos estados deste conjunto será o próximo estado [Trevizan, 2006].

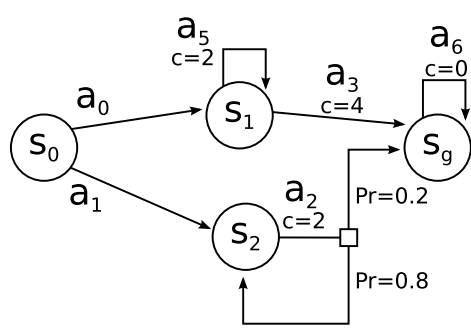

(a)

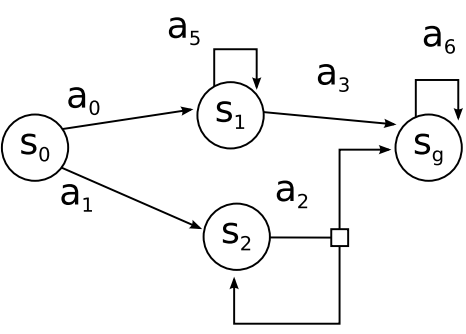

(b)

Figura 5.1: Diferença entre (a) um GMDP $\mathcal{M}$ e (b) um FOND $\mathcal{M}^{\text {nd }}$.

Podemos definir uma versão não-determinística de um GMDP $\mathcal{M}=\left\langle S, A, P, C, s_{0}, G\right\rangle$ como um FOND $\mathcal{M}^{n d}=\left\langle S, A, T, s_{0}, G\right\rangle$, em que as probabilidades são ignoradas, $T(s, a)=\left\{s^{\prime} \mid P\left(s^{\prime} \mid s, a\right)>0\right\}$, e as ações têm custo unitário (por isso, neste capítulo, omitimos a função custo). A Figura 5.1 mostra um GMDP $\mathcal{M}$ e o respectivo FOND $\mathcal{M}^{\text {nd }}$, no qual as probabilidades dos efeitos da ação $a_{2}$ são ignoradas.

Assim como em planejamento probabilístico, a solução para um FOND é dada por uma política $\pi: S \rightarrow A$. No entanto, como não existem probabilidades associadas aos efeitos das ações, os critérios para definir qual a melhor política em um FOND são definidos de forma qualitativa, a saber:

Política fraca: alcança um estado meta com uma chance não nula, isto é, pelo menos uma execução de uma política fraca termina em um estado meta. No entanto, devido à incerteza dos efeitos das ações, não há garantia que um estado meta seja alcançado; 
Política forte: com certeza alcança um estado meta sem visitar o mesmo estado duas vezes. Esse tipo de política leva o agente com um número finito de ações para a meta; e

Política forte-cíclica: em algum estágio chega a um estado meta. Uma política forte-cíclica pode conter ciclos, assim, sua execução pode gerar uma sequência infinita de estados.

Observamos que uma política fraca é otimista, pois assume que o melhor efeito de cada ação da política irá ocorrer. Políticas fortes são seguras, pois garantem que a meta será atingida em um número finito de passos [Pereira, 2007]. Para garantir que a meta será atingida em algum estágio, as políticas forte-cíclicas se baseiam na suposição de equidade [Cimatti et al., 2003], ou seja, supõem que se uma ação é executada em um estado por um número infinito de vezes, todos os efeitos dessa ação ocorrerão ao menos uma vez.

A Figura 5.2 ilustra os diferentes tipos de políticas. Nesse exemplo de FOND, existem apenas 4 políticas parciais possíveis: $\left.\pi_{1}=\left\{s_{0}: a_{1}, s_{1}: a_{4}, s_{4}: a_{8}\right\}, \pi_{2}=\left\{s_{0}: a_{2}, s_{2}: a_{5}\right\}, \pi_{3}=\left\{s_{0}: a_{3}, s_{3}: a_{6}\right\}\right)$ e $\left.\pi_{4}=\left\{s_{0}: a_{3}, s_{3}: a_{7}\right\}\right)$. Observe que a política $\pi_{1}$ sempre leva o agente para a meta em dois ou três passos, dependendo do efeito de $a_{4}$ que ocorrer, logo $\pi_{1}$ é uma política forte. A política $\pi_{2}$ com certeza leva o agente para a meta; note que a execução de $a_{5}$ no estado $s_{2}$ pode manter o agente no mesmo estado, o que configura um ciclo; logo, $\pi_{2}$ é uma política forte-cíclica. Observe que a execução de $\pi_{2}$ pode ser infinita, mas pela suposição de equidade assumimos que o efeito da ação $a_{5}$ que leva de $s_{2}$ para $s_{g}$ ocorrerá em algum momento, levando o agente para a meta. Finalmente, observamos que a política $\pi_{3}$ é uma política fraca pois pode alcançar $s_{g}$ ao executar a ação $a_{6}$ no estado $s_{3}$, porém pode ir para o estado $s_{5}$ e permanecer nele. A política $\pi_{4}$ não satisfaz nenhum dos critérios definidos anteriormente, pois nunca leva o agente para o estado meta $s_{g}$.

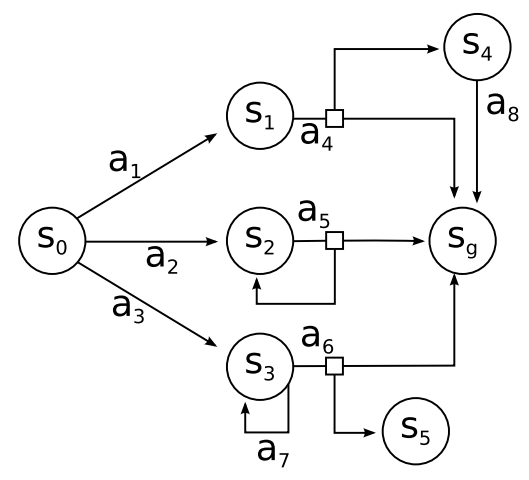

Figura 5.2: Exemplo de FOND.

Toda política forte é também uma política forte-cíclica e toda política forte-cíclica é também uma política fraca [Pereira, 2007].

Há diversas abordagens para resolver problemas de planejamento não-determinístico para gerar uma política forte, forte-cíclica ou fraca. Por exemplo, é possível utilizar algoritmos de busca ANDOR [Genesereth e Nourbakhsh, 1993]. Outra abordagem é utilizar a estratégia de replanejamento [Muise et al., 2012]. Nesse caso, um planejador clássico é utilizado para computar um plano em uma versão relaxada determinística do problema. Assim, o planejador executa o plano até alcançar um estado meta ou, caso encontre um estado inesperado, ele replaneja a partir do estado corrente. Existe ainda a abordagem baseada em métodos formais, chamada planejamento como verificação de modelos [Cimatti et al., 1997, 2003; Giunchiglia e Traverso, 1999; Pereira e Barros, 2008, 2012]. Nas próximas seções, apresentaremos essa abordagem com mais detalhes. Em seguida, mostramos como esse formalismo pode ser utilizado para caracterizar os modelos de planejamento probabilístico SSP MDP, SSPADE MDP e SSPUDE MDP. 


\subsection{Verificação de modelos}

Verificação é a tarefa de determinar se uma propriedade é satisfeita em um sistema. Uma abordagem formal para esse problema, baseada em lógicas temporais, é a verificação de modelos. Nessa abordagem o sistema é descrito por um modelo $\mathcal{K}$, e a propriedade que se deseja verificar é descrita por uma fórmula $\varphi$. Assim, um verificador de modelos deve dizer se o modelo $\mathcal{K}$ satisfaz a propriedade $\varphi$ a partir de um estado $s$ (denotado por $(\mathcal{K}, s) \vDash \varphi$ ) ou, caso não satisfaça, deve devolver um contraexemplo.

Em geral, o sistema $\mathcal{K}$ é descrito por uma estrutura de Kripke, um modelo formal utilizado para definir a dinâmica do ambiente ou do sistema (que assim como um GMDP pode ser representado por um grafo direcionado). A propriedade que se deseja verificar é dada por uma fórmula $\varphi$ descrita em uma lógica temporal, por exemplo, a lógica de tempo ramificado CTL (Computational Tree Logic) [Clarke e Emerson, 1982] descrita a seguir.

Definição 17 (Estrutura de Kripke). Seja $\mathbb{P}$ um conjunto não vazio finito de proposições atômicas. Uma estrutura de Kripke sobre $\mathbb{P}$ é um grafo de transição de estados $\mathcal{K}=\langle\mathcal{S}, \mathcal{L}, \mathcal{T}\rangle$, em que

- $\mathcal{S}$ é um conjunto de estados;

- $\mathcal{L}: \mathcal{S} \rightarrow 2^{\mathbb{P}}$ é uma função que rotula os estados; e

- $\mathcal{T}: \mathcal{S} \rightarrow 2^{\mathcal{S}}$ é uma função de transição de estados não-determinística.

$\triangle$

Como na Seção 2.4, $\mathbb{P}$ é um conjunto de proposições que descreve as propriedades do mundo, assim, $\mathcal{L}(s)$ denota quais propriedades são verdadeiras em um estado $s$.

Uma estrutura de Kripke $\mathcal{K}=\langle\mathcal{S}, \mathcal{L}, \mathcal{T}\rangle$ pode ser desdobrada em uma árvore de computação enraizada em um determinado estado $s \in \mathcal{S}$, denotada por $\Upsilon_{\mathcal{K}}^{s}$. Cada ramo dessa árvore é um caminho $\rho \in \Upsilon_{\mathcal{K}}^{s}$, uma sequência infinita de estados $\left\langle\rho_{0}, \rho_{1}, \rho_{2}, \cdots\right\rangle$, sendo $\rho_{i} \in \mathcal{S}, \forall i \geq 0, \rho_{0}=s$ e $\rho_{i} \in \mathcal{T}\left(\rho_{i-1}\right), \forall i \geq 1$. Assim, uma árvore de computação representa o conjunto de todos os possíveis caminhos de um modelo partindo de um determinado estado desse modelo.

\subsubsection{A lógica CTL}

Para formalizar as propriedades temporais podemos utilizar a lógica de tempo ramificado CTL (Computational Tree Logic) [Clarke e Emerson, 1982]. As fórmulas da lógica CTL são compostas por proposições atômicas, conectivos lógicos $(\neg, \wedge \mathrm{e} \vee$ ), quantificadores de caminho $(\exists$ e $\forall)$, e pelos operadores temporais: $\bigcirc$ (sucessor imediato), $\square$ (invariavelmente), $\diamond$ (finalmente) e $\sqcup$ (até que). Em CTL os operadores temporais devem ser precedidos imediatamente por um quantificador de caminhos $(\exists$ ou $\forall)$.

Definição 18. Seja $\mathbb{P}$ um conjunto de proposições atômicas. A sintaxe da lógica CTL é definida indutivamente como

$$
\varphi::=\top|\perp| p \in \mathbb{P}|\neg \varphi|\left(\varphi \wedge \varphi^{\prime}\right)\left|\left(\varphi \vee \varphi^{\prime}\right)\right| \exists \bigcirc \varphi|\forall \bigcirc \varphi| \exists \square \varphi|\forall \square \varphi| \exists\left(\varphi \sqcup \varphi^{\prime}\right) \mid \forall\left(\varphi \sqcup \varphi^{\prime}\right) . \triangle
$$

Os operadores temporais $\exists \diamond \varphi$ e $\forall \diamond \varphi$ são definidos da seguinte forma $\exists \diamond \varphi \doteq \exists(T \sqcup \varphi)$ e $\forall \diamond \varphi \doteq \forall(T \sqcup \varphi)$.

Uma fórmula CTL é interpretada sobre uma estrutura de Kripke e sua respectiva árvore de computação. A Figura 5.3 ilustra a semântica dos operadores temporais da lógica temporal CTL. Cada figura mostra a árvore de computação em uma estrutura de Kripke $\mathcal{K}=\langle\mathcal{S}, \mathcal{L}, \mathcal{T}\rangle$ a partir de um estado $s \in \mathcal{S}$.

Definição 19. Seja $\mathcal{K}=\langle\mathcal{S}, \mathcal{L}, \mathcal{T}\rangle$ uma estrutura de Kripke sobre $\mathbb{P}, s$ um estado em $\mathcal{S}$ e $\varphi$ uma fórmula CTL. Dizemos que o estado s no modelo $\mathcal{K}$ satisfaz $\varphi$, isto é $(\mathcal{K}, s) \vDash \varphi$, se:

- $(\mathcal{K}, s) \vDash T e(\mathcal{K}, s) \not \perp$; 
- $(\mathcal{K}, s) \vDash p$ sse $p \in \mathcal{L}(s)$;

- $(\mathcal{K}, s) \vDash \neg \varphi$ sse $s \not \varphi$;

- $(\mathcal{K}, s) \vDash \varphi \wedge \varphi^{\prime}$ sse $(\mathcal{K}, s) \vDash \varphi e(\mathcal{K}, s) \vDash \varphi^{\prime} ;$

- $(\mathcal{K}, s) \vDash \varphi \vee \varphi^{\prime}$ sse $(\mathcal{K}, s) \vDash \varphi$ ou $(\mathcal{K}, s) \vDash \varphi^{\prime}$;

- $(\mathcal{K}, s) \vDash \exists \bigcirc \varphi$ sse para algum caminho $\rho \in \Upsilon_{\mathcal{K}}^{s},\left(\mathcal{K}, \rho_{1}\right) \vDash \varphi$;

- $(\mathcal{K}, s) \vDash \forall \bigcirc \varphi$ sse para todo caminho $\rho \in \Upsilon_{\mathcal{K}}^{s},\left(\mathcal{K}, \rho_{1}\right) \vDash \varphi$;

- $(\mathcal{K}, s) \vDash \exists \square \varphi$ sse para algum caminho $\rho \in \Upsilon_{\mathcal{K}}^{s}$, e para todo $i \geq 0,\left(\mathcal{K}, \rho_{i} \vDash\right) \varphi$;

- $(\mathcal{K}, s) \vDash \forall \square \varphi$ sse para todo caminho $\rho \in \Upsilon_{\mathcal{K}}^{s}$, e para todo $i \geq 0,\left(\mathcal{K}, \rho_{i} \vDash\right) \varphi$;

- $(\mathcal{K}, s) \vDash \exists\left(\varphi \sqcup \varphi^{\prime}\right)$ sse para algum caminho $\rho \in \Upsilon_{\mathcal{K}}^{s}$, existe $j \geq 0$ tal que $\mathcal{K},\left(\rho_{j} \vDash \varphi^{\prime}\right)$, e para todo $i<$ $j,\left(\mathcal{K}, \rho_{i} \vDash \varphi\right) ; e$

- $(\mathcal{K}, s) \vDash \forall\left(\varphi \sqcup \varphi^{\prime}\right)$ sse para todo caminho $\rho \in \Upsilon_{\mathcal{K}}^{s}$, existe $j \geq 0$ tal que $\mathcal{K},\left(\rho_{j} \vDash \varphi^{\prime}\right)$, e para todo $i<$ $j,\left(\mathcal{K}, \rho_{i} \vDash \varphi\right)$.

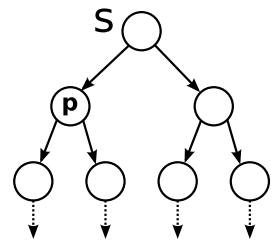

(a) $(\mathcal{K}, s) \vDash \exists \circ p$

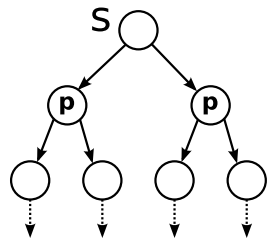

(e) $(\mathcal{K}, s) \vDash \forall \bigcirc p$

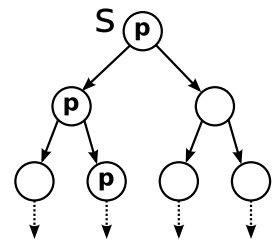

(b) $(\mathcal{K}, s) \vDash \exists \square p$

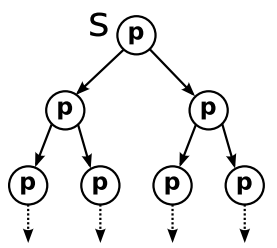

(f) $(\mathcal{K}, s) \vDash \forall \square p$

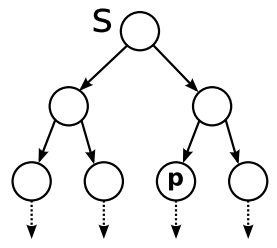

(c) $(\mathcal{K}, s) \vDash \exists \diamond p$

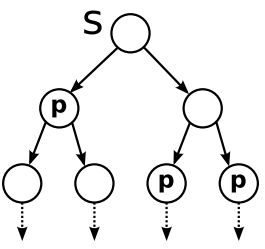

(g) $(\mathcal{K}, s) \vDash \forall \diamond p$

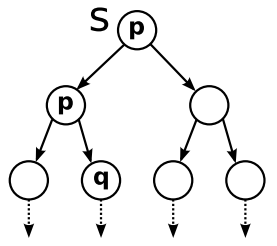

(d) $(\mathcal{K}, s) \vDash \exists(p \sqcup q)$

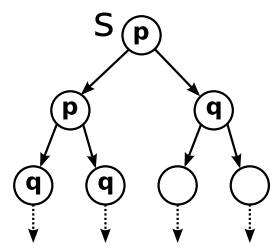

(h) $(\mathcal{K}, s) \vDash \forall(p \sqcup q)$

Figura 5.3: Semântica dos operadores temporais da lógica CTL [Pereira, 2007].

\subsubsection{Planejamento como verificação de modelos}

As técnicas de verificação de modelos podem ser adaptadas para resolver problemas de planejamento [Cimatti et al., 1997, 2003; Giunchiglia e Traverso, 1999]. Nesse caso, dado um FOND $\mathcal{M}=\left\langle S, A, T, s_{0}, G\right\rangle$, denotamos por $\mathcal{K}(\mathcal{M})=\langle\mathcal{S}, \mathcal{L}, \mathcal{T}\rangle$ a estrutura de Kripke relacionada ao espaço de transição de estados de $\mathcal{M}$ e $\psi$ uma fórmula lógica que representa o conjunto de estados meta $G$. Denotamos por $\varphi$ uma fórmula temporal que especifica a qualidade esperada de uma política que resolve $\mathcal{M}$ : fraca, forte-cíclica ou forte. Denotamos por $\mathcal{K}(\mathcal{M})_{\pi}$ o submodelo de $\mathcal{K}(\mathcal{M})$ que só inclui as transições da política $\pi$. Assim, se $\exists \pi:\left(\mathcal{K}(\mathcal{M})_{\pi}, s_{0}\right) \vDash \varphi$, o planejador devolve a política $\pi$; senão, ele devolve fracasso.

Podemos utilizar a lógica CTL para formalizar os três tipos de políticas para FOND. A propriedade de uma política fraca é denotada por $\varphi=\exists \diamond \psi$, que diz que existe um caminho que alcança um estado que satisfaz a meta. A propriedade de uma política forte é denotada por $\varphi=\forall \diamond \psi$, que diz que todos os caminhos alcançam um estado que satisfaz a meta. A propriedade de uma política forte-cíclica é denotada por $\varphi=\forall \square \exists \diamond \psi$, que diz que todos os caminhos alcançam um estado que satisfaz a meta a despeito de ciclos. Logo, dependendo da qualidade desejada, especificamos um problema diferente que satisfaça a propriedade a ser verificada. 
Em geral, técnicas de verificação de modelos são utilizadas para verificar se um modelo $\mathcal{M}$ satisfaz uma fórmula temporal $\varphi$. Assim, um verificador de modelos para CTL pode ser utilizado para validar a qualidade de uma determinada política $\pi$, checando se $\left(\mathcal{K}(\mathcal{M})_{\pi}, s_{0}\right) \vDash \varphi$. No entanto, como a semântica da lógica CTL não considera as ações, ela não é capaz de distinguir transições causadas por diferentes ações em um mesmo estado. Logo, ela não é adequada para sintetizar políticas como um resultado da verificação. Dessa forma, os algoritmos que usam verificação de modelos para planejamento baseados em CTL precisam de mecanismos extra-lógicos para sintetizar uma política [Pereira, 2007].

Na próxima seção apresentamos a lógica $\alpha$-CTL [Pereira e Barros, 2008, 2012], que inclui as ações em sua semântica, tornando-se mais adequada para lidarmos com problemas de planejamento. Esta lógica provê meios para sintetizar uma política sem a necessidade de mecanismos extra-lógicos. Em seguida, utilizamos essa lógica para caracterizar os modelos que lidam com becos-sem-saída de uma forma construtivista, bem como para a extração de um método de deteç̧ão de becos-sem-saída.

\subsection{3 $\alpha$-CTL: lógica de tempo ramificado para raciocínio sobre ações}

A lógica $\alpha$-CTL [Pereira e Barros, 2008, 2012] é uma lógica de tempo ramificado, cuja semântica é definida sobre estruturas de Kripke rotuladas, especialmente projetada para lidar com problemas de planejamento não-determinístico. Apesar da importância das ações na semântica da lógica $\alpha$-CTL, elas não são usadas para compor fórmulas $\alpha$-CTL. Isso se deve ao fato de que ao definir uma meta, impõe-se restrições apenas sobre os estados que poderão ser visitados durante a execução do plano e não sobre as ações que podem ser selecionadas. Lógicas existentes na literatura tais como De Nicola e Vaandrager [1990] e Pecheur e Raimondi [2006], que permitem restrições sobre ações, são inadequadas para nosso propósito.

\subsubsection{Sintaxe}

As fórmulas da lógica $\alpha$-CTL são compostas por proposições atômicas (por exemplo, T e $p$ ), conectivos lógicos $(\neg, \wedge \mathrm{e} \vee$ ), quantificadores de caminho $(\exists \mathrm{e} \forall)$, e os seguintes operadores temporais: $\odot($ sucessor imediato $), \odot($ invariantemente $), \odot($ finalmente $)$ e $\sqcup($ até que $)$.

Definição 20. Seja p uma proposição atômica. A sintaxe da lógica $\alpha$-CTL é definida indutivamente como

$$
\varphi::=\top|p| \neg p\left|\left(\varphi \wedge \varphi^{\prime}\right)\right|\left(\varphi \vee \varphi^{\prime}\right)|\exists \odot \varphi| \forall \odot \varphi|\exists \odot \varphi| \forall \odot \varphi\left|\exists\left(\varphi \sqcup \varphi^{\prime}\right)\right| \forall\left(\varphi \sqcup \varphi^{\prime}\right) .
$$

De acordo com a Definição 20, fórmulas bem formadas estão na forma normal negativa, assim, o escopo da negação é restrito a fórmulas atômicas. Isso facilita a definição da semântica de pontosfixos máximos e mínimos para os operadores temporais globais. Além disso, todos os operadores globais são prefixados por um quantificador de caminho $(\exists$ ou $\forall)$. Os operadores temporais derivados de $\odot$ são definidos como $\exists \odot \varphi \doteq \exists(T \sqcup \varphi)$ e $\forall \odot \varphi \doteq \forall(T \sqcup \varphi)$.

\subsubsection{Semântica}

Seja $\mathbb{P}$ um conjunto não vazio finito de proposições atômicas e $\mathbb{A}$ um conjunto não vazio de ações. Um modelo temporal da lógica $\alpha$-CTL sobre $(\mathbb{P}, \mathbb{A})$ é uma estrutura de Kripke rotulada $\mathcal{D}=\langle\mathcal{S}, \mathcal{L}, \mathcal{T}\rangle$, em que $\mathcal{S}$ é um conjunto de estados, $\mathcal{L}: \mathcal{S} \rightarrow 2^{\mathbb{P}}$ é uma função que rotula os estados, e $\mathcal{T}: \mathcal{S} \times \mathbb{A} \rightarrow 2^{\mathcal{S}}$ é uma função de transição de estados não-determinística. Além disso, estados terminais (i.e., estados nos quais a única ação aplicável é uma ação trivial $\tau$ ) em um modelo temporal persistem infinitamente no tempo (isto é, são estados absorventes).

Intuitivamente, a fórmula $\forall \odot \varphi$ é satisfeita em um estado $s \in \mathcal{S}$ se e somente se existe uma ação $\alpha$, cuja execução em $s$ necessariamente leva para um sucessor imediato $s^{\prime}$ de $s$ que satisfaz $\varphi$. A modalidade $\odot$ representa o conjunto de sucessores por $\alpha$-sucessores de $s$, para alguma ação particular $\alpha$ e o quantificador $\forall$ requer que todo estado nesse conjunto satisfaça $\varphi$. 


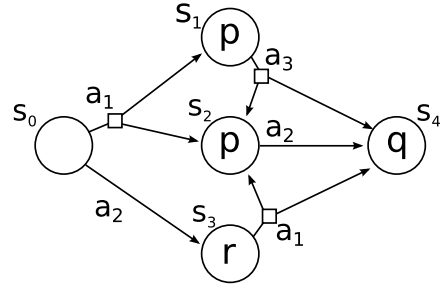

(a)

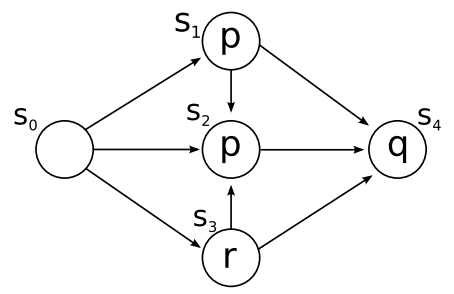

(b)

Figura 5.4: (a) Estrutura de Kripke rotulada $\mathcal{D}$. (b) Estrutura de Kripke $\mathcal{K}(\mathcal{D})$.

Para entender a diferença entre a semântica da lógica $\alpha$-CTL [Pereira e Barros, 2008] e a semântica da lógica CTL [Clarke e Emerson, 1982], considere a estrutura de Kripke rotulada $\mathcal{D}$ da Figura 5.4(a), e a estrutura de Kripke correspondente, sem ações, denotada por $\mathcal{K}(\mathcal{D})$ (Figura 5.4(b)). Claramente, de acordo com a semântica da lógica CTL, segue que $\left(\mathcal{K}(\mathcal{D}), s_{0}\right) \not \forall \circ p$ (porque $p$ não é satisfeito em $s_{3}$ ) e $\left(\mathcal{K}(\mathcal{D}), s_{0}\right) \not \forall \bigcirc \neg p$ (porque $\neg p$ não é satisfeito nos estados $s_{1}$ e $s_{2}$ ). Por outro lado, de acordo com a semântica da lógica $\alpha$-CTL, segue que $\left(\mathcal{D}, s_{0}\right) \vDash \forall \odot p$ (porque todos $a_{1}$-sucessores de $s_{0}$ satisfazem $p$ ) e $\left(\mathcal{D}, s_{0}\right) \vDash \forall \odot \neg p$ (porque todos $a_{2}$-sucessores de $s_{0}$ satisfazem $\neg p)$. De fato, cada ocorrência da modalidade $\odot$ pode instanciar uma ação $\alpha \in \mathbb{A}$ diferente e, consequentemente, a quantificação pode ser feita sobre diferentes conjuntos de $\alpha$-sucessores do estado $s_{0}$. No entanto, vale a pena observar que $\left(\mathcal{D}, s_{0}\right) \vDash \forall \odot p \wedge \forall \odot \neg p$ não significa que existe uma política que alcance, simultaneamente, ambas as submetas $p$ e $\neg p$ (uma meta impossível que seria especificada como $\forall \odot(p \wedge \neg p))$. Essa possibilidade é muito importante em planejamento, uma vez que se o agente não pode escolher suas ações (para atingir suas metas), ele não pode planejar.

A definição formal da semântica da lógica $\alpha$-CTL é baseada no conceito de pré-imagem. Intuitivamente, a pré-imagem forte (fraca) de um conjunto de estados $Y$ é o conjunto de estados $X$ a partir dos quais pode-se necessariamente (eventualmente) alcançar um estado em $Y$.

Definição 21 (Pré-imagem fraca e pré-imagem forte). Seja $Y \subseteq \mathcal{S}$ um conjunto de estados. A pré-imagem fraca de $Y$, denotada por $\mathcal{T}_{\exists}^{-}(Y)$, é o conjunto $\{s \in \mathcal{S}: \exists a \in \mathbb{A} .(\mathcal{T}(s, a) \cap Y) \neq \varnothing\}$, e a pré-imagem forte de $Y$, denotada por $\mathcal{T}_{\forall}^{-}(Y)$, é o conjunto $\{s \in \mathcal{S}: \exists a \in \mathbb{A} . \varnothing \neq \mathcal{T}(s, a) \subseteq Y\} . \quad \triangle$

Por exemplo, considerando o modelo temporal $\mathcal{D}$ (Figura 5.4(a)), a pré-imagem forte do conjunto de estados $Y=\left\{s_{4}\right\}$ é $X=\left\{s_{2}\right\}$, uma vez que $s_{2}$ é o único estado em $\mathcal{D}$ a partir do qual o estado $s_{4}$ pode ser atingido após executar uma única ação. Enquanto isso, a pré-imagem fraca do conjunto de estados $Y=\left\{s_{4}\right\}$ é $X=\left\{s_{1}, s_{2}, s_{3}\right\}$, uma vez que os três estados possuem alguma ação aplicável que pode levar para o estado $s_{4}$.

A semântica dos operadores temporais locais $(\exists \odot$ e $\forall \odot)$ é dada pelas funções de pré-imagem, enquanto, a semântica dos operadores temporais globais $(\exists \odot, \forall \odot, \exists \bullet$ e $\forall \bullet)$ é derivada da semântica dos operadores temporais locais, usando operações de ponto fixo mínimo $(\mu)$ e máximo $(\nu)$.

Definição 22. Seja $\mathcal{D}=\langle\mathcal{S}, \mathcal{L}, \mathcal{T}\rangle$ um modelo temporal com assinatura $(\mathbb{P}, \mathbb{A})$. A intensão de uma fórmula em $\alpha$-CTL $\varphi$ em $\mathcal{D}$ (ou um conjunto de estados que satisfaz $\varphi$ em $\mathcal{D}$ ), denotada por $\llbracket \varphi \rrbracket_{\mathcal{D}}$, é definida como:

- $\llbracket p \rrbracket_{\mathcal{D}}=\{s: p \in \mathcal{L}(s)\} ; \llbracket \top \rrbracket_{\mathcal{D}}=\mathcal{S} ; \llbracket \perp \rrbracket_{\mathcal{D}}=\varnothing ;$

- $\llbracket \neg p \rrbracket_{\mathcal{D}}=\mathcal{S} \backslash \llbracket p \rrbracket_{\mathcal{D}}$

- $\llbracket\left(\varphi \wedge \varphi^{\prime}\right) \rrbracket_{\mathcal{D}}=\llbracket \varphi \rrbracket_{\mathcal{D}} \cap \llbracket \varphi^{\prime} \rrbracket_{\mathcal{D}} ;$

- $\llbracket\left(\varphi \vee \varphi^{\prime}\right) \rrbracket_{\mathcal{D}}=\llbracket \varphi \rrbracket_{\mathcal{D}} \cup \llbracket \varphi^{\prime} \rrbracket_{\mathcal{D}}$

- $\llbracket \exists \odot \varphi \rrbracket_{\mathcal{D}}=\mathcal{T}_{\exists}^{-}\left(\llbracket \varphi \rrbracket_{\mathcal{D}}\right)$;

- $\llbracket \forall \odot \varphi \rrbracket_{\mathcal{D}}=\mathcal{T}_{\forall}^{-}\left(\llbracket \varphi \rrbracket_{\mathcal{D}}\right)$; 
- $\llbracket \exists \oslash \varphi \rrbracket_{\mathcal{D}}=\nu Y .\left(\llbracket \varphi \rrbracket_{\mathcal{D}} \cap \mathcal{T}_{\exists}^{-}(Y)\right)$;

- $\llbracket \forall \bowtie \varphi \rrbracket_{\mathcal{D}}=\nu Y .\left(\llbracket \varphi \rrbracket_{\mathcal{D}} \cap \mathcal{T}_{\forall}^{-}(Y)\right)$;

- $\llbracket \exists\left(\varphi \cdot \varphi^{\prime}\right) \rrbracket_{\mathcal{D}}=\mu Y .\left(\llbracket \varphi^{\prime} \rrbracket_{\mathcal{D}} \cup\left(\llbracket \varphi \rrbracket_{\mathcal{D}} \cap \mathcal{T}_{\exists}^{-}(Y)\right)\right) ; e$

- $\llbracket \forall\left(\varphi \sqcup \varphi^{\prime}\right) \rrbracket_{\mathcal{D}}=\mu Y .\left(\llbracket \varphi^{\prime} \rrbracket_{\mathcal{D}} \cup\left(\llbracket \varphi \rrbracket_{\mathcal{D}} \cap \mathcal{T}_{\forall}^{-}(Y)\right)\right)$.

Definição 23. Seja s um estado em um modelo temporal $\mathcal{D}$, e $\varphi$ uma fórmula $\alpha$-CTL. A relação de satisfazibilidade da lógica $\alpha$-CTL é definida como: $(\mathcal{D}, s) \vDash \varphi \Leftrightarrow s \in \llbracket \varphi \rrbracket_{\mathcal{D}}$.

A Figura 5.5 ilustra a semântica da lógica $\alpha$-CTL. Note que os ramos de cada árvore de computação são rotulados por ações. Observamos que os quantificadores existencias $(\exists \odot, \exists \odot, \exists \odot$ e $\exists \bullet$ ) são muito parecidos com o da lógica CTL, sendo necessário apenas que algum caminho da árvore satisfaça a fórmula $\varphi$. Enquanto isso, os quantificadores universais $(\forall \odot, \forall \odot, \forall \odot$ e $\forall \bullet)$ são diferentes dos quantificadores universais da lógica CTL, pois é necessário que todos os caminhos por uma ação satisfaçam a fórmula $\varphi$.

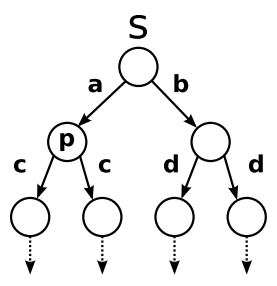

(a) $(\mathcal{D}, s) \vDash \exists \odot p$

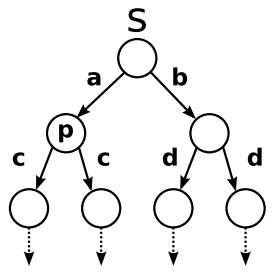

(e) $(\mathcal{D}, s) \vDash \forall \odot p$

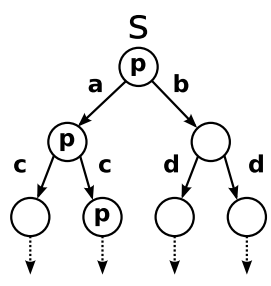

(b) $(\mathcal{D}, s) \vDash \exists \boxminus p$

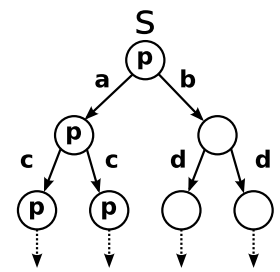

(f) $(\mathcal{D}, s) \vDash \forall \boxminus p$

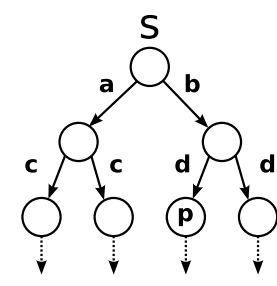

(c) $(\mathcal{D}, s) \vDash \exists \diamond p$

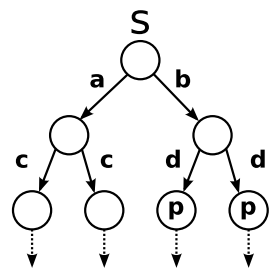

(g) $(\mathcal{D}, s) \vDash \forall \odot p$

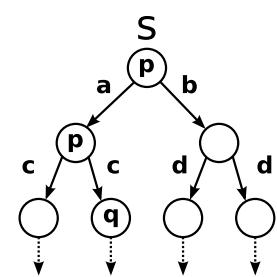

(d) $(\mathcal{D}, s) \vDash \exists(p \sqcup q)$

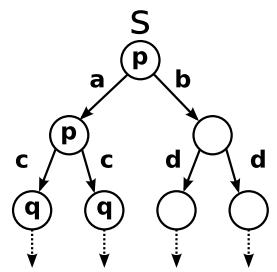

(h) $(\mathcal{D}, s) \vDash \forall(p \sqcup q)$

Figura 5.5: Semântica dos operadores temporais da lógica $\alpha$-CTL [Menezes, 2014].

\subsection{GMDPs com becos-sem-saída: uma formalização por verificação de modelos}

Alguns becos-sem-saída são fáceis de serem reconhecidos (por exemplo, estados absorventes que não satisfazem a meta), chamados becos-sem-saída explícitos. Outros podem ser difíceis de detectar, chamados becos-sem-saída implícitos (por exemplo, becos-sem-saída pertencentes a uma trap permanente que não contém um estado meta). Neste trabalho, estamos interessados na detecção de becos-sem-saída implícitos.

\subsubsection{Caracterizando o conjunto de becos-sem-saída de um GMDP}

Seja $\mathcal{M}=\left\langle S, A, P, C, G, s_{0}\right\rangle$ um GMDP e $\mathcal{M}^{\text {nd }}$ o FOnD correspondente. Seja $\mathcal{D}\left(\mathcal{M}^{n d}\right)$ a estrutura de Kripke rotulada de $\mathcal{M}^{\text {nd }}$, que abreviaremos por $\mathcal{D}(\mathcal{M})$. Seja $\varphi$ uma fórmula proposicional que caracteriza o conjunto de estados meta $G$. A fórmula $\alpha$-CTL $\exists \odot \varphi$ (existe uma trajetória que finalmente alcança um estado meta) pode ser usada para expressar o conjunto $\mathcal{R}$ de estados a partir dos quais um estado meta pode ser alcançado. Como $\exists \odot \varphi$ é equivalente a $\exists(\top \sqcup \varphi)$, de acordo com a Definição 22 , o conjunto de estados $\mathcal{R}$ é a intensão da fórmula $\alpha$-CTL:

$$
\left.\mathcal{R}=\llbracket \exists \odot \varphi \rrbracket_{\mathcal{D}(\mathcal{M})}=\mu Y .\left(\llbracket \varphi \rrbracket_{\mathcal{D}(\mathcal{M})} \cup \mathcal{T}_{\exists}^{-}(Y)\right)\right) .
$$


Intuitivamente, o conjunto $\mathcal{R}$ de estados a partir dos quais um estado meta é possivelmente alcançado é o ponto fixo mínimo da função de pré-imagem fraca começando com $G ; \llbracket \varphi \rrbracket_{\mathcal{D}(\mathcal{M})}$ é o conjunto de estados meta $G$ e $\mathcal{T}_{\exists}^{-}(Y)$ é a pré-imagem fraca de um conjunto de estados $Y$. Assim, o conjunto de estados $\mathcal{R}$ a partir dos quais um estado meta pode ser atingido é computado iterativamente pela atribuição $Y^{i+1}:=\llbracket \varphi \rrbracket_{\mathcal{D}(\mathcal{M})} \cup \mathcal{T}_{\exists}^{-}\left(Y^{i}\right)$, com $Y^{0}=\varnothing$, até atingir o ponto fixo.

Teorema 1. O conjunto de estados $\mathcal{R}$, computado pela Equação 5.1, é o conjunto de todos os estados a partir dos quais existe uma trajetória que finalmente alcança um estado meta.

Demonstração. Depois da primeira iteração, $Y^{1}=G$ e, claramente, $Y^{1}$ contém todos os estados a partir dos quais existe uma trajetória (com tamanho 0) para um estado meta. Supondo que na $i$-ésima iteração $Y^{i}$ é o conjunto dos estados a partir dos quais existe uma trajetória para a meta com no máximo $i-1$ passos. Então, a pré-imagem fraca de $Y^{i}$, i.e. $\mathcal{T}_{\exists}^{-}\left(Y^{i}\right)=\{s \in \mathcal{S}: \exists a \in$ $\left.\mathbb{A} .\left(\mathcal{T}(s, a) \cup Y^{i}\right) \neq \varnothing\right\}$, é o conjunto de todos os estados que podem alcançar $Y^{i}$ com no máximo um passo. Assim, todos os estados em $Y^{i+1}:=G \cup \mathcal{T}_{\exists}^{-}\left(Y^{i}\right)$ podem alcançar um estado meta com $i$ passos. Como $S$ é um conjunto finito, com certeza existe um ponto fixo e, claramente, esse ponto fixo é o conjunto $\mathcal{R}$.

Corolário 1. Seja $S$ o conjunto de estados de um GMDP $\mathcal{M}$, e $\mathcal{R} \subseteq S$ o conjunto de estados a partir dos quais existe ao menos uma trajetória para um estado meta em $\mathcal{M}$. Então, o conjunto de becos-sem-saída em $\mathcal{M}$, denotado por $\mathcal{D} \mathcal{E}$, é $S \backslash \mathcal{R}$.

Em CTL uma política forte-cíclica é definida pela fórmula $\phi=\forall \square \exists \diamond \psi$. Conforme Pereira [2007] e Menezes [2014] uma política forte-cíclica seria então definida de forma análoga pela fórmula $\alpha$-CTL $\phi=\forall \bullet \exists \diamond \psi$, assim, o conjunto de estados que em algum estágio alcançam um estado meta seria a intensão dessa fórmula, $\mathcal{R}^{*}=\llbracket \forall \boxminus \exists \odot \psi \rrbracket$. Porém, essa caracterização está correta somente em problemas que não incluem certos tipos de ciclos. Para uma caracterização completa do conjunto $\mathcal{R}^{*}$ é preciso estender a semântica de $\alpha$-CTL conforme discutiremos no final deste capítulo.

\subsubsection{SSPADE E SSPUDES}

Seja $\mathcal{M}=\left\langle S, A, P, C, G, s_{0}\right\rangle$ um GMDP e $\mathcal{D}(\mathcal{M})$ o respectivo grafo de transição de estados rotulado sem probabilidades. Seja $\varphi$ uma fórmula proposicional denotando o conjunto de estados meta $G$. Seja $\mathcal{R}$ o conjunto de estados a partir dos quais é possível alcançar um estado meta, e $\mathcal{D E}=S \backslash \mathcal{R}$ o conjunto de todos os becos-sem-saída de $\mathcal{M}$. Seja $\mathcal{R}^{*} \subseteq \mathcal{R}$ o conjunto de todos os estados que necessariamente alcançam um estado meta ${ }^{1}$, isto é, estados com $P^{G}=1$. Assumindo que $C$ seja uma função de custo positiva $\left(C: S \rightarrow \mathbb{R}^{+}\right)$, seguem as seguintes proposições.

Proposição 7. $O$ GMDP $\mathcal{M}$ é um SSPADE MDP, se e somente se, $s_{0} \in \mathcal{R}^{*}$.

Rascunho da prova. (i) Como $\mathcal{M}$ possui uma política própria a partir do estado inicial (suposição A1), o modelo $\mathcal{D}(\mathcal{M})$ possui ao menos uma política em que todas as trajetórias enraizadas em $s_{0}$ em algum momento alcançam um estado meta, $\log s_{0} \in \mathcal{R}^{*}$. (ii) Como $\mathcal{R}^{*}$ é o conjunto de todos os estados a partir dos quais necessariamente é possível alcançar um estado meta, se $s_{0} \in \mathcal{R}^{*}$, então existe uma política tal que todas as trajetórias iniciadas em $s_{0}$ necessariamente alcançam um estado meta, i.e, existe uma política própria a partir de $s_{0}$ (suposição A1). Como assumimos que $C$ é uma função positiva, temos que toda política imprópria a partir de $s_{0}$ tem um custo esperado infinito (suposição A2), logo i.e, $\mathcal{M}$ é um SSPADE MDP.

Proposição 8. $O$ GMDP $\mathcal{M}$ é um $\mathrm{SSPUDE}$ MDP, se e somente se, $s_{0} \in \mathcal{R}$.

Rascunho da prova. (i) Como $\mathcal{M}$ é um Sspude mDP, então $\mathcal{M}$ possui ao menos uma política com probabilidade maior que zero de alcançar um estado meta a partir do estado inicial, logo, existe ao menos uma trajetória em $\mathcal{D}(\mathcal{M})$ que leva o agente de $s_{0}$ para um estado que satisfaz $\varphi$, dessa

\footnotetext{
${ }^{1} \mathrm{O}$ apêndice $\mathrm{C}$ mostra como este conjunto pode ser calculado.
} 
forma, pelo Teorema 1 temos que $s_{0} \in \mathcal{R}$. (ii) Uma vez que $\mathcal{R}$ é o conjunto de estados a partir dos quais tanto um estado meta quanto um beco-sem-saída podem ser alcançados, se $s_{0} \in \mathcal{R}$, então existe uma política com probabilidade maior que zero de levar o agente do estado inicial para o estado meta, $\log$ $\mathcal{M}$ tem solução e trata-se de um SSPUDE MDP.

Proposição 9. Se $\mathcal{D} \mathcal{E}=\varnothing$ então $\mathcal{R}=\mathcal{R}^{*}$.

Rascunho da prova. Suponha que $\mathcal{D} \mathcal{E}=\varnothing$ e $\mathcal{R} \neq \mathcal{R}^{*}$. Uma vez que $\mathcal{R}^{*} \subseteq \mathcal{R}$, segue que $\mathcal{R} \backslash \mathcal{R}^{*} \neq \varnothing$. Dessa forma, deve existir um estado $s \in \mathcal{R} \backslash \mathcal{R}^{*}$ que pode alcançar um beco-sem-saída. Mas isso é uma contradição, uma vez que $\mathcal{D E}=\varnothing$.

Proposição 10. $O$ GMDP $\mathcal{M}$ é um $\mathrm{SSP}$ MDP, se e somente se, $\mathcal{D} \mathcal{E}=\varnothing$.

Rascunho da prova. (i) Se $\mathcal{M}$ é um SSP MDP então, existe uma política própria (suposição S1), isto é, todo estado possui uma política com probabilidade um de levar o agente para um estado meta. Logo, é possível alcançar um estado que satisfaz $\varphi$ a partir de todos os estados em $\mathcal{D}(\mathcal{M})$, portanto $\mathcal{D E}=\varnothing$. (ii) Se $\mathcal{D E}=\varnothing$, como $C(s)>0$, existe ao menos uma política própria e toda política imprópria tem um custo acumulado infinito nos estados com $P^{G}<1$. Portanto, $\mathcal{M}$ é um SSP MDP.

A Figura 5.6 mostra as relações entre os conjuntos $G$ (estados meta), $\mathcal{R}^{*}$ (estados que necessariamente levam para um estado meta), e $\mathcal{R}$ (estados que possivelmente levam a um estado meta). Como pudemos ver, baseados nesses conjuntos e em qual deles se encontra o estado inicial, os diferentes tipos de GMDPs podem ser precisamente caracterizados.

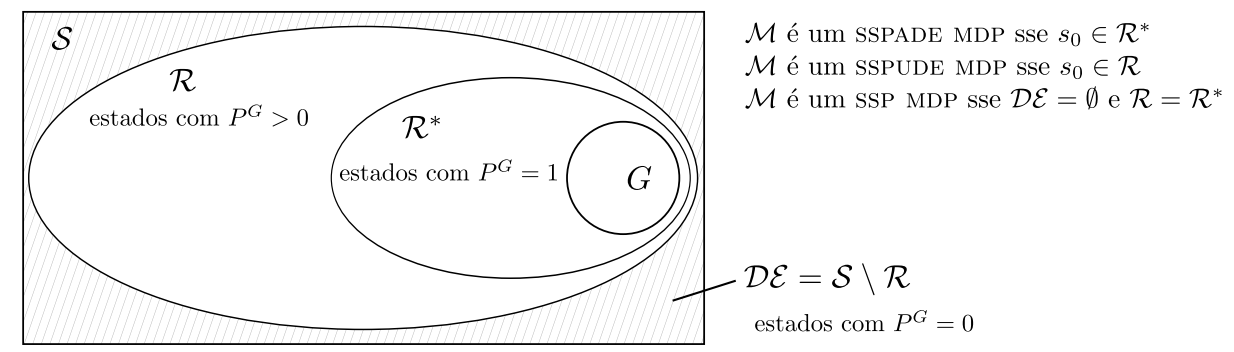

Figura 5.6: Relação entre os tipos de GMDPs. A região destacada indica o conjunto de becossem-saída $\mathcal{D E}$.

\subsection{Algoritmo enumerativo}

O planejador baseado em $\alpha$ CTL (PACTL) [Pereira, 2007] é capaz de sintetizar uma política cujo submodelo satisfaz propriedades descritas em $\alpha$-CTL, incluindo metas estendidas que dizem que uma propriedade $\varphi_{1}$ deve ser satisfeita até alcançar um estado em que outra propriedade $\varphi_{2}$ seja satisfeita. No entanto, para o propósito deste trabalho estamos interessados apenas em um fragmento da lógica $\alpha$-CTL. Nesta seção, apresentamos os métodos do algoritmo PACTL necessários para computar $\mathcal{R}$.

O Algoritmo 5.1 mostra como $\mathcal{R}$ pode ser computado utilizando apenas operações entre conjuntos de estados. Observe que esse algoritmo segue estritamente as definições apresentadas na Seção 5.2 .3 .

O Algoritmo 5.1 recebe como entrada o conjunto de proposições que descreve a meta $\mathbb{G} \subseteq \mathbb{P}$. Para entender esse algoritmo fazemos um paralelo com as operações da lógica $\alpha$-CTL. Como $\varphi$ é uma conjunção das proposições de $\mathbb{G}$, os conjunto de estados meta $G=\llbracket \varphi \rrbracket_{\mathcal{D}(\mathcal{M})}$ é o conjunto dos estados em que todas as proposições de $\mathbb{G}$ são verdadeiras (Linha 2). A função WeAkPreImage computa a função de pré-imagem $\mathcal{T}_{\exists}^{-}(Y)$ (Definição 21). Finalmente, o algoritmo principal computa o ponto fixo da pré-imagem fraca do conjunto de estados meta $G$. Nesse algoritmo, $X$ armazena o conjunto de estados $Y^{i-1}$ e $Y$ o conjunto de estados $Y^{i}$. 


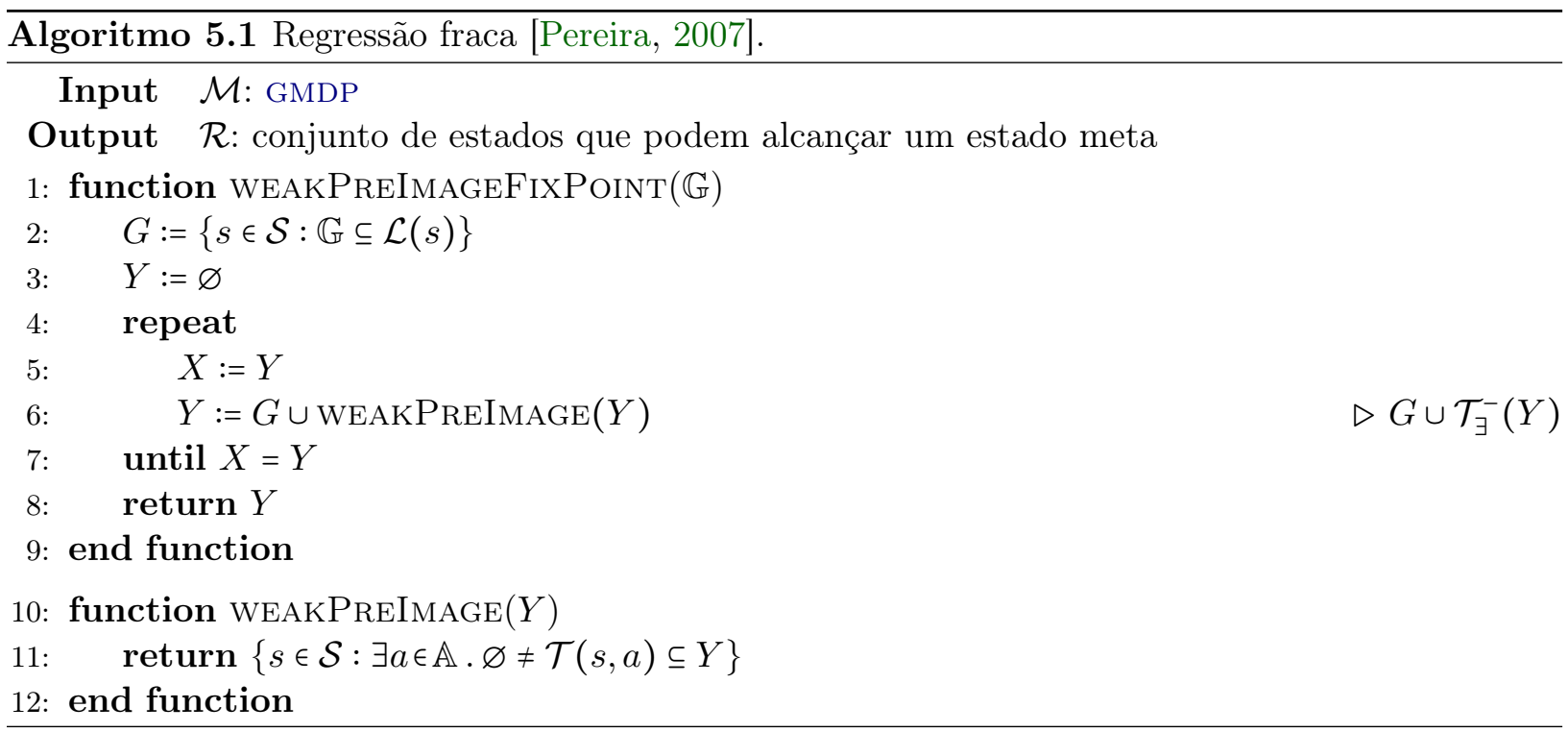

A Figura 5.7 mostra um exemplo da execução do algoritmo WeAKPREImageFixPoint. Nessa figura, destacamos o conjunto de estados $Y$ a cada iteração do laço principal do algoritmo. Inicialmente $Y^{0}=\varnothing$, após a primeira iteração tempos $Y^{1}=G \cup \mathcal{T}_{\exists}^{-}\left(Y^{0}\right)=G \cup \varnothing=\left\{s_{g}\right\}$. Em seguida, $Y^{2}=G \cup \mathcal{T}_{\exists}^{-}\left(Y^{1}\right)=\left\{s_{g}\right\} \cup\left\{s_{1}, s_{2}, s_{3}, s_{4}\right\}=\left\{s_{g}, s_{1}, s_{2}, s_{3}, s_{4}\right\}=$. Finalmente, $\mathcal{R}=Y^{3}=Y^{4}=$ $\left\{s_{g}, s_{1}, s_{2}, s_{3}, s_{4}, s_{0}\right\}$. Observamos assim, que o único estado nesse exemplo que não faz parte de $\mathcal{R}$ é o beco-sem-saída $s_{5}$.

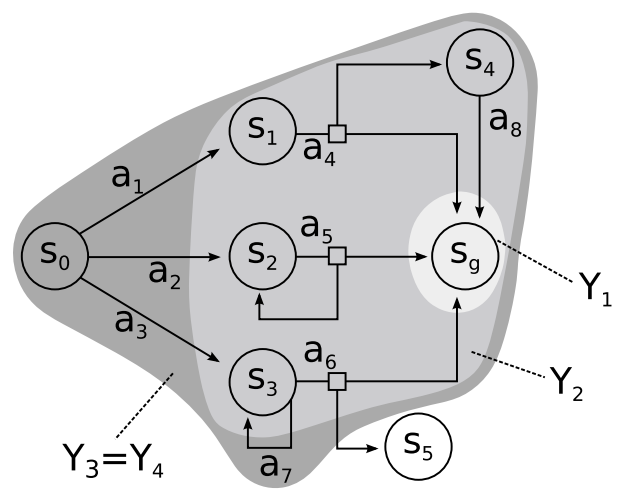

Figura 5.7: Funcionamento do algoritmo weAkPreImageFixpoint. $Y^{i}$ é o conjunto $Y$ do ponto fixo mínimo na i-ésima iteração.

\subsection{Cálculo de $\mathcal{R}^{*}$}

Como mencionamos anteriormente, em $\alpha$-CTL uma política forte-cíclica seria definida pela fórmula $\phi=\forall \boxminus \exists \diamond \psi$. No entanto, essa fórmula pode ser satisfeita em um estado $s \in S$ que nem sempre alcança um estado meta, caso $s$ pertença a um ciclo e algum estado desse ciclo possa alcançar um estado meta. A Figura 5.8(a) mostra um GMDP em que isso ocorre, no estado $s_{3}$, a ação $a_{7}$ sempre leva para um estado a partir do qual é possível alcançar o estado meta $s_{g}$. A seguir, mostramos como é computado o submodelo que satisfaz $\forall \bullet \exists \diamond \psi$. Ao final, propomos uma extensão da lógica $\alpha$-CTL para contornar esse problema.

Note que a computação da pré-imagem (Definição 21) devolve um conjunto de estados, no entanto, para sintetizar uma política forte-cíclica, é necessário utilizar uma representação para submodelos, isto é, uma estrutura que represente tanto estados como transições [Pereira, 2007, p. 75]. A Figura 5.8, mostra como o submodelo de um FOND $\mathcal{M}$ (Figura 5.8(a)) que satisfaz $\forall \bullet \exists \diamond \psi$ 
é calculado, sendo que $s_{g}$ satisfaz a meta $\psi$.

A Figura 5.8(b) mostra o submodelo que satisfaz a fórmula $\exists \odot \psi$, denotamos esse submodelo por $X$. A computação desse submodelo é muito parecida com a computação do conjunto $\mathcal{R}$. Observe que o conjunto de estados de $X$ coincide com o conjunto de estados que pode alcançar um estado meta $\mathcal{R}$. Assim, os becos-sem-saída $s_{4}$ e $s_{5}$ não fazem parte do submodelo $X$.

O submodelo que satisfaz $\forall \bullet \exists \odot \psi$ é computado através de um ponto fixo máximo, sendo, $Y$ inicializado com todo o submodelo $X$, isto $Y^{0}=X$ (Figura 5.8(a)). A seguir, o algoritmo calcula a pré-imagem forte de $\mathcal{T}_{\forall}^{-}\left(Y^{0}\right)$ e faz a intersecção com o submodelo $Y^{0}$ que pode alcançar um estado meta, isto é $Y^{1}=Y^{0} \cap \mathcal{T}_{\forall}^{-}\left(Y^{0}\right)=X \cap \mathcal{T}_{\forall}^{-}\left(Y^{0}\right)$ (Figura 5.8(c)). Essa operação é repetida, obtendo o submodelo $Y^{2}=X \cap \mathcal{T}_{\forall}^{-}\left(Y^{1}\right)$ (Figura 5.8(d)). Essa operação remove transições de $Y^{1}$ que podem levar para algum estado que não pertence a $Y^{1}$, eliminando as transições $\left(s_{1}: a_{4}\right)$ e $\left(s_{3}: a_{6}\right)$, que podiam levar para os estados $s_{4}$ e $s_{5}$. Com a próxima pré-imagem forte, $Y^{3}=X \cap \mathcal{T}_{\forall}^{-}\left(Y^{2}\right)$ (Figura 5.8(d)), a transição $\left(s_{0}: a_{1}\right)$ é eliminada. Finalmente, o ponto fixo é alcançado, pois $Y^{4}=$ $X \cap \mathcal{T}_{\forall}^{-}\left(Y^{3}\right)=Y^{3}$.

Observamos que a pré-imagem forte de $Y^{1}$ mantém o estado $s_{3}$, devido à presença do ciclo criado pela ação $a_{7}$ no estado $s_{3}$. Por isso esse estado não é removido do submodelo que satisfaz a fórmula $\forall \bullet \exists \odot \psi$. Observamos assim, que pela semântica da lógica $\alpha$-CTL, podemos acabar mantendo estados que pertencem a um ciclo e não satisfazem a qualidade esperada por um política forte-cíclica.

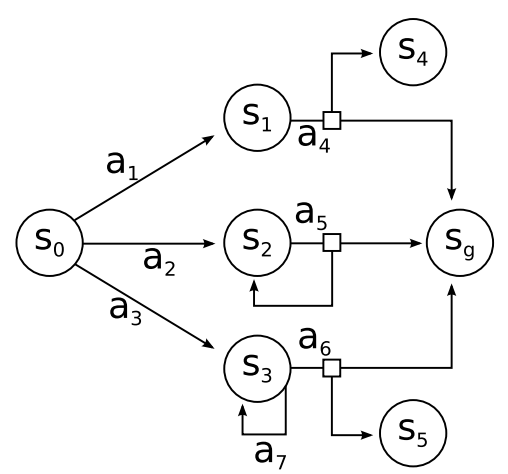

(a)

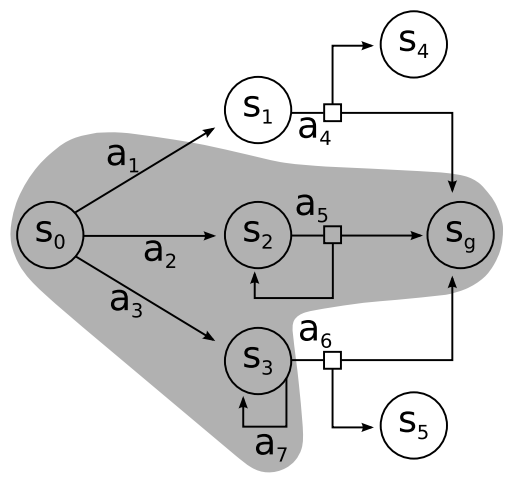

(c)

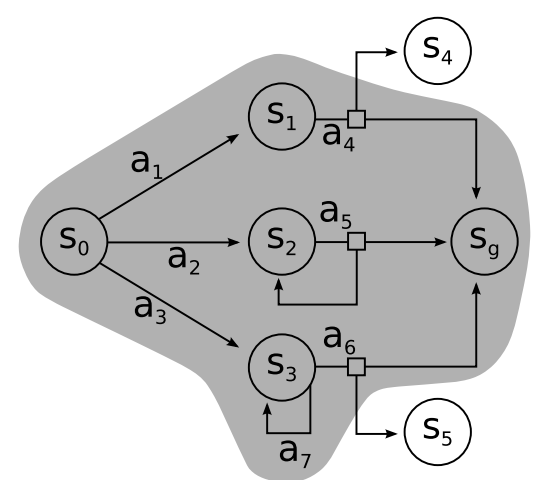

(b)

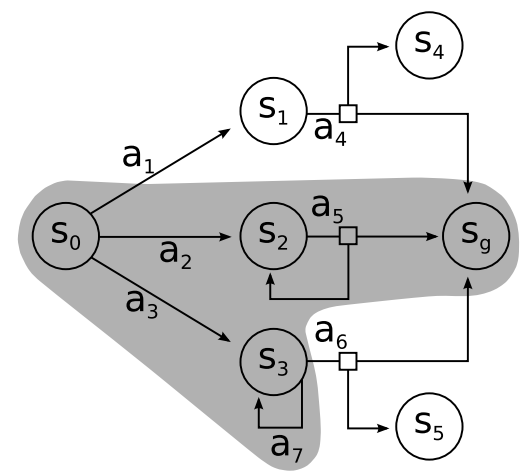

(d)

Figura 5.8: Computação do ponto fixo da fórmula $\phi=\forall \boxminus \exists \diamond \psi$. A área em destaque mostra o conjunto $Y$ a cada iteração: 5.8(a) $Y^{0}$, 5.8(b) $Y^{1}, 5.8(c) Y^{2}$ e 5.8(d) $Y^{3}=Y^{4}$.

Para eliminar estados que fazem parte de um ciclo, é necessário calcular novamente o conjunto $X=\exists \diamond \psi$ considerando apenas o submodelo $Y^{3}$. Na Figura 5.9, mostramos como seria o novo submodelo $X$ caso apenas o submodelo calculado anteriormente, $Y^{3}$, fosse considerado como domínio. Observe que, nesse caso, a regressão fraca não incluiria o estado $s_{3}$ pois a ação $a_{6}$ não faz parte de $Y^{3}$. 


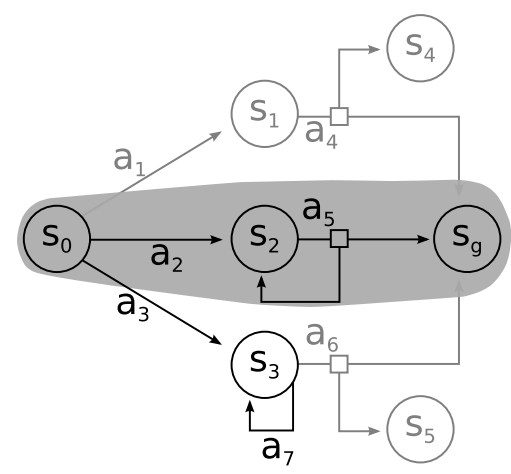

Figura 5.9: Resultado esperado da regressão forte-cíclica. A área em destaque mostra o resultado da regressão fraca. Transições e estados em cinza claro são desconsideradas pela operação de regressão fraca.

Percebemos que para computar uma política forte-cíclica é necessário alterar o domínio em que o ponto fixo mínimo da fórmula $\exists \diamond \psi$ é calculado. No entanto, a lógica $\alpha$-CTL não possui nenhum operador que permite alterar o domínio em que o ponto fixo é computado. A equação a seguir mostra como o conjunto $\mathcal{R}^{*}$ poderia ser computado utilizando duas operações de ponto-fixo.

$$
\mathcal{R}^{*}=\nu Y \cdot\left(\mu X .\left(\llbracket \psi \rrbracket_{\mathcal{D}(\mathcal{M})} \cup \mathcal{T}_{\exists}^{-}(X)\right)_{Y} \cap \mathcal{T}_{\forall}^{-}(Y)\right)_{\mathcal{D}(\mathcal{M})} .
$$

Observe que nesse caso, o submodelo que satisfaz $\exists \odot \psi$ é computado utilizando o submodelo $Y$ como domínio.

Ainda precisamos mostrar que a nova função é monótona, para mostrar que ela tem um ponto fixo. Observamos ainda que o submodelo calculado por esse novo ponto fixo não contém apenas uma política forte-cíclica, ele pode incluir ações que não levam para a meta, isto é, ciclos em que todos os estados pertencem a $\mathcal{R}^{*}$. Apesar disso, esse submodelo é o bastante para definir o conjunto de estados que em algum momento alcançam um estado meta. Observe que se assumirmos que o agente sempre tem uma chance de escolher uma ação diferente dentro desse submodelo, então ele em algum momento escolheria uma ação que sai do ciclo e, de forma similar a suposição de equidade, em algum momento alcançaria um estado meta. Para computar uma política forte-cíclica é possível fazer uma nova regressão fraca que só associa uma ação para cada estado.

\subsection{Discussão}

Entre as abordagens de verificação de modelos, temos abordagens enumerativas, que lidam com uma representação explícita do espaço de estados, e abordagens simbólicas, que representam o modelo do mundo de forma fatorada e raciocinam de forma simbólica sobre essa representação.

Neste capítulo apresentamos um algoritmo de verificação de modelos enumerativo para computar o conjunto $\mathcal{R}$. No próximo capítulo, apresentamos uma versão simbólica desse algoritmo. 


\section{Capítulo 6}

\section{Regressão simbólica para detecção de becos-sem-saída}

No capítulo anterior apresentamos um fragmento do algoritmo PACTL (Seção 5.4) que computa o conjunto de estados $\mathcal{R}$ que pode alcançar um estado meta. Esse algoritmo enumera a função de transição, o que pode ser ineficiente. Para contornar esse problema, algoritmos baseados em verificação de modelos simbólica [Fourman, 2000; McMillan, 1992] representam a função de transição de forma implícita, em geral, através de fórmulas proposicionais e podem ser usados para computarmos a regressão fraca (forte ou forte-cíclica) com operações entre fórmulas. Chamaremos esse algoritmo de regressão simbólica fraca. Assim como o algoritmo SPUDD (Seção 2.3) esses métodos se aproveitam da independência entre as variáveis proposicionais para criar uma representação mais compacta dos conjuntos de estados e da função de transição.

Há duas formas de representar uma função de transição utilizando fórmulas proposicionais. A primeira representa a função de transição de maneira implícita (fatorada) [Fourman, 2000; Rintanen, 2008], criando uma fórmula para cada ação, assim como a linguagem de descrição de ações apresentada na Seção 2.4. A segunda cria uma única fórmula para representar a função de transição de forma explícita [Cimatti et al., 1997; McMillan, 1992]. Neste trabalho optamos pela primeira abordagem, pois ela não adiciona uma variável extra para representar as ações nas fórmulas (como a lógica $\alpha$-CTL). Além disso, por manter uma representação implícita das ações essa abordagem pode consumir menos memória.

\subsection{Representação simbólica de estados}

Podemos representar estados e conjuntos de estados através de fórmulas proposicionais. Um estado $s \in \mathcal{S}$ rotulado por um conjunto de proposições atômicas $\mathcal{L}(s) \subseteq \mathbb{P}$, e um conjunto de estados $Y \subseteq \mathcal{S}$, pode ser representado, respectivamente, pelas fórmulas proposicionais:

$$
\xi(s)=\bigwedge_{p \in \mathcal{L}(s)} p \wedge \bigwedge_{p \in \mathbb{P} \backslash \mathcal{L}(s)} \neg p \quad \text { e } \quad \xi(Y)=\bigvee_{s \in Y} \xi(s)
$$

\subsection{Representação simbólica de ações}

Como vimos na Seção 2.4, um problema de planejamento probabilístico pode ser definido por uma linguagem de descrição de ações. Dado um problema de planejamento probabilístico $\mathcal{P}^{\text {prob }}=$ $\left\langle\mathbb{P}, \mathbb{A}, s_{0}, \mathbb{G}\right\rangle$, uma ação probabilística $a \in \mathbb{A}$ é dada pela dupla $\langle$ Precond, Effects $\rangle$ em que Effects é a lista de efeitos da forma $\left[p_{1}:\left(A d d_{1}\right.\right.$, Del $\left._{1}\right), \ldots, p_{n}:\left(A d d_{n}\right.$, Del $\left.\left._{n}\right)\right]$ tal que Precond, $A d d_{1}, \ldots$, Del $_{n}$ são conjuntos de proposições atômicas.

Fourman [2000] propôs uma forma de representar e raciocinar sobre ações determinísticas utilizando operações entre fórmulas proposicionais. Essa representação foi estendida para ações nãodeterminísticas por Menezes et al. [2014]. Neste trabalho, para o cálculo do conjunto $\mathcal{R}$, usando a 
regressão simbólica fraca, de um problema de planejamento probabilístico, ignoramos as probabilidades dos efeitos das ações e representamos uma ação $a \in \mathbb{A}$ pela tupla $\langle$ Precond, Effects, Changes $\rangle$ sendo Changes a lista de proposições que ocorre em Effects.

A representação simbólica de uma ação $a=\langle$ Precond, Effects, Changes $\rangle$ é denotada por $\xi(a)$, sendo os conjuntos Precond e Effects também representados como fórmulas proposicionais, conforme a seguir:

$$
\xi(\text { Precond })=\bigwedge_{p \in \text { Precond }} p \quad \text { e } \quad \xi(\text { Effects })=\bigvee_{1 \leq i \leq n}\left(\bigwedge_{p \in A d d_{i}} p \wedge \bigwedge_{p \in \text {Del}_{i}} \neg p\right) .
$$

\subsection{Operações simbólicas: restrição e quantificação existencial}

Para computarmos o conjunto $\mathcal{R}$ utilizando técnicas de verificação de modelos simbólica, além das operações lógicas básicas de conjunção e disjunção, são necessários os operadores de restrição e quantificação existencial, a saber:

Restrição: Dada uma fórmula proposicional $\phi$, uma proposição $p$ e uma constante $x \in\{\top, \perp\}$, denotamos por $\phi[x / p]$ a fórmula proposicional obtida substituindo todas as ocorrências da proposição $p$ em $\phi$ pela constante $x$. Assim, se $\phi$ descreve um conjunto de estados $Y, \phi[T / p]$ representa o conjunto de estados de Y em que $p$ é verdadeira. De forma análoga, $\phi[\perp / p]$ representa o conjunto de estados de Y em que $p$ é falsa.

Quantificação existencial: Dada uma fórmula proposicional $\phi$ e uma variável proposicional $p$, a quantificação existencial da fórmula $\phi$ sobre os valores da proposição $p$ é:

$$
\exists(p) . \phi=\phi[\perp / p] \vee \phi[T / p] .
$$

Também podemos definir a quantificação existencial para um conjunto de proposições. Seja $\mathcal{P}^{\prime}=\left[p_{1}, p_{2}, \cdots, p_{n}\right]$ um subconjunto das variáveis proposicionais que ocorrem em $\phi$, temos que $\exists B \cdot \phi=\exists\left(p_{1}\right) \cdot \exists\left(p_{2}, \cdots, p_{n}\right) \cdot \phi$.

\subsection{Diagramas de decisão binária}

Diagramas de decisão binária (BDDs) [Bryant, 1986] são utilizados para representar funções proposicionais de forma compacta e realizar operações entre fórmulas proposicionais de maneira eficiente. Denotamos por $\phi^{\mathrm{DD}}$ o BDD da correspondente à fórmula proposicional $\phi$. A Tabela 6.1 mostra a correspondência entre os operadores lógicos utilizados pelo algoritmo de regressão simbólica e as respectivas operações entre BDDs.

\begin{tabular}{|c|c|}
\hline Operadores lógicos & Operações entre BDDs \\
\hline$\phi_{1} \vee \phi_{2}$ & $\phi_{1}^{\mathrm{DD}} \oplus \phi_{1}^{\mathrm{DD}}$ \\
\hline$\phi_{1} \wedge \phi_{2}$ & $\phi_{1}^{\mathrm{DD}} \otimes \phi_{1}^{\mathrm{DD}}$ \\
\hline$\exists B . \phi$ & $\operatorname{exists}\left(B, \phi^{\mathrm{DD}}\right)$ \\
\hline
\end{tabular}

Tabela 6.1: Correspondência entre operadores lógicos e operações entre BDDs.

\subsection{Regressão simbólica fraca: cálculo do conjunto $\mathcal{R}$}

A pré-imagem fraca de um conjunto de estados $Y$ por uma ação $a=\langle$ Precond, Effects, Changes $\rangle$ (chamada regressão por ação), é dado por:

$$
\text { preimage }_{a}(Y)=\xi(\text { Precond }) \wedge \exists(\text { Changes }) \cdot(\xi(\text { Effects }) \wedge \xi(Y)) .
$$


Finalmente, a pré-imagem de um conjunto de ações $\mathbb{A}$, é dado por:

$$
\operatorname{preimage}_{\mathbb{A}}(Y)=\bigvee_{a \in \mathbb{A}} \operatorname{preimage}_{a}(Y) \text {. }
$$

Com essas definições, a implementação simbólica da pré-imagem fraca pode se aproveitar da estrutura fatorada do domínio, e computar $\mathcal{R}$ de forma mais eficiente. $\mathrm{O}$ algoritmo de regressão simbólica fraca (Algoritmo 6.1), utiliza diagramas de decisão binária para representar todas as fórmulas proposicionais [McMillan, 1992].

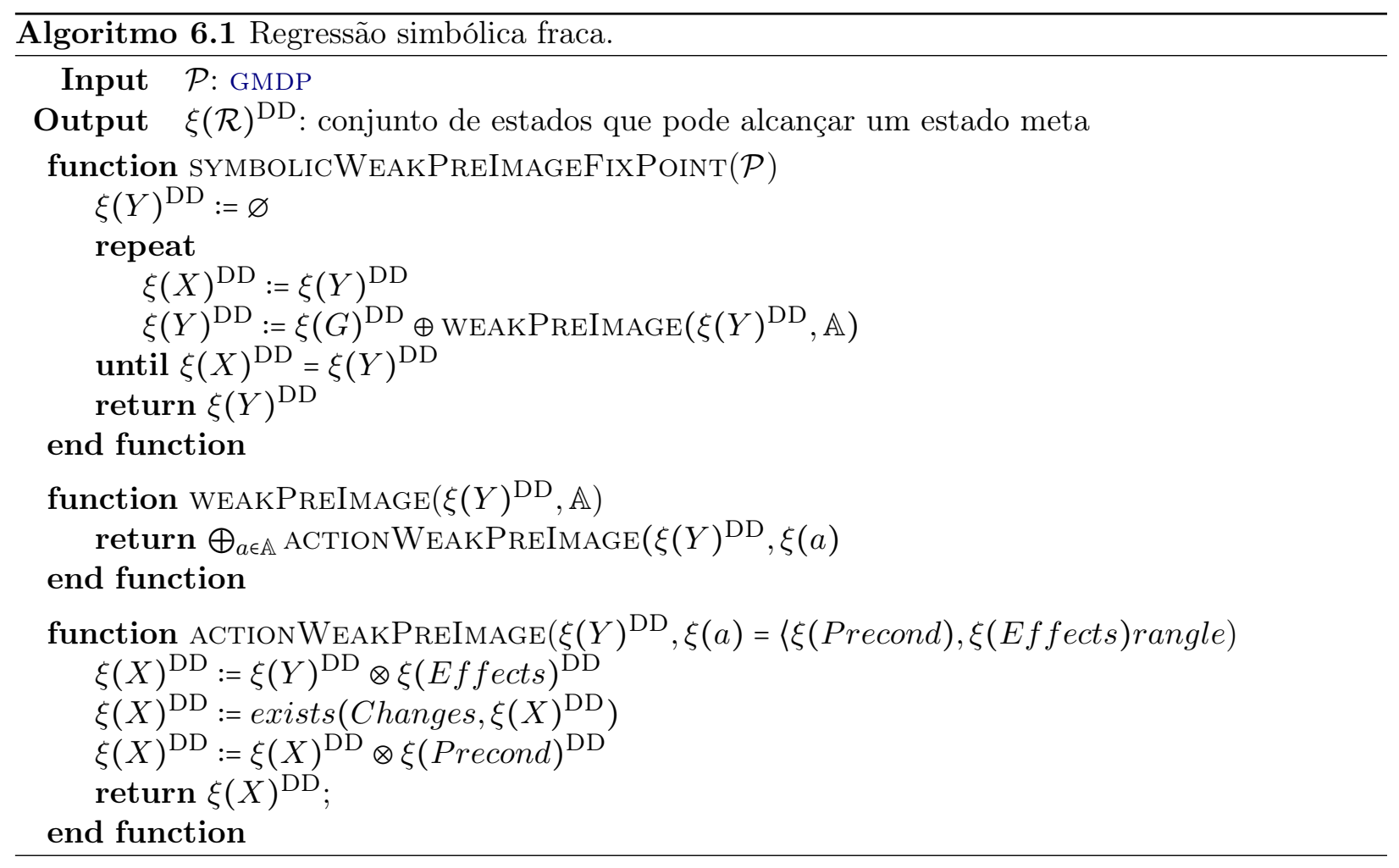

A função WEAKPreImage no Algoritmo 6.1 computa a pré-imagem fraca $\alpha$-CTL de um conjunto de estados $\xi(Y)^{D D}$ dado um conjunto de ações simbólicas $\mathbb{A}$. A função ACTionWeakPreImage implementa a regressão fraca por uma ação $a \in \mathbb{A}$, conforme Equação 6.1. Finalmente, o método SYMBOLICWEAKPreImageFiXPoint computa o ponto fixo da pré-imagem fraca, assim como no Algoritmo 5.1 esse método agrega na variável $Y$ o conjunto de estados que podem alcançar um estado meta.

\subsection{Um novo detector de becos-sem-saída - SWR-DE}

Para resolver GMDPs, o conjunto $\mathcal{R}$ é utilizado para detectar becos-sem-saída simplesmente verificando se $s \notin \mathcal{R}$. Isso também pode ser feito utilizando BDDs conforme a seguir: se $\xi(s) \wedge \xi(\mathcal{R})=\perp$ então $s$ é um beco-sem-saída. No Algoritmo 6.2 a função SWR-DE mostra como isso pode ser feito através de operções entre BDDs. Chamamos o novo método de detector por regressão simbólica fraca (Symbolic Weak Regression Detector - SWR-DE). Note que, o SWR-DE utiliza o Algoritmo 6.1 para computar o conjunto $\mathcal{R}$ antes de iniciar a etapa de planejamento.

O Algoritmo 6.2 mostra como podemos sobrescrever o método Initialize (Algoritmo 2.2) dos algoritmos de planejamento probabilístico, para utilizar o novo método de detecção de becos-semsaída ao inicializar a função valor de um estado $s \in S$. Antes de computar a heurística para o estado $s$, esse método checa se $s$ é um beco-sem-saída (Linha 1), nesse caso, define-se o valor de $s$ como $\infty$ $\left(V^{0}(s)=\infty\right)$. Somente se $s$ pertencer a $\mathcal{R}$, isto é, se o estado não for um beco-sem-saída, o algoritmo computa o valor da heurística para esse estado. 


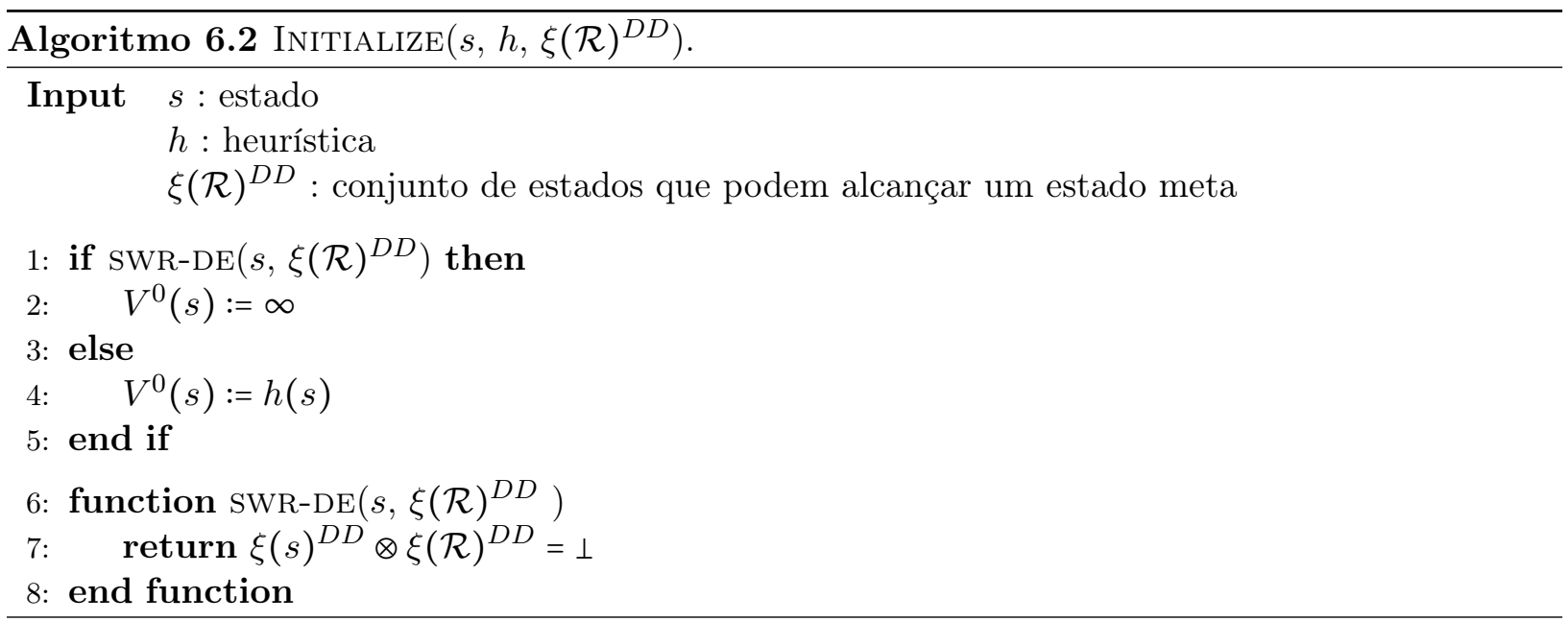

Observamos que as heurísticas de insolubilidade descritas na Seção 4.3 poderiam ser utilizadas de forma similar para inicializar a função valor. No próximo capítulo, avaliamos o comportamento de planejadores probabilísticos que lidam com becos-sem-saída ao utilizarem o método de detecção SWR-DE, proposto nessa seção. 


\section{Capítulo 7}

\section{Análise Empírica}

O algoritmo de regressão simbólica fraca (Algoritmo 6.1), foi implementado no arcabouço de planejamento probabilístico mGPT [Bonet e Geffner, 2005] e integrado à biblioteca de BDDs CUDD [Somenzi, 2015], que contém as operações básicas entre BDDs. O mGPT possui planejadores eficientes, como o LRTDP (Seção 2.2.1) e o HMDPP (Seção 4.2.2), bem como implementa diversas heurísticas que usaremos em nossa análise. Para a integração do algoritmo de deteç̧ão de becos-sem-saída proposto nessa dissertação, o SWR-DE, foi necessário adaptar o mGPT para chamar o novo método de inicialização da função valor (Algoritmo 6.2).

Dividimos os experimentos em duas partes. A primeira parte avalia empiricamente os métodos de detecção de becos-sem-saída, em termos da quantidade de becos-sem-saída detectados no conjunto de estados alcançáveis a partir de $s_{0}$ e do tempo gasto nessa detecção (Seção 7.2). A segunda parte da nossa análise verifica o custo computacional dos algoritmos de planejamento somado ao do método proposto (Seção 7.3). A próxima seção apresenta os domínios benchmark selecionados para essas análises.

\subsection{Domínios}

Para realizar os testes, utilizamos 7 domínios usados nas competições internacionais de planejamento probabilístico ${ }^{1}$ conhecidos por possuírem becos-sem-saída, a saber:

- Navigation_O (SSPUDE MDP);

- Navigation_1 (SSPUDE MDP);

- Navigation_2 (SSPUDE MDP);

- TriangleTireWorld_o (SSPADE MDP);

- TriangleTireWorld_1 (SSPADE MDP);

- Drive (SSPUde MDP); e

- ExplodingBlocksWorld (SSPUDE MDP).

Note que os domínios do tipo SSPUDE MDP não possuem penalidade finita e portanto tratamos todos esses domínios como $i$ SSPUDE MDP.

\subsubsection{Navigation}

O domínio Navigation foi originalmente definido na linguagem RDDL (Relational Dynamic Influence Diagram Language) [Sanner, 2010] para a competição de planejamento probabilístico de 2011 [Sanner e Youn, 2011] (o Apêndice D apresenta a especificação deste domínio em RDDL).

\footnotetext{
${ }^{1}$ icaps-conference.org/index.php/main/competitions
} 
Chamaremos essa extensão de Navigation_0. Neste domínio um robô deve ir de uma localização origem para uma localização destino percorrendo um mapa, ilustrado na Figura 7.1. O robô é capaz de executar quatro ações move-south, move-north, move-west e move-east. Ao aplicar a ação move-north, existe uma chance do robô desaparecer, o que representa um beco-sem-saída, uma vez que o robô não poderá mais se movimentar. Como não existe uma política própria a partir do estado inicial, esse problema pertence à classe dos SSPUDE MDPs.

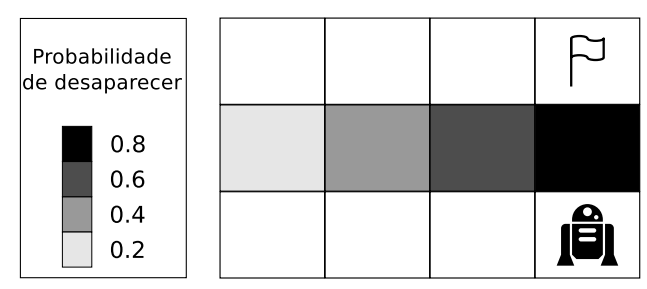

Figura 7.1: Exemplo de problema do dominio Navigation. A tonalidade de cada posição representa a probabilidade do robô desaparecer.

Cada instância desse domínio é parametrizada pelo número total de colunas $(\mathrm{col})$ e pelo número total de linhas (lin). Outros parâmetros são $\min _{p}$ e $\max _{p}$ que definem, respectivamente, as probabilidades mínima e máxima do robô desaparecer ao se mover para o norte. A probabilidade mínima define a probabilidade do robô desaparecer nas posições mais à esquerda (oeste) do mapa e a probabilidade máxima define a probabilidade do robô desaparecer nas posições mais à direita (leste). Assim, a probabilidade do robô desaparecer na coluna $j \in[1, \mathrm{col}]$ é dada por:

$$
\text { probabilidade_desaparecer }(j)=\min _{p}+(j-1) \frac{\left(\max _{p}-\min _{p}\right)}{c o l-1} .
$$

Note que o número de variáveis proposicionais $(|\mathbb{P}|)$ é igual ao número de localizações possíveis do robô num_loc $=c o l * l i n$. Estados em que o robô encontra-se em duas localizações são estados inconsistentes. No entanto, é possível que ele não esteja em nenhuma localização, caso ele desapareça ao se movimentar para o norte. Assim, o número de estados alcançáveis é dado pela quantidade de possíveis localizações do robô mais um (o estado em que ele não está em nenhum lugar).

Observamos que nesses problemas existe um único beco-sem-saída, o estado em que o veículo desapareceu e não se encontra em nenhuma das localizações. Nesse estado, nenhuma ação é aplicável, portanto este estado é um beco-sem-saída explícito. Os demais estados possuem quatro ações aplicáveis. A Tabela 7.1 mostra a análise estatística de 16 instâncias do domínio Navigation.

\begin{tabular}{|l|l|l|r|r|r|r|r|r|}
\hline lin & col & $|\mathbb{P}|$ & $\begin{array}{c}\text { Estados } \\
\text { Alcançáveis }\end{array}$ & Ações & Traps & $\begin{array}{c}\text { Estados } \\
\text { por Trap }\end{array}$ & Becos-sem-saída & $|\mathcal{R}|$ \\
\hline 4 & 3 & 12 & 13 & 4 & 1 & 1 & 1 & 12 \\
4 & 6 & 24 & 25 & 4 & 1 & 1 & 1 & 24 \\
4 & 9 & 36 & 37 & 4 & 1 & 1 & 1 & 36 \\
4 & 12 & 48 & 49 & 4 & 1 & 1 & 1 & 48 \\
4 & 15 & 60 & 61 & 4 & 1 & 1 & 1 & 60 \\
4 & 18 & 72 & 73 & 4 & 1 & 1 & 1 & 72 \\
4 & 21 & 84 & 85 & 4 & 1 & 1 & 1 & 84 \\
4 & 24 & 96 & 97 & 4 & 1 & 1 & 1 & 96 \\
4 & 27 & 108 & 109 & 4 & 1 & 1 & 1 & 108 \\
4 & 30 & 120 & 121 & 4 & 1 & 1 & 1 & 120 \\
4 & 33 & 132 & 133 & 4 & 1 & 1 & 1 & 132 \\
4 & 36 & 144 & 145 & 4 & 1 & 1 & 1 & 144 \\
4 & 39 & 156 & 157 & 4 & 1 & 1 & 1 & 156 \\
4 & 42 & 168 & 169 & 4 & 1 & 1 & 1 & 168 \\
4 & 45 & 180 & 181 & 4 & 1 & 1 & 1 & 180 \\
4 & 48 & 192 & 193 & 4 & 1 & 1 & 1 & 192 \\
\hline
\end{tabular}

Tabela 7.1: Estatísticas para 16 instâncias do dominio Navigation_0. 
Navigation (extensão 1). Como todas as instâncias do domínio Navigation possuem apenas um beco-sem-saída que pode ser detectado trivialmente, esse domínio não é interessante para avaliarmos a capacidade dos métodos de deteç̧ão de becos-sem-saída. Assim, estendemos esse domínio adicionando um objeto que deve ser levado pelo agente da localização inicial para a localização meta. Chamaremos essa extensão de Navigation_1. Nesse caso, ao invés de desaparecer, o agente derruba o objeto e como não existe uma ação para pegar novamente o objeto, esse é um beco-sem-saída. Uma vez que o robô pode continuar se movimentando normalmente, cria-se uma trap permanente com num_loc estados, uma para cada posição em que o objeto é derrubado (para isso foi adicionado o predicado object-at (loc) que representa a posição em que o objeto foi derrubado). Com isso criamos num_loc traps permanentes com num_loc estados em cada trap, como mostra a Tabela 7.2 .

\begin{tabular}{|c|c|c|c|c|c|c|c|c|}
\hline $\operatorname{lin}$ & $\mathrm{col}$ & $|\mathbb{P}|$ & $\begin{array}{l}\text { Estados } \\
\text { Alcançáveis }\end{array}$ & Ações & Traps & $\begin{array}{l}\text { Estados } \\
\text { por Trap }\end{array}$ & Becos-sem-saída & $|\mathcal{R}|$ \\
\hline 4 & 3 & 25 & 156 & 4 & 12 & 12 & 144 & 12 \\
\hline 4 & 6 & 49 & 600 & 4 & 24 & 24 & 576 & 24 \\
\hline 4 & 9 & 73 & 1332 & 4 & 36 & 36 & 1296 & 36 \\
\hline 4 & 12 & 97 & 2352 & 4 & 48 & 48 & 2304 & 48 \\
\hline 4 & 15 & 121 & 3660 & 4 & 60 & 60 & 3600 & 60 \\
\hline 4 & 18 & 145 & 5256 & 4 & 72 & 72 & 5184 & 72 \\
\hline 4 & 21 & 169 & 7140 & 4 & 84 & 84 & 7056 & 84 \\
\hline 4 & 24 & 193 & 9312 & 4 & 96 & 96 & 9216 & 96 \\
\hline 4 & 27 & 217 & 11772 & 4 & 108 & 108 & 11664 & 108 \\
\hline 4 & 30 & 241 & 14520 & 4 & 120 & 120 & 14400 & 120 \\
\hline 4 & 33 & 265 & 17556 & 4 & 132 & 132 & 17424 & 132 \\
\hline 4 & 36 & 289 & 20880 & 4 & 144 & 144 & 20736 & 144 \\
\hline 4 & 39 & 313 & 24492 & 4 & 156 & 156 & 24336 & 156 \\
\hline 4 & 42 & 337 & 28392 & 4 & 168 & 168 & 28224 & 168 \\
\hline 4 & 45 & 361 & 32580 & 4 & 180 & 180 & 32400 & 180 \\
\hline 4 & 48 & 385 & 37056 & 4 & 192 & 192 & 36864 & 192 \\
\hline
\end{tabular}

Tabela 7.2: Estatísticas para 16 instâncias do domínio Navigation_1.

Navigation (extensão 2). Uma outra extensão do Navigation foi criada em que o robô deve levar dois objetos (object 1 e ob ject2) para a localização de destino. Chamaremos essa extensão de Navigation_2. Nessa extensão, criamos a ação grab (obj) e o agente pode tentar recuperar um dos objetos depois que ele foi derrubado, porém para que o domínio mantenha suas características em relação aos becos-sem-saída, a ação grab derruba o outro objeto e assim o agente nunca consegue atingir um estado meta se um dos objetos for derrubado. Essa ação é interessante pois cria uma relação de exclusão mútua entre hold-object 1 e hold-object2, depois que um dos objetos é derrubado.

Note que, comparando os 3 domínios (Tabelas 7.1, 7.2 e 7.3) em termos do tamanho de $\mathcal{R}$ (estados alcançáveis a partir dos quais é possível alcançar a meta) se mantém constante para todas as instâncias. Assim, as extensões propostas mantêm o tamanho de $\mathcal{R}$ e aumenta o número de becos-sem-saída.

\subsubsection{Triangle Tire World}

O domínio TireWorld, proposto para a IPPC- $2004^{2}$, também é um problema clássico de planejamento com becos-sem-saída (o Apêndice D apresenta a especificação deste domínio em PPDDL). Chamaremos esse domínio de TriangleTireWorld_0. Nesse problema, um veículo pode se mover entre diferentes localizações através de estradas direcionadas, com o objetivo de sair de uma localização inicial $l o c_{0}$ e chegar a uma localização meta $l o c_{g}$. Entretanto, a cada movimentação existe uma probabilidade de furar um pneu. Neste caso, o veículo não pode se movimentar, no entanto, é

\footnotetext{
${ }^{2}$ Competição Internacional de Planejamento Probabilístico de 2004 (http://www.cs.rutgers.edu/ mlittman/ topics/ipc04-pt/).
} 


\begin{tabular}{|l|l|r|r|r|r|r|r|r|}
\hline lin & col & $|\mathbb{P}|$ & $\begin{array}{c}\text { Estados } \\
\text { Alcançáveis }\end{array}$ & Ações & Traps & $\begin{array}{r}\text { Estados } \\
\text { por Trap }\end{array}$ & Becos-sem-saída & $|\mathcal{R}|$ \\
\hline 4 & 3 & 37 & 300 & 5 & 12 & 24 & 288 & 12 \\
4 & 6 & 73 & 1176 & 6 & 24 & 48 & 1152 & 24 \\
4 & 9 & 109 & 2628 & 7 & 36 & 72 & 2592 & 36 \\
4 & 12 & 145 & 4656 & 8 & 48 & 96 & 4608 & 48 \\
4 & 15 & 181 & 7260 & 9 & 60 & 120 & 7200 & 60 \\
4 & 18 & 217 & 10440 & 10 & 72 & 144 & 10368 & 72 \\
4 & 21 & 253 & 14196 & 11 & 84 & 168 & 14112 & 84 \\
4 & 24 & 289 & 18528 & 12 & 96 & 192 & 18432 & 96 \\
4 & 27 & 325 & 23436 & 13 & 108 & 216 & 23328 & 108 \\
4 & 30 & 361 & 28920 & 14 & 120 & 240 & 28800 & 120 \\
4 & 33 & 397 & 34980 & 15 & 132 & 264 & 34848 & 132 \\
4 & 36 & 433 & 41616 & 16 & 144 & 288 & 41472 & 144 \\
4 & 39 & 469 & 48828 & 17 & 156 & 312 & 48672 & 156 \\
4 & 42 & 505 & 56616 & 18 & 168 & 336 & 56448 & 168 \\
4 & 45 & 541 & 64980 & 19 & 180 & 360 & 64800 & 180 \\
4 & 48 & 577 & 73920 & 20 & 192 & 384 & 73728 & 192 \\
\hline
\end{tabular}

Tabela 7.3: Estatísticas para 16 instâncias do domínio Navigation_2.

possível trocar o pneu furado se o veículo estiver em uma localização que tem um estepe. Assim, estados em que um pneu está furado, e não existe um pneu novo na localização em que o veículo se encontra, são becos-sem-saída. Little e Thiebaux [2007] propuseram uma extensão para esse domínio, o Triangle TireWorld, em que a cada instância o mapa cresce de forma controlada mantendo a formação de um triângulo. Nessa extensão, cada instância possui apenas uma rota em que todas as posições possuem estepes, logo existe uma política $s_{0}$-própria e todas as instâncias desse domínio são SSPADE MDPs. A Figura 7.2 mostra duas instâncias do domínio TriangleTireWorld_o.
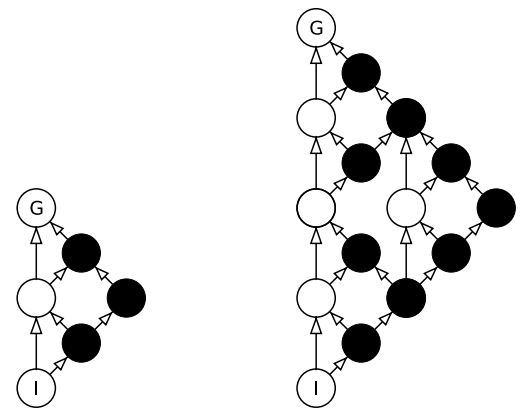

Figura 7.2: Duas instâncias do dominio Triangle TireWorld. A localização rotulada por I indica a localização inicial do veículo e a por $G$, a localização meta. Círculos pretos indicam localizações que possuem estepe. Uma aresta direcionada entre duas localizações representa uma estrada e seu sentido.

A Tabela 7.4 mostra como o número de estados alcançáveis cresce exponencialmente neste domínio (calculados empiricamente). Observamos ainda que o número médio de ações aplicáveis em cada estado é menor que 1, pois becos-sem-saída não possuem ações aplicáveis.

\begin{tabular}{|l|r|r|r|}
\hline Instância & $|\mathbb{P}|$ & $\begin{array}{c}\text { Estados } \\
\text { Alcançáveis }\end{array}$ & $\begin{array}{c}\text { Número Médio de Ações } \\
\text { Aplicáveis por Estado }\end{array}$ \\
\hline 1 & 19 & 42 & 0.857143 \\
2 & 51 & 946 & 0.849894 \\
3 & 99 & 19562 & 0.830181 \\
4 & 163 & 384354 & 0.819193 \\
5 & 243 & 7258714 & 0.812965 \\
\hline
\end{tabular}

Tabela 7.4: Estatísticas de 5 instâncias do domínio TriangleTireWorld_0. 
Triangle Tire World (extensão). Observando que os becos-sem-saída do Triangle TireWorld são facilmente detectáveis, criamos uma extensão para esse problema, em que um passageiro (que fará a troca do pneu furado) pode entrar e sair do carro. Nesse caso, ele só pode trocar o pneu se estiver fora do carro e o veículo só pode se mover se o passageiro estiver dentro do carro. Chamaremos essa extensão de TriangleTireWorld_1. Dessa forma, os becos-sem-saída não são explícitos pois todos os estados têm ao menos uma ação aplicável (entrar ou sair do carro) que muda o estado atual, logo, o número médio de ações aplicáveis em cada estado é maior que 1, conforme mostra a

\begin{tabular}{|l|r|r|r|}
\hline Instância & $|\mathbb{P}|$ & $\begin{array}{c}\text { Estados } \\
\text { Alcançáveis }\end{array}$ & $\begin{array}{c}\text { Número Médio de Ações } \\
\text { Aplicáveis por Estado }\end{array}$ \\
\hline 1 & 21 & 84 & 1.42857 \\
2 & 53 & 1892 & 1.42495 \\
3 & 101 & 39124 & 1.41509 \\
4 & 165 & 768708 & 1.40960 \\
5 & 245 & 14517428 & 1.40648 \\
\hline
\end{tabular}

Tabela 7.5: Estatísticas de 5 instâncias do domínio TriangleTireWorld_1.

Tabela 7.5. Note que, nesta extensão o número de estados alcançáveis dobra devido a adição das proposições que indicam se o passageiro está dentro ou fora do veículo.

\subsubsection{Drive}

No domínio Drive, proposto para a IPPC-2006 ${ }^{3}$, também temos um veículo que se move em uma rede de vias e semáforos. Ao se movimentar pelas vias o agente pode morrer, o que constitui um becosem-saída. A probabilidade do agente morrer depende do sentido em que o veículo está dirigindo, do comprimento dos segmentos, da cor em que os semáforos se encontram e da preferência entre as vias. Caso o semáforo esteja vermelho o agente pode esperar que a cor mude ou avançar. Todas as movimentações possuem alguma probabilidade de alcançar um beco-sem-saída, dessa forma as instâncias desse domínio são SSPUDE MDPs. A Tabela 7.6 mostra as estatísticas de 15 instâncias do domínio Drive.

\begin{tabular}{|l|r|r|r|}
\hline Instância & $|\mathbb{P}|$ & $\begin{array}{c}\text { Estados } \\
\text { Alcançáveis }\end{array}$ & $\begin{array}{c}\text { Número Médio de Ações } \\
\text { Aplicáveis por Estado }\end{array}$ \\
\hline 1 & 12.0 & 53.0 & 0.679245 \\
2 & 17.0 & 89.0 & 0.741573 \\
3 & 17.0 & 89.0 & 0.741573 \\
4 & 17.0 & 149.0 & 0.805369 \\
5 & 17.0 & 149.0 & 0.805369 \\
6 & 24.0 & 293.0 & 0.860068 \\
7 & 33.0 & 377.0 & 0.875332 \\
8 & 33.0 & 377.0 & 0.875332 \\
9 & 44.0 & 593.0 & 0.900506 \\
10 & 44.0 & 725.0 & 0.910345 \\
11 & 57.0 & 1013.0 & 0.923988 \\
12 & 57.0 & 1013.0 & 0.923988 \\
13 & 72.0 & 1349.0 & 0.934025 \\
14 & 89.0 & 1733.0 & 0.941720 \\
15 & 108.0 & 2165.0 & 0.947806 \\
\hline
\end{tabular}

Tabela 7.6: Estatísticas de 15 instâncias do domínio Drive.

\subsubsection{Exploding Blocks World}

O domínio ExplodingBlocksWorld, também proposto para a IPPC-2004, é uma extensão do domínio BlocksWorld (Mundo dos Blocos) em que os blocos podem explodir. Assim, como no

\footnotetext{
${ }^{3}$ Competição Internacional de Planejamento Probabilístico de 2006 (http://idm-lab.org/wiki/icaps/ipc2006/ probabilistic/).
} 
domínio original, o agente é capaz de pegar os blocos e colocar sobre outros blocos ou na mesa, e o objetivo é colocar os blocos em uma determinada configuração. Quando um bloco A é colocado sobre outro bloco B, existe uma probabilidade do bloco A explodir o bloco B (dizemos que o bloco A foi detonado e o bloco $\mathrm{B}$ foi destruído). Um bloco detonado não pode ser detonado novamente mas pode ser movimentado. Blocos destruídos não podem ser movimentados, por isso, um estado com um bloco destruído que não está na posição da meta é um beco-sem-saída. As instâncias desse domínio podem ser tanto SSPADE MDPs quanto SSPUDE MDPs, dependendo da configuração inicial dos blocos e da meta (todas as instâncias avaliadas são SSPUDE MDPs). A Tabela 7.7 mostra as estatísticas de 4 instâncias do domínio ExplodingBlocksWorld.

\begin{tabular}{|l|r|r|r|}
\hline Instância & $|\mathbb{P}|$ & $\begin{array}{c}\text { Estados } \\
\text { Alcançáveis }\end{array}$ & $\begin{array}{c}\text { Número Médio de Ações } \\
\text { Aplicáveis por Estado }\end{array}$ \\
\hline 1 & 52 & 195083 & 1.60970 \\
2 & 52 & 195083 & 1.60970 \\
3 & 68 & 4493131 & 1.70423 \\
4 & 68 & 4493131 & 1.70423 \\
\hline
\end{tabular}

Tabela 7.7: Estatísticas de 4 instâncias do domínio ExplodingBlocksWorld.

\subsection{Completude da detecção de becos-sem-saída}

Como dissemos na Seção 4.3, heurísticas de planejamento probabilístico podem ser usadas tanto para estimar o valor inicial de um estado $s(V(s))$ como para detectar se $s$ é um beco-sem-saída (caso em que elas devolvem um valor infinito). Porém, em geral, as heurísticas não são capazes de detectar todos os becos-sem-saída de um problema, a não ser a heurística $h_{m-m}$, porém com alto custo computacional, conforme mostraremos. Para fazer essa análise de completude na detecção de becos-sem-saída, usamos o método generate_state_space do mGPT para gerar todos os estados alcançáveis de cada instância a partir de $s_{0}$, denotado por $\operatorname{Alc}\left(s_{0}\right)$ (esse método faz parte do algoritmo vi do mGPT). A análise foi realizada através de duas medidas: (1) o número de becos-sem-saída detectados no conjunto de estados $A l c\left(s_{0}\right)$; e (2) tempo gasto para avaliar todos os estados de $A l c\left(s_{0}\right)$. Os experimentos foram executados em uma máquina Linux com um processador de $2.53 \mathrm{Ghz}$ e um tempo limite de 10 minutos para cada heurística avaliar todo o espaço de estados.

Foram avaliadas as heurísticas de planejamento clássico: $h_{F F}, h_{a d d}, h_{1}\left(h_{m} \operatorname{com} m=1\right)$ e $h_{2}$ ( $h_{m}$ com $m=2$ ), $p d b$ e $h_{m-m}$ (Capítulo 4). A heurística $h_{m-m}$ foi avaliada utilizando uma busca IDA* equipada com cada uma das heurísticas de planejamento clássico (denotamos por $h \mid h_{m-m}$ quando a busca da heurística $h_{m-m}$ usa uma certa heurística de planejamento clássico $h$ ). No total, analisamos os domínios em 10 heurísticas, mais o método proposto SWR-DE.

\subsubsection{Detecção de becos-sem-saída no domínio Navigation}

Navigation_0. Nesse domínio foram geradas 16 instâncias com 4 linhas, variando o número de colunas de 3 a 48 . Como dissemos anteriormente, no domínio Navigation_o (especificação original) cada instância só possui um beco-sem-saída (explícito). Todas as heurísticas que completaram a avaliação de $A l c\left(s_{0}\right)$ e o método SWR-DE detectaram o beco-sem-saída (estado em que o robô desaparece), para todas as instâncias.

A Figura 7.3 mostra o tempo gasto pelas heurísticas ao avaliar todo o espaço de estados de cada instância do domínio Navigation_0. Podemos observar que as heurísticas $h_{2}$ e $h_{2} \mid h_{m-m}$ consomem muito tempo, não conseguindo avaliar as maiores instâncias dentro do tempo limite estabelecido. As heurísticas $p d b$ e $p d b \mid h_{m-m}$ também consomem muito tempo, alcançando a ordem de centenas de segundos, mas resolveram todas as instâncias. Isso ocorre pois o tempo de pré-processamento da heurística $p d b$ é dominante (note que no gráfico as curvas das duas heurísticas chegam a se sobrepor). A heurística $h_{1} \mid h_{m-m}$ e o método SWR-DE resolveram todos os problemas em aproximadamente 1 


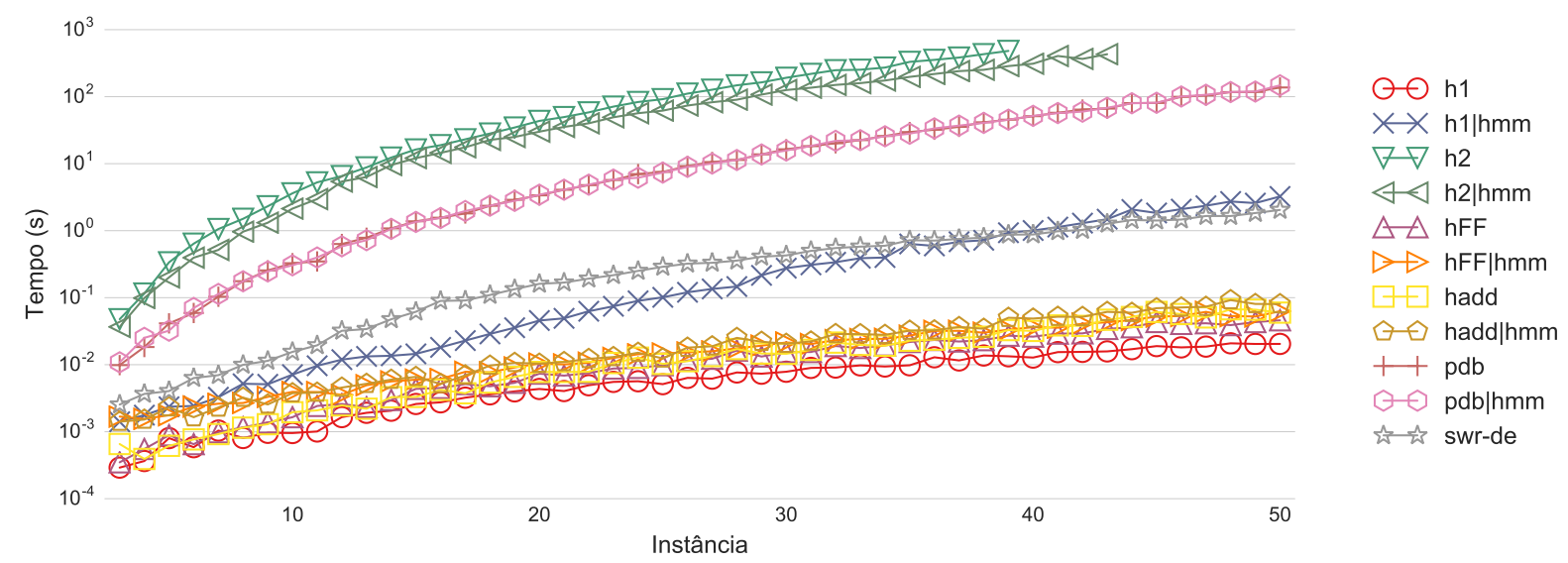

Figura 7.3: Tempo gasto para avaliar todos os estados de 16 instâncias do domínio Navigation_o com quatro linhas. Cada instância é representada pelo número de colunas.

segundo. As demais heurísticas conseguiram avaliar todo o espaço de estados de todas as instâncias em centésimos de segundos.

Navigation_1. Foram geradas as mesmas 16 instâncias, porém, introduzindo o objeto e os fluentes que denotam sua posição hold-object e object-at (loc). No domínio Navigation_1, apesar dos becos-sem-saída possuírem ações aplicáveis e o número de becos-sem-saída ser maior, os resultados em relação a completude da detecção de becos-sem-saída foram iguais aos resultados no domínio Navigation_0. No entanto, conforme mostra a Figura 7.4, o desempenho em tempo foi diferente. As heurísticas $h_{2}$ e $h_{2} \mid h_{m-m}$ só conseguem resolver as instâncias menores. Por outro lado, as heurísticas $p d b$ e $p d b \mid h_{m-m}$ não conseguem resolver as instâncias maiores. As heurísticas $h_{1}$ e $h_{1} \mid h_{m-m}$ resolveram todas as instâncias, mas nesse problema gastaram dezenas de segundos nas instâncias maiores. As demais heurísticas e o método SWR-DE conseguiram avaliar todas as instâncias corretamente em cerca de 1 segundo, sendo que o SWR-DE ficou entre as heurísticas de menor tempo.

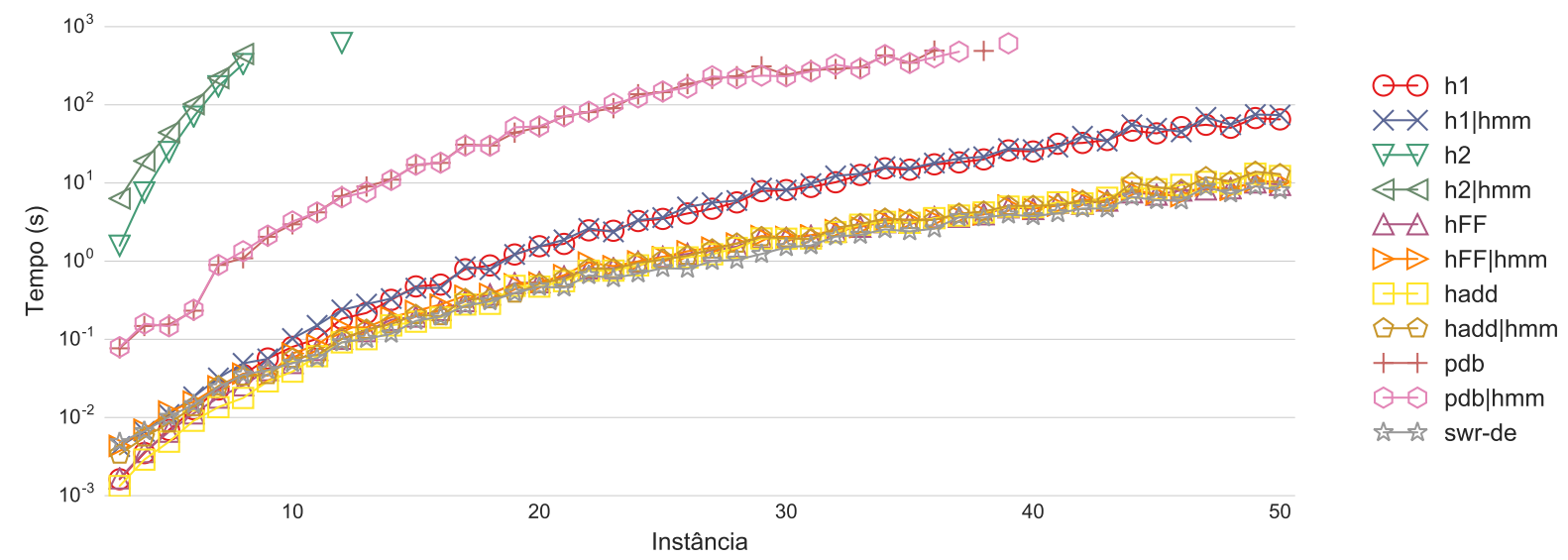

Figura 7.4: Tempo gasto para avaliar todos os estados em 16 instâncias do domínio Navigation_1 com quatro linhas. Cada instância é representada pelo número de colunas.

Navigation_2. Nesse domínio também foram analisadas 16 instâncias, porém com a adição de dois objetos e a ação grab (obj). Podemos observar na Tabela 7.8 que as heurísticas $h_{F F}, h_{a d d}$, $h_{1}$ não foram capazes de detectar becos-sem-saída em nenhuma das instâncias. Conforme vimos no Capítulo 4, essas heurísticas assumem que as proposições atômicas são independentes, pois ignoram os efeitos $D e l$ e assim não percebem a exclusão mútua entre as proposições da meta quando o objeto 
é derrubado. Por outro lado, as heurísticas $h_{2}$ e $h_{2} \mid h_{m-m}$, que consideram conjuntos de proposições de tamanho 2, são capazes de detectar os becos-sem-saída desse domínio. Porém, por consumirem muito tempo em sua análise (Figura 7.5), elas resolveram apenas a instância 3 do Navigation_2. Observamos ainda que outras heurísticas que usam $h_{m-m}$ e $p d b$ detectaram todos os becos-sem-saída em mais instâncias, porém, somente o método de detecção SWR-DE conseguiu detectar todos os becos-sem-saída, inclusive nas instâncias maiores. Isso se deu porque o método de detecção sWRDE foi o mais eficiente, conseguindo avaliar as instâncias mais difíceis em uma ordem de dezenas de segundos.

\begin{tabular}{|l|r|r|r|r|r|r|r|r|r|r|r|r|r|}
\hline Instância & $h_{1}$ & $h_{1} \mid h_{m-m}$ & $h_{2}$ & $h_{2} \mid h_{m-m}$ & $h_{F F}$ & $h_{F F} \mid h_{m-m}$ & $h_{\text {add }}$ & $h_{\text {add }} \mid h_{m-m}$ & $p d b$ & $p d b \mid h_{m-m}$ & SWR-DE \\
\hline 3 & 0 & 288 & 288 & 288 & 0 & 288 & 0 & 288 & 288 & 288 & 288 \\
6 & 0 & 1152 & - & - & 0 & 1152 & 0 & 1152 & 1152 & 1152 & 1152 \\
9 & 0 & 2592 & - & - & 0 & 2592 & 0 & 2592 & 2592 & 2592 & 2592 \\
12 & 0 & - & - & - & 0 & - & 0 & - & 4608 & 4608 & 4608 \\
15 & 0 & - & - & - & 0 & - & 0 & - & 7200 & 7200 & 7200 \\
18 & 0 & - & - & - & 0 & - & 0 & - & 10368 & 10368 & 10368 \\
21 & 0 & - & - & - & 0 & - & 0 & - & 14112 & 14112 & 14112 \\
24 & 0 & - & - & - & 0 & - & 0 & - & 18432 & 18432 & 18432 \\
27 & 0 & - & - & - & 0 & - & 0 & - & - & - & 23328 \\
30 & 0 & - & - & - & 0 & - & 0 & - & - & - & 28800 \\
33 & 0 & - & - & - & 0 & - & 0 & - & - & - & 34848 \\
36 & 0 & - & - & - & 0 & - & 0 & - & - & - & 41472 \\
39 & 0 & - & - & - & 0 & - & 0 & - & - & - & 48672 \\
42 & 0 & - & - & - & 0 & - & 0 & - & - & - & 56448 \\
45 & 0 & - & - & - & 0 & - & 0 & - & - & - & 64800 \\
48 & 0 & - & - & - & 0 & - & 0 & - & - & - & 73728 \\
\hline
\end{tabular}

Tabela 7.8: Número de becos-sem-saída detectados por cada heurística e pelo SWR-DE em 16 instâncias do domínio Navigation_2. Cada instância é representada pelo número de colunas. (O simbolo "." indica Time Out.)

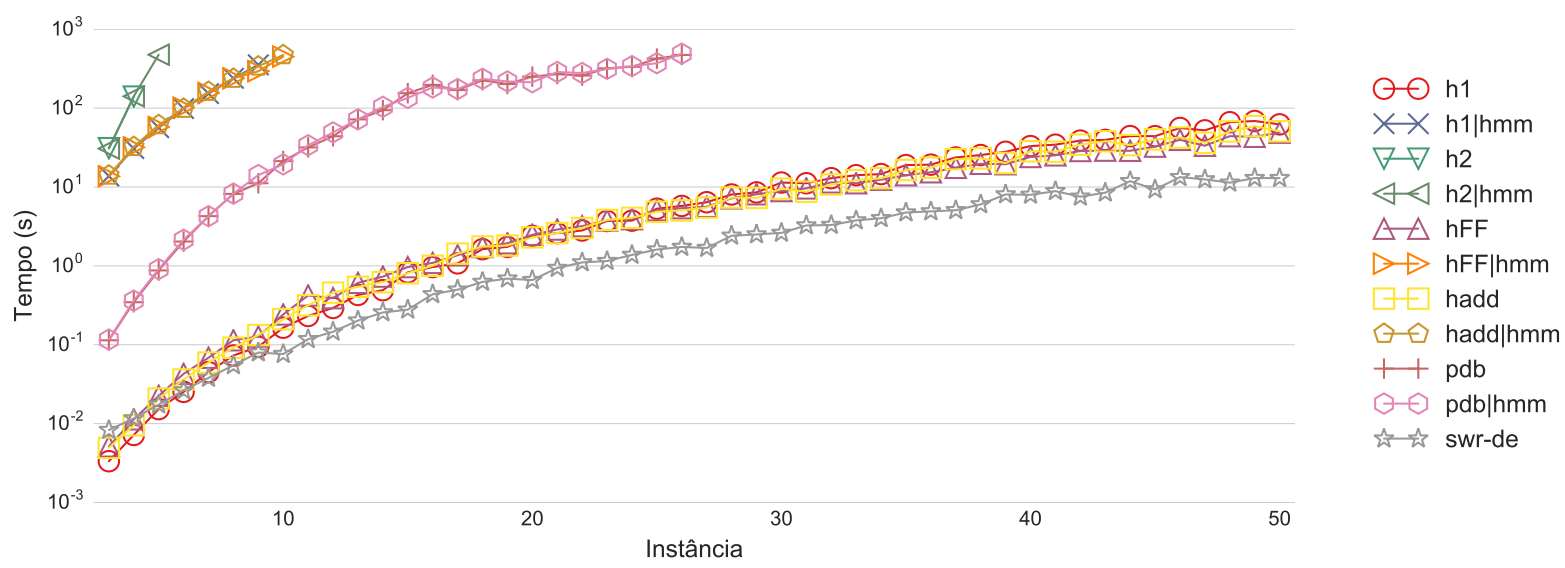

Figura 7.5: Tempo gasto para avaliar todos os estados em 16 instâncias do domínio Navigation_2 com quatro linhas. Cada instância é representada pelo número de colunas.

Comparando o desempenho do método SWR-DE nas 2 extensões do domínio Navigation (Figuras 7.4 e 7.5) percebemos que o tempo desse método não sofreu alterações (aproximadamente 10 segundos), mesmo com o aumento do número de becos-sem-saída. Notamos assim, que o tempo de execução desse método tem uma alta correlação com o tamanho do conjunto $\mathcal{R}$ de estados que podem alcançar um estado meta.

\subsubsection{Detecção de becos-sem-saída no domínio Triangle Tire World}

Triangle TireWorld_0. Conforme mostra a Tabela 7.4 e 7.5 o número de estados alcançáveis no domínio TriangleTireWorld_o cresce exponencialmente. Assim foram avaliadas 5 instâncias, 
com $|\mathbb{P}|=6$ até $|\mathbb{P}|=243$ (não testamos a heurística $h_{2}$ pois ela consome muito tempo, conforme vimos nos experimentos do domínio Navigation). Conforme mostra a Tabela 7.9 todas as heurísticas conseguiram detectar todos os becos-sem-saída em todas as instâncias desse domínio. Isso ocorre, pois, assim como no domínio Navigation_0, não há ações aplicáveis nos becos-sem-saída desse domínio. Note que, conforme mostra a Figura 7.6 o método swR-DE foi o que consumiu menos tempo para avaliar a quinta instância.

\begin{tabular}{|l|r|r|r|r|r|r|r|r|r|}
\hline Instância & $h_{1}$ & $h_{1} \mid h_{m-m}$ & $h_{F F}$ & $h_{F F} \mid h_{m-m}$ & $h_{\text {add }}\left|h_{\text {add }}\right| h_{m-m}$ & $p d b$ & $p d b \mid h_{m-m}$ & SWR-DE \\
\hline 1 & 2 & 2 & 2 & 2 & 2 & 2 & 2 & 2 & 2 \\
2 & 34 & 34 & 34 & 34 & 34 & 34 & 34 & 34 & 34 \\
3 & 462 & 462 & 462 & 462 & 462 & 462 & 462 & 462 & 462 \\
4 & 5978 & 5978 & 5978 & 5978 & 5978 & 5978 & 5978 & 5978 & 5978 \\
5 & 77158 & 77158 & 77158 & 77158 & 77158 & 77158 & 77158 & - & 77158 \\
\hline
\end{tabular}

Tabela 7.9: Número de becos-sem-saída detectados por cada heurística e pelo SWR-DE no dominio TriangleTireWorld_o.

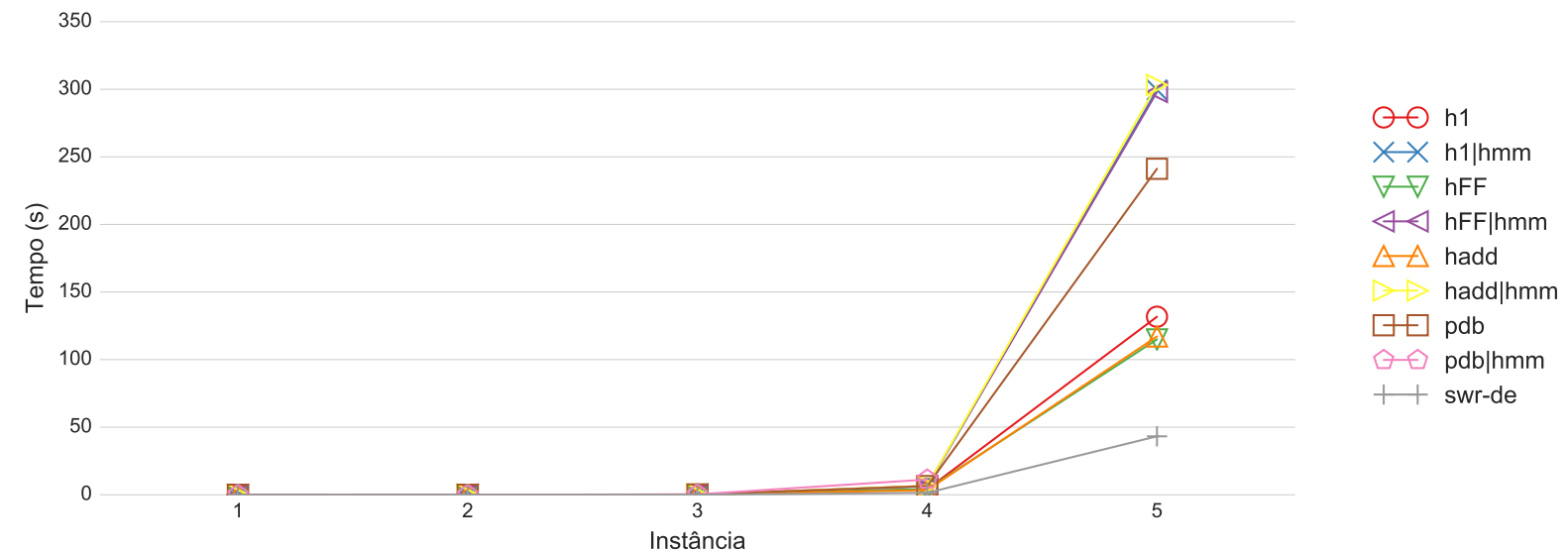

Figura 7.6: Tempo gasto para avaliar todos os estados em 5 instâncias do domínio TriangleTireWorld_0.

Triangle Tire World_1. A Tabela 7.10 mostra que os resultados no domínio Triangle Tire World_1 foram muito parecidos ao do domínio TriangleTireWorld_0. Note que, apenas o método SWR-DE conseguiu avaliar todo o conjunto de estados alcançáveis da última instância.

\begin{tabular}{|l|r|r|r|r|r|r|r|r|r|}
\hline Instância & $h_{1}$ & $h_{1} \mid h_{m-m}$ & $h_{F F}$ & $h_{F F} \mid h_{m-m}$ & $h_{\text {add }}\left|h_{\text {add }}\right| h_{m-m}$ & $p d b$ & $p d b \mid h_{m-m}$ & sWR-DE \\
\hline 1 & 4 & 4 & 4 & 4 & 4 & 4 & 4 & 4 & 4 \\
2 & 68 & 68 & 68 & 68 & 68 & 68 & 68 & 68 & 68 \\
3 & 924 & 924 & 924 & 924 & 924 & 924 & 924 & 924 & 924 \\
4 & 11956 & 11956 & 11956 & 11956 & 11956 & 11956 & 11956 & 11956 & 11956 \\
5 & - & - & - & - & - & - & - & - & 154316 \\
\hline
\end{tabular}

Tabela 7.10: Número de becos-sem-saída detectados por cada heurística e pelo SWR-DE no dominio TriangleTire World_1. 


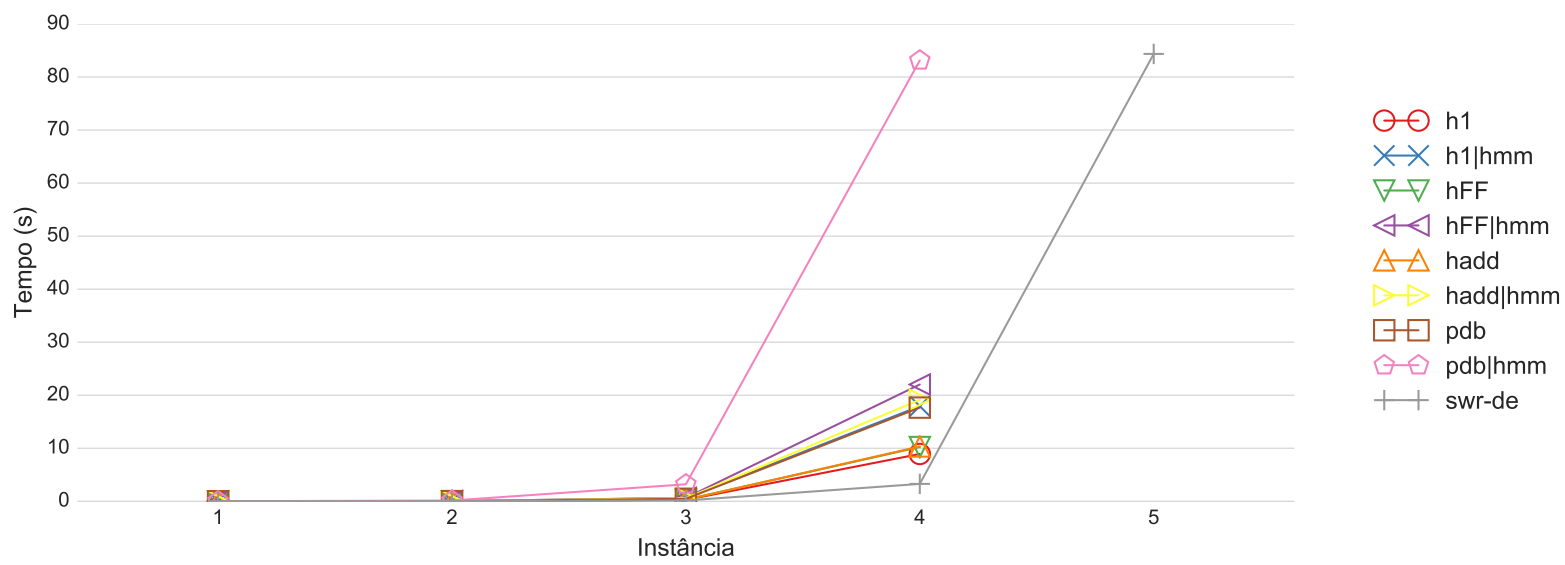

Figura 7.7: Tempo gasto para avaliar todos os estados em 5 instâncias do dominio TriangleTire World_1.

\subsubsection{Detecção de becos-sem-saída no domínio Drive}

No domínio Drive foram testadas todas as 15 instâncias da competição. A busca realizada pela heurística $h_{m-m}$ apresentou um erro neste domínio, por isso seus resultados foram omitidos. Na Tabela 7.11 observamos que todas as heurísticas avaliadas e o método SWR-DE conseguiram detectar todos os becos-sem-saída em todas as instâncias desse domínio. A Figura 7.8 mostra que todos os métodos conseguiram avaliar todas as instâncias dentro do tempo limite, sendo que o método SWR-DE e a heurística $h_{1}$ resolveram a última instância na ordem de segundos, enquanto as demais atingiram a ordem de dezenas de segundos.

\begin{tabular}{|l|r|r|r|r|r|}
\hline Instância & $h_{1}$ & $h_{F F}$ & $h_{a d d}$ & $p d b$ & SWR-DE \\
\hline 1 & 26 & 26 & 26 & 26 & 26 \\
2 & 44 & 44 & 44 & 44 & 44 \\
3 & 44 & 44 & 44 & 44 & 44 \\
4 & 74 & 74 & 74 & 74 & 74 \\
5 & 74 & 74 & 74 & 74 & 74 \\
6 & 146 & 146 & 146 & 146 & 146 \\
7 & 188 & 188 & 188 & 188 & 188 \\
8 & 188 & 188 & 188 & 188 & 188 \\
9 & 296 & 296 & 296 & 296 & 296 \\
10 & 362 & 362 & 362 & 362 & 362 \\
11 & 506 & 506 & 506 & 506 & 506 \\
12 & 506 & 506 & 506 & 506 & 506 \\
13 & 674 & 674 & 674 & 674 & 674 \\
14 & 866 & 866 & 866 & 866 & 866 \\
15 & 1082 & 1082 & 1082 & 1082 & 1082 \\
\hline
\end{tabular}

Tabela 7.11: Número de becos-sem-saída detectados por cada método em 15 instâncias do dominio Drive.

\subsubsection{Detecção de becos-sem-saída no domínio Exploding Blocks World}

Na Tabela 7.7, podemos observar que o número de estados em cada instância do domínio ExplodingBlocksWorld é muito grande, assim, só foi possível avaliar 4 instâncias desse domínio (Tabela 7.12). A heurística $p d b \mid h_{m-m}$ não conseguiu avaliar nenhuma instância dentro do tempo estipulado. Observamos que o método SWR-DE e as heurísticas $h_{m-m}$ conseguiram detectar todos os becossem-saída do conjunto de estados alcançáveis. No entanto, as heurísticas $h_{1}, h_{F F}, h_{a d d}$ e $p d b$ não identificaram todos os becos-sem-saída, sendo que $p d b$ só conseguiu avaliar as 2 instâncias menores.

Como o número de estados deste domínio cresce muito rápido, chegando à ordem de milhões já na terceira instância, o tempo para computar todo o conjunto de estados $\mathcal{R}$ é muito grande, o que 


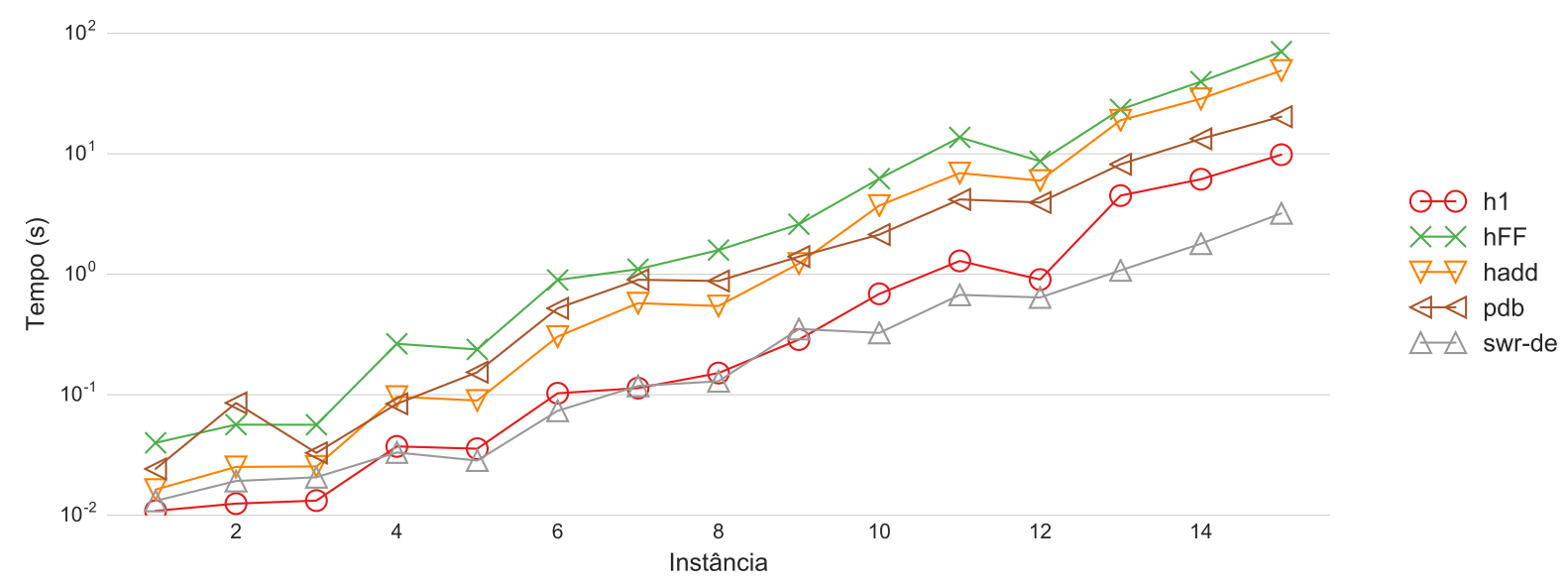

Figura 7.8: Tempo gasto para avaliar todos os estados no domínio Drive.

\begin{tabular}{|l|r|r|r|r|r|r|r|r|r|}
\hline Instância & $h_{1} \mid$ & $h_{1} \mid h_{m-m}$ & $h_{F F}$ & $h_{F F} \mid h_{m-m}$ & $h_{\text {add }}$ & $h_{a d d} \mid h_{m-m}$ & $p d b|p d b| h_{m-m} \mid$ SWR-DE \\
\hline 1 & 167741 & 168803 & 167741 & 168803 & 167741 & 168803 & 84653 & - & 168803 \\
2 & 183807 & 185970 & 183807 & 185970 & 183807 & 185970 & 120030 & - & 185970 \\
3 & 4242645 & 4252785 & 4242645 & 4252785 & 4242645 & 4252785 & - & - & 4252785 \\
4 & 4410368 & 4413586 & 4410368 & 4413586 & 4410368 & 4413586 & - & - & 4413586 \\
\hline
\end{tabular}

Tabela 7.12: Número de becos-sem-saída detectados por cada método em 4 instâncias do dominio ExplodingBlocks World.

explica o rápido crescimento do tempo consumido pelo método SWR-DE. A Figura 7.9 mostra que SWR-DE consumiu menos tempo que a heurística $h_{m-m}$, com exceção da instância 4 em que $h_{m-m}$ consumiu um tempo menor.

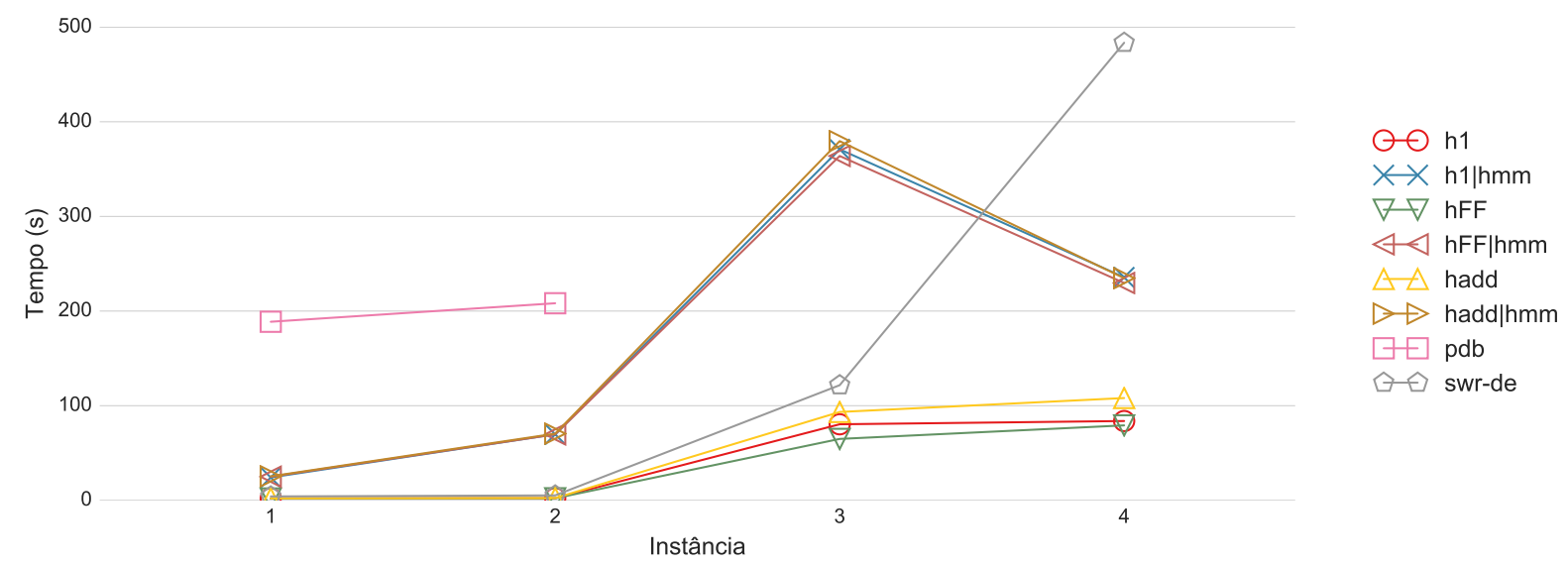

Figura 7.9: Tempo gasto para avaliar todos os estados no dominio ExplodingBlocks World.

\subsubsection{Detecção de becos-sem-saída: discussão final}

Como esperado, todas as heurísticas foram capazes de detectar becos-sem-saída em que nenhuma ação adiciona uma das proposições da meta (Navigation_1 e TriangleTireWorld_1) e aqueles em que nenhuma ação é aplicável (Navigation_o e TriangleTireWorld_0).

Notamos que existe um compromisso entre o tempo para estimar o valor inicial de cada estado $s$, isto é, $V^{0}(s)$, e a detecção completa dos becos-sem-saída. De um lado, heurísticas calculadas no espaço de átomos $\left(h_{a d d}, h_{F F}\right.$ e $\left.h_{1}\right)$, demandam um tempo menor para sua computação, porém, por assumirem a independência para alcançar um conjunto de proposições, elas falham na detecção de becos-sem-saída. Por outro lado, as heurísticas que consideram a dependência das proposições $\left(h_{2}\right.$, 
$p d b$ e $h_{m-m}$ ), apesar de serem capazes de detectar mais becos-sem-saída, apresentam um alto custo computacional.

A heurística $h_{m-m}$ detectou todos os becos-sem-saída em todos os domínios (com exceção da falha apresentada no domínio Drive). Diferentemente das demais heurísticas que computam os valores dos estados considerando literais ou conjunto de literais, a heurística $h_{m-m}$ realiza uma busca completa no problema relaxado, calculando o valor heurístico por estado, isto é, sem assumir independência das proposições atômicas que descrevem o estado. No entanto, observando o tempo gasto por essa heurística, percebemos que o custo computacional para tal precisão é muito alto.

O método proposto SWR-DE se mostrou mais eficiente que todas as heurísticas avaliadas, na grande maioria dos problemas. Em particular, na extensão Navigation_2 criada para conter becossem-saída de difícil detecção, o SWR-DE gastou tempos com 2 ordens de grandeza menores que as heurísticas que conseguiram detectar os becos-sem-saída desses problemas. Note que, com exceção do domínio Navigation_o que contém becos-sem-saída de fácil detecção, o SWR-DE apresentou os melhores tempos para todas as instâncias de todos os domínios (a menos da última instância do domínio ExplodingBlocks World).

\subsection{Eficiência do planejamento com detecção de becos-sem-saída}

Nesta seção, apresentamos uma análise de desempenho de diversos algoritmos de planejamento probabilístico, com e sem o uso do método proposto SWR-DE.

Os algoritmos avaliados são: o LRTDP [Bonet e Geffner, 2003b] (equipado com diferentes heurísticas) e o HMDPP [Keyder e Geffner, 2008] (o melhor planejador da competição de planejamento probabilístico de 2008, para domínios com becos-sem-saída). Como mencionamos anteriormente, o arcabouço mGPT já possui uma implementação desses dois planejadores. Ambos os planejadores foram adaptados para resolver $f$ SSPUDE MDPs utilizando a equação de Bellman limitada (Equação 3.7) com uma penalidade $D=1000$, assim, na inicialização de $V^{0}(s)$ (Algoritmos 6.2 e 2.2) atribuise o valor $D$ para becos-sem-saída, ao invés de $\infty$. Implementamos ainda o GPCI (Goal-Probability Cost Iteration) [Kolobov et al., 2012b; Teichteil-Königsbuch, 2012] (descrito na Seção 3.1.2 e no Apêndice A) que resolve $i$ SSPUDE MDPs, para servir como base para os experimentos. Note que o GPCI é o único planejador que não foi integrado ao SWR-DE, uma vez que esse algoritmo não usa uma heurística para inicializar a função valor.

Nos experimentos com o algoritmo LRTDP nós utilizamos as heurísticas: $h_{a d d}$ [Bonet e Geffner, 2003a] (Seção 4.1.1); $h_{1}$ [Haslum e Geffner, 2000] (Seção 4.1.3); $h_{F F}$ [Hoffmann, 2001] (Seção 4.1.2); $p d b$ [Bonet e Geffner, 2005; Edelkamp, 2001] (Seção 4.1.4); e $h_{m-m}$ [Bonet e Geffner, 2005] (Seção 4.2.1) (equipada com as demais heurísticas de planejamento). Não testamos as heurísticas $h_{2}$ e $h_{2} \mid h_{m-m}$ devido ao seu alto custo computacional. Como descrito na Seção 4.2.2, o HMDPP usa uma combinação das heurísticas self-loop determinization e $p d b$.

A Tabela 7.13 mostra como cada um dos planejadores testados lida com problemas com becossem-saída. De forma geral, três estratégias foram avaliadas durante os testes. A primeira, representada pelo GPCI, expande todo o conjunto de estados alcançáveis e evita os becos-sem-saída dando prioridade às ações que maximizam a probabilidade de alcançar um estado meta. A segunda estratégia utiliza replanejamento, computando um plano e replanejando, caso um estado inesperado seja alcançado, essa estratégia é representada pelo HMDPP. A última estratégia usa programação dinâmica assíncrona para computar o valor esperado dos estados (LRTDP), limitando o valor de cada estado para garantir que o valor de cada estado convirja para um valor finito.

Cada experimento foi repetido dez vezes, nós apresentamos a média dessas repetições, quando ao menos cinco delas terminaram dentro do tempo estipulado. Para avaliar as políticas obtidas, nós utilizamos o servidor MDPSim das competições internacionais de planejamento probabilístico (IPPC5 e IPPC6), com um tempo limite de vinte minutos e cem turnos para cada experimento. Nós analisamos o desempenho em termos de tempo total de planejamento, isto é, o tempo de turnos bem sucedidos somado ao tempo gasto para computar o conjunto $\mathcal{R}$, quando esse é o caso. 


\begin{tabular}{|l|c|c|}
\hline & Problemas SSPADE MDP & Problemas iSSPUDE MDP \\
\hline GPCI & $\begin{array}{r}\text { MAXPROB seleciona estados } \\
\text { com } P^{G}(s)=1\end{array}$ & $\begin{array}{c}\text { MAXPROB da maior preferência } \\
\text { para estados com max } P^{G}(s)\end{array}$ \\
\hline HMDPP & \multicolumn{2}{|c|}{$\begin{array}{r}\text { Replanejamento }+ \text { Relaxação self-loop para reduzir } \\
\text { a chance de alcançar um beco-sem-saída }\end{array}$} \\
\hline $\begin{array}{l}\text { LRTDP }+h_{\text {add }} \\
\text { LRTDP }+h_{1} \\
\text { LRTDP }+h_{F F} \\
\text { LRTDP }+p d b \\
\text { LRTDP }+h_{m-m}\end{array}$ & $D=1000 \geq V^{*}(s), \forall s \in G^{\pi^{*}}(s)$ & $D=1000 \geq D_{\text {limite }}, \forall s \in G^{\pi^{*}}(s)$ \\
\hline
\end{tabular}

Tabela 7.13: Como os planejadores analisados resolvem problemas com becos-sem-saída.

\subsubsection{Planejamento no domínio Navigation}

Como dissemos anteriormente, problemas nos domínios Navigation_0, Navigation_1 e Navigation_2 são do tipo iSSPUDE MDP, sendo que os dos domínios Navigation_1 e Navigation_2 contêm becos-sem-saída de difícil detecção.

As Figuras 7.10, 7.11 e 7.12 mostram o tempo de planejamento para os domínios Navigation_0, Navigation_1, e Navigation_2, respectivamente. Em cada domínio, executamos os testes em 11 instâncias com quatro linhas, variando o número de colunas entre 3 e 103. Os gráficos mostram a comparação do tempo total de planejamento dos planejadores HMDPP e LRTDP (com as diferentes heurísticas) com e sem o detector de becos-sem-saída SWR-DE.

Navigation_o. No domínio Navigation_0, como os problemas possuem apenas um beco-semsaída, todos os planejadores apresentaram um desempenho melhor sem o uso do SwR-DE (Figura 7.10). Porém, o tempo adicionado pelo SWR-DE não foi significativo para a maioria das instâncias (somente para as 2 maiores instâncias o SWR-DE acrescentou um tempo maior para alguns planejadores, por exemplo, o LRTDP $+h_{1}$, o LRTDP $+h_{1} \mid h_{m-m}$ e o LRTDP $\left.+h_{F F}\right)$. Observamos ainda, que GPCI apresentou um dos melhores resultados nesse domínio, enquanto o tempo dos planejadores que
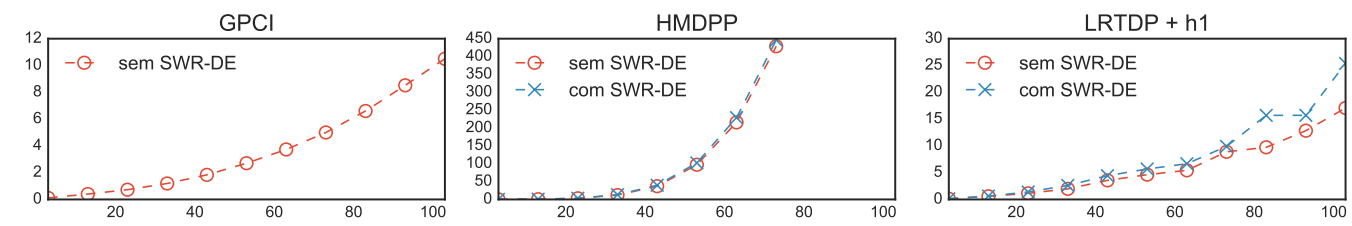

LRTDP + h1 $\mid \mathrm{hmm}$

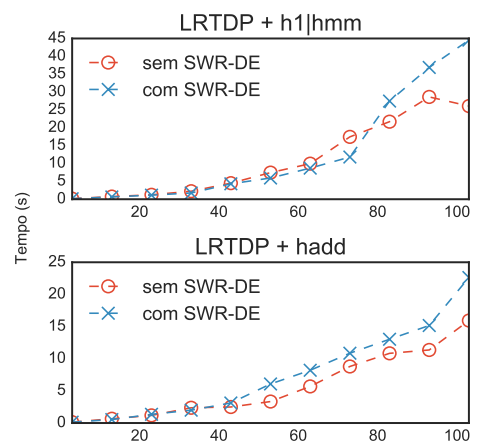

LRTDP + hFF

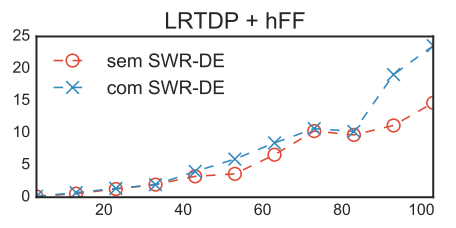

LRTDP + hFF|hmm
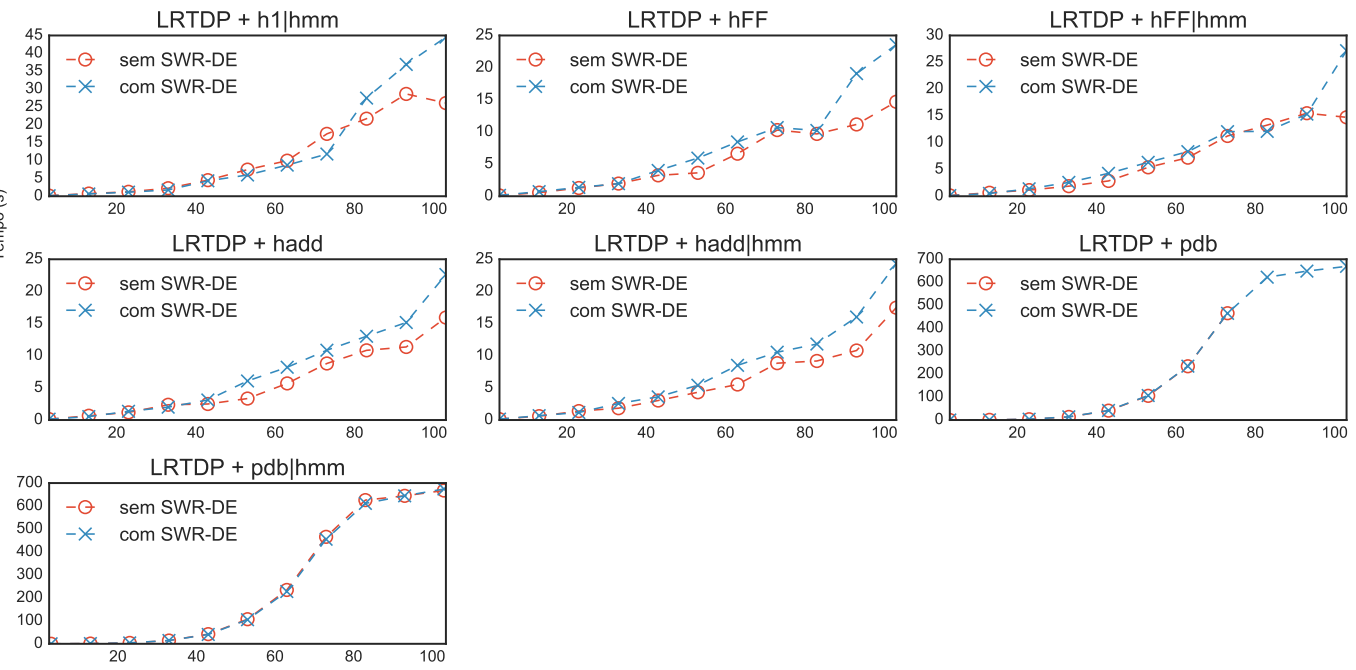

Figura 7.10: Tempo de planejamento (segundos) no domínio Navigation_0. 
utilizam a heurística $p d b$ (HMDPP, LRTDP $+p d b$ e LRTDP $+p d b \mid h_{m-m}$ ) cresceu muito rápido, chegando a ordem de centenas de segundos.

Navigation 1. No domínio Navigation_1 (Figura 7.11) apesar dos becos-sem-saída não serem explícitos, os resultados em relação ao desempenho do método SWR-DE foram semelhantes aos resultados obtidos no domínio Navigation_0. O tempo adicionado pelo SWR-DE foi um pouco mais significativo para as instâncias maiores. Note que, os planejadores que resolveram todas as instâncias mantiveram um tempo menor que 100 segundos, mesmo quando equipados com o SWR-DE.

Por outro lado, o tempo gasto pelo planejador GPCI cresceu muito e ele não conseguiu resolver a última instância dentro do tempo estipulado, um grande contraste com o bom resultado apresentado no domínio Navigation_0. Observamos ainda que, nesse domínio, os planejadores equipados com a heurística $p d b\left(\mathrm{HMDPP}, \mathrm{LRTDP}+p d b\right.$ e LRTDP $\left.+p d b \mid h_{m-m}\right)$ só conseguiram resolver até no máximo a quinta instância com 43 colunas.
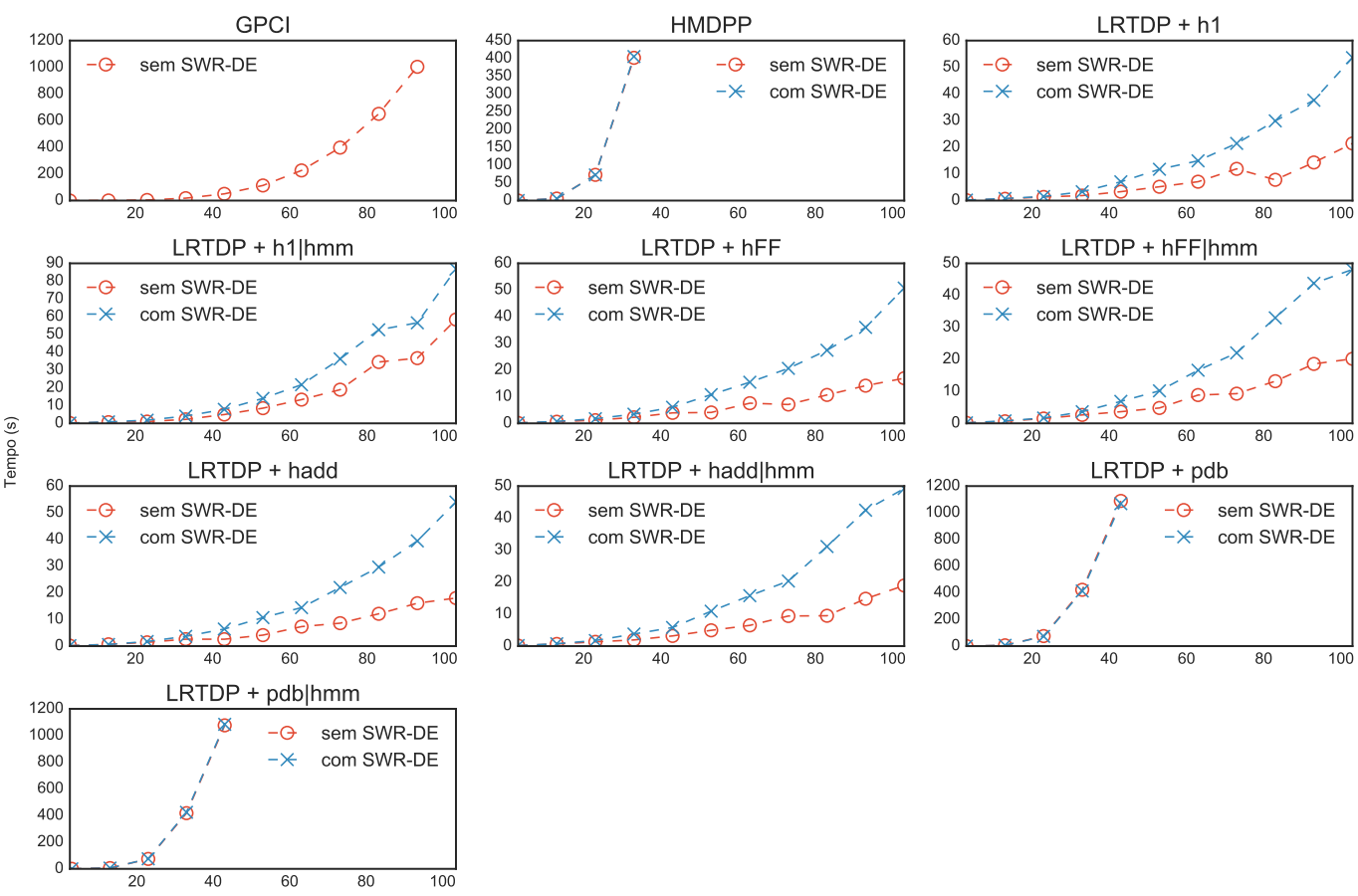

Instância

Figura 7.11: Tempo de planejamento (segundos) no domínio Navigation_1.

Navigation_2. No domínio Navigation_2 (Figura 7.12), quando equipado apenas com as heurísticas $h_{a d d}, \bar{h}_{1}, h_{F F}, h_{a d d}\left|h_{m-m}, h_{1}\right| h_{m-m}$ e $\left.h_{F F} \mid h_{m-m}\right)$ o LRTDP só resolveu as menores instâncias. Por outro lado, esses planejadores equipados com o método SWR-DE conseguiram resolver todas as instâncias. Nesse caso, conforme vimos na Seção 7.2, as heurísticas que desconsideram os efeitos negativos $\left(h_{a d d}, h_{1}\right.$ e $\left.h_{F F}\right)$ não reconhecem os becos-sem-saída, por isso o algoritmo LRTDP sem o SWR-DE, demorou muito tempo para convergir. Por outro lado, as heurísticas $h_{m-m}$ reconhecem os becos-sem-saída desse domínio, no entanto ela é muito custosa. Por isso, nesse domínio, a informação provida pelo SWR-DE aprimorou muito o desempenho do LRTDP.

Nesse domínio, o GPCI só resolveu os problemas com menos de 70 colunas. Os planejadores que usam a heurística $p d b\left(\mathrm{HMDPP}, \mathrm{LRTDP}+p d b\right.$ e LRTDP $\left.+p d b \mid h_{m-m}\right)$ só conseguiram resolver problemas com menos de trinta colunas, mesmo utilizando o método SWR-DE. Isso ocorre pois a etapa de preprocessamento da heurística $p d b$ consome muito tempo, assim esses planejadores não convergiram dentro do limite de tempo para instâncias maiores. Note que, mesmo quando equipados com o swRDE, esses algoritmos consomem a mesma quantidade de tempo, mostrando que a computação de $\mathcal{R}$ não teve um custo significativo nesses casos. 

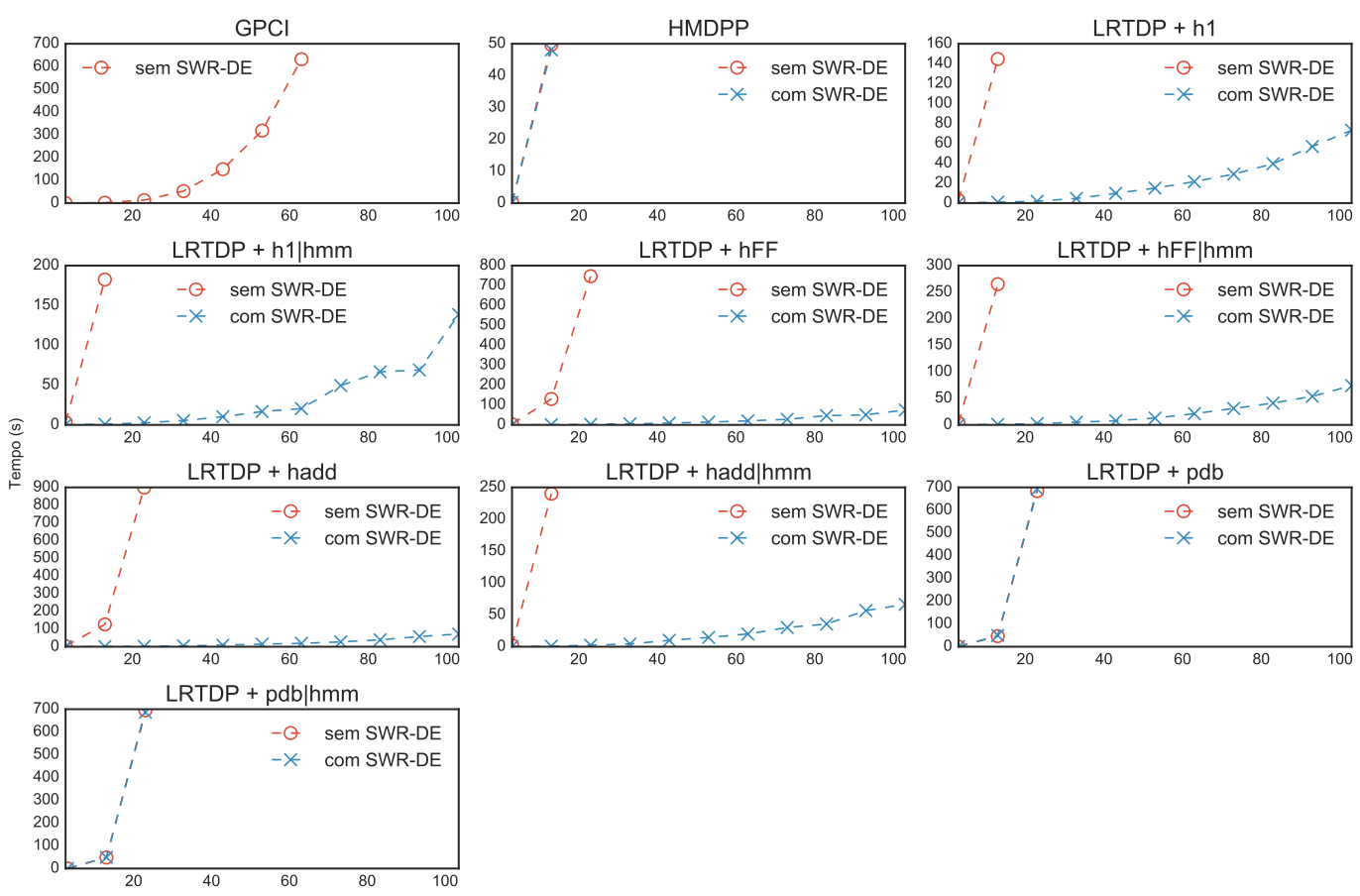

Instância

Figura 7.12: Tempo de planejamento (segundos) no dominio Navigation_2.

\subsubsection{Planejamento no domínio Triangle Tireworld}

TriangleTireworld_o Como mostramos anteriormente, os problemas do domínio TriangleTireworld_0 são SSPADE MDPs e os becos-sem-saída são facilmente detectáveis, uma vez que não há ações aplicáveis nos becos-sem-saída. A Figura 7.13 mostra o tempo de planejamento para o domínio TriangleTireworld_0. Nesse domínio, apenas o HMDPP conseguiu resolver todas as instâncias, mostrando que a heurística self-loop-determinization é muito informativa. Os demais planejadores só resolveram até a quarta ou sétima instância. Notamos que os planejadores utilizando o SWR-DE apresentaram resultados muito próximos de suas versões sem o SWR-DE, o que mostra que o tempo para computar o conjunto SWR-DE não aumentou significativamente o tempo de execução desses algoritmos. Apenas para o HMDPP, a sobrecarga do SWR-DE foi significativa.

TriangleTireworld_1 Apesar dos becos-sem-saída do domínio TriangleTireworld_1 possuírem ações aplicáveis, os resultados neste domínio foram iguais ao do domínio Triangle Tire World_o como mostra a Figura 7.14. 

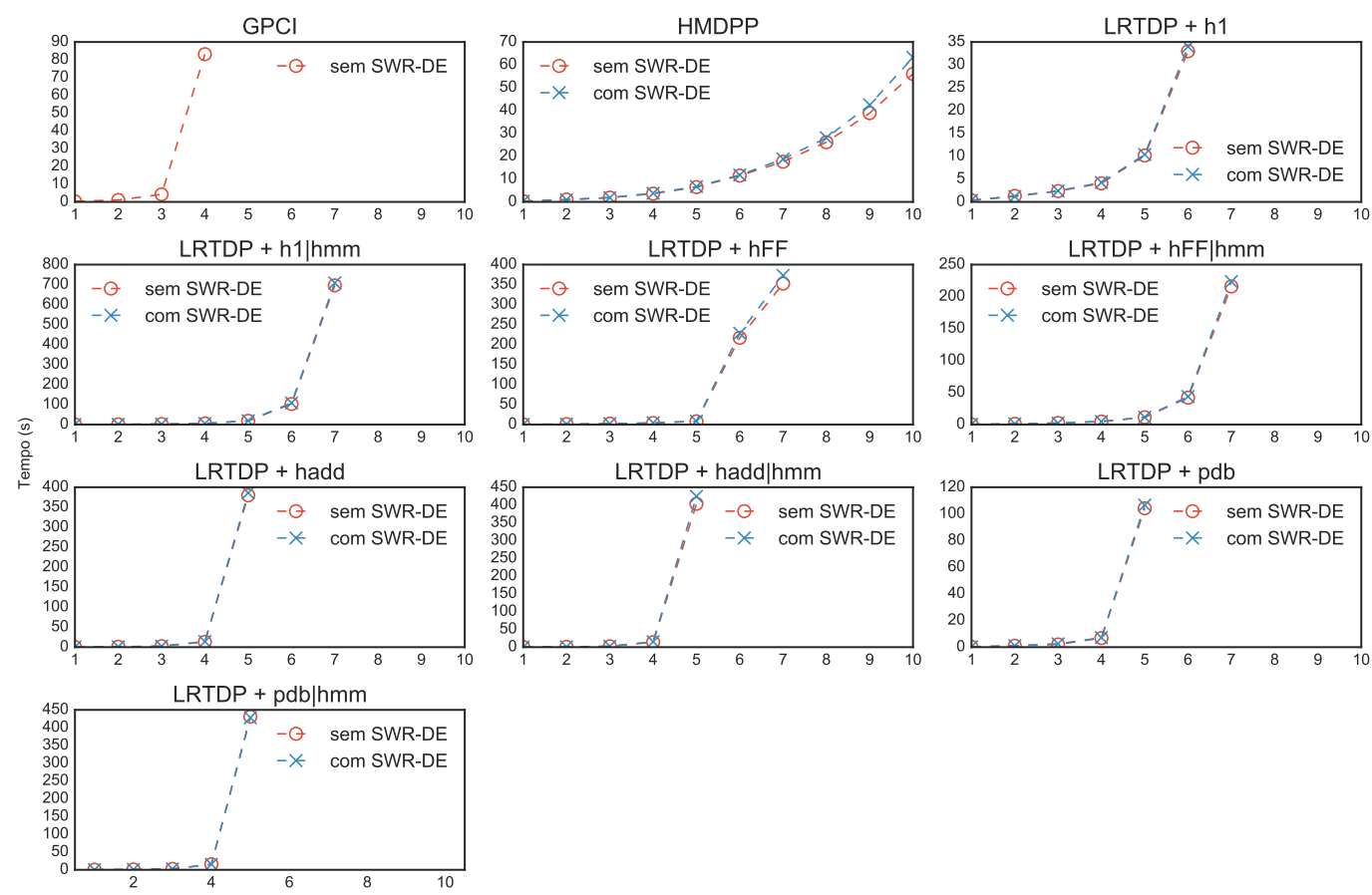

Instância

Figura 7.13: Tempo de planejamento (segundos) no dominio TriangleTireworld_o.
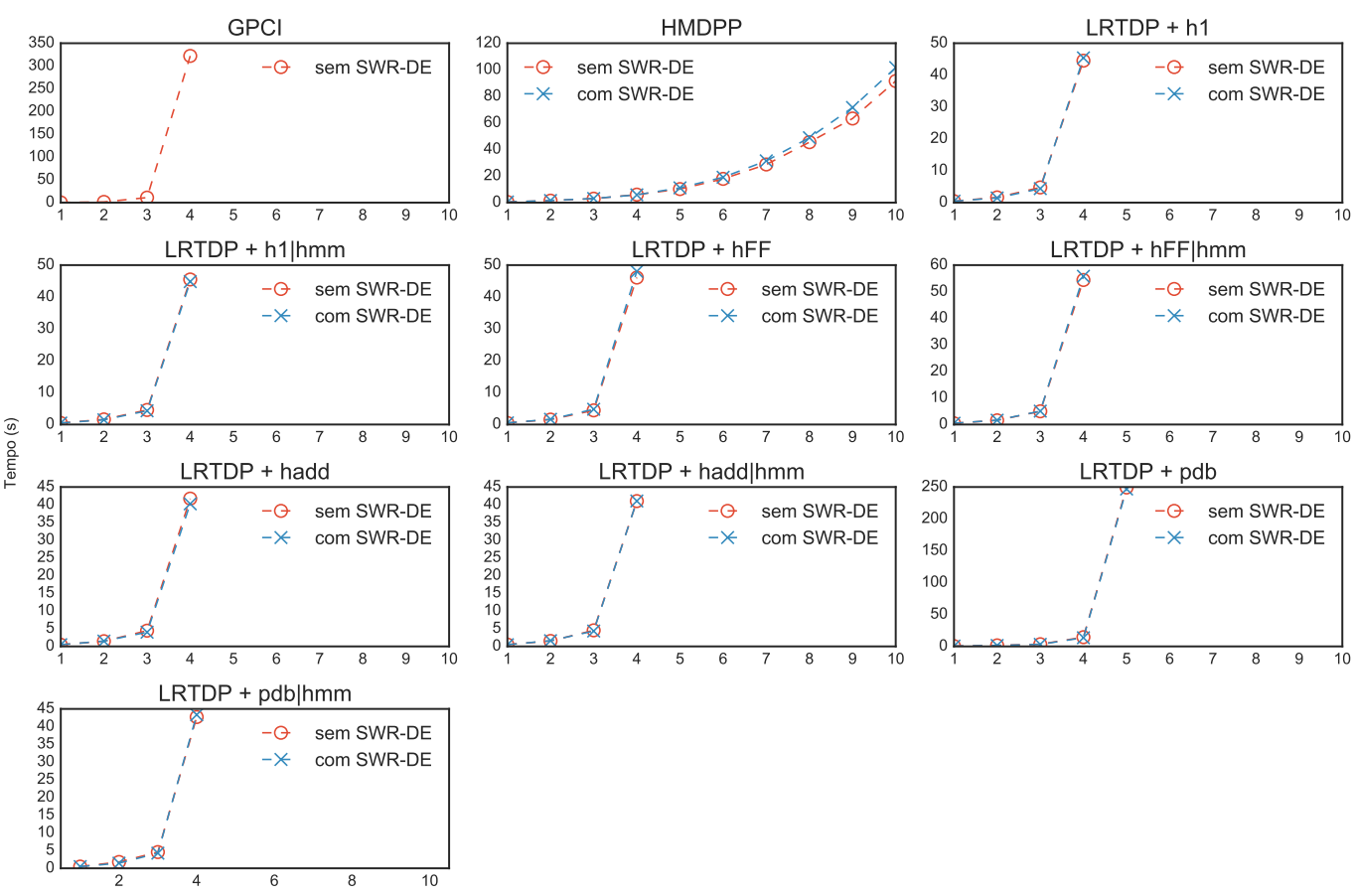

Instância

Figura 7.14: Tempo de planejamento (segundos) no dominio TriangleTireWorld_1.

\subsubsection{Planejamento no domínio Drive}

Todos os problemas do domínio Drive são iSSPUDE MDP e todos foram resolvidos em menos de 60 segundos por todos os planejadores. Os resultados deste domínio (Figura 7.15) mostram que o método de detecção de becos-sem-saída SWR-DE reduziu o tempo de planejamento do LRTDP $+h_{F F}$ na última instância. Nos demais planejadores, o uso do SWR-DE aumentou o tempo, mas apenas nas instâncias maiores. 

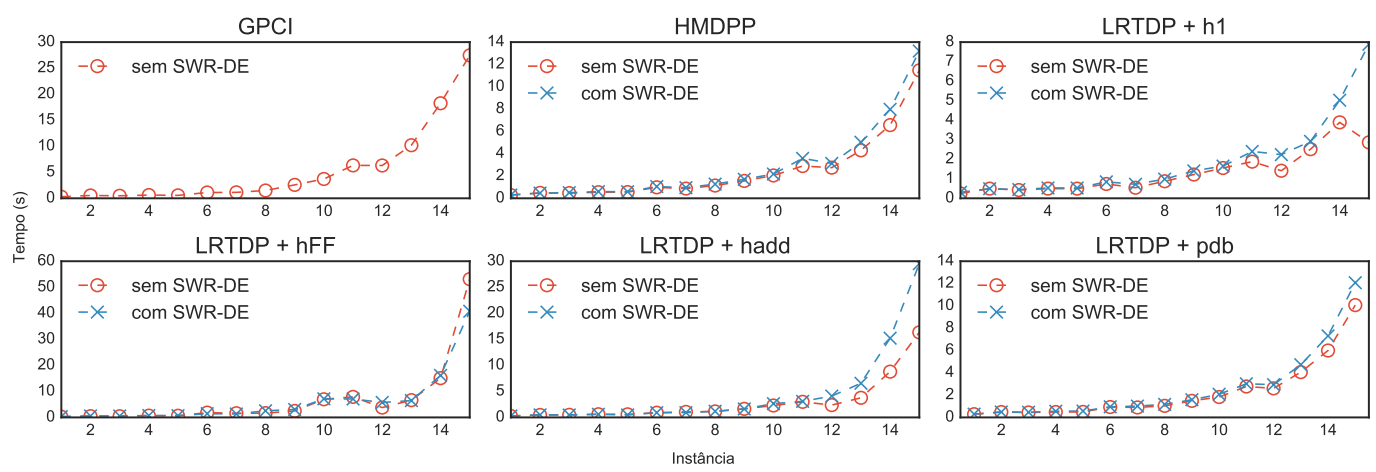

Figura 7.15: Tempo de planejamento (segundos) no domínio Drive.

\subsubsection{Planejamento no domínio Exploding Bolcks World}

Os problemas do domínio ExplodingBlocksWorld são iSSPUDE MDP e não são facilmente detectáveis. A Figura 7.16 mostra os resultados no domínio ExplodingBlocks World. Como vimos na Figura 7.9 o custo para computar $\mathcal{R}$ pode ser muito alto quando existe um conjunto muito grande de estados que podem alcançar um estado meta, assim os planejadores que usaram o método SWRDE só conseguiram resolver até a quarta instância desse domínio. Esse domínio deixa evidente um problema enfrentado pelos algoritmos baseados em regressão. Enquanto os algoritmos baseados em progressão podem perder muito tempo raciocinando sobre becos-sem-saída, algoritmos baseados em regressão podem raciocinar sobre estados que não são alcançados a partir do estado inicial.
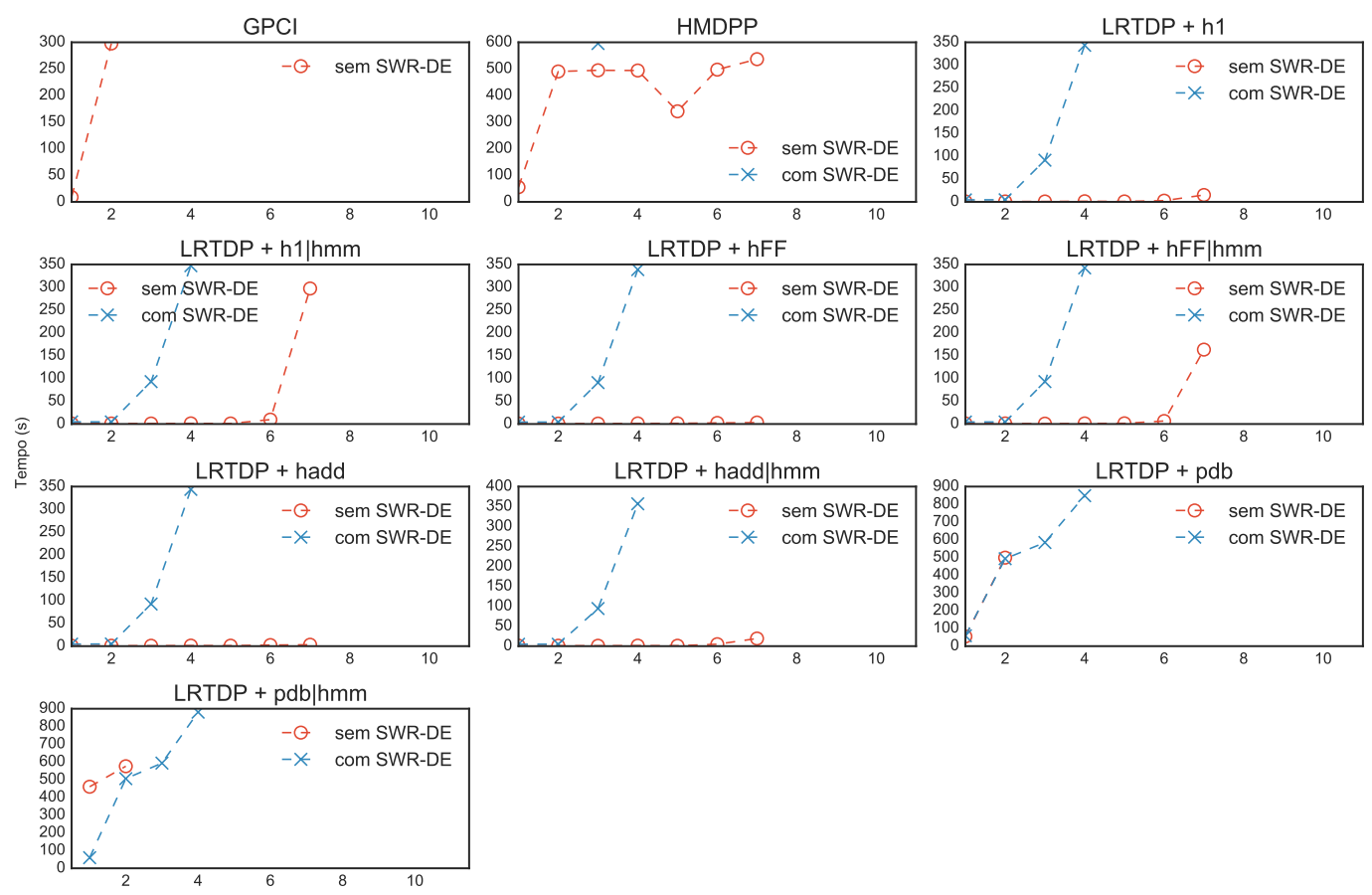

Figura 7.16: Tempo de planejamento (segundos) no dominio ExplodingBlocks World.

\subsubsection{Planejamento com becos-sem-saída: discussão final}

Observamos que a quantidade de problemas resolvidos por cada planejador depende muito das heurísticas utilizadas para inicializar a função valor, com ou sem o uso do SWR-DE.

Conforme esperado, planejadores sem o SWR-DE, demoram muito tempo para convergir quando os becos-sem-saída não são detectados pelas heurísticas. 
Nos problemas em que as heurísticas reconheceram todos os becos-sem-saída, a informação provida pelo método SWR-DE é desnecessária, mesmo assim, a sobrecarga causada pelo tempo para computar o conjunto $\mathcal{R}$ não foi significativa na maioria dos domínios (exceto no domínio ExplodingBlocks World).

Finalmente, percebemos que o método proposto, o SWR-DE, pode tornar os planejadores mais robustos, permitindo que eles resolvam problemas que não podiam ser resolvidos com heurísticas que não detectam os becos-sem-saída do problema, como no domínio Navigation_2. 


\section{Capítulo 8}

\section{Conclusão}

Neste trabalho de mestrado abordamos o problema de planejamento probabilístico na presença de becos-sem-saída. Apresentamos um estudo sobre modelos e algoritmos da área e propomos uma nova técnica de detecção de becos-sem-saída que melhora o desempenho de algoritmos considerados estado-da-arte em planejamento probabilístico.

A técnica de deteç̧ão de becos-sem-saída proposta neste trabalho é baseada na lógica temporal $\alpha$-CTL [Pereira e Barros, 2008, 2012] e operações de verificação de modelos.

Em suma, as principais contribuições desse mestrado são:

- primeira caracterização, baseada em verificação de modelos, dos diferentes tipos de problemas de planejamento probabilístico com becos-sem-saída modelados como um processo de decisão markoviano orientado à meta (GMDP): SSP MDP, SSPADE MDP e SSPUDE MDP;

- desenvolvimento de um algoritmo correto e completo para detecção de becos-sem-saída, chamado SWR-DE (detector por regressão fraca), baseado na caracterização formal com $\alpha$-CTL;

- análise experimental sobre o uso do algoritmo SWR-DE com planejadores probabilísticos; os resultados mostram que o uso do algoritmo proposto pode reduzir o tempo de convergência desses planejadores em domínios benchmark, sem adicionar um custo computacional aos planejadores considerados eficientes para problemas de planejamento probabilístico com becossem-saída.

\subsection{Trabalhos Futuros}

Conforme mostra a Seção 4.3 existem outros métodos dedicados exclusivamente para a detecção de becos-sem-saída. Seria interessante realizar uma análise comparando o método SWR-DE com esses métodos.

Em geral, métodos de detecção de becos-sem-saída também poderiam ser utilizados para fazer uma poda dos becos-sem-saída do conjunto completo de estados que serão atualizados por algoritmos de programação dinâmica síncrona, como o VI. Em trabalhos futuros pretendemos usar o método SWR-DE nos algoritmos síncronos para resolver GMDPs em que o estado inicial é desconhecido e compará-lo com outros métodos síncronos, como o TVI (Seção 2.3) e o algoritmo de iteração de política.

O método SWR-DE proposto nessa dissertação, também poderia ser utilizado por planejadores determinísticos e não-determinísticos. Nesse caso, pretendemos fazer uma análise mais extensa das heurísticas para planejamento clássico (não-determinístico) e avaliar tais planejadores com e sem o uso do SWR-DE.

É possível ainda caraterizar formalmente o diversos tipos de becos-sem-saída em problemas com observação parcial, onde o agente possui um estado de crença e nem sempre sabe exatamente em qual estado ele se encontra. Nesse caso o formalismo usado para esta caracterização deverá ser 
baseado em verificação de modelos para lógicas temporais epistêmicas [van der Meyden e Wong, 2003]. 


\section{Apêndice A}

\section{Caminho estocástico mínimo mais seguro}

Teichteil-Königsbuch [2012] e Kolobov et al. [2012b] propuseram um novo modelo para planejamento probabilístico com becos-sem-saída que ignora os caminhos que não levam para um estado meta, o $i$ SSPUDE MDP. Como vimos na Seção 3.1.2, o critério de otimização desse modelo ignora o custo de caminhos que não levam para a meta e é definido sobre dois critérios de otimização. O primeiro critério maximiza a probabilidade de atingir um estado meta e o segundo minimiza o custo esperado entre as políticas com maior probabilidade de atingir um estado meta ignorando o custo de becos-sem-saída.

O Algoritmo A.1 mostra como podemos resolver e isSPUDE MDPs. Este algoritmo faz dois processos de programação dinâmica:

1. A função IteracaoProbabilidade computa a probabilidade máxima de cada estado alcançar um estado meta de forma semelhante ao algoritmo VI. Para isso, inicializa a função de probabilidade com o valor 1 em estados meta e 0 nos demais (linhas 18 e 19). Em seguida, faz atualizações sucessivas da probabilidade de cada estado alcançar um estado meta (Linha 27 ), até que o erro residual de todos os estados seja menor que $\epsilon$.

2. A função IteracaoCusto computa o custo mínimo esperado dos históricos que levam à meta a partir de um estado $s, C^{G}(s)$ (Equação 2.11), desconsiderando históricos que não terminam em um estado meta. Para isso, ele primeiramente computa a probabilidade máxima de cada estado atingir um estado meta $P^{G}$ (Linha 2). Observe que esse algoritmo não atualiza o custo em becos-sem-saída (Linha 6). Além disso, ao atualizar o custo de um estado $C^{G}(s)$ esse algoritmo só considera as ações que maximizam $P^{G}(s)$ (Linha 10). Esse algoritmo repete a atualização da função custo $C^{G}(s)$ até que, para todos os estados, o erro residual seja menor que $\epsilon$.

Ao final, o Algoritmo A.1 devolve as duas funções que foram computadas, necessárias para encontrar a política que maximiza $J_{\pi}^{\infty}\left(s_{0}\right)$ (Equação 3.4 ). 


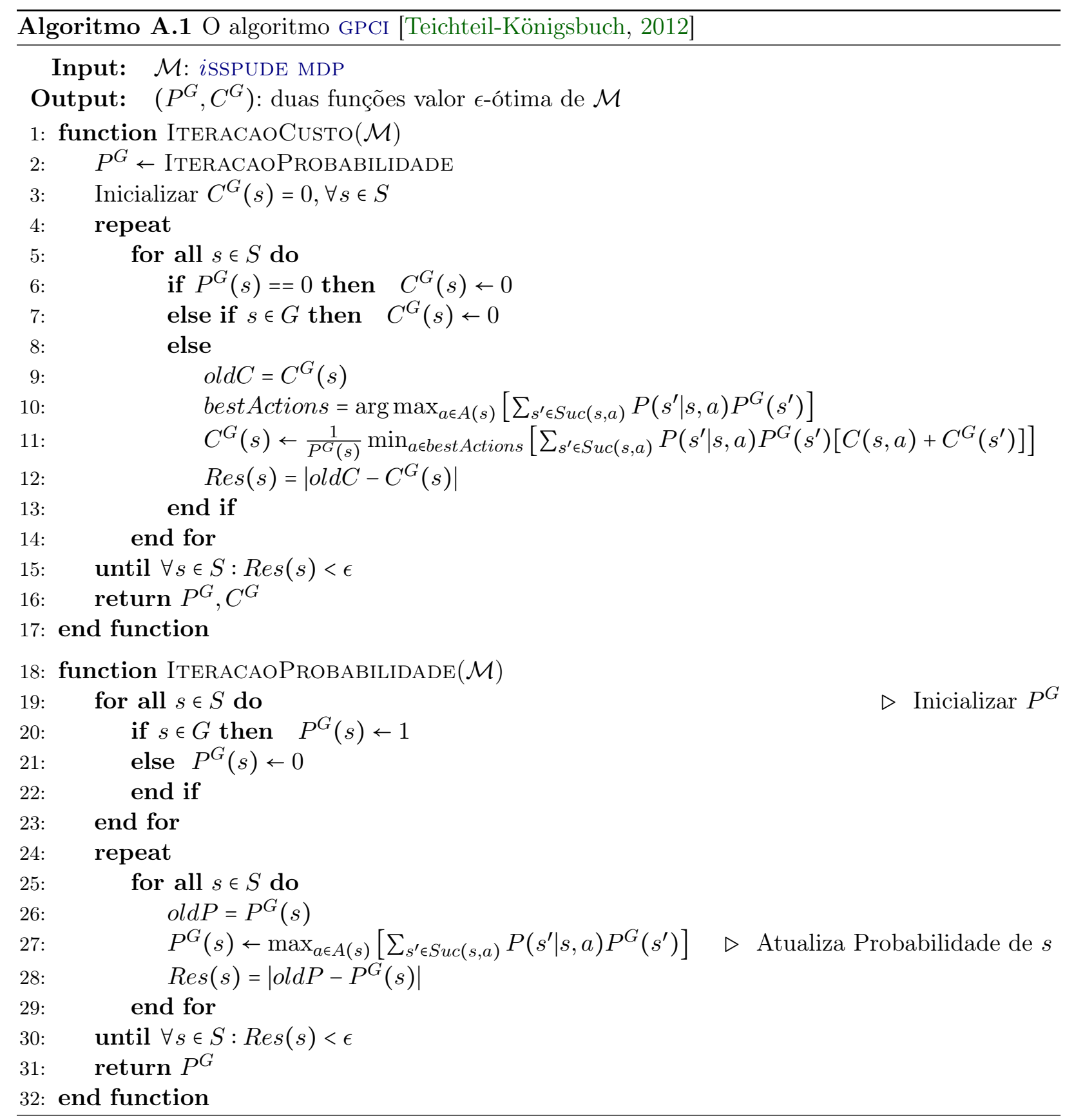




\section{Apêndice B}

\section{GMDP com desconto $\times$ SSP MDP}

Neste apêndice mostramos, para um dado SSP MDP, como a distância para a meta pode tornar políticas próprias e impróprias indistinguíveis, usando o critério de custo esperado descontado dos GMDPs com desconto.

A Figura B.1 mostra um GMDP parametrizado sem becos-sem-saída, sendo o parâmetro $n$ o número de passos entre o estado inicial $s_{0}$ e o estado meta $s_{g}$. Consideramos que o custo de todas as ações é unitário e positivo. Este problema possui apenas duas políticas $\pi_{1}=\left\{s_{0}: a_{1}, s_{1}: a_{2}, \cdots, s_{n-1}\right.$ : $\left.a_{n}\right\}$ e $\pi_{2}=\left\{s_{0}: b_{0}\right\}$. Observamos que $\pi_{1}$ é uma política própria e $\pi_{2}$ é imprópria. Como $\pi_{1}$ é própria e $J\left(\pi_{2}\right)=\infty$, o GMDP da Figura B.1 é um SSP MDP, uma vez que as duas suposições do SSP MDP são satisfeitas.

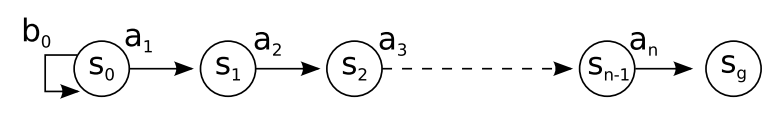

Figura B.1: Exemplo de GMDP parametrizado com duas politicas: $\pi_{1}=\left\{s_{0}: a_{1}, s_{1}: a_{2}, \cdots, s_{n-1}\right.$ : $\left.a_{n}\right\}$ e $\pi_{2}=\left\{s_{0}: b_{0}\right\}$.

Os algoritmos de programação dinâmica não distinguem duas políticas quando a diferença do valor esperado das duas é menor que o erro máximo $\epsilon$. Isto é, dadas uma função valor $J$ e duas políticas $\pi_{i}$ e $\pi_{j}$, se $\left|J\left(\pi_{i}\right)-J\left(\pi_{j}\right)\right| \leq \epsilon$ então $\pi_{i}$ e $\pi_{j}$ são $\epsilon$-indistinguíveis por $J$. Dado o GMDP parametrizado da Figura B.1, a Equação B.1 mostra que existe um $n$ para o qual as políticas $\pi_{1}$ e $\pi_{2}$ são $\epsilon$-indistinguíveis pelo critério de otimização do GMDP com desconto.

$$
\begin{aligned}
\left|J_{s_{0}}\left(\pi_{1}\right)-J_{s_{0}}\left(\pi_{2}\right)\right| & \leq \epsilon \\
\left|\sum_{t=0}^{\infty} \gamma^{t} C\left(s_{0}, b_{0}\right)-\sum_{t=0}^{n-1} \gamma^{t} C\left(s_{t}, \pi_{2}\left(s_{t}\right)\right)\right| & \leq \epsilon \\
\left|\sum_{t=0}^{\infty} \gamma^{t}-\sum_{t=0}^{n-1} \gamma^{t}\right| & \leq \epsilon \\
\left|\frac{1}{1-\gamma}-\frac{1-\gamma^{n-1}}{1-\gamma}\right| & \leq \epsilon \\
\left|\frac{\gamma^{n-1}}{1-\gamma}\right| & \leq \epsilon \\
\left|\gamma^{n-1}\right| & \leq \epsilon(1-\gamma) \\
n & \geq \log _{\gamma}(\epsilon(1-\gamma))+1
\end{aligned}
$$

Ou seja, no Exemplo da Figura B.1, quando a distância entre o estado inicial e a meta for maior que $\log _{\gamma}(\epsilon(1-\gamma))+1$, o agente não é capaz de diferenciar uma política própria de uma política imprópria. É possível generalizar esse resultado para outros exemplos.

A Figura B.2 mostra como o valor de $n$ varia quando consideramos diferentes valores para o fator de desconto $\gamma$. Fixamos o valor de $\epsilon$ em 0.001. Observamos que com um fator de desconto de 0.9, 


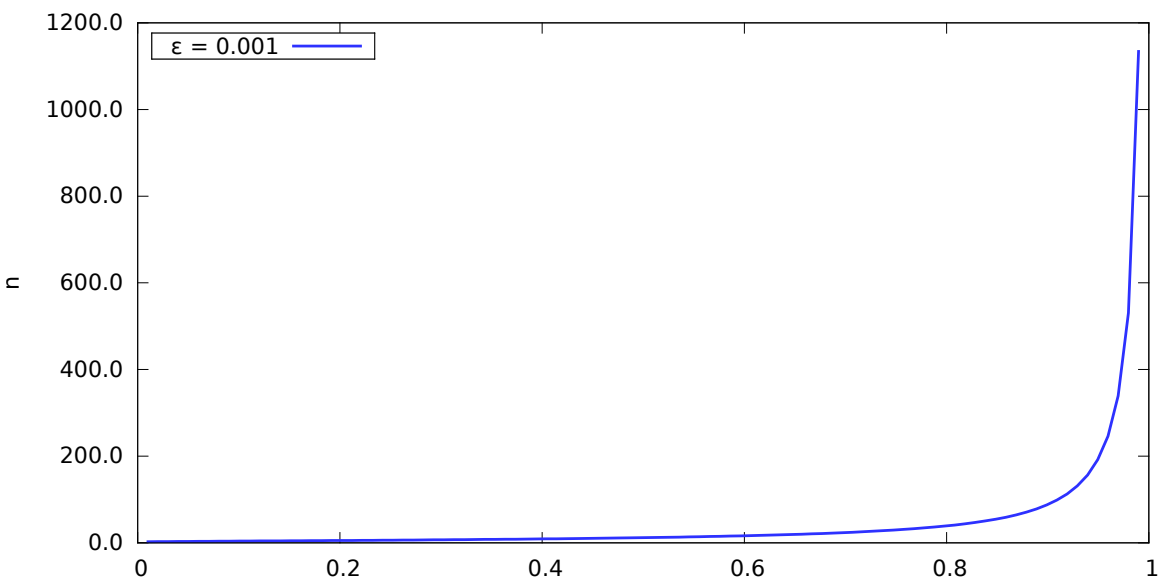

Figura B.2: Impacto do fator de desconto no GMDP da Figura B.1 no valor de n (Equação B.1)

se a meta estiver a apenas $n=89$ passos de distância do estado inicial, $\pi_{1}$ e $\pi_{2}$ são $\epsilon$-indistinguíveis por $J_{s_{0}}(\pi)$.

Em suma, para resolver um SSP MDP com o critério de otimização do GMDP com desconto seria necessário utilizar valores para $\gamma$ muito próximos de 1 . 


\section{Apêndice C}

\section{Algoritmo forte cíclico}

Neste apêndice apresentamos uma forma de encontrar o conjunto de estados a partir dos quais existe uma política forte-cíclica, isto é, os estados a partir dos quais uma política em algum momento leva o agente para um estado que satisfaz a meta.

O Algoritmo StrongCyClicPlan proposto por Cimatti et al. [2003] computa uma política forte-cíclica para um FOND $\mathcal{M}$. Primeiro esse algoritmo encontra o conjunto de estados meta $G$ (Linha 2). Esse algoritmo utiliza conjuntos de pares de estado e ação para representar o submodelo $S A$ que está sendo computado.

Primeiro, a função principal STRONGCYCLICPLAN faz um ponto fixo máximo sobre o submodelo $S A$ que inicialmente contém todas as transições (Linha 3). A cada iteração, a função remove ações que não levam para os estados de $S A$ ou para $G$ (função PRUneOUtgoig) e remove os estados em que nenhuma ação aplicável leva para $S A$ ou $G$ (função PRUNEUnCONECTED). Isso se repete até que $S A$ seja igual por duas iterações seguidas (Linha 7). Nesse ponto, o submodelo $S A$ tem subconjuntos de estados desconexos, nos quais alguma ação sempre leva para algum estado dentro do mesmo subconjunto.

Antes de devolver o resultado, o algoritmo chama a função REMOvENONPROGRESS para retirar de $S A$ os subconjuntos de estados que não estão conectados com $G$, fazendo um ponto fixo mínimo sobre o submodelo newSA que inicialmente é vazio. A cada iteração, a função REMOveNonProGRESS adiciona em new $S A$ transições que podem levar a meta computadas pela pré-imagem fraca (Linha 24). Esta operação é restrita ao submodelo $S A$ que havia sido calculado no primeiro ponto fixo, para isso, faz-se uma conjunção entre o resultado da pré-imagem e $S A$. Finalmente, o método PRUNESTATES (preimage, new $S A$ ) remove de $S A$ transições cujo estado pertence à preimage, assim, esse método só associa uma ação para cada estado. Note que dessa forma, ações que criam traps em estados que possuem outra política que podem atingir a meta também são removidas.

Assim, o conjunto $\mathcal{R}^{*}$ é o conjunto de estados do submodelo $S A$ calculado por STRONGCYCLICPlAN unido ao conjunto de estados meta. 


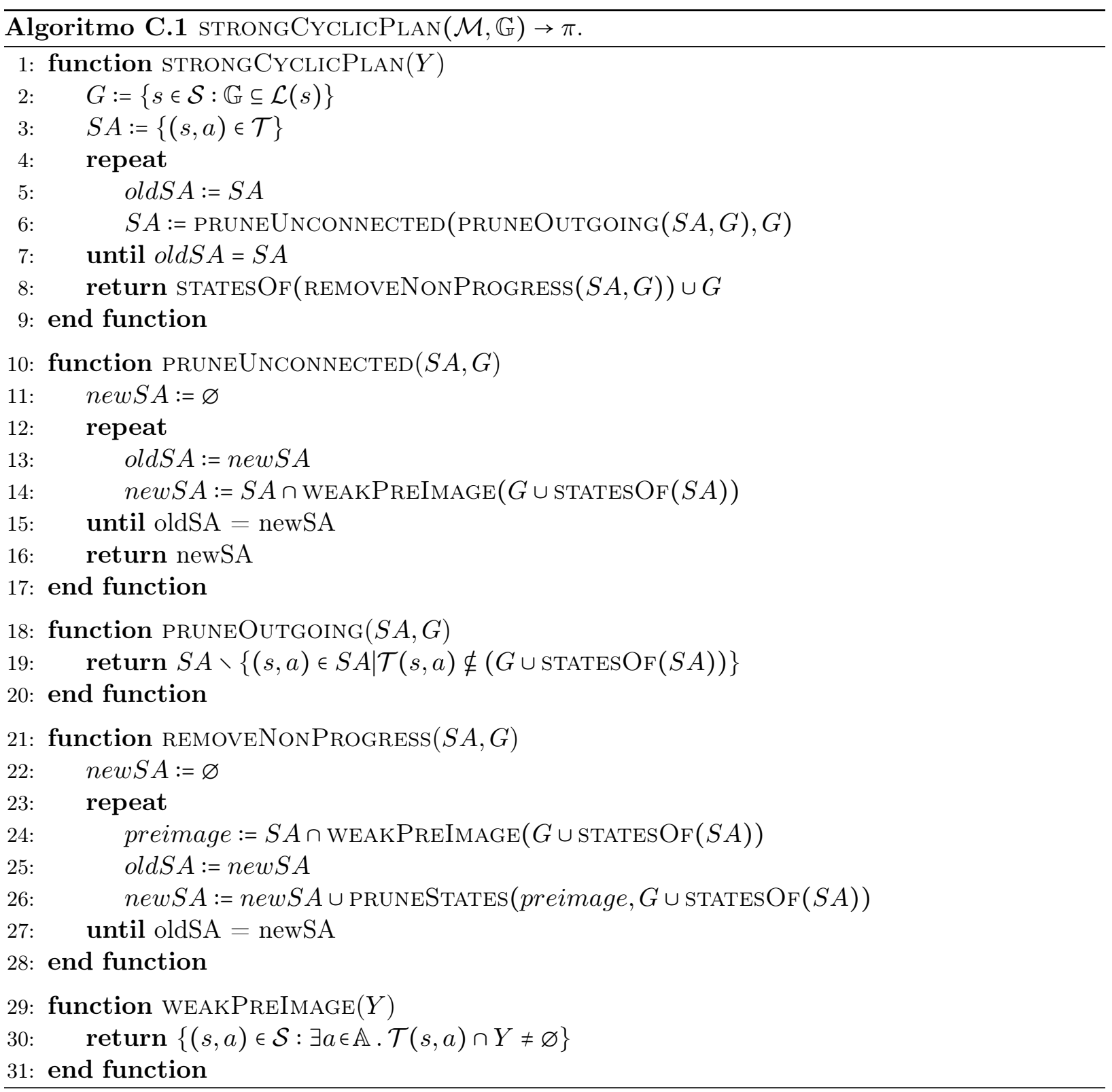




\section{Apêndice D}

\section{Especificação de domínios de planejamento probabilístico}

\section{D.1 Especificação do domínio Navigation (em RDDL)}

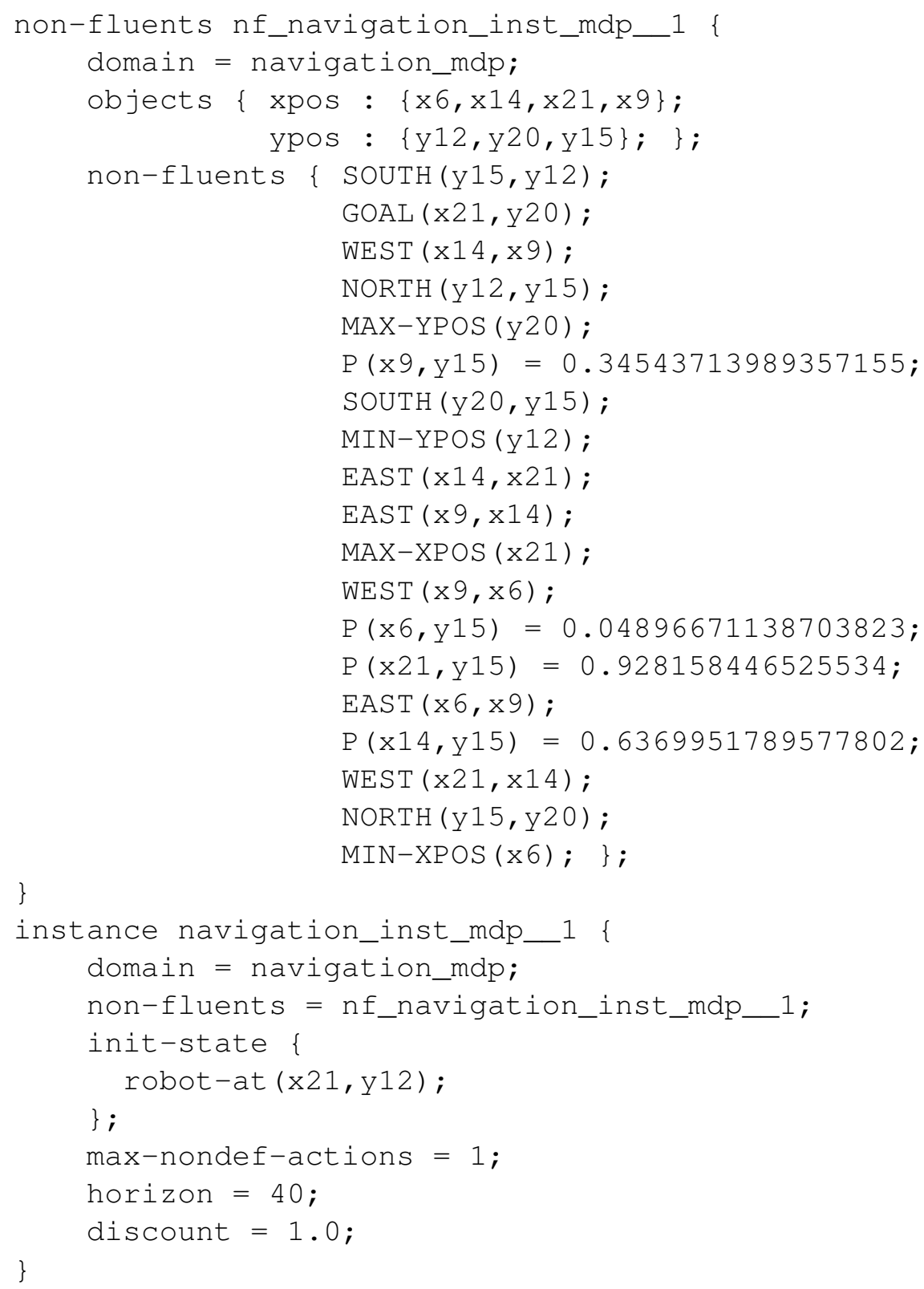




\section{D.2 Especificação do domínio Triangle Tire World (em PPDDL)}

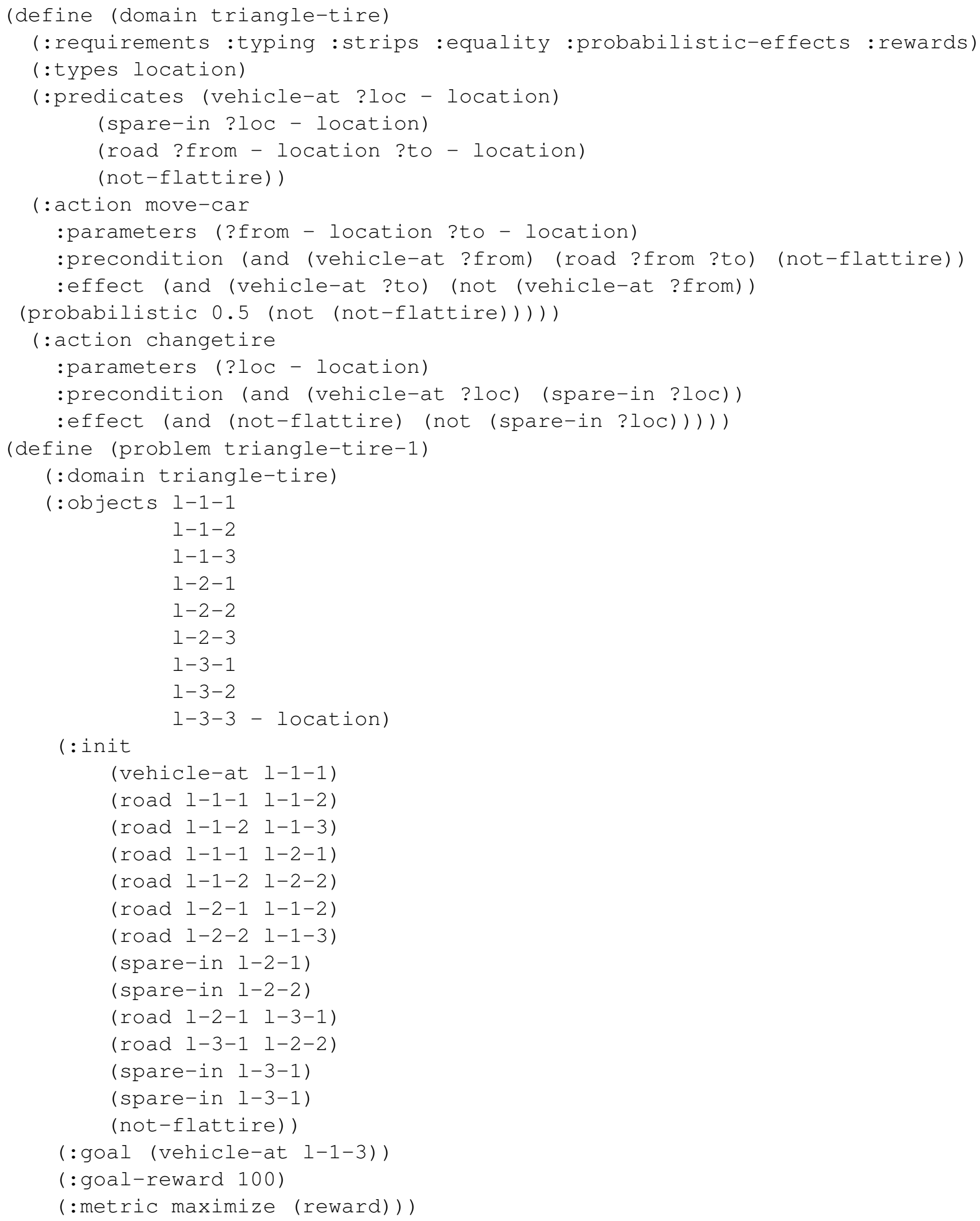




\section{Referências Bibliográficas}

Bäckström et al.(2013) Christer Bäckström, Peter Jonsson e Simon Ståhlberg. Fast detection of unsolvable planning instances using local consistency. Em SOCS 2013, Leavenworth, Washington, USA, July 11-13, 2013. Citado na pág. 36, 44, 45

Bahar et al.(1997) R Iris Bahar, Erica A Frohm, Charles M Gaona, Gary D Hachtel, Enrico Macii, Abelardo Pardo e Fabio Somenzi. Algebraic Decision Diagrams and their Applications. Formal methods in system design, 10(2-3):171-206. Citado na pág. 18

Barto et al.(1995) Andrew G Barto, Steven J Bradtke e Satinder P Singh. Learning to act using real-time dynamic programming. Artificial Intelligence, 72(1):81-138. Citado na pág. 6, 9, 16

Bellman(1957) Richard E. Bellman. Dynamic programming. Princeton University Press, Princeton, NY. Citado na pág. 4, 11

Bertsekas e Tsitsiklis(1991) Dimitri P Bertsekas e John N Tsitsiklis. An Analysis of Stochastic Shortest Path Problems. Mathematics of Operations Research, 16(3):580-595. Citado na pág. 4, 13, 15

Blum e Furst(1997) Avrim L Blum e Merrick L Furst. Fast Planning Through Planning Graph Analysis. Artificial intelligence, 90(1):281-300. Citado na pág. 3, 38

Bonet e Geffner(2001) Blai Bonet e Héctor Geffner. Planning as Heuristic Search. Artificial Intelligence, 129(1):5-33. Citado na pág. 3, 35, 37

Bonet e Geffner(2003a) Blai Bonet e Héctor Geffner. Faster Heuristic Search Algorithms for Planning with Uncertainty and Full Feedback. Em IJCAI 2003, Acapulco, Mexico, August 9-15, 2003, páginas 1233-1238. Citado na pág. 16, 74

Bonet e Geffner(2003b) Blai Bonet e Héctor Geffner. Labeled RTDP: Improving the Convergence of Real-Time Dynamic Programming. Em (ICAPS 2003), Trento, Italy, June 9-13, 2003, página 12-31. Citado na pág. xv, 4, 6, 16, 18, 74

Bonet e Geffner(2005) Blai Bonet e Héctor Geffner. mGPT: A Probabilistic Planner Based on Heuristic Search. Journal of Artificial Intelligence Research, 24:933-944. Citado na pág. 35, 36, 42, 63,74

Boutilier et al.(1999) Craig Boutilier, Thomas Dean e Steve Hanks. Decision-theoretic planning: Structural assumptions and computational leverage. Journal Of Artificial Intelligence Research, 11:1-94. Citado na pág. 18

Bryant(1986) Randal E Bryant. Graph-Based Algorithms for Boolean Function Manipulation. IEEE Transactions on computers, 100(8):677-691. Citado na pág. 18, 60

Bylander(1994) T. Bylander. The computational complexity of propositional STRIPS planning. Journal of Artificial Intelligence, 69(1-2):165-204. Citado na pág. 38 
Camacho et al.(2016) Alberto Camacho, Christian J. Muise e Sheila A. McIlraith. From FOND to Robust Probabilistic Planning: Computing Compact Policies that Bypass Avoidable Deadends. Em ICAPS 2016, London, UK, June 12-17, 2016., páginas 65-69. Citado na pág. 33

Chapman(1987) David Chapman. Planning for Conjunctive Goals. Artificial Intelligence, 32(3): 333 - 377. Citado na pág. 3

Cimatti et al.(1997) A. Cimatti, F. Giunchiglia, E. Giunchiglia e P. Traverso. Planning via Model Checking: A Decision Procedure for $\mathcal{A R}$. Em 4th European Conference on Planning (ECP-97), páginas 130-142, London, UK. Springer-Verlag. Citado na pág. 48, 50, 59

Cimatti et al.(2003) Alessandro Cimatti, Marco Pistore, Marco Roveri e Paolo Traverso. Weak, strong, and strong cyclic planning via symbolic model checking. Artificial Intelligence, 147(1-2): 35-84. Citado na pág. 48, 50, 87

Clarke e Emerson(1982) Edmund M Clarke e E Allen Emerson. Design and Synthesis of Synchronization Skeletons Using Branching-Time Temporal Logic. Em Logic of Programs, Workshop, páginas 52-71, London, UK. Springer-Verlag. Citado na pág. 49, 52

Dai e Goldsmith(2007) Peng Dai e Judy Goldsmith. Topological Value Iteration Algorithm for Markov Decision Processes. Em IJCAI Hyderabad, India, January 6-12, 2007, páginas 1860-1865. Citado na pág. 18

De Nicola e Vaandrager(1990) Rocco De Nicola e Frits W. Vaandrager. Action versus State based Logics for Transition Systems. Em Semantics of Systems of Concurrent Processes, La Roche Posay, France, 1990, páginas 407-419. Citado na pág. 51

Edelkamp(2000) Stefan Edelkamp. Heuristic Search Planning with BDDs. Em Proceedings of the 14th Workshop "New Results in Planning, Scheduling and Design"(PuK2000), Berlin, 21-22 August 2000. Citado na pág. 3

Edelkamp(2001) Stefan Edelkamp. Planning with Pattern Databases. Em ECP 2001, Toledo, Spain, September 12-14, 2001. Citado na pág. 41, 42, 74

Fikes e Nilsson(1971) Richard Fikes e Nils J. Nilsson. STRIPS: A New Approach to the Application of Theorem Proving to Problem Solving. Artificial Intelligence, 2(3/4):189-208. Citado na pág. 2,19

Fourman(2000) Michael Paul Fourman. Propositional planning. Em Proceedings of AIPS-00 Workshop on Model-Theoretic Approaches to Planning, páginas 10-17. Citado na pág. 59

Fox e Long(2003) Maria Fox e Derek Long. PDDL2.1: an extension to PDDL for expressing temporal planning domains. J. Artif. Intell. Res. (JAIR), 20:61-124. URL http://dx.doi.org/10. 1613/jair.1129. Citado na pág. 19

Geffner e Bonet(2013) Hector Geffner e Blai Bonet. A Concise Introduction to Models and Methods for Automated Planning. Synthesis Lectures on Artificial Intelligence and Machine Learning. Morgan \& Claypool Publishers. Citado na pág. xv, 16

Genesereth e Nourbakhsh(1993) M Genesereth e I Nourbakhsh. Time-Saving Tips for Problem Solving with Incomplete Iinformation. Em Proceedings of the 11th National Conference on Artificial Intelligence. Citado na pág. 48

Ghallab et al.(2004) M. Ghallab, D. Nau e P. Traverso. Automated Planning: Theory and Practice. Morgan Kaufmann Publishers Inc., San Francisco, CA, USA. Citado na pág. 1

Ghallab et al.(2016) Malik Ghallab, Dana Nau e Paolo Traverso. Automated Planning and Acting. Cambridge University Press. Citado na pág. 1, 14 
Giunchiglia e Traverso(1999) F. Giunchiglia e P. Traverso. Planning as model checking. Em European Conference on Planning, páginas 1-20. URL citeseer.ist.psu.edu/giunchiglia99planning. html. Citado na pág. 48, 50

Hansen e Zilberstein(2001) Eric A Hansen e Shlomo Zilberstein. LAO*: A heuristic search algorithm that finds solutions with loops. Artificial Intelligence, 129(1):35-62. Citado na pág. xv, $4,6,17,19$

Haslum e Geffner(2000) P Haslum e H Geffner. Admissible Heuristics for Optimal Planning. Em AI Planning Systems (AIPS 2000), páginas 140-149. Citado na pág. 39, 74

Haslum et al.(2005) Patrik Haslum, Blai Bonet e Héctor Geffner. New Admissible Heuristics for Domain-Independent Planning. Em AAAI 2005, Pittsburgh, USA, July 9-13, 2005., volume 5, páginas 9-13. Citado na pág. 42

Haslum et al.(2007) Patrik Haslum, Adi Botea, Malte Helmert, Blai Bonet e Sven Koenig. Domain-Independent Construction of Pattern Database Heuristics for Cost-Optimal Planning. Em AAAI, July 22-26, 2007, Vancouver, British Columbia, Canada, páginas 1007-1012. AAAI Press. Citado na pág. 40

Helmert et al.(2007) Malte Helmert, Patrik Haslum e Jörg Hoffmann. Flexible Abstraction Heuristics for Optimal Sequential Planning. Em ICAPS 2007, Providence, Rhode Island, USA, September 22-26, 2007, páginas 176-183. Citado na pág. 45

Hoey et al.(1999) Jesse Hoey, Robert St-Aubin, Alan J. Hu e Craig Boutilier. SPUDD: Stochastic Planning using Decision Diagrams. Em UAI 1999, páginas 279-288. Citado na pág. 18

Hoffmann(2001) Jörg Hoffmann. FF: The Fast-Forward Planning System. AI magazine, 22(3): 57. Citado na pág. $3,38,74$

Hoffmann et al.(2014) Jörg Hoffmann, Peter Kissmann e Álvaro Torralba. "Distance"? Who Cares? Tailoring Merge-and-Shrink Heuristics to Detect Unsolvability. Em ECAI 2014 - Prague, Czech Republic, páginas 441-446. Citado na pág. 36, 44, 45

Keyder e Geffner(2008) Emil Keyder e Héctor Geffner. The HMDP Planner for Planning with Probabilities. Sixth International Planning Competition at ICAPS 2008. Citado na pág. 36, 43, 74

Keyder et al.(2014) Emil Ragip Keyder, Jörg Hoffmann e Patrik Haslum. Improving Delete Relaxation Heuristics Through Explicitly Represented Conjunctions. Journal of Artificial Intelligence Research (JAIR), 50:487-533. Citado na pág. 40

Kolobov(2013) Andrey Kolobov. Scalable methods and expressive models for planning under uncertainty. Tese de Doutorado, University of Washington. Citado na pág. 29, 32

Kolobov et al.(2010) Andrey Kolobov, Mausam e Daniel S. Weld. SixthSense: Fast and Reliable Recognition of Dead Ends in MDPs. Em AAAI 2010, Atlanta, Georgia, USA, July 11-15, 2010. Citado na pág. $6,24,36,44$

Kolobov et al.(2011) Andrey Kolobov, Mausam, Daniel S. Weld e Héctor Geffner. Heuristic Search for Generalized Stochastic Shortest Path MDPs. Em ICAPS 2011, Freiburg, Germany June 11-16, 2011. Citado na pág. 5, 23, 30, 32, 33, 45

Kolobov et al.(2012a) Andrey Kolobov, Mausam e Daniel S. Weld. Discovering hidden structure in factored MDPs. Artificial Intelligence, 189:19-47. Citado na pág. 44

Kolobov et al.(2012b) Andrey Kolobov, Mausam e Daniel S. Weld. A Theory of Goal-Oriented MDPs with Dead Ends. Em UAI 2012, Catalina Island, CA, USA, páginas 438-447. Citado na pág. $5,6,23,24,25,26,27,28,29,33,44,74,83$ 
Lipovetzky et al.(2016) Nir Lipovetzky, Christian J. Muise e Hector Geffner. Traps, Invariants, and Dead-Ends. Em ICAPS 2016, London, UK., páginas 211-215. Citado na pág. 36, 44, 45

Little e Thiebaux(2007) Iain Little e Sylvie Thiebaux. Probabilistic Planning vs. Replanning. Em ICAPS Workshop on IPC: Past, Present and Future. Citado na pág. 66

Mausam e Kolobov(2012) Mausam e Andrey Kolobov. Planning with Markov Decision Processes: An AI Perspective. Morgan \& Claypool Publishers. Citado na pág. 14

Mcdermott et al.(1998) D. Mcdermott, M. Ghallab, A. Howe, C. Knoblock, A. Ram, M. Veloso, D. Weld e D. Wilkins. PDDL - The Planning Domain Definition Language. Relatório Técnico TR-98-003, Yale Center for Computational Vision and Control,. Citado na pág. 19

McMillan(1992) Kenneth Lauchlin McMillan. Symbolic Model Checking: An Approach to the State Explosion Problem. Tese de Doutorado, Carnegie Mellon University, Pittsburgh, PA, USA. Citado na pág. 59, 61

Menezes et al.(2014) Maria Viviane Menezes, Leliane Nunes Barros e Silvio Lago Pereira. Symbolic Regression for Non-Deterministic Actions. Learning 63 Nonlinear Models, 12(2):98-114. Citado na pág. 59

Menezes(2014) Maria Viviane de Menezes. Mudanças em Problemas de Planejamento sem Solução. Tese de Doutorado, Universidade de São Paulo. Citado na pág. xii, 53, 54

Muise et al.(2012) Christian J. Muise, Sheila A. McIlraith e J. Christopher Beck. Improved non-deterministic planning by exploiting state relevance. Em ICAPS 2012, Atibaia, São Paulo, Brazil, June 25-19, 2012. Citado na pág. 48

Pecheur e Raimondi(2006) Charles Pecheur e Franco Raimondi. Symbolic Model Checking of Logics with Actions. Em MoChArt 2006, páginas 1215-1222. Springer Verlag. Citado na pág. 51

Pereira(2007) Sílvio L. Pereira. Planning under Uncertainty for Extended Reachability Goals. Tese de Doutorado, Institute of Mathmatics and Statistics, University of São Paulo, São Paulo, Brazil. Citado na pág. xi, xv, 48, 50, 51, 54, 55, 56

Pereira e Barros(2008) Sílvio L. Pereira e Leliane N. Barros. A logic-based agent that plans for extended reachability goals. Autonomous Agents and Multi-Agent Systems, 16(3):327-344. Citado na pág. $48,51,52,81$

Pereira e Barros(2012) Sílvio L. Pereira e Leliane N. Barros. A planner agent that tries its best in presence of nondeterminism. Journal of Applied Logic, 10(4):419-430. Citado na pág. 48, 51, 81

Puterman(1994) Martin L. Puterman. Markov Decision Processes: Discrete Stochastic Dynamic Programming. John Wiley \& Sons, Inc., New York, NY, USA, $1^{\text {a }}$ edição. Citado na pág. 4, 9, 10, 11

Rintanen(2008) Jussi Rintanen. Regression for Classical and Nondeterministic Planning. Em ECAI, volume 178, páginas 568-572. Citado na pág. 59

Roijers et al.(2013) Diederik Marijn Roijers, Peter Vamplew, Shimon Whiteson e Richard Dazeley. A Survey of Multi-Objective Sequential Decision-Making. Journal of Artificial Intelligence Research. Citado na pág. 26

Russell e Norvig(2010) Stuart J. Russell e Peter Norvig. Artificial Intelligence - A Modern Approach. Pearson Education, $3^{\text {a }}$ edição. Citado na pág. 1, 35, 42

Sanner(2010) Scott Sanner. Relational Dynamic Influence Diagram Language (RDDL): Language Description. http://users.cecs.anu.edu.au/〜 ssanner/IPPC_2011/RDDL.pdf, 2010. Citado na pág. 19,63 
Sanner e Youn(2011) Scott Sanner e Sungwook Youn. International Probabilistic Planning Competition (IPPC), 2011. URL http://users.cecs.anu.edu.au/〜 ssanner/IPPC_2011/. Citado na pág. 63

Simão et al.(2015) Thiago Dias Simão, Leliane Nunes de Barros e Felipe Leno da Silva. Planejamento Probabilístico com Becos Sem Saída. Em XII Encontro Nacional de Inteligência Artificial (ENIAC 2015), Natal, Brasil. Citado na pág. 7

Simão et al.(2016) Thiago Dias Simão, Ignasi Andres, Viviane Bonadia dos Santos e Leliane Nunes de Barros. Heurísticas para Deteç̧ão de Becos sem Saída em Planejamento Probabilístico. Em XIII Encontro Nacional de Inteligência Artificial (ENIAC 2016), Recife, Brasil. Citado na pág. 7

Somenzi(2015) Fabio Somenzi. CUDD: CU Decision Diagram Package Release 3.0.0, 2015. Citado na pág. 63

Steinmetz e Hoffmann(2016) Marcel Steinmetz e Jörg Hoffmann. Towards Clause-Learning State Space Search: Learning to Recognize Dead-Ends. Em AAAI 2016, Phoenix, Arizona, USA., páginas 760-768. Citado na pág. 44

Steinmetz et al.(2016) Marcel Steinmetz, Jörg Hoffmann e Olivier Buffet. Revisiting Goal Probability Analysis in Probabilistic Planning. Em ICAPS 2016, London, UK, June 12-17, 2016. Citado na pág. 33, 36, 44

Teichteil-Königsbuch(2012) Florent Teichteil-Königsbuch. Stochastic Safest and Shortest Path Problems. Em AAAI. Citado na pág. xv, 5, 12, 23, 29, 74, 83, 84

Teichteil-Königsbuch et al.(2011) Florent Teichteil-Königsbuch, Vincent Vidal e Guillaume Infantes. Extending Classical Planning Heuristics to Probabilistic Planning with Dead-Ends. Em $A A A I$. Citado na pág. 5, 23, 30

Trevizan(2006) Felipe W Trevizan. Um modelo unificado para planejamento sob incerteza. Dissertação de Mestrado, Instituto de Matemática e Estatística, São Paulo. Citado na pág. 47

van der Meyden e Wong(2003) Ron van der Meyden e Ka-Shu Wong. Complete Axiomatizations for Reasoning about Knowledge and Branching time. Studia Logica, 75(1):93-123. Citado na pág. 82

Yoon et al.(2007) Sung Wook Yoon, Alan Fern e Robert Givan. FF-Replan: A Baseline for Probabilistic Planning. Em ICAPS, volume 7, páginas 352-359. Citado na pág. 36, 43

Younes e Littman(2004) Hakan L. S. Younes e Michael L Littman. PPDDL1.0: The Language for the Probabilistic Part of IPC-4. Em Proc. International Planning Competition. Citado na pág. 19 